\title{
Low-Intensity Blast-induced Mild Traumatic Brain Injury: Linking Blast Physics to Biological Outcomes
}

\author{
A Dissertation \\ presented to \\ the Faculty of the Graduate School \\ at the University of Missouri-Columbia
}

In Partial Fulfillment

of the Requirements for the Degree

Doctor of Philosophy

by

HAILONG SONG

Dr. Zezong Gu, Dissertation Supervisor

MAY 2019 
The undersigned, appointed by the dean of the Graduate School, have examined the dissertation entitled

Low-Intensity Blast-induced Mild Traumatic Brain Injury: Linking Blast Physics to Biological Outcomes

\author{
presented by Hailong Song, \\ a candidate for the degree of Doctor of Philosophy, \\ and hereby certify that, in their opinion, it is worthy of acceptance.
}

Dr. Zezong Gu

Dr. David Beversdorf

Dr. Grace Y. Sun

Dr. Shinghua Ding

Dr. Catherine E. Johnson 


\section{DEDICATION}

This work is dedicated to my parents Dr. Jinliang Song and Mrs. Xiuquan Guo, and particularly to my wife Dr. Jingwen Tan for their endless encouragement, support and love. 


\section{ACKNOWLEDGEMENTS}

I would like to express my sincere appreciation to my adviser Dr. Zezong Gu. As a neuroscientist at the forefront of translational research, he perfectly combines his passion for academic research and dedication to the understanding and treatments of human disease. With Dr. Gu as a role model in academic research, I aim to contribute to the field of biomedical research. His support and critiques, along the way of my $\mathrm{PhD}$ study, encouraged me to keep focusing on research projects, handling with difficulties, and striving for success, which made me a qualified research professional and a better person.

I also owe many thanks to Dr. Jiankun (Janice) Cui for guidance on in vivo and in vitro studies; none of the projects would have been possible and successful without your communication, participation, and management.

I am extremely grateful for the support of the members of my Advisory

Committee: Dr. Grace Y. Sun, Dr. David Beversdorf, Dr. Shinghua Ding, and Dr. Catherine E. Johnson for sharing your constructive inputs and insightful thoughts on my research projects. Particularly, I would like to thank Dr. Johnson again for the enormous efforts of setting up the experimental blast site at the Rock Mechanics and Explosives Research Center.

In addition, I would like to thank all the current and past members of the $\mathrm{Gu}$ laboratory for being great workmates and friends over the past five years. Specifically, Dr. Shanyan Chen, Dr. Zhe Qu, Dr. Dennis Chuang, Dr. Agnes Simonyi, and Dr. Landry Konan, as well as Tina Ndam, Runting Li, Delvise Fogwe, Garrett Ungerer, Maria Juarez, Luisa Alatorre, Joseph Del Rosario, and Genevieve Pentecost. 
This project would not be what it is without the support of the members of our collaborators in the 1) Department of Mining and Nuclear Engineering, Missouri University of Science and Technology: Dr. Catherine E. Johnson and Martin Langenderfer for the experimental blast set-up; 2) Department of Physics and Astronomy, Sidney Kimmel Institute for Nuclear Renaissance, University of Missouri: Dr. Graham K. Hubler for his expertise and research consulting in blast physics; 3) Office of Research and Development, Department of Veterans Affairs: Dr. Ralph G. DePalma for his expertise in trauma, enormous efforts for scientific editing, and endless advocate for this research; 4) Electron Microscopy Core Facility, University of Missouri: Dr. Tommi White and DeAna Grant for their research support and funding for using the transmission electron microscopy; 5) Department of Radiology, Stanford Univeristy: Dr. Utkan Demirci for his expert opinion in tissue engineering; 6) United States Naval Research Laboratory: Dr. David R. Mott and Dr. Doug Schwer for their expert view on blast simulation; 7) Canadian Military and Veterans' Clinical Rehabilitation, Faculty of Rehabilitation Medicine, University of Alberta: Dr. Ibolja Cernak for her experienced perspectives on trauma research; 8) Department of Neurology, University of Kansas Medical Center: Dr. Xiaowan Wang and Dr. Russell Swerdlow for their support related to mitochondria research; 9) Bedford VA Medical Center: Dr. Mei Chen and Dr. Weiming Xia for the technical support of quantitative proteomics; 10) Department of Computer Sciences, University of Missouri: Dr. Jie Hou, Chen Chen, and Dr. Jianlin Cheng for their countless support and help on computation and 'omics data analysis; 11) Department of Chemistry, Univeristy of Missouri: Dr. Mitch C. Johnson, Dr. Bo Yang, 
and Dr. Michael Greenlief for their assists on projects related to liquid chromatography and mass spectrometry.

This research work made possible with the support in part by the grants from Department of Defense Congressionally Directed Medical Research Programs (DOD CDMRP) for the Peer Reviewed Alzheimer's Research Program Convergence Science Research Award (PRARP-CSRA), Biomedical Laboratory Research and Development Service (BLR\&D) of the Veterans Affairs Office of Research and Development (VA ORD), the Cure Alzheimer's Fund, the University of Kansas Alzheimer's Disease Center Mitochondrial Genomics and Metabolism Core, The University of Missouri MIZZOU Advantage, and the Department of Pathology and Anatomical Sciences research fund.

I would like to acknowledge again for all those who have helped me greatly with other projects not mentioned in this dissertation. Particularly, I want to express my sincerely thank for 1) Center for Botanical Interaction Studies, University of Missouri: Dr. Michael Greenlief, Dr. William Folk, Dr. Jianlin Cheng, Dr. Yuan Lu, Dr. Valeri Mossine, Dr. Kevin Fritsche, and Dr. Dennis Lubahn; 2) Department of Biochemistry, University of Missouri: Dr. Thomas P. Mawhinney and Dr. Brenda Peculis; 3) Department of Chemistry and Biochemistry, University of Notre Dame: Dr. Shahriar Mobashery and Dr. Mayland Chang; 4) Department of Neurology, the Second Affiliated Clinical College of Fujian University of Traditional Chinese Medicine: Dr. Weilong Chen, Dr. Weiwu Cai, and Dr. Chenghan Wu. These research projects allowed me for being well prepared to conduct my dissertation projects, and resulted in the following publications listed in Pub Med with PMID: 27155670; 27734935; 27819064; 29963617; 30465328. 
Finally, I am indebted to my parents, close friends (Mr. Haobin Wang and Mr.

Peng Wang), and my cat (Mint) for your love, understanding, supporting and encouragement in many ways all the time. Especially to my dear wife Dr. Jingwen Tan, you are the love of my life. Your heart is full of love and affection. 


\section{TABLE OF CONTENTS}

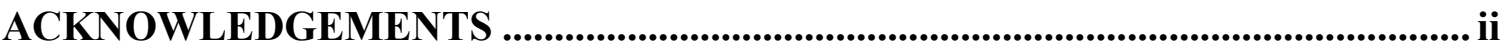

LIST OF FIGURES

LIST OF TABLES ............................................................................................................... $\mathrm{xV}$

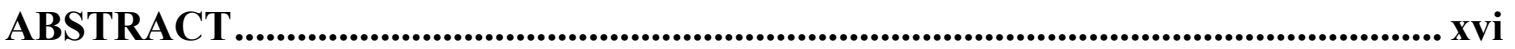

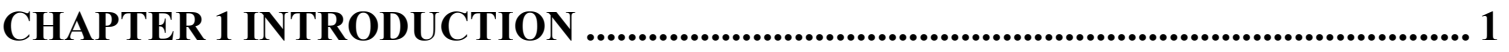

CHAPTER 2 LITERATURE REVIEW ......................................................................... 6

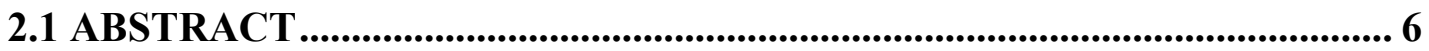

2.2 INTRODUCTION .................................................................................................. 6

2.3 PHYSICAL PRINCIPLES OF BLAST INJURIES .......................................... 8

2.3.1 Types of blast injuries....................................................................................... 9

2.3.2 Physics of explosive blast and underlying mechanisms of the blast

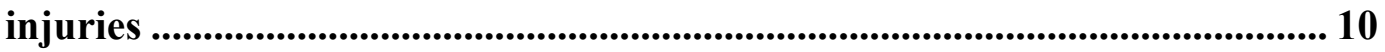

2.4 ANIMAL MODELS AND BIOLOGICAL EFFECTS OF BLAST

RELATED MTBI ON BEHAVIOR AND NEUROPATHOLOGY ..................... 14

2.4.1 Animal models of blast related mTBI ........................................................ 14

2.4.2 Behavioral changes and neuropathology of blast related mTBI ............ 23

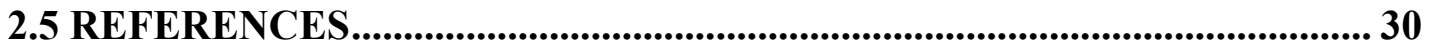

CHAPTER 3 ULTRASTRUCTURAL BRAIN ABNORMALITIES AND

ASSOCIATED BEHAVIORAL CHANGES IN MICE AFTER LOW-INTENSITY

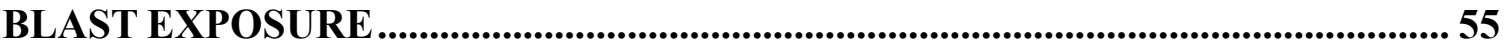

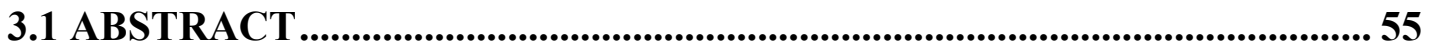

3.2 INTRODUCTION ................................................................................................. 56 


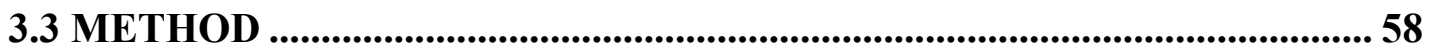

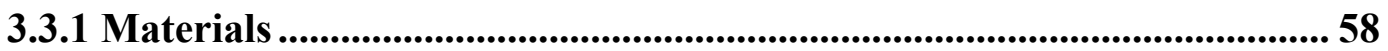

3.3.2 Experimental design and procedure ..............................................................59

3.3.3 Open-field LIB setting ...................................................................................... 60

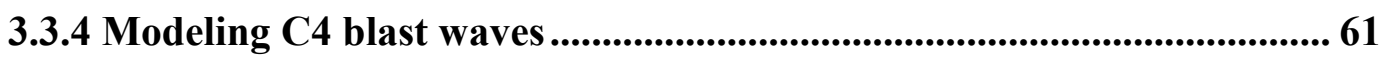

3.3.5 Tissue processing, histopathology and immunohistochemistry.............. 62

3.3.6 Transmission electron microscopy (TEM) ................................................ 65

3.3.7 Behavioral assessment ......................................................................................... 67

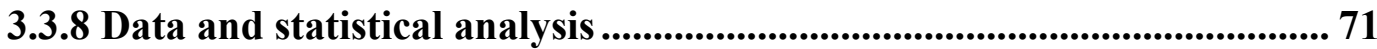

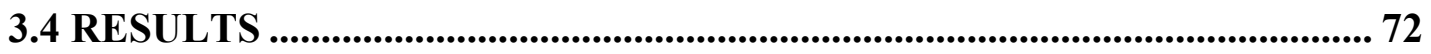

3.4.1 Characterization of LIB exposure ................................................................ 72

3.4.2 Histopathology and immunohistochemistry of blast injuries .................. 78

3.4.3 Behavioral sequelae of blast injury ............................................................... 87

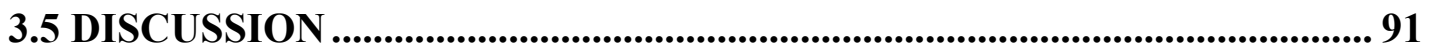

3.6 REFERENCES............................................................................................... 100

CHAPTER 4 MULTIFOCAL NEURONAL ULTRASTRUCTURAL

ABNORMALTIES AND SYNAPTIC ALTERATIONS IN MICE AFTER LOW-

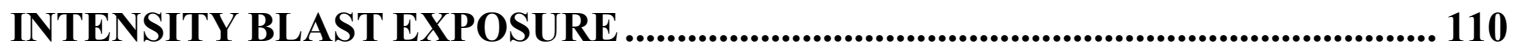

4.1 ABSTRACT ....................................................................................................... 110

4.2 INTRODUCTION ................................................................................................. 111

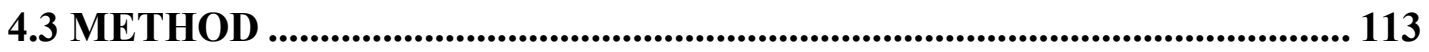

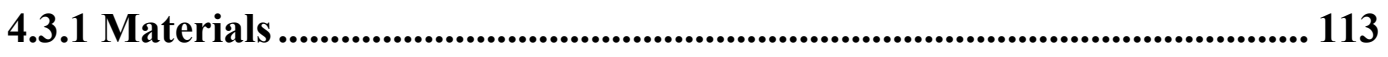

4.3.2 Animals and open-field blast setting ......................................................... 113 
4.3.3 Transmission Electron Microscopy study ............................................. 115

4.3.4 Western blot and analysis of synaptic protein ...................................... 119

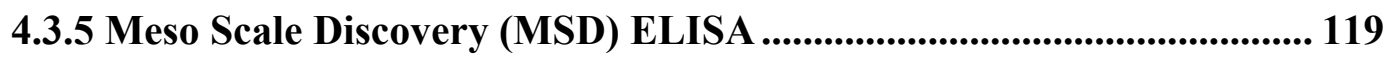

4.3.6 Statistical analyses .......................................................................................... 120

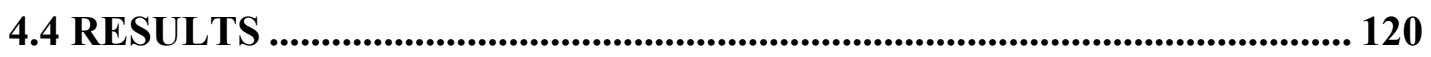

4.4.1 Degenerating neuron cell bodies and dendrites were observed after LIB

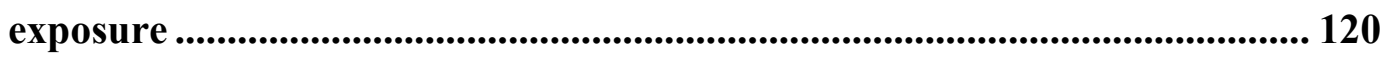

4.4.2 Microtubule abnormalities and vacuole formation in the myelinated

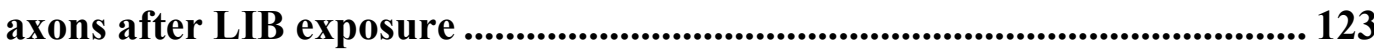

4.4.3 LIB induced axonal cytoskeletal injury associated with altered tau phosphorylation and $A \beta$ levels........................................................................... 125

4.4.4 Synaptic changes were observed after LIB exposure ............................. 126

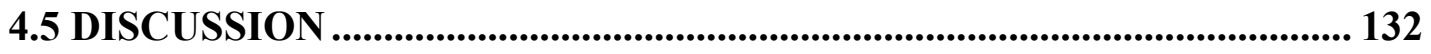

4.5.1 Blast physics underlying observed deleterious effects........................... 133

4.5.2 Changes in neuronal cell bodies ...................................................... 134

4.5.4 Cytoskeletal changes............................................................................. 134

4.5.4 Blast induced changes in the numerical density of synapses ............... 136

4.5.5 Blast caused synaptic alterations .......................................................... 138

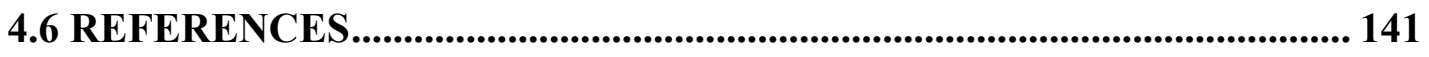

CHAPTER 5 PROTEOMIC ANALYSIS AND BIOCHEMICAL CORRELATES

OF MITOCHONDRIAL DYSFUNCTION FOLLOWING LOW-INTENSITY

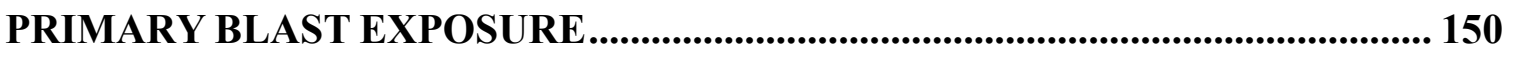

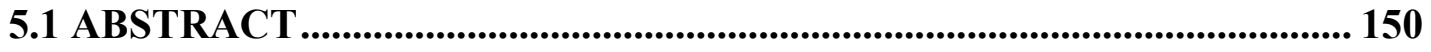


5.3.1 Materials

5.3.2 Experimental design and statistical rationale 153

5.3.3 Animals and open-field blast setting 154

5.3.4 Protein sample preparation, isobaric TMT 10-plex labeling, IMAC enrichment, LC-MS/MS analysis, and protein identification 155

5.3.5 Analysis of the mitochondrial respiratory chain 159

5.3.6 Western blot analysis 160

5.3.7 Bioinformatics and statistical analysis 161

5.4 RESULTS 162

5.4.1 Quantitative proteomic analysis of cortical global- and phosphoproteome profiles after primary LIB 162

5.4.2 GO annotation and canonical pathways associated with mitochondrial dysfunction after primary LIB 165

5.4.3 Annotation of disease and functional networks regulated after primary

LIB. 174

5.4.4 Primary LIB induced oxidative stress associated with mitochondrial dysfunction 175

5.5.5 Primary LIB altered mitochondrial dynamics, enzymatic activity on bioenergetics and compromised mitophagy 177

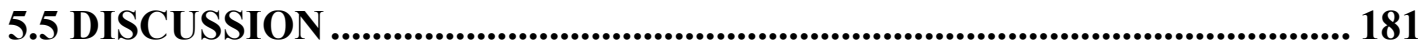

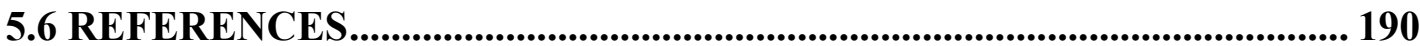




\section{CHAPTER 6 PROTEOMIC PROFILING OF MOUSE BRAINS EXPOSED TO}

BLAST-INDUCED MILD TRAUMATIC BRAIN INJURY REVEALS CHANGES

IN AXONAL PROTEINS AND PHOSPHORYLATED TAU ............................. 198

6.1 ABSTRACT .......................................................................................................... 198

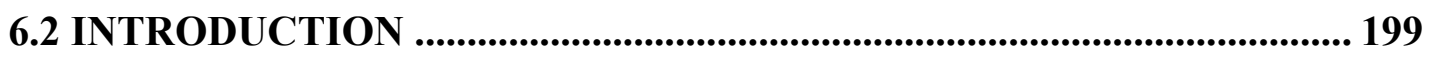

6.3 METHOD …................................................................................................... 202

6.3.1 Materials ............................................................................................. 202

6.3.2 Open-field primary blast injury in mice.................................................. 202

6.3.3 Behavioral test ............................................................................. 203

6.3.4 Brain collection and pathological examination ..................................... 203

6.3.5 Preparation of brain tissue for MS........................................................ 204

6.3.6 TMT-labeling and sample clean up.......................................................... 205

6.3.7 Phospho-protein enrichment and analysis .......................................... 205

6.3.8 LC-MS/MS analysis ......................................................................................... 206

6.3.9 Protein identification and quantification......................................... 207

6.3.10 Quantification of tau and phospho-tau by ELISA............................. 207

6.3.11 Data analysis.................................................................................................... 208

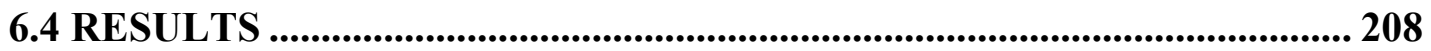

6.4.1 Increase of phospho-tau in brains of mice at 3 and 24 hours after the

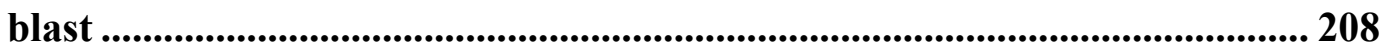

6.4.2 Behavioral and pathological alteration in mice after the blast............ 210

6.4.3 Global protein alteration in brains exposed to blast ............................. 212

6.4.4 Alteration of phospho proteomics in brains of mice exposed to blast. 217 
6.5.1 Proteomic analysis of the moue brain tissues revealed significant changes in protein expression representing axonal injury and microtubule cytoskeleton organization in response to injury ............................................... 222

6.5.2 Up-regulated calcium signaling after TBI ...................................... 226

6.5.3 Increases of p-tau, behavioral and pathological changes in mice exposed to blast link mTBI to AD ............................................................................. 228

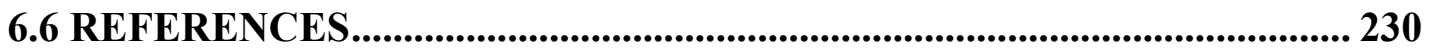

CHAPTER 7 CONCLUSIONS AND FUTURE PERSPECTIVES ....................... 240

7.1 REFERENCES.................................................................................................. 249

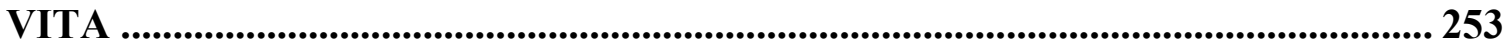




\section{LIST OF FIGURES}

Figure 1.1 The overarching hypothesis and predicted mechanism of cellular damage induced by primary LIB-induced mTBI. ................................................................ 2

Figure 2.1 Correlation of mortality rate of animals with blast peak pressure and comparison of mortality rate among different levels of blast peak pressure. ................. 24

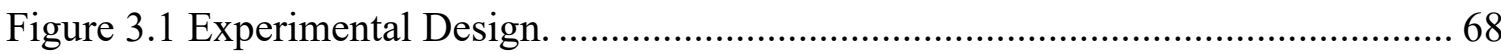

Figure 3.2 Open-field blast setting, characterization and kinetics.............................. 73

Figure 3.3 Blast overpressures, positive phase durations, and maximum impulses changed at varying blast distances.

Figure 3.4 Weather conditions of the ambient environment in the open-field blast quarry did not affect blast conditions 76

Figure 3.5 Serial images from high-speed video confirm no apparent head movement of the mice exposed to low-intensity open-field primary blast

Figure 3.6 Hematoxylin \& Eosin (H\&E) staining for brain tissue in 3-m blast and sham group.

Figure 3.7 GFAP expression levels not significantly altered after LIB exposure. 80

Figure 3.8 Axonal injury detected and quantified after LIB exposure by silver staining. 82 Figure 3.9 Myelin sheath defects identified in the corpus callosum after LIB exposure. 84 Figure 3.10 Mitochondrial abnormalities identified in the cortex, hippocampus, and striatum of the brain after LIB exposure both at 7 DPI and 30 DPI. 86

Figure 3.11 Behavioral sequelae of open-field LIB injury. 90

Figure 4.1 Neuronal cell body and dendritic abnormalities after LIB exposure. 122 
Figure 4.2 Microtubule changes and vacuole formations observed in myelinated axons after LIB exposure

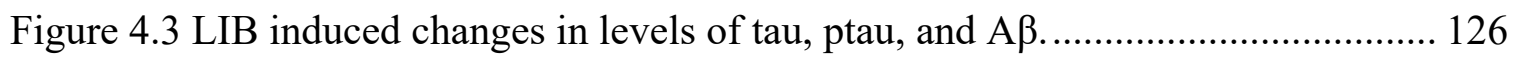

Figure 4.4 Asymmetric synaptic changes after LIB exposure.................................. 128

Figure 4.5 Decreased docked vesicles after LIB exposure...................................... 129

Figure 4.6 Quantitative assessment of the AZ length and the PSD thickness after LIB exposure.

Figure 4.7 LIB induced changes associated with pre- and post-synaptic dysregulation. 132 Figure 5.1 Proteomics analyses workflow and identification of differentially expressed global- and phospho- proteins after LIB injury. 164 Figure 5.2 Cellular components and signaling pathways enriched in global proteome at 3 hrs after LIB injury.

Figure 5.3 LIB effects at different time on alterations of canonical pathways, toxic annotation, and disease/function networks.

Figure 5.4 LIB induced mitochondrial dysfunctions associated with oxidative stress and fission-fusion dynamics. 176 Figure 5.5 LIB induced mitochondrial oxidative phosphorylation and bioenergetic failure. 178

Figure 5.6 LIB induced mitochondrial dysfunctions associated with mitophagy and autophagy. 180

Figure 5.7 A scheme depicting effects of open-field low-intensity primary blast on mitochondrial dysfunction and canonical pathways. 182 
Figure 6.1 Quantification of total tau and phosphorylated tau from mouse brain at different time points after the blast.

Figure 6.2 Neurological deficits after low intensity blast exposure assessed by SNAP test.

Figure 6.3 Axonal injury detected and quantified after LIB exposure by silver staining.

Figure $6.4 \mathrm{GO}$ ontology analysis of altered proteins at global level.

Figure 6.5 Network analysis of proteins was illustrated using the web-based tool STRING 10.0. 216

Figure 6.6 GO ontology analysis of altered brain phospho-proteomics at $3 \mathrm{~h}$ and $24 \mathrm{~h}$ post impact. 218

Figure 6.7 GO ontology analysis of altered brain phospho-proteomics at 30 days and 15 weeks post impact. 220 Figure 6.8 Network analysis of all captured phosphoproteins was illustrated using the web-based tool STRING. 


\section{LIST OF TABLES}

Table 2.1 Summary of research articles of the blast-induced behavioral outcomes........ 25

Table 2.2 Summary of the blast-induced macroscopic pathological outcomes............... 28

Table 2.3 Summary of the blast-induced neuropathological outcomes......................... 28

Table 3.1 Data for primary peak overpressure, positive phase duration and impulse of

LIB recorded from the pencil gauges mounted on each platform. …........................... 72

Table 3.2 Data of ambient environmental conditions for the LIB exposures.................. 75

Table 5.1 Canonical pathways annotation from global-proteome associated with oxidative

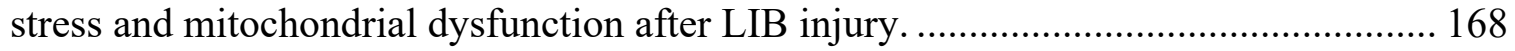

Table 5.2 Canonical pathways annotation from global-proteome associated with myelin

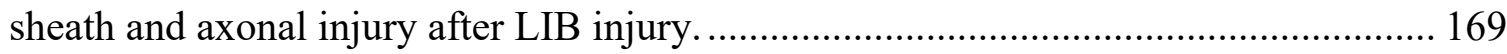

Table 5.3 Canonical pathways annotation from global-proteome associated with synaptic

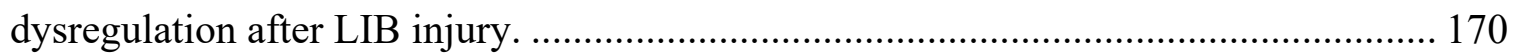




\begin{abstract}
Blast-induced mild traumatic brain injury (mTBI) is of particular concern among military personnel due to exposure to blast energy during military training and combat. The impact of primary low-intensity blast (LIB) mediated pathophysiology upon later neurobehavioral disorders has been controversial. Our prior considerations of blast physics predicted ultrastructural injuries at nanoscale levels. Here, we provide quantitative data using a LIB injury murine model exposed to open-field detonation of $350 \mathrm{~g}$ of high-energy explosive $\mathrm{C} 4$. The use of an open-field experimental blast generated a primary blast wave with a peak overpressure of 6.76 pounds per square inch (PSI) $(46.6 \mathrm{kPa})$ at a 3 -meter $(\mathrm{m})$ distance from the center of the explosion, with no apparent impact / acceleration in exposed animals. We first characterized neuropathological and behavioral changes. Using transmission electron microscopy (TEM), we further identified multifocal neuronal damages, myelin sheath defects, mitochondrial abnormalities, and synaptic dysregulation after LIB injury. Next, we used quantitative proteomics, bioinformatics analysis, biochemical investigations to seek insights into the molecular mechanisms underlying the ultrastructural pathology. Results illustrated the alterations of mitochondrial, axonal, synaptic proteins in related signaling pathways. These observations uncover unique ultrastructural brain abnormalities, biochemical correlates, and associated behavioral changes due to LIB injury. Insights on the early pathogenesis of LIB-induced brain damages provide a template for further characterization of its chronic effects, identification of potential biomarkers and targets for intervention.
\end{abstract}




\section{CHAPTER 1 INTRODUCTION}

Blast exposure leading to traumatic brain injury (TBI) is recognized as a risk for subsequent cognitive and behavioral disorders as well as chronic neurodegenerative diseases. During the conflicts in Iraq and Afghanistan, the focus on severe and penetrating brain injuries had shifted to the commonly observed mild TBI (mTBI) comprising over $82 \%$ of TBI. This type of closed head injury has been considered to be a 'signature wound' or 'invisible injury'. Low-intensity blast (LIB) injury often is salient and not detectable by conventional brain imaging techniques. Consequently, service members with blast-induced mTBI may not realize that they have sustained an injury, but later experienced post-traumatic stress symptoms (PTSD) and even neurodegenerative diseases. The pathogenesis of these injuries remains poorly characterized. The consequences of mTBI often lead to the development of progressive lifelong disabilities, imposing immense burdens on affected members, their families, and society. It is estimated that the total 10 -year cost for care, projected to 2020 , will reach up to $\$ 54$ billion for these Veterans alone. While modeling these blast injuries using shock tubes has provided valuable information on most moderate or severe brain injuries, understanding the mechanisms by which blast exposure causes mTBI remains inadequate. An urgent need exists to elucidate mechanisms underlying blast-mediated mTBI pathology and behavioral outcomes by using a reliable animal model exposed to open-field LIB that mimics combat relevant mTBI.

The objective of the dissertation is to elucidate the molecular mechanisms underlying LIB induced mTBI. Specifically, we developed an open-field LIB mouse 
model with high-energy $\mathrm{C} 4$ explosive that simulates mTBI. The overarching hypothesis is LIB injury induces mitochondrial dysfunction associated with bioenergetics impairment, which in turn induces ultrastructural damages and tau-related neuropathology leading to metabolic and behavioral deficits (Figure 1.1).
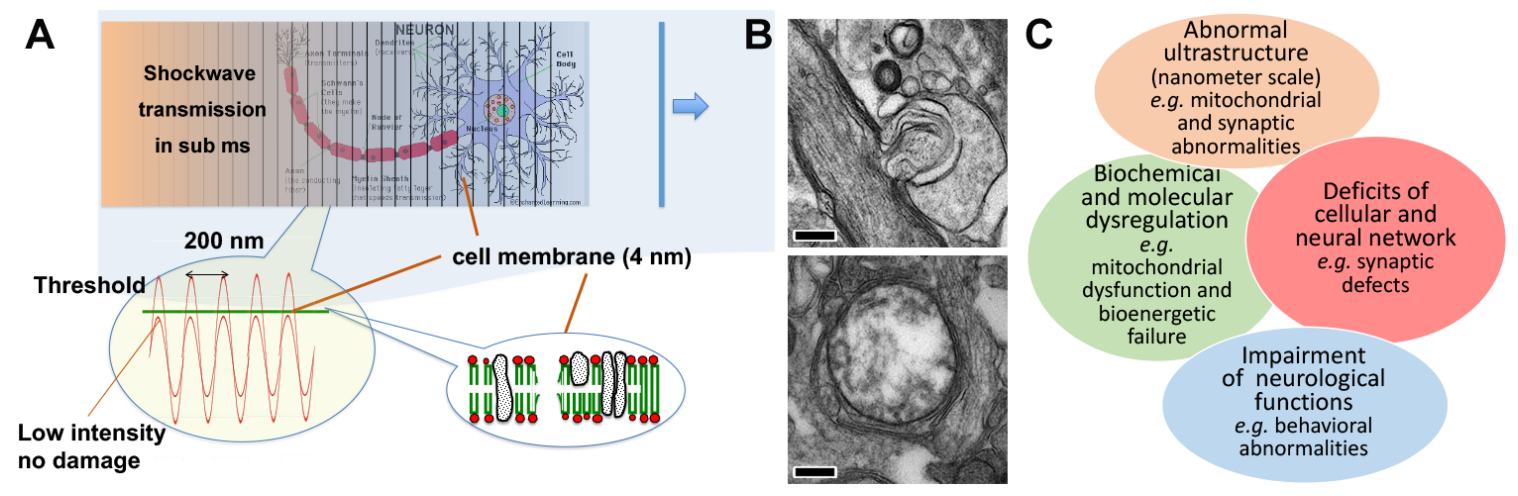

Figure 1.1 The overarching hypothesis and predicted mechanism of cellular damage induced by primary LIB-induced mTBI.

(A) The acoustic wave modeling predicts ultrastructural damage to be rupturing of tissue in the primary blast direction at intervals of approximately $200 \mathrm{~nm}$ with rupture peaks at 4 nm. (B) Representative nanoscale abnormalities, include myelin sheath ballooning (upper; scale bar: $0.5 \mu \mathrm{m}$ ) and swollen clear mitochondria (lower; scale bar: $0.2 \mu \mathrm{m}$ ). (C) Diagram of predicted biological changes, particular mitochondrial abnormalities, bioenergetics failure and synaptic defects that could lead to impairment of neurological functions. Adopted from Song et al, Neural Regen Res. 2018 with the permission.

In Chapter 2, a fundamental and comprehensive review on linking the blast physics to biological outcomes in mTBI was introduced. The role and mechanisms of primary blast wave injury remain controversial. We reviewed blast models of TBI 
including those using shock tubes and open-field blast. The behavioral and pathological findings were summarized and discussed.

In Chapter 3, we established a primary LIB injury murine model exposed to openfield detonation of $350 \mathrm{~g}$ of high-energy explosive C4, referring the Missouri Blast model. We quantified ultrastructural and behavioral changes up to 30 days post blast injury (DPI). The use of this open-field experimental blast generated a primary blast wave with a peak overpressure of 6.76 pounds per square inch (PSI) (46.6 kPa) at a 3meter (m) distance from the center of the explosion, with no apparent impact / acceleration in exposed animals. Neuropathologically, myelinated axonal damage was observed in LIB exposed groups at 7 DPI. Using transmission electron microscopy (TEM), we observed and quantified myelin sheath defects and mitochondrial abnormalities at 7 and 30 DPI. Inverse correlations between blast intensities and neurobehavioral outcomes including motor activities, anxiety levels, nesting behavior, spatial learning and memory occurred.

In Chapter 4, we studied and identified multifocal neuronal ultrastructural abnormalities and synaptic dysregulation in mice after LIB exposure. We used TEM to study the LIB effects on ultrastructural changes affecting the neuron perikaryon, axons, and synapses in the cortex and hippocampus of mice at 7 and 30 DPI. We found dysmorphic dark neuronal perikaryon and "cytoplasmic aeration" of dendritic processes, increased microtubular fragmentation of the myelinated axons, and levels of tau / phosphorylated tau (ptau) / A $\beta$. The cortical excitatory synapses decreased with a compensatory increase of the post synaptic density (PSD) thickness at 7 DPI. Additionally, we observed a significant increase of PSD95 and synaptophysin protein 
levels mainly at 7 DPI indicating synaptic reorganization. These results support the notion that a single LIB exposure leads to ultrastructural brain injury with accompanying multifocal neuronal organelle alterations.

In Chapter 5, we provided an understanding of the molecular mechanism on mitochondrial dysfunction after primary LIB exposure. The mitochondrial dysfunction includes compromised fission-fusion dynamics, diminished mitophagy, decreased oxidative phosphorylation, and compensated respiration-relevant enzyme activities that may link to neurodegeneration and cognitive impairment. Based upon these findings, we unveiled that low-intensity primary blast exposure results in the dynamic changes of global and phospho-proteomes associated with compromised mitochondrial functions. These findings provide key insights on the underlying early pathogenesis of LIB-induced mTBI for prevention and treatment.

In Chapter 6, we further revealed changes in axonal proteins and ptau using proteomic profiling. The goal of this study is to understand LIB-induced mTBI-related brain proteomic changes and tau-related biochemical adaptations that may contribute to Alzheimer's disease (AD)-like neurodegeneration. We found that both ptau and the ratio of ptau/tau were significantly increased in brains of mice collected at 3 and 24 hours (hrs) after exposure to an 82-kPa LIB injury. The results from the proteomics and bioinformatic analysis illustrated the alterations of axonal and synaptic proteins in related pathways, including but not being limited to substantia nigra degeneration, cortical cytoskeleton organization and synaptic vesicle exocytosis, suggesting a potential axonal damage caused by LIB-induced mTBI. 
Chapter 7 concludes the main findings of this study by reviewing the implications and future applications and directions of using this animal model to uncover the underlying mechanisms related to blast injury.

Overall, this work will impact our understanding on the molecular mechanisms of open-field LIB-induced mTBI. These discoveries are critical for advancing opportunities for preventive strategies and treatment of blast-induced mTBI and subsequent neurological deficits. 


\section{CHAPTER 2 LITERATURE REVIEW}

\subsection{ABSTRACT}

Blast exposures are associated with traumatic brain injury (TBI) and blast-induced TBIs are common injuries affecting military personnel. Department of Defense and Veterans Administration (DoD / VA) reports for TBI indicated that the vast majority (82.3\%) has been mild TBI (mTBI) / concussion. mTBI and associated posttraumatic stress disorders (PTSD) have been called "the invisible injury" of the current conflicts in Iraq and Afghanistan. These injuries induce varying degrees of neuropathological alterations and, in some cases, chronic cognitive, behavioral and neurological disorders. Appropriate animal models of blast-induced TBI will not only assist the understanding of physical characteristics of the blast, but also help to address the potential mechanisms. This report provides a brief overview of physical principles of blast, injury mechanisms related to blast exposure, current blast animal models, and the neurological behavioral and neuropathological findings related to blast injury in experimental settings. We describe relationships between blast peak pressures and the observed injuries. Our results indicate close relationships between blast intensities and neuropathology and behavioral deficits, particularly at low level blast intensities relevant to mTBI.

\subsection{INTRODUCTION}

Blast exposure is associated with traumatic brain injury (TBI), and also recognized as a potential risk factor for subsequent cognitive, behavioral disorders and possible chronic neurodegenerative disease [1-4]. During World War I, Frederick Mott first reported findings in post-mortem brains of three blast exposed soldiers with no physical evidence 
of external head trauma; petechial hemorrhages, mostly within the white matter of corpus callosum, and internal capsule, were noted [5, 6]. Later reports contributed to the understanding of the effects of penetrating brain injury in warfare by studying those injured in World War II and among Vietnam Veterans [7-9]. During the current conflicts in Iraq and Afghanistan [Operation Iraqi Freedom (OIF), Operation Enduring Freedom $(\mathrm{OEF})$, and Operation New Dawn (OND)], the focus on severe and penetrating brain injuries has shifted to more commonly observed closed brain injuries, particularly those referred as mild TBI (mTBI) [10]. Since 2000, Department of Defense (DoD)'s report indicated that the vast majority ( $82.3 \%)$ of TBI has been classified as $\mathrm{mTBI} /$ concussion; while only approximately $1 \%$ comprising severe injuries. Additionally, screening of one million combat veterans by the Veterans Health Administration (VHA) showed that approximately $8.4 \%$ of OEF/OIF/OND Veterans reporting and screened have received a diagnosis of TBI, most characterized as $\mathrm{mTBI}$ and, in great proportion, related to blast exposures [11, 12]. Introduction of new and improved protective body armor in 2003 led to a marked reduction of traditional combat injuries $[13,14]$. Deaths from pulmonary damage were greatly reduced by enhanced protection of the torso; however, soldiers surviving combat related blasts appeared to be at higher risk of chronic effects of mTBI caused by explosive weaponry including exposures to widely used improvised explosive devices (IEDs) [15-18]. More importantly, the pattern of neuropathology caused by blast exposure observed in the military personnel may hold unique features not observed in those who have suffered blunt TBI [18-20].

The Glasgow Coma Score (GCS) is used to evaluate TBI severity [21]. For mTBI, the GCS ranges 13 to 15 and is characterized by: a confused or disoriented state lasting 
less than 24 hours; loss of consciousness less than 30 minutes; memory loss lasting less than 24 hours, and excludes penetrating TBI $[22,23]$. In experimental models, mTBI has been found to be associated with a variety of ultrastructural, biochemical, molecular, cellular, brain circuit and behavioral abnormalities [24-28]. This type of blast induced injury, mostly from IEDs, has been described clinically as an invisible injury, except when accompanied by tympanic membrane rupture [1]. About $15 \%$ of mTBI cases chronically associate with headaches, comorbid posttraumatic stress disorders (PTSD), depression, memory disturbances, or other cognitive dysfunctions [20, 29]. It is often difficult to differentiate between the effects of blast TBI and PTSD, which may be the results of psychological stress / trauma [30-32]. Reconciling clinical reports of TBI exposure to blast in combat [33, 34], and data from experimental TBI blast models [35, 36] offers the prospects of better insights into the chronic symptoms of blast mTBI. This reconciliation may provide information about blast biomechanics and elucidate specific relationships between blast injury and later cognitive, behavior and neurological dysfunction.

We review underlying physical mechanisms of contemporary explosive devices and the spectrum of injuries inflicted by blast exposure, current shock tube and open-field mTBI animal models, and their associated behavioral and neuropathological outcomes. This narrative review is based on searches of the PubMed database using each of the terms "shock tube", "blast" or "open field blast" in combination with the terms "traumatic brain injury" or "brain trauma".

\subsection{PHYSICAL PRINCIPLES OF BLAST INJURIES}




\subsubsection{Types of blast injuries}

Most combat blast-induced mTBIs are caused by explosive weaponry such as IEDs, rocket propelled grenades, and mortars [37]. Explosive blasts can result in primary, secondary, tertiary and quaternary injuries. Primary blast injuries occur as the blast wave impacts bodily structures. Most of the shock wave impact relates to the expanding overpressure zone [38]. The primary blast wave generates internal stress and strain forces in tissues and organs to cause injury. Furthermore, depending on position in relation to blast direction and surrounding structures, subjects are also exposed to complex blast waves caused by multiple reflections from the walls, ground, and their interactions [39]. Helmets have been shown to protect the brain from lateral and posterior blast exposure [40]. Additionally, body armor protects military personnel from most ballistic projectiles to the torso, reduces lung injuries and thus increases survival [41] and, as mentioned, the likelihood of living to experience the effects of TBI.

Secondary blast injury occurs when fragments from the explosive device or ground debris impact and penetrate into the body, and is characterized as the effect of projectiles. IEDs have metal casings and are usually filled with metal fragments. Tertiary blast injury, mainly due to the blast wind, occurs when the body is thrown through space into a structure such as a building, wall or the ground. Quaternary blast injury is due to burns, asphyxia, radiation, exposure to toxic inhalants [42, 43]. Ultimately, primary injury due to blast exposure, similar to blunt trauma and ballistic penetration, relates to energy transfer from the external environment through the skull and its apertures and into the brain [44]. However, in primary blast wave injury, the modes of injuring mechanisms and coupling to tissues and structures are not immediately apparent. 


\subsubsection{Physics of explosive blast and underlying mechanisms of the blast injuries}

Here, we consider the physical properties of the blast wave, translational and rotational head acceleration mechanisms, and a novel phonon-based physical model as causes of mTBI. As mentioned, potential mechanisms for blast-induced mTBI in the military environment include direct blast wave impingement, penetrating impact, blunt impact, among others [45].

\subsubsection{Blast wave}

Modern explosives produce pressure waves along with acoustic, electromagnetic, light and thermal energies [42]. Understanding blast wave dynamics, how they interact with the human body and how the body responds is a critical step towards understanding the blast-induced mTBI.

The triggering event in an explosion is detonation. The fundamental physics of blast detonation is rapid chemical decomposition of an explosive into the shock front and blast wind $[46,47]$. When an explosion occurs, space formerly occupied by the explosive material is virtually instantaneously filled with gas under high pressure and temperature. The resulting energy expands radially outward as a blast wave moving at supersonic speed. Thereafter, the explosion may also generate nonlinear and complex blast waves depending on location of the explosive material and nearby structures [1]. The Friedlander equation $[48,49]$ characterized by a peak overpressure, duration, and impulse (integration of overpressure with respect to time) describes propagation of an ideal blast wave through time and space. A Friedlander plot depicts a very fast rise-time in positive 
pressure followed by an exponential decay to below atmospheric pressure (depending in some degree on the magnitude of the positive pressure rise) and ends upon return to atmospheric pressure $[50,51]$. The elapsed time where the blast pressure is greater than the ambient pressure (overpressure) is known as the positive-phase or impulse duration [52]. As the blast wave expands, peak shock overpressure decreases as a function of distance while its positive-phase duration increases. In high explosive blast waves, air is highly compressed on its leading edge (overpressure) creating a shock front [53]. As the shock wave expands, the density, pressure, temperature and velocity of air molecules decrease and the shocked air layer gives rise to rapidly moving air behind it, referred to as blast wind [54]. The high-pressure wave then radiates over a larger area, prolonging the duration of the over-pressurization phase. IEDs, in present conflicts, have been reported to cause a greater proportion of primary blast injuries than do conventional devices [55].

Explosive blast waves reflect off surrounding structures, including walls, buildings, vehicles and the ground. The resulting reflected pressures can be increased between two and eight times the incident pressure [54]. Reflection occurs when a single blast wave impinges on a surface, much the way an acoustic wave reflects off canyon walls creating echo. An exposed victim thus can be hit repeatedly from several directions by multiple blast waves emanating from a single explosion. Head and facial structures are more likely to be affected by direct blast wave transmission [56]. Additionally, a vascular surge from the thoracic or abdominal cavities may also transmit pressure waves into the brain $[25,57]$. Overall, blast injuries are caused by anatomical and physiological changes 
as a result of direct and reflective blast wave forces as these impact the body's surface and are transmitted internally.

\subsubsection{Translational and rotational head acceleration}

Holbourn's experimental observations using gelatin molds of brain sections showed that rotational acceleration induced widely distributed shear and tensile strains [58]. This effect may occur with long duration positive pressure phase blast waves to cause contusions and tissue shearing. A blast wave travelling at supersonic speeds can exert acceleration effects as it encounters the skull and the brain tissue [54, 57]. At maximum peak pressures, simulations show that the head might be accelerated with forces up to 300 $\mathrm{G}$ [59]. Inertial forces thus cause rapid brain motion within the skull as related to the cerebrospinal fluid (CSF) filled space between the skull and the brain to cause the brain compression and shear wave injuries [60]. Application of excess external forces also can cause the skull to collide with the brain or the brain to collide with the skull [61]. Additionally, these high impact forces may produce rotational effects causing brain injury due to stress forces, particularly at the interface between grey and white matter or between the cerebral hemispheres and the brain stem [44, 58, 62, 63]. By contrast, blast exposure at intermediate distances may not produce notable impact or accelerating effects, while higher impacts cause brain deformation [64]. Relative motion between the brain and the skull caused by head rotational-translational acceleration can result in subdural hematomas through tearing of bridging veins. Intracerebral hematomas may occur due to parenchymal blood vessel rupture during brain and skull collision $[65,66]$. 


\subsubsection{A novel phonon-based physical model for potential cause of mTBI}

Experiments have demonstrated that mTBI can also occur as a result of non-impact and low-level primary blast $[1,67]$. The effects of such low-level impacts upon brain microstructure remain unclear. The local strain and stress of the brain tissue caused by low shock wave intensities appears insufficient to cause direct tissue damage. Such damage that does occur remains invisible to conventional brain imaging $[68,69]$. The fact that mTBI fails to provide grossly observable pathological effects may be explained by a unique physical mechanistic explanation related to the length scale of the shock wave transmitted into the skull to affect brain tissue. In 2012, Kucherov et al [70] proposed a novel phonon-based biomechanical model for a potential cause of mTBI. The phonon is a quantum of energy or a quasiparticle associated with a compressional wave such as sound or a vibration of a crystal lattice. This model predicted that dimensions of brain tissue damage would occur within extremely small interval scales based upon water content of the brain and CSF: an injury range of $\sim 200 \mathrm{~nm}$ that takes place within microseconds after the blast shock wave passes through brain tissue. This hypothesis assumes that brain tissue's physical properties, on the whole, are quantitatively similar to the properties of water [71]. CSF is even closer to water in its physical characteristics. Physical considerations predict that injury can occur 1000 times more rapidly than the milliseconds (ms) characterizing classic impact and acceleration injuries. The shock wave travelling through the brain excites a phonon continuum which decays into specific acoustic waves with intensity exceeding brain tissue strength. When the intensity of the resulting acoustic oscillations exceeds the tensile strength of water, water ruptures within microseconds of the passage of the shock wave through the brain during the phonon wave 
propagation. With blast exposures, the brain may be particularly susceptible to this type of phonon-mediated injury as compared to other tissues such as muscle. The resulting damage, measured in tens to hundreds of nanometers, occurs within the period of the phonon wave propagation and therefore is difficult to detect using conventional imaging modalities. This physical analysis correlates with predictions of low intensity blast pressures well known to induce mTBI in murine experimental models $[72,73]$. This phonon-based model has also been shown to accurately describe failure waves in brittle solids [74-76]. This proposed damage mechanism requires experimental verification using ultrastructural observations to detect damage measured in the range of 4-5 nm which may occur at intervals of several hundred $\mathrm{nm}$.

\subsection{ANIMAL MODELS AND BIOLOGICAL EFFECTS OF BLAST RELATED MTBI ON BEHAVIOR AND NEUROPATHOLOGY}

\subsubsection{Animal models of blast related mTBI}

Experimental animal models have been used to address the fundamental questions related to blast-induced mTBI. Experimental approaches to examine the impact of blast waves on the brain and the consequences to mTBI require observations in live animals. Crucial questions related to blast-induced mTBI, such as physical properties and injury mechanisms can be delineated by use of reproducible experimental models. However, universal agreement between the behavioral and pathological findings observed in different animal models to military mTBI has yet to be achieved. This may due in part to the difficulty of producing primary shock waves, which maintain the characteristics of those caused by battle field blast exposures. Importantly, close proximity blast injuries 
are caused by high intensity blast waves due to the kinetic energy of the explosive process [77]. A large number of experimental settings have been used to simulate the blast injuries to study mTBI. The common forms include shock tube testing (denotation and compressed gas driven) and open-field blast settings [25, 78-81]. Kobeissy et al. provides a list of recent experimental reports on primary blast injury [82]. Among these, only 8 of total 49 reports used explosive testing to model primary blast injury. The remaining 36 studies used shock tubes configurations and amongst those, 33 used compressed gas driven tubes. Collection of repeatable blast overpressure time data, including waveform, peak overpressure, duration, impulse, pressure transducer orientation, and post-shock wind, has been stressed as vital to produce highly consistent blast injuries for reproducible research results [83]. Accurate and reproducible data are also critical in translating and scaling animal findings into human injuries [35, 84]. It remains essential to consider the potential for exposure scaling in the design of animal injury models; experimental scenarios should represent real-world exposures in so far as possible. We will later describe preliminary use of measured open-field blasts in a well characterized murine model.

\subsubsection{Shock tube settings}

Shock tubes are used in laboratory environments to produce a wide variety of repeatable blast waveforms which aim to model open-field blast waves [85]. Furthermore, shock tube testing is more economical and safe as compared to open-field blast settings [86]. However, the inherent disadvantages for shock tube experiments include the difficulties in generating shock waves approximating those due to battle field blasts, including 
reproduction of thermal and other real blast effects. Shock tube configurations tend to yield various patterns of shock waves following the initial shockwave front, which are dictated from the design of the shock tubes including shape and size. These shockwave patterns include changes in reflected shock waves, a Mach stem, an unsteady turbulent jet, and rarefaction waves, in addition to other issues on animal positioning and shock tube blockage (see below for further description) [87, 88]. Complex shockwaves within tubes can cause sudden compression or rarefaction effects upon objects in their motion path, thus transferring unusual high kinetic energy effects to animals within their path.

Shock tubes can be separated into either compressed gas- or denotation blastdriven configurations.

A compressed gas-driven shock tube is consist of horizontally mounted long circular or square steel tube used to simulate a dynamic shock wave, which was reported initially in 1955 [89]. These devices use a diaphragm, such as Mylar polyester membrane, to separate the device into two sections: a driven gas section at low pressure and a driver gas section at high pressure [90]. Shock tube diameter is determined by specimen size, with larger diameter to avoid confinement and blocking issues. Depending on the specimen dimensions, the tube diameter should be such that less than 5\% blockage of the shock wave occurs [91]. After sudden rupture or removal of the diaphragm at predetermined pressure thresholds, the pressure differential between the driver and driven gas generates shock waves which are propagated down the low pressure section (driven) towards the test object [92].

Two different driven section shock tube configurations are used to model biological blast effects. Close-ended shock tubes are capped at the end of the driven 
section. This design produces stronger and more complex reflected shock waves back into the shock tube when the shock front reaches the end of the driven section [92]. Although, use of a close-ended configuration ensures that the incident shock wave presents as one-dimensional plane wave, the inability to dissipate the gases at the end of the shock tube also induces multiple reflections that propagate through the length of the tube. Therefore, the test object is affected by repeated complex shock waveforms as compared to the original shock front [88]. In contrast, open-ended shock tubes are not capped; this configuration is open to ambient air. The result is formation of a nearly spherical shock wave when the initial shockwave front reaches the open end of the tube and an expansion wave which propagates back into the shock tube [90]. For open-ended tube configurations, two different types use either inside or outside animal placement. Inside tube placement of the animal ensures the blast wave is a planar blast wave, but causes issues related to high pressure gas confinement leading to excessive applied impulse. To avoid excessive applied impulse, the inside tube diameter must be large enough, or the specimen must be placed quite close to the end of the tube. For the outside tube configurations, the test object must be placed close to the end of the shock tube to reduce expansion wave effects which complicate planar blast wave exposure [78].

Cernak and colleagues first described the ultrastructural and functional characteristics of blast injury in rats using a large-scale (39-m long with 1-m inner diameter, maximal overpressure $291 \mathrm{kPa}$ with duration of $33 \mathrm{~ms}$ shock tube to expose the whole body. A small-scale shock tube $(0.5-\mathrm{m}$ long with inner diameter of $10 \mathrm{~mm}$, overpressure peak value $30-477 \mathrm{kPa}$ ) was used to expose localized pulmonary blast [93, 94]. In 2011, Cernak et al. further developed a helium-compressed multi-chamber shock 
tube capable of reproducing complex shock wave signatures seen in theater and tailoring pressure wave signatures [36]. Along with this shock tube design, other variants were developed to mimic blast TBI, notably including the Walter Reed Army Institute of Research (WRAIR) shock tube setting [95]. Recently, advanced blast simulator with addition of an end wave eliminator and side-vented attenuator/diffuser attached to the shock tube have been developed to enable high fidelity and more accurate simulation of open-field blast waves [96,97]. Even with these ideal compressed gas driven shock tube settings, the resultant shockwaves can differ from the open-field blasts, particularly the duration and negative phases of the blasts. Simulation using a semi-analytical approach to predict the characteristics of pseudo blast waves that form in a compressed gas driven shock tube from test configuration parameters, along with other reports, indicate that if the shock tube parameters are not designed 'correctly', the resulting shock waves have a tendency to adopt trapezoidal shapes where the peak pressure plateaus before these decay as open-field blast waves do [81, 98]. Identifying the initial location of blast wave formation is also important for proper placement of animal targets for experimental studies of blast-induced mTBI [99]. Existing shock tube experimental models have importantly identified pathological changes likely related to cardiovascular homeostatic mechanisms [78], as well as effects postulated to be due to electro-magnetic blast waves $[72,100]$; these efforts have been rewarding in advancing our knowledge of some aspects of blast injury. However, the shock tube models likely only partially imitate real-world conditions and most of them have tested at higher blast levels. Many rodent models exposed in compressed air-driven shock tubes sustain blast exposures with relatively low peak incident overpressures with large overpressure durations (greater than $10 \mathrm{~ms}$ ). By 
contrast, real-world exposure of an IED composed of stacked artillery shells [101] produces overpressure durations typically much less than $10 \mathrm{~ms}$ [102].

For denotation blast-driven shock tube configurations, the principles are the same as with compressed gas driven shock tube testing. The key difference between these two settings is that the blast shock tube uses a small explosive charge to generate high pressures rather than compressed gas $[85,103,104]$. The charge is detonated in the conical or parabolic driver section. Then the shock wave propagates down through the driven section as animal placed within the compressed gas tubes $[63,105,106]$. A singular disadvantage of the blast shock tubes is that explosive use requires proper handling along with additional safety and security measures. These barriers appear to be the main reason that investigators favor compressed gas driven shock tubes as compared to blast tubes or the more direct paradigm using open-field explosive blast testing.

In summary, while valuable data has been obtained, the nature of the shock tube configurations and the generation of complex shockwaves, conventional shock tubes may not model blast waves replicating those generated by free-field explosions. Reports describing shock tube apparatus and conditions, including but not limited to the design of the animal mounting rack and animal positioning (prone or supine), degree of head restraint, and relative location of the specimen to the tube, show considerable variation. These variations limit the extent of comparability and repeatability of experimental findings from different laboratories.

\subsubsection{Open-field blast settings}


In 2011, two reports from investigators in Singapore and Israel described the use of openfield blasts to study TBI in rats and mice $[79,80]$. This approach appears intuitively more attractive for study of actual blast exposures. We describe progress using a highly repeatable open-field blast mTBI mouse model to elucidate the physical blast properties and its resulting pathophysiologic effects particularly for mTBI. The main advantage of open-field blast testing is that it closely replicates real-world blast scenarios in terms of similar overpressures and blast wave duration [102]. But, as previously mentioned, explosive use poses particular problems. This type of modeling is expensive and time consuming; it is difficult to find a safe and accessible open-field blast site; explosives (such as 2,4,6-trinitrotoluene, TNT; or composition C-4) must be handled appropriately and stored in a secure environment, and finally, uncontrollable environmental conditions (such as wind, temperature and humidity) may influence the open-field blast setting and animal responses.

The Singapore report by Pun et al. demonstrated effects of a single sub-lethal blast over pressure exposure of either 7.1 PSI (48.9 $\mathrm{kPa})$ or $11.3 \mathrm{PSI}(77.3 \mathrm{kPa})$ upon rats in an open-field setting [80]. The animals were secured in metal cages that were anchored to the ground at the blast site and placed at either 24 or $30 \mathrm{~m}$ away from a TNT explosive source. A 0.4 by $0.4 \mathrm{~m}$ concrete block was interposed between the animals and the explosive source at a distance of $1.5 \mathrm{~m}$ from the animals to a shield against debris and protect animals against secondary blast injuries. These exposures resulted in petechiae and ecchymoses of lung tissues at day 4 and day 7 after blast. Darkened and shrunken neurons with the presence of perisomal spaces and narrowed vasculature at day 1 post blast as compared to control were found. However, this open-field setting presents 
serious drawbacks related to experimental blast design. First, the use of $120 \mathrm{~kg}$ of TNT resulted in mortality rates of $4.4 \%$ and $8.3 \%$ to model low- and high-level blast, respectively. Deaths in the blast groups revealed pulmonary hemorrhage post-mortem. Secondly, the block used to protect against secondary blast injuries may have interfered with the shock front and also have generated reflected waves or other exposure variations [81].

Rubovitch et al. modeled an open-field mouse blast of mTBI using $500 \mathrm{~g}$ of TNT [79]. Anesthetized mice were placed in individual compartments on a platform $1 \mathrm{~m}$ above the ground at distances of $4 \mathrm{~m}$ and $7 \mathrm{~m}$ and covered with a plastic mesh, which let the animals rest in their natural position facing toward the blast wave source. Their heads were unshielded. Side-on pencil gauge sensors were used to monitor the blast peak overpressure. The mice at a distance of $7 \mathrm{~m}$ were exposed to 2.5 PSI (17.2 kPa) peak overpressure; at a distance of $4 \mathrm{~m}$ they were exposed to $5.5 \mathrm{PSI}(37.9 \mathrm{kPa})$ peak overpressure. Fewer than $3 \%$ of the animals died as a consequence of these exposures and no gross pathological visual damage was recorded in internal organs 1 hour and 24 hours after blast. No alterations in basic neurological assessment or brain gross pathology were found acutely in the blast-exposed mice. However, cognitive deficits in novel object recognition (NOR) and Y maze tasks as well as axonal injuries persisted up to 30 days post blast. This open-field blast setting also had potential limitations regarding the animal holding compartment design. The animal holding compartment used in this study may block the primary blast wave transmission from the side and beneath. More importantly, the holding wall configuration might generate reflected pressure waves within each compartment, thus enhancing injury due to exposure to complex wave forms. The 
animals were placed on a holder, which may block additional reflected pressure from the "ground bounce" characteristic of open field blast injury [72, 107]. Following this original report, three research articles using this model have been published. One used matrix-assisted laser desorption/ionization (MALDI) mass spectrometry imaging to track the distribution of gangliosides in mouse brain tissue [108]. They observed major increases of the ganglioside GM2 in the hippocampus (dentate gyrus and CA3), thalamus (thalamic parafascicular/centromedian nuclei and the lateral thalamus), and hypothalamus after 2 and 24 hours (but not after 72 hours) of a single blast exposure of 2.5 and 5.5 PSI. The second report compared the weight-drop physical impact model to the open-field blast setting, and identified unique gene expression using bioinformatics array analysis [109]. This study showed that TBI in mice induced by either physical or blast injury, led to the development of marked deficits in NOR compared to sham control 7 days after TBI, however with no difference in Y maze performance. Gene expression regulation and molecular pathways connected to Alzheimer's disease displayed a markedly different form of regulation depending on the type of TBI by their observations. The third article reported that blast induced cognitive deficits were potentially attenuated by pre- or postinjury treatment with the glucagon-like peptide-1 receptor agonist, exendin-4 (Ex-4) [110]. Ex-4, administered pre- or post-injury, ameliorated blast-induced neurodegeneration as measured by the ratio of Fluoro Jade B (FJB)/neuronal nuclear antigen (NeuN) positive cells at 72 hours, memory deficits from days 7-14 (by NOR), and attenuated genes (Stmn1 most up-regulated and Megf 9 most down-regulated) regulated by blast at day 14 post-injury. 
Combined with WRAIR shock tube data, our review and current experience with our open-field model suggests existence of a threshold between 74.5 and $116.7 \mathrm{kPa}$ that separates low level blast from moderate to high level blast exposures. These exposures appear to be equivalent pathologically to human exposures ranging from moderate to severe TBI [111]. Studies from other laboratories using exposures of $74.5 \mathrm{kPa}$ or lower also appear to be consistent with the WRAIR findings. Exposures in rats up to $74.5 \mathrm{kPa}$ across a range of durations might reasonably be called low-level or mTBI blast exposure, causing only subtle or minor gross pathology, without lung trauma or death $[67,80,112$ $114]$.

\subsubsection{Behavioral changes and neuropathology of blast related mTBI}

We have searched and summarized original research articles focused on both shock tube and open-field blast setting using the PubMed database. Search terms included each of the terms "shock tube", "blast" or "open field blast" in combination with the terms "traumatic brain injury" or "brain trauma". No time limit was set with regard to publication date. Only English language articles were retrieved. Relevant articles related to blast TBI animal studies were selected on the basis of abstract review. Full articles were subsequently obtained and their references were searched for further relevant material. A total of 70 articles were selected and categorized into "low level blast impact (overpressure $<100 \mathrm{kPa}$ )", "intermediate level blast impact (overpressure $>100 \mathrm{kPa}$ and $<200 \mathrm{kPa}$ )", and "high level blast impact (overpressure > $200 \mathrm{kPa}$ )" (detailed references were listed in Table 2.1). Forty-six of these studies used rats (male Wistar, Long Evans Hooded, or Sprague-Dawley) and the majority of the others used mice. Compressed gas 
driven shock tube (helium, nitrogen, or other) was most often used to model blast exposures. Analysis of these publications revealed that the mortality rates for animals are significantly and positively correlated with the blast overpressure $(\mathrm{r}=0.6546, P<0.001)$ (Figure 2.1A). The mortality rate in both the low level and the intermediate level blast group was significantly lower than that occurring in the high level blast group (Figure 2.1B).
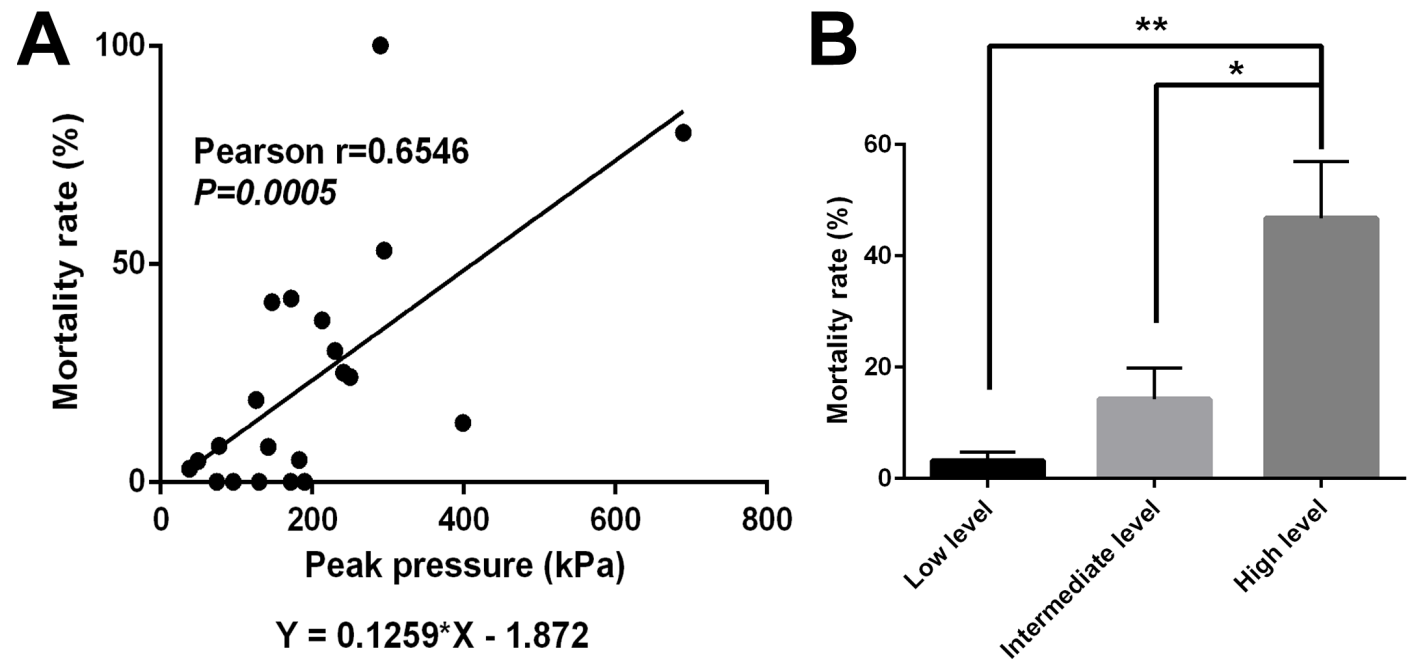

Figure 2.1 Correlation of mortality rate of animals with blast peak pressure and comparison of mortality rate among different levels of blast peak pressure.

(A) The mortality rate (\%) of experimental animals was significantly and positively correlated with the peak pressure $(\mathrm{kPa})$ of the blast. The Pearson correlation $\mathrm{r}=0.6546$ and the $P=0.0005$. The linear regression best-fit equation was "Mortality $(\%)=0.1259$ * peak overpressure $-1.872 "$. (B) The comparison of the mortality rate among level, intermediate, and high level of blast pressure. The statistics was calculated using one-way ANOVA test. The $P$ is 0.0025 . $(* P<0.05 ; * * P<0.01)$ 
Table 2.1 Summary of research articles of the blast-induced behavioral outcomes.

\begin{tabular}{|l|l|l|l|l|l|l|}
\hline \multirow{2}{*}{ Behavioral tests } & \multicolumn{2}{|c|}{ Low level } & \multicolumn{2}{c|}{ Intermediate } & \multicolumn{2}{c|}{ High level } \\
\cline { 2 - 8 } & $\begin{array}{r}\text { Short- } \\
\text { term }\end{array}$ & $\begin{array}{r}\text { Long- } \\
\text { term }\end{array}$ & $\begin{array}{r}\text { Short- } \\
\text { term }\end{array}$ & $\begin{array}{r}\text { Long- } \\
\text { term }\end{array}$ & $\begin{array}{r}\text { Short- } \\
\text { term }\end{array}$ & $\begin{array}{r}\text { Long- } \\
\text { term }\end{array}$ \\
\hline Motor & $2 / 6$ & $1 / 3$ & $3 / 5$ & $2 / 2$ & $1 / 1$ & $1 / 1$ \\
\hline Learning, memory, & $5 / 7$ & $5 / 5$ & $3 / 4$ & $5 / 5$ & $5 / 6$ & $3 / 4$ \\
and cognition & $2 / 4$ & $2 / 2$ & $3 / 5$ & $4 / 5$ & $3 / 4$ & $2 / 4$ \\
\hline Emotion and anxiety & $2 / 4$ & & & & &
\end{tabular}

Notes: Low level: overpressure $<100 \mathrm{kPa}$, references* $[67,69,77,79,80,95,108,109$, 112, 115-132]; Intermediate level: overpressure $>100 \mathrm{kPa}$ and $<200 \mathrm{kPa}$, references* [77, 90, 99, 115, 120, 121, 123, 130, 133-157]; High level: overpressure $>200 \mathrm{kPa}$, references* $[77,99,131,133,137-139,142,145,146,148,150,154,156-172]$. *, references cited here are also for Table 2 and Table 3.

Short-term: tests done within 7 days of injury; Long-term: tests done after 14 days of injury. Numbers are based on papers with deficits in tasks / total papers assessing tasks. Motor: rotarod test, forelimb grip-strength test, staircase test, balance beam task, openfield, general neurological assessment. Learning, memory, and cognition: passive and active avoidance tasks, novel object recognition, Y maze, Morris water maze, Barnes maze, fear conditioning. Emotion and anxiety: acoustic startle response, open-field, elevated plus maze, light-dark box. 
Based on the summary of the behavioral outcomes (Table 2.1), we identified that low, intermediate, and high blast levels induced various degrees of behavioral dysfunction in terms of motor, learning, memory, cognition, emotion, and anxiety. Interestingly, the short term (within 7 days of the blast injury) outcome measures showed more impairments as compared to the long term (after 14 days of the blast injury) observations. Significant behavioral changes as well as neuropathology of mTBI can be induced by low level blast exposures in experimental animals [111, 173]. In short term observations after a low level blast exposure, only 33\% (2/6), 50\% (2/4), and 71\% (5/7) of the papers described motor dysfunction, emotional disturbances, as well as learning, memory and cognitive disabilities, respectively. While in long term observations, $100 \%$ (8/8 and 3/3) of the research articles identified learning, memory, and emotional behavioral changes characteristic of mTBI. Open field task was also used to examine anxiety and spontaneous exploratory (locomotor) activity. Blast exposed animals were found to exhibit anxiety-like or stress-related behaviors [110, 127, 174, 175]. Findings from the Morris water maze documented impairments in spatial learning/memory defects $[145,176,177]$, which can also be observed clinically in mTBI [111]. Other motor, cognitive, and emotional changes due to blast injuries have been assessed by using NOR, passive avoidance, elevated plus maze, light-dark box test, among others (see details in Table 2.1). Blast related animals also showed cognitive and emotional changes both in short-term and long-term $[109,110,124]$. Extrapolation of these results to human behaviors requires nuanced interpretation, but these behavioral findings in low level blast exposed animals are clearly important. These models provide important clues and insights into brain injury mechanisms causing mTBI. 
Animal experiments also show that various degrees of eye, lung, and brain pathology occur in enhanced blast exposures. These also can be modeled using open-field blast exposure. Based on literature review, we note that at low level (mild) blast overpressure $(<100 \mathrm{kPa})$, less lung injury has been observed $(1 / 4)$ as compared to intermediate (4/4) and high level blast (6/6). While rarely identified in human combat blast, animals subjected to a blast wave (130-290 kPa) (intermediate to high levels) showed moderate pulmonary hemorrhage, associated vascular damage, with direct alveolar injury, and edema. Another report using more than $183 \mathrm{kPa}$ shock tube rupture pressure also showed that death occurred with extensive lung damage [36].

Blast exposure of animals also has induced tinnitus and central hearing impairment at a broad frequency range measured by gap detection and prepulse inhibition testing. These impairments tended to shift towards high frequencies over time [178]. Animal studies also confirmed greater degrees of pathological eye and brain outcomes (Table 2.2). Furthermore, our literature search indicated that cortical, hippocampal, and cerebellar tissue injuries were observed in all degrees of blast exposure along with hemorrhage/vascular injuries and white matter damage characterized as diffuse axonal injury (Table 2.3). The initial blast injury caused chronic changes in the microvasculature that were still evident many months after a $74.5 \mathrm{kPa}$ blast exposure [179]. Additionally, in low level TBI animal exposures, axonal degeneration can be identified using silver staining [177]. Diffusion tensor imaging of mTBI mouse brain in the original Rubovitch model has shown axonal and myelin abnormalities that require further study [79]. 
Table 2.2 Summary of the blast-induced macroscopic pathological outcomes.

\begin{tabular}{|l|l|l|l|}
\hline Pathology findings & Low level & Intermediate & High level \\
& & level & \\
\hline Lung & $1 / 4$ & $4 / 4$ & $6 / 6$ \\
\hline Eye & $1 / 1$ & $4 / 4$ & $3 / 3$ \\
\hline Brain & $25 / 25$ & $26 / 27$ & $27 / 27$ \\
\hline
\end{tabular}

Table 2.3 Summary of the blast-induced neuropathological outcomes.

\begin{tabular}{|l|l|l|l|}
\hline Neuropathology findings & Low level & Intermediate & High level \\
& & level & \\
\hline Cortex & $8 / 8$ & $7 / 7$ & $4 / 4$ \\
\hline Hippocampus & $4 / 4$ & $2 / 2$ & $4 / 4$ \\
\hline Cerebellum & $2 / 2$ & $3 / 3$ & $2 / 2$ \\
\hline Hemorrhage/Vascular injury & $5 / 7$ & $8 / 9$ & $5 / 6$ \\
\hline White matter damage & $10 / 10$ & $7 / 7$ & $5 / 5$ \\
\hline
\end{tabular}

The past decade of blast-induced TBI research yielded significant and novel findings concerning the physics of injury and its biological effects, particularly those that may cause mTBI. We have summarized underlying physical mechanisms of contemporary explosive devices and the spectrum of injuries inflicted by blasts / explosions, current shock tube and open-field mTBI animal models, and associated behavioral and neuropathological outcomes. Appropriate animal models of the blastinduced TBI will further deepen the understanding of physical mechanisms of the blast, 
and also assist in addressing the potential underlying mechanisms of the injury. On one hand, the majority of research articles on the blast-induced TBI used shock tube settings with various modifications; the data has provided useful information. However, openfield blast animal models have the unique ability to mimic actual blast explosions and multi-fold injuries occurring in modern combat exposures. We will in the next chapter describe a highly reproducible blast murine model of mTBI using open-field exposure to C4 explosives. The use of this open-field blast murine model may further establish relationships between the blast physical properties and $\mathrm{mTBI} /$ concussive injuries. Importantly, this model may also help to expand the available tools for researchers to study potential association between mTBI and later neurodegeneration. 


\subsection{REFERENCES}

[1] R.G. DePalma, D.G. Burris, H.R. Champion, M.J. Hodgson, Blast injuries, N Engl J Med 352(13) (2005) 1335-42.

[2] S.W. Hoffman, C. Harrison, The interaction between psychological health and traumatic brain injury: a neuroscience perspective, Clin Neuropsychol 23(8) (2009) 140015.

[3] R.L. Ruff, R.G. Riechers, 2nd, X.F. Wang, T. Piero, S.S. Ruff, A case-control study examining whether neurological deficits and PTSD in combat veterans are related to episodes of mild TBI, BMJ Open 2(2) (2012) e000312.

[4] R.G. DePalma, Combat TBI: History, Epidemiology, and Injury Modes(), in: F.H. Kobeissy (Ed.), Brain Neurotrauma: Molecular, Neuropsychological, and Rehabilitation Aspects, Boca Raton (FL), 2015.

[5] F.W. Mott, The effects of high explosives upon the central nervous system, Lancet 1(12) (1916) 331-8.

[6] F.W. Mott, The microscopic examination of the brains of two men dead of commotio cerebri (shell shock) without visible external injury, British medical journal 2(2967) (1917) 612 .

[7] J. Semmes, S. Weinstein, L. Ghent, H.L. Teuber, Performance on complex tactual tasks after brain injury in man: analyses by locus of lesion, Am J Psychol 67(2) (1954) $220-40$.

[8] M. Carey, W. Sacco, J. Merkler, An analysis of fatal and non-fatal head wounds incurred during combat in Vietnam by US forces, Acta chirurgica Scandinavica. Supplementum 508 (1981) 351-356. 
[9] J. Grafman, B.S. Jonas, A. Martin, A.M. Salazar, H. Weingartner, C. Ludlow, M.A. Smutok, S.C. Vance, Intellectual function following penetrating head injury in Vietnam veterans, Brain 111 ( Pt 1) (1988) 169-84.

[10] S.T. DeKosky, M.D. Ikonomovic, S. Gandy, Traumatic brain injury--football, warfare, and long-term effects, N Engl J Med 363(14) (2010) 1293-6.

[11] R.G. DePalma, G.M. Cross, L. Beck, D. Chandler, Epidemiology of mTBI (mild traumatic brain injury) due to blast: history, DOD/VA data bases: challenges and opportunities, Proceedings of the NATO RTO-MP-HFM-207 Symposium on A Survey of Blast Injury across the Full Landscape of Military Science, 2011, pp. 1-8.

[12] S.J. Bidelspach D, VA's TBI Screening and Evaluation Program.

$<$ http://www.hsrd.research.va.gov/for_researchers/cyber_seminars/archives/video_archiv e.cfm?SessionID=1085>, 2016 (accessed 25 Jan. 2016).

[13] J.V. Rosenfeld, A.C. McFarlane, P. Bragge, R.A. Armonda, J.B. Grimes, G.S. Ling, Blast-related traumatic brain injury, Lancet Neurol 12(9) (2013) 882-893.

[14] S. Weinberger, Bombs' hidden impact: the brain war, Nature 477(7365) (2011) 3903.

[15] A. Gawande, Casualties of war-military care for the wounded from Iraq and Afghanistan, New England Journal of Medicine 351(24) (2004) 2471-2475.

[16] S. Okie, Traumatic brain injury in the war zone, N Engl J Med 352(20) (2005) 20437.

[17] A.I. Schneiderman, E.R. Braver, H.K. Kang, Understanding sequelae of injury mechanisms and mild traumatic brain injury incurred during the conflicts in Iraq and 
Afghanistan: persistent postconcussive symptoms and posttraumatic stress disorder, Am J Epidemiol 167(12) (2008) 1446-52.

[18] S.B. Shively, I. Horkayne-Szakaly, R.V. Jones, J.P. Kelly, R.C. Armstrong, D.P. Perl, Characterisation of interface astroglial scarring in the human brain after blast exposure: a post-mortem case series, The Lancet Neurology (2016).

[19] W. Stewart, D.H. Smith, Time to be blunt about blast traumatic brain injury, Lancet Neurology 15(9) (2016) 896-898.

[20] C.L. Mac Donald, A.M. Johnson, D. Cooper, E.C. Nelson, N.J. Werner, J.S.

Shimony, A.Z. Snyder, M.E. Raichle, J.R. Witherow, R. Fang, Detection of blast-related traumatic brain injury in US military personnel, New England Journal of Medicine 364(22) (2011) 2091-2100.

[21] M. Balestreri, M. Czosnyka, D.A. Chatfield, L.A. Steiner, E.A. Schmidt, P.

Smielewski, B. Matta, J.D. Pickard, Predictive value of Glasgow Coma Scale after brain trauma: change in trend over the past ten years, J Neurol Neurosurg Psychiatry 75(1) (2004) 161-2.

[22] J. Head, Definition of mild traumatic brain.. Injury, J Head Trauma Rehabil 8(3) (1993) 86-87.

[23] R.M. Ruff, G.L. Iverson, J.T. Barth, S.S. Bush, D.K. Broshek, N.A.N. Policy, C. Planning, Recommendations for diagnosing a mild traumatic brain injury: a National Academy of Neuropsychology education paper, Arch Clin Neuropsychol 24(1) (2009) 310.

[24] G.A. Elder, E.M. Mitsis, S.T. Ahlers, A. Cristian, Blast-induced mild traumatic brain injury, Psychiatr Clin North Am 33(4) (2010) 757-81. 
[25] I. Cernak, L.J. Noble-Haeusslein, Traumatic brain injury: an overview of pathobiology with emphasis on military populations, Journal of Cerebral Blood Flow \& Metabolism 30(2) (2010) 255-266.

[26] H. Song, J. Cui, A. Simonyi, C.E. Johnson, G.K. Hubler, R.G. DePalma, Z. Gu, Linking blast physics to biological outcomes in mild traumatic brain injury: Narrative review and preliminary report of an open-field blast model, Behav Brain Res 340 (2018) $147-158$.

[27] H. Song, L.M. Konan, J. Cui, C.E. Johnson, G.K. Hubler, R.G. DePalma, Z. Gu, Nanometer ultrastructural brain damage following low intensity primary blast wave exposure, Neural Regen Res 13(9) (2018) 1516-1519.

[28] H. Song, L.M. Konan, J. Cui, C.E. Johnson, M. Langenderfer, D. Grant, T. Ndam, A. Simonyi, T. White, U. Demirci, D.R. Mott, D. Schwer, G.K. Hubler, I. Cernak, R.G. DePalma, Z. Gu, Ultrastructural brain abnormalities and associated behavioral changes in mice after low-intensity blast exposure, Behav Brain Res 347 (2018) 148-157.

[29] S. Okie, TBI's Long-Term Follow-up--Slow Progress in Science and Recovery, N Engl J Med 375(2) (2016) 180-4.

[30] A.I. Maas, N. Stocchetti, R. Bullock, Moderate and severe traumatic brain injury in adults, Lancet Neurol 7(8) (2008) 728-41.

[31] C.A. Luethcke, C.J. Bryan, C.E. Morrow, W.C. Isler, Comparison of Concussive Symptoms, Cognitive Performance, and Psychological Symptoms Between Acute BlastVersus Nonblast-Induced Mild Traumatic Brain Injury, Journal of the International Neuropsychological Society 17(1) (2011) 36-45. 
[32] A.P. Association, Diagnostic and statistical manual of mental disorders (DSM-5®), American Psychiatric Pub2013.

[33] A. Gawande, Casualties of war--military care for the wounded from Iraq and Afghanistan, N Engl J Med 351(24) (2004) 2471-5.

[34] S. Okie, Traumatic brain injury in the war zone, New England Journal of Medicine 352(20) (2005) 2043-2047.

[35] M.K. Nyein, A.M. Jason, L. Yu, C.M. Pita, J.D. Joannopoulos, D.F. Moore, R.A. Radovitzky, In silico investigation of intracranial blast mitigation with relevance to military traumatic brain injury, Proc Natl Acad Sci U S A 107(48) (2010) 20703-8. [36] I. Cernak, A.C. Merkle, V.E. Koliatsos, J.M. Bilik, Q.T. Luong, T.M. Mahota, L. $\mathrm{Xu}$, N. Slack, D. Windle, F.A. Ahmed, The pathobiology of blast injuries and blastinduced neurotrauma as identified using a new experimental model of injury in mice, Neurobiology of disease 41(2) (2011) 538-551.

[37] P.J. Belmont Jr, B.J. McCriskin, R.N. Sieg, R. Burks, A.J. Schoenfeld, Combat wounds in Iraq and Afghanistan from 2005 to 2009, Journal of trauma and acute care surgery 73(1) (2012) 3-12.

[38] C.E. Needham, Blast Wave Propagation, Blast Waves, Springer2010, pp. 87-99.

[39] N. Chandra, A. Sundaramurthy, Acute Pathophysiology of Blast Injury-From Biomechanics to Experiments and Computations: Implications on Head and Polytrauma, in: F.H. Kobeissy (Ed.), Brain Neurotrauma: Molecular, Neuropsychological, and Rehabilitation Aspects, Boca Raton (FL), 2015. 
[40] M.E. Carey, M. Herz, B. Corner, J. McEntire, D. Malabarba, S. Paquette, J.B.

Sampson, Ballistic helmets and aspects of their design, Neurosurgery 47(3) (2000) 678688.

[41] S.G. Mellor, G.J. Cooper, Analysis of 828 servicemen killed or injured by explosion in Northern Ireland 1970-84: the Hostile Action Casualty System, Br J Surg 76(10) (1989) 1006-10.

[42] G. Ling, F. Bandak, R. Armonda, G. Grant, J. Ecklund, Explosive blast neurotrauma, J Neurotrauma 26(6) (2009) 815-25.

[43] G.A. Elder, A. Cristian, Blast-related mild traumatic brain injury: mechanisms of injury and impact on clinical care, Mt Sinai J Med 76(2) (2009) 111-8.

[44] L. Young, G.T. Rule, R.T. Bocchieri, T.J. Walilko, J.M. Burns, G. Ling, When physics meets biology: low and high-velocity penetration, blunt impact, and blast injuries to the brain, Front Neurol 6 (2015) 89.

[45] C.R. Bass, M.B. Panzer, K.A. Rafaels, G. Wood, J. Shridharani, B. Capehart, Brain injuries from blast, Ann Biomed Eng 40(1) (2012) 185-202.

[46] R.N. Rogers, Thermochemistry of explosives, Thermochimica Acta 11(2) (1975) 131-139.

[47] M.A. Liberman, Introduction to physics and chemistry of combustion: explosion, flame, detonation, Springer Science \& Business Media2010.

[48] J.M. Dewey, The air velocity in blast waves from TNT explosions, Proceedings of the Royal Society of London A: Mathematical, Physical and Engineering Sciences, The Royal Society, 1964, pp. 366-385. 
[49] M.D. Goel, V.A. Matsagar, A.K. Gupta, S. Marburg, An Abridged Review of Blast Wave Parameters, Defence Science Journal 62(5) (2012) 300-306.

[50] G. Taylor, The formation of a blast wave by a very intense explosion. I. Theoretical discussion, Proceedings of the Royal Society of London. Series A, Mathematical and Physical Sciences (1950) 159-174.

[51] J.M. Dewey, The shape of the blast wave: studies of the Friedlander equation, Proceeding of the 21st International Symposium on Military Aspects of Blast and Shock (MABS), Israel, 2010, pp. 1-9.

[52] H.L. Brode, Numerical solutions of spherical blast waves, Journal of Applied physics 26(6) (1955) 766-775.

[53] J.M. Varas, M. Philippens, S. Meijer, A. Van Den Berg, P. Sibma, J. Van Bree, D. De Vries, Physics of IED blast shock tube simulations for mTBI research, Traumatic Injuries in the Nervous System (2011) 82.

[54] I. Cullis, Blast waves and how they interact with structures, Journal of the Royal Army Medical Corps 147(1) (2001) 16-26.

[55] K.H. Taber, D.L. Warden, R.A. Hurley, Blast-related traumatic brain injury: what is known?, The Journal of neuropsychiatry and clinical neurosciences (2006).

[56] C.-J. Clemedson, H. Pettersson, Propagation of a high explosive air shock wave through different parts of an animal body, American Journal of Physiology--Legacy Content 184(1) (1955) 119-126.

[57] A.C. Courtney, M.W. Courtney, A thoracic mechanism of mild traumatic brain injury due to blast pressure waves, Medical Hypotheses 72(1) (2009) 76-83.

[58] A. Holbourn, Mechanics of head injuries, The Lancet 242(6267) (1943) 438-441. 
[59] J.H. Stuhmiller, W. Santee, K. Friedl, Blast injury, translating research into operational medicine, Military Quantitative Physiology: Problems and Concepts in Military Operational Medicine (2008) 267-302.

[60] R.K. Gupta, A. Przekwas, Mathematical Models of Blast-Induced TBI: Current Status, Challenges, and Prospects, Front Neurol 4 (2013) 59.

[61] B. Engelhardt, L. Sorokin, The blood-brain and the blood-cerebrospinal fluid barriers: function and dysfunction, Seminars in immunopathology, Springer, 2009, pp. 497-511.

[62] V.E. Johnson, W. Stewart, D.H. Smith, Axonal pathology in traumatic brain injury, Exp Neurol 246 (2013) 35-43.

[63] B.D. Stemper, A.S. Shah, F.A. Pintar, M. McCrea, S.N. Kurpad, A. GlavaskiJoksimovic, C. Olsen, M.D. Budde, Head rotational acceleration characteristics influence behavioral and diffusion tensor imaging outcomes following concussion, Ann Biomed Eng 43(5) (2015) 1071-88.

[64] H. Zou, J.P. Schmiedeler, W.N. Hardy, Separating brain motion into rigid body displacement and deformation under low-severity impacts, J Biomech 40(6) (2007) 118391. [65] J.A. Forbes, S. Zuckerman, A.A. Abla, J. Mocco, K. Bode, T. Eads, Biomechanics of subdural hemorrhage in American football: review of the literature in response to rise in incidence, Childs Nerv Syst 30(2) (2014) 197-203.

[66] K.-U. Schmitt, P.F. Niederer, D.S. Cronin, M.H. Muser, F. Walz, Head injuries, Trauma Biomechanics, Springer2014, pp. 55-80. 
[67] E. Park, J.J. Gottlieb, B. Cheung, P.N. Shek, A.J. Baker, A model of low-level primary blast brain trauma results in cytoskeletal proteolysis and chronic functional impairment in the absence of lung barotrauma, J Neurotrauma 28(3) (2011) 343-57. [68] Y.P. Raizer, Physics of shock waves and high-temperature hydrodynamic phenomena, Courier Corporation2002.

[69] A. Saljo, B. Svensson, M. Mayorga, A. Hamberger, H. Bolouri, Low-level blasts raise intracranial pressure and impair cognitive function in rats, J Neurotrauma 26(8) (2009) 1345-52.

[70] Y. Kucherov, G.K. Hubler, R.G. DePalma, Blast induced mild traumatic brain injury/concussion: A physical analysis, Journal of Applied Physics 112(10) (2012) 104701.

[71] F.A. Duck, Physical properties of tissues: a comprehensive reference book, Academic press2013.

[72] I. Cernak, L.J. Noble-Haeusslein, Traumatic brain injury: an overview of pathobiology with emphasis on military populations, J Cereb Blood Flow Metab 30(2) (2010) 255-66.

[73] G.A. Elder, J.R. Stone, S.T. Ahlers, Effects of low-level blast exposure on the nervous system: is there really a controversy, Front Neurol 5(269.10) (2014) 3389.

[74] L. Galin, G. Cherepanov, Self-sustaining failure of a stressed brittle body, Soviet Physics Doklady, 1966, p. 267.

[75] L.C. Forde, W.G. Proud, S.M. Walley, P.D. Church, I.G. Cullis, Ballistic impact studies of a borosilicate glass, International Journal of Impact Engineering 37(5) (2010) 568-578. 
[76] Y. Kucherov, G. Hubler, J. Michopoulos, B. Johnson, Acoustic waves excited by phonon decay govern the fracture of brittle materials, Journal of Applied Physics 111(2) (2012) 023514 .

[77] K.A. Rafaels, C.R. Bass, M.B. Panzer, R.S. Salzar, W.A. Woods, S.H. Feldman, T. Walilko, R.W. Kent, B.P. Capehart, J.B. Foster, B. Derkunt, A. Toman, Brain injury risk from primary blast, J Trauma Acute Care Surg 73(4) (2012) 895-901.

[78] J.B. Long, T.L. Bentley, K.A. Wessner, C. Cerone, S. Sweeney, R.A. Bauman, Blast overpressure in rats: recreating a battlefield injury in the laboratory, Journal of neurotrauma 26(6) (2009) 827-840.

[79] V. Rubovitch, M. Ten-Bosch, O. Zohar, C.R. Harrison, C. Tempel-Brami, E. Stein, B.J. Hoffer, C.D. Balaban, S. Schreiber, W.T. Chiu, C.G. Pick, A mouse model of blastinduced mild traumatic brain injury, Exp Neurol 232(2) (2011) 280-9.

[80] P.B. Pun, E.M. Kan, A. Salim, Z. Li, K.C. Ng, S.M. Moochhala, E.A. Ling, M.H. Tan, J. Lu, Low level primary blast injury in rodent brain, Front Neurol 2 (2011) 19. [81] A. Nakagawa, G.T. Manley, A.D. Gean, K. Ohtani, R. Armonda, A. Tsukamoto, H. Yamamoto, K. Takayama, T. Tominaga, Mechanisms of primary blast-induced traumatic brain injury: insights from shock-wave research, J Neurotrauma 28(6) (2011) 1101-19. [82] F. Kobeissy, S. Mondello, N. Tumer, H.Z. Toklu, M.A. Whidden, N. Kirichenko, Z. Zhang, V. Prima, W. Yassin, J. Anagli, N. Chandra, S. Svetlov, K.K. Wang, Assessing neuro-systemic \& behavioral components in the pathophysiology of blast-related brain injury, Front Neurol 4 (2013) 186.

[83] M.S. Chafi, G. Karami, M. Ziejewski, Biomechanical assessment of brain dynamic responses due to blast pressure waves, Ann Biomed Eng 38(2) (2010) 490-504. 
[84] A. Jean, M.K. Nyein, J.Q. Zheng, D.F. Moore, J.D. Joannopoulos, R. Radovitzky, An animal-to-human scaling law for blast-induced traumatic brain injury risk assessment, Proc Natl Acad Sci U S A 111(43) (2014) 15310-5.

[85] Y. Xiong, A. Mahmood, M. Chopp, Animal models of traumatic brain injury, Nat Rev Neurosci 14(2) (2013) 128-42.

[86] D. Richmond, M. Wetherbe, R. Taborelli, R. Sanchez, F. Sherping, Shock tube studies of the effects of sharp-rising, long-duration overpressures on biological systems, DTIC Document, 1959.

[87] Y. Chen, W. Huang, Non-impact, blast-induced mild TBI and PTSD: concepts and caveats, Brain Inj 25(7-8) (2011) 641-50.

[88] Y. Chen, S. Constantini, Caveats for using shock tube in blast-induced traumatic brain injury research, Front Neurol 4 (2013) 117.

[89] H. Celander, C.J. Clemedson, U.A. Ericsson, H.I. Hultman, The use of a compressed air operated shock tube for physiological blast research, Acta physiologica Scandinavica 33(1) (1955) 6-13.

[90] D.V. Reneer, R.D. Hisel, J.M. Hoffman, R.J. Kryscio, B.T. Lusk, J.W. Geddes, A multi-mode shock tube for investigation of blast-induced traumatic brain injury, $\mathrm{J}$ Neurotrauma 28(1) (2011) 95-104.

[91] C.E. Needham, D. Ritzel, G.T. Rule, S. Wiri, L. Young, Blast testing issues and TBI: experimental models that lead to wrong conclusions, Frontiers in neurology 6 (2015).

[92] A.D. Leonardi, C.A. Bir, D.V. Ritzel, P.J. VandeVord, Intracranial pressure increases during exposure to a shock wave, J Neurotrauma 28(1) (2011) 85-94. 
[93] I. Cernak, J. Savic, Z. Malicevic, G. Zunic, P. Radosevic, I. Ivanovic, L. Davidovic, Involvement of the central nervous system in the general response to pulmonary blast injury, Journal of Trauma and Acute Care Surgery 40(3S) (1996) 100S-104S.

[94] I. Cernak, Z. Wang, J. Jiang, X. Bian, J. Savic, Ultrastructural and functional characteristics of blast injury-induced neurotrauma, Journal of Trauma and Acute Care Surgery 50(4) (2001) 695-706.

[95] G.A. Elder, N.P. Dorr, R. De Gasperi, M.A. Gama Sosa, M.C. Shaughness, E. Maudlin-Jeronimo, A.A. Hall, R.M. McCarron, S.T. Ahlers, Blast exposure induces posttraumatic stress disorder-related traits in a rat model of mild traumatic brain injury, $\mathrm{J}$ Neurotrauma 29(16) (2012) 2564-75.

[96] B.R. Huber, J.S. Meabon, T.J. Martin, P.D. Mourad, R. Bennett, B.C. Kraemer, I. Cernak, E.C. Petrie, M.J. Emery, E.R. Swenson, C. Mayer, E. Mehic, E.R. Peskind, D.G. Cook, Blast exposure causes early and persistent aberrant phospho- and cleaved-tau expression in a murine model of mild blast-induced traumatic brain injury, J Alzheimers Dis 37(2) (2013) 309-23.

[97] T. Sawyer, Y. Wang, D.V. Ritzel, T. Josey, M. Villanueva, Y. Shei, P. Nelson, G. Hennes, T. Weiss, C. Vair, High Fidelity Simulation of Primary Blast: Direct Effects on the Head, Journal of Neurotrauma (ja) (2015).

[98] A.F. Tasissa, M. Hautefeuille, J.H. Fitek, R.A. Radovitzky, On the formation of Friedlander waves in a compressed-gas-driven shock tube, Proc Math Phys Eng Sci 472(2186) (2016) 20150611. 
[99] A. Sundaramurthy, A. Alai, S. Ganpule, A. Holmberg, E. Plougonven, N. Chandra, Blast-induced biomechanical loading of the rat: an experimental and anatomically accurate computational blast injury model, J Neurotrauma 29(13) (2012) 2352-64. [100] J. Cheng, J. Gu, Y. Ma, T. Yang, Y. Kuang, B. Li, J. Kang, Development of a rat model for studying blast-induced traumatic brain injury, J Neurol Sci 294(1-2) (2010) 238.

[101] T.J. Nelson, T. Clark, E.T. Stedje-Larsen, C.T. Lewis, J.M. Grueskin, E.L. Echols, D.B. Wall, E.A. Felger, H.R. Bohman, Close proximity blast injury patterns from improvised explosive devices in Iraq: a report of 18 cases, J Trauma 65(1) (2008) 212-7. [102] M.B. Panzer, B.S. Myers, B.P. Capehart, C.R. Bass, Development of a finite element model for blast brain injury and the effects of CSF cavitation, Ann Biomed Eng 40(7) (2012) 1530-44.

[103] M. Risling, S. Plantman, M. Angeria, E. Rostami, B.M. Bellander, M. Kirkegaard, U. Arborelius, J. Davidsson, Mechanisms of blast induced brain injuries, experimental studies in rats, Neuroimage 54 Suppl 1 (2011) S89-97. [104] N.C. de Lanerolle, F. Bandak, D. Kang, A.Y. Li, F. Du, P. Swauger, S. Parks, G. Ling, J.H. Kim, Characteristics of an explosive blast-induced brain injury in an experimental model, J Neuropathol Exp Neurol 70(11) (2011) 1046-57. [105] R. Kuehn, P.F. Simard, I. Driscoll, K. Keledjian, S. Ivanova, C. Tosun, A. Williams, G. Bochicchio, V. Gerzanich, J.M. Simard, Rodent model of direct cranial blast injury, J Neurotrauma 28(10) (2011) 2155-69. 
[106] A. Gyorgy, G. Ling, D. Wingo, J. Walker, L. Tong, S. Parks, A. Januszkiewicz, R. Baumann, D.V. Agoston, Time-dependent changes in serum biomarker levels after blast traumatic brain injury, J Neurotrauma 28(6) (2011) 1121-6.

[107] G. Ben-Dor, O. Igra, T. Elperin, Handbook of Shock Waves, Three Volume Set, Academic Press2000.

[108] A.S. Woods, B. Colsch, S.N. Jackson, J. Post, K. Baldwin, A. Roux, B. Hoffer, B.M. Cox, M. Hoffer, V. Rubovitch, C.G. Pick, J.A. Schultz, C. Balaban, Gangliosides and ceramides change in a mouse model of blast induced traumatic brain injury, ACS Chem Neurosci 4(4) (2013) 594-600.

[109] D. Tweedie, L. Rachmany, V. Rubovitch, Y. Zhang, K.G. Becker, E. Perez, B.J. Hoffer, C.G. Pick, N.H. Greig, Changes in mouse cognition and hippocampal gene expression observed in a mild physical- and blast-traumatic brain injury, Neurobiol Dis $54(2013) 1-11$.

[110] D. Tweedie, L. Rachmany, V. Rubovitch, Y. Li, H.W. Holloway, E. Lehrmann, Y. Zhang, K.G. Becker, E. Perez, B.J. Hoffer, Blast traumatic brain injury-induced cognitive deficits are attenuated by preinjury or postinjury treatment with the glucagonlike peptide-1 receptor agonist, exendin-4, Alzheimer's \& Dementia 12(1) (2016) 34-48. [111] S.T. Ahlers, E. Vasserman-Stokes, M.C. Shaughness, A.A. Hall, D.A. Shear, M. Chavko, R.M. McCarron, J.R. Stone, Assessment of the effects of acute and repeated exposure to blast overpressure in rodents: toward a greater understanding of blast and the potential ramifications for injury in humans exposed to blast, Frontiers in neurology 3 (2012). 
[112] A. Saljo, H. Bolouri, M. Mayorga, B. Svensson, A. Hamberger, Low-level blast raises intracranial pressure and impairs cognitive function in rats: prophylaxis with processed cereal feed, J Neurotrauma 27(2) (2010) 383-9.

[113] A. Saljo, M. Mayorga, H. Bolouri, B. Svensson, A. Hamberger, Mechanisms and pathophysiology of the low-level blast brain injury in animal models, Neuroimage 54 Suppl 1 (2011) S83-8.

[114] K.L. Baalman, R.J. Cotton, S.N. Rasband, M.N. Rasband, Blast wave exposure impairs memory and decreases axon initial segment length, Journal of neurotrauma 30(9) (2013) 741-751.

[115] J.M. Petras, R.A. Bauman, N.M. Elsayed, Visual system degeneration induced by blast overpressure, Toxicology 121(1) (1997) 41-9.

[116] S.M. Moochhala, S. Md, J. Lu, C.H. Teng, C. Greengrass, Neuroprotective role of aminoguanidine in behavioral changes after blast injury, J Trauma 56(2) (2004) 393-403. [117] M. Chavko, W.A. Koller, W.K. Prusaczyk, R.M. McCarron, Measurement of blast wave by a miniature fiber optic pressure transducer in the rat brain, J Neurosci Methods 159(2) (2007) 277-81.

[118] A. Saljo, F. Arrhen, H. Bolouri, M. Mayorga, A. Hamberger, Neuropathology and pressure in the pig brain resulting from low-impulse noise exposure, J Neurotrauma 25(12) (2008) 1397-406.

[119] M. Chavko, T. Watanabe, S. Adeeb, J. Lankasky, S.T. Ahlers, R.M. McCarron, Relationship between orientation to a blast and pressure wave propagation inside the rat brain, J Neurosci Methods 195(1) (2011) 61-6. 
[120] Y. Wang, Y. Wei, S. Oguntayo, W. Wilkins, P. Arun, M. Valiyaveettil, J. Song, J.B. Long, M.P. Nambiar, Tightly coupled repetitive blast-induced traumatic brain injury: development and characterization in mice, J Neurotrauma 28(10) (2011) 2171-83. [121] S.T. Ahlers, E. Vasserman-Stokes, M.C. Shaughness, A.A. Hall, D.A. Shear, M. Chavko, R.M. McCarron, J.R. Stone, Assessment of the effects of acute and repeated exposure to blast overpressure in rodents: toward a greater understanding of blast and the potential ramifications for injury in humans exposed to blast, Front Neurol 3 (2012) 32. [122] D.L. Ewert, J. Lu, W. Li, X. Du, R. Floyd, R. Kopke, Antioxidant treatment reduces blast-induced cochlear damage and hearing loss, Hear Res 285(1-2) (2012) 2939.

[123] R. De Gasperi, M.A. Gama Sosa, S.H. Kim, J.W. Steele, M.C. Shaughness, E. Maudlin-Jeronimo, A.A. Hall, S.T. Dekosky, R.M. McCarron, M.P. Nambiar, S. Gandy, S.T. Ahlers, G.A. Elder, Acute blast injury reduces brain abeta in two rodent species, Front Neurol 3 (2012) 177.

[124] L.E. Goldstein, A.M. Fisher, C.A. Tagge, X.L. Zhang, L. Velisek, J.A. Sullivan, C. Upreti, J.M. Kracht, M. Ericsson, M.W. Wojnarowicz, C.J. Goletiani, G.M. Maglakelidze, N. Casey, J.A. Moncaster, O. Minaeva, R.D. Moir, C.J. Nowinski, R.A. Stern, R.C. Cantu, J. Geiling, J.K. Blusztajn, B.L. Wolozin, T. Ikezu, T.D. Stein, A.E. Budson, N.W. Kowall, D. Chargin, A. Sharon, S. Saman, G.F. Hall, W.C. Moss, R.O. Cleveland, R.E. Tanzi, P.K. Stanton, A.C. McKee, Chronic traumatic encephalopathy in blast-exposed military veterans and a blast neurotrauma mouse model, Sci Transl Med 4(134) (2012) 134ra60. 
[125] M.A. Sosa, R. De Gasperi, A.J. Paulino, P.E. Pricop, M.C. Shaughness, E. Maudlin-Jeronimo, A.A. Hall, W.G. Janssen, F.J. Yuk, N.P. Dorr, D.L. Dickstein, R.M. McCarron, M. Chavko, P.R. Hof, S.T. Ahlers, G.A. Elder, Blast overpressure induces shear-related injuries in the brain of rats exposed to a mild traumatic brain injury, Acta Neuropathol Commun 1 (2013) 51.

[126] K.L. Baalman, R.J. Cotton, S.N. Rasband, M.N. Rasband, Blast wave exposure impairs memory and decreases axon initial segment length, J Neurotrauma 30(9) (2013) $741-51$.

[127] K. Xie, H. Kuang, J.Z. Tsien, Mild blast events alter anxiety, memory, and neural activity patterns in the anterior cingulate cortex, PLoS One 8(5) (2013) e64907. [128] R.F. Genovese, L.P. Simmons, S.T. Ahlers, E. Maudlin-Jeronimo, J.R. Dave, A.M. Boutte, Effects of mild TBI from repeated blast overpressure on the expression and extinction of conditioned fear in rats, Neuroscience 254 (2013) 120-9. [129] M.A. Gama Sosa, R. De Gasperi, P.L. Janssen, F.J. Yuk, P.C. Anazodo, P.E. Pricop, A.J. Paulino, B. Wicinski, M.C. Shaughness, E. Maudlin-Jeronimo, A.A. Hall, D.L. Dickstein, R.M. McCarron, M. Chavko, P.R. Hof, S.T. Ahlers, G.A. Elder, Selective vulnerability of the cerebral vasculature to blast injury in a rat model of mild traumatic brain injury, Acta Neuropathol Commun 2 (2014) 67.

[130] S. Kallakuri, H.S. Purkait, S. Dalavayi, P. VandeVord, J.M. Cavanaugh, Blast overpressure induced axonal injury changes in rat brainstem and spinal cord, J Neurosci Rural Pract 6(4) (2015) 481-7. 
[131] H. Wang, Y.P. Zhang, J. Cai, L.B. Shields, C.A. Tuchek, R. Shi, J. Li, C.B.

Shields, X.M. Xu, A Compact Blast-Induced Traumatic Brain Injury Model in Mice, J Neuropathol Exp Neurol (2016).

[132] D. Tweedie, L. Rachmany, V. Rubovitch, Y. Li, H.W. Holloway, E. Lehrmann, Y. Zhang, K.G. Becker, E. Perez, B.J. Hoffer, C.G. Pick, N.H. Greig, Blast traumatic brain injury-induced cognitive deficits are attenuated by preinjury or postinjury treatment with the glucagon-like peptide-1 receptor agonist, exendin-4, Alzheimers Dement 12(1) (2016) 34-48.

[133] Z. Wang, L. Sun, Z. Yang, H. Leng, J. Jiang, H. Yu, J. Gu, Z. Li, Development of serial bio-shock tubes and their application, Chin Med J (Engl) 111(2) (1998) 109-13. [134] M. Chavko, W.K. Prusaczyk, R.M. McCarron, Lung injury and recovery after exposure to blast overpressure, J Trauma 61(4) (2006) 933-42.

[135] J.B. Long, T.L. Bentley, K.A. Wessner, C. Cerone, S. Sweeney, R.A. Bauman, Blast overpressure in rats: recreating a battlefield injury in the laboratory, J Neurotrauma 26(6) (2009) 827-40.

[136] R.D. Readnower, M. Chavko, S. Adeeb, M.D. Conroy, J.R. Pauly, R.M. McCarron, P.G. Sullivan, Increase in blood-brain barrier permeability, oxidative stress, and activated microglia in a rat model of blast-induced traumatic brain injury, J Neurosci Res $88(16)$ (2010) 3530-9.

[137] I. Cernak, A.C. Merkle, V.E. Koliatsos, J.M. Bilik, Q.T. Luong, T.M. Mahota, L. Xu, N. Slack, D. Windle, F.A. Ahmed, The pathobiology of blast injuries and blastinduced neurotrauma as identified using a new experimental model of injury in mice, Neurobiol Dis 41(2) (2011) 538-51. 
[138] K. Rafaels, C.R. Bass, R.S. Salzar, M.B. Panzer, W. Woods, S. Feldman, T. Cummings, B. Capehart, Survival risk assessment for primary blast exposures to the head, J Neurotrauma 28(11) (2011) 2319-28.

[139] V.E. Koliatsos, I. Cernak, L. Xu, Y. Song, A. Savonenko, B.J. Crain, C.G.

Eberhart, C.E. Frangakis, T. Melnikova, H. Kim, D. Lee, A mouse model of blast injury to brain: initial pathological, neuropathological, and behavioral characterization, $\mathrm{J}$ Neuropathol Exp Neurol 70(5) (2011) 399-416.

[140] D.K. Cullen, K.D. Browne, Y. Xu, S. Adeeb, J.A. Wolf, R.M. McCarron, S. Yang, M. Chavko, D.H. Smith, Blast-induced color change in photonic crystals corresponds with brain pathology, J Neurotrauma 28(11) (2011) 2307-18.

[141] J.J. Dalle Lucca, M. Chavko, M.A. Dubick, S. Adeeb, M.J. Falabella, J.L. Slack, R. McCarron, Y. Li, Blast-induced moderate neurotrauma (BINT) elicits early complement activation and tumor necrosis factor alpha (TNFalpha) release in a rat brain, J Neurol Sci 318(1-2) (2012) 146-54.

[142] J.K. Shridharani, G.W. Wood, M.B. Panzer, B.P. Capehart, M.K. Nyein, R.A.

Radovitzky, C.R. Bass, Porcine head response to blast, Front Neurol 3 (2012) 70.

[143] E. Kovesdi, A. Kamnaksh, D. Wingo, F. Ahmed, N.E. Grunberg, J.B. Long, C.E.

Kasper, D.V. Agoston, Acute minocycline treatment mitigates the symptoms of mild blast-induced traumatic brain injury, Front Neurol 3 (2012) 111.

[144] E. Huang, M. Ngo, S. Yee, L. Held, K. Norman, A.M. Scremin, O. Scremin, Repeated blast exposure alters open field behavior recorded under low illumination, Brain Res 1529 (2013) 125-33. 
[145] M.D. Budde, A. Shah, M. McCrea, W.E. Cullinan, F.A. Pintar, B.D. Stemper, Primary blast traumatic brain injury in the rat: relating diffusion tensor imaging and behavior, Front Neurol 4 (2013) 154.

[146] M. Skotak, F. Wang, A. Alai, A. Holmberg, S. Harris, R.C. Switzer, N. Chandra, Rat injury model under controlled field-relevant primary blast conditions: acute response to a wide range of peak overpressures, J Neurotrauma 30(13) (2013) 1147-60.

[147] P.M. Abdul-Muneer, H. Schuetz, F. Wang, M. Skotak, J. Jones, S. Gorantla, M.C. Zimmerman, N. Chandra, J. Haorah, Induction of oxidative and nitrosative damage leads to cerebrovascular inflammation in an animal model of mild traumatic brain injury induced by primary blast, Free Radic Biol Med 60 (2013) 282-91.

[148] S. Yeoh, E.D. Bell, K.L. Monson, Distribution of blood-brain barrier disruption in primary blast injury, Ann Biomed Eng 41(10) (2013) 2206-14 .

[149] P. Arun, R. Abu-Taleb, S. Oguntayo, Y. Wang, M. Valiyaveettil, J.B. Long, M.P. Nambiar, Acute mitochondrial dysfunction after blast exposure: potential role of mitochondrial glutamate oxaloacetate transaminase, J Neurotrauma 30(19) (2013) 164551. [150] S.A. Heldt, A.J. Elberger, Y. Deng, N.H. Guley, N. Del Mar, J. Rogers, G.W. Choi, J. Ferrell, T.S. Rex, M.G. Honig, A. Reiner, A novel closed-head model of mild traumatic brain injury caused by primary overpressure blast to the cranium produces sustained emotional deficits in mice, Front Neurol 5 (2014) 2.

[151] H.C. Wang, J.H. Choi, W.A. Greene, M.L. Plamper, H.E. Cortez, M. Chavko, Y. Li, J.J. Dalle Lucca, A.J. Johnson, Pathophysiology of blast-induced ocular trauma with apoptosis in the retina and optic nerve, Mil Med 179(8 Suppl) (2014) 34-40. 
[152] T.C. Yin, J.K. Britt, H. De Jesus-Cortes, Y. Lu, R.M. Genova, M.Z. Khan, J.R. Voorhees, J. Shao, A.C. Katzman, P.J. Huntington, C. Wassink, L. McDaniel, E.A. Newell, L.M. Dutca, J. Naidoo, H. Cui, A.G. Bassuk, M.M. Harper, S.L. McKnight, J.M. Ready, A.A. Pieper, P7C3 neuroprotective chemicals block axonal degeneration and preserve function after traumatic brain injury, Cell Rep 8(6) (2014) 1731-1740.

[153] S.K. Verma, E.M. Kan, J. Lu, K.C. Ng, E.A. Ling, S. Seramani, B.P. Kn, Y.C. Wong, M.H. Tan, S.S. Velan, Multi-echo susceptibility-weighted imaging and histology of open-field blast-induced traumatic brain injury in a rat model, NMR Biomed 28(9) (2015) 1069-77.

[154] S. Kabu, H. Jaffer, M. Petro, D. Dudzinski, D. Stewart, A. Courtney, M. Courtney, V. Labhasetwar, Blast-Associated Shock Waves Result in Increased Brain Vascular Leakage and Elevated ROS Levels in a Rat Model of Traumatic Brain Injury, PLoS One 10(5) (2015) e0127971.

[155] M.K. Walls, N. Race, L. Zheng, S.M. Vega-Alvarez, G. Acosta, J. Park, R. Shi, Structural and biochemical abnormalities in the absence of acute deficits in mild primary blast-induced head trauma, J Neurosurg 124(3) (2016) 675-86.

[156] T.W. Sawyer, Y. Wang, D.V. Ritzel, T. Josey, M. Villanueva, Y. Shei, P. Nelson, G. Hennes, T. Weiss, C. Vair, C. Fan, J. Barnes, High-Fidelity Simulation of Primary Blast: Direct Effects on the Head, J Neurotrauma 33(13) (2016) 1181-93.

[157] L. Xu, M.L. Schaefer, R.M. Linville, A. Aggarwal, W. Mbuguiro, B.A. Wester, V.E. Koliatsos, Neuroinflammation in primary blast neurotrauma: Time course and prevention by torso shielding, Exp Neurol 277 (2016) 268-274. 
[158] I. Cernak, P. Radosevic, Z. Malicevic, J. Savic, Experimental magnesium depletion in adult rabbits caused by blast overpressure, Magnes Res 8(3) (1995) 249-59.

[159] I. Cernak, J. Savic, Z. Malicevic, G. Zunic, P. Radosevic, I. Ivanovic, L. Davidovic, Involvement of the central nervous system in the general response to pulmonary blast injury, J Trauma 40(3 Suppl) (1996) S100-4.

[160] I. Cernak, Z. Wang, J. Jiang, X. Bian, J. Savic, Ultrastructural and functional characteristics of blast injury-induced neurotrauma, J Trauma 50(4) (2001) 695-706. [161] I. Cernak, Z. Wang, J. Jiang, X. Bian, J. Savic, Cognitive deficits following blast injury-induced neurotrauma: possible involvement of nitric oxide, Brain Inj 15(7) (2001) 593-612.

[162] R.H. Garman, L.W. Jenkins, R.C. Switzer, 3rd, R.A. Bauman, L.C. Tong, P.V. Swauger, S.A. Parks, D.V. Ritzel, C.E. Dixon, R.S. Clark, H. Bayir, V. Kagan, E.K. Jackson, P.M. Kochanek, Blast exposure in rats with body shielding is characterized primarily by diffuse axonal injury, J Neurotrauma 28(6) (2011) 947-59.

[163] S.I. Svetlov, V. Prima, O. Glushakova, A. Svetlov, D.R. Kirk, H. Gutierrez, V.L. Serebruany, K.C. Curley, K.K. Wang, R.L. Hayes, Neuro-glial and systemic mechanisms of pathological responses in rat models of primary blast overpressure compared to "composite" blast, Front Neurol 3 (2012) 15.

[164] S. Ganpule, A. Alai, E. Plougonven, N. Chandra, Mechanics of blast loading on the head models in the study of traumatic brain injury using experimental and computational approaches, Biomech Model Mechanobiol 12(3) (2013) 511-31.

[165] P.M. Kochanek, C.E. Dixon, D.K. Shellington, S.S. Shin, H. Bayir, E.K. Jackson, V.E. Kagan, H.Q. Yan, P.V. Swauger, S.A. Parks, D.V. Ritzel, R. Bauman, R.S. Clark, 
R.H. Garman, F. Bandak, G. Ling, L.W. Jenkins, Screening of biochemical and molecular mechanisms of secondary injury and repair in the brain after experimental blast-induced traumatic brain injury in rats, J Neurotrauma 30(11) (2013) 920-37. [166] N. Tumer, S. Svetlov, M. Whidden, N. Kirichenko, V. Prima, B. Erdos, A. Sherman, F. Kobeissy, R. Yezierski, P.J. Scarpace, C. Vierck, K.K. Wang, Overpressure blast-wave induced brain injury elevates oxidative stress in the hypothalamus and catecholamine biosynthesis in the rat adrenal medulla, Neurosci Lett 544 (2013) 62-7. [167] R.C. Turner, Z.J. Naser, A.F. Logsdon, K.H. DiPasquale, G.J. Jackson, M.J. Robson, R.T. Gettens, R.R. Matsumoto, J.D. Huber, C.L. Rosen, Modeling clinically relevant blast parameters based on scaling principles produces functional \& histological deficits in rats, Exp Neurol 248 (2013) 520-9.

[168] C.D. Hue, F.S. Cho, S. Cao, R.E. Nicholls, E.W. Vogel Iii, C. Sibindi, O. Arancio, C.R. Dale Bass, D.F. Meaney, B. Morrison Iii, Time Course and Size of Blood-Brain Barrier Opening in a Mouse Model of Blast-Induced Traumatic Brain Injury, J Neurotrauma 33(13) (2016) 1202-11.

[169] O. Rodriguez, M.L. Schaefer, B. Wester, Y.C. Lee, N. Boggs, H.A. Conner, A.C. Merkle, S.T. Fricke, C. Albanese, V.E. Koliatsos, Manganese-Enhanced Magnetic Resonance Imaging as a Diagnostic and Dispositional Tool after Mild-Moderate Blast Traumatic Brain Injury, J Neurotrauma 33(7) (2016) 662-71.

[170] B.D. Stemper, A.S. Shah, M.D. Budde, C.M. Olsen, A. Glavaski-Joksimovic, S.N. Kurpad, M. McCrea, F.A. Pintar, Behavioral Outcomes Differ between Rotational Acceleration and Blast Mechanisms of Mild Traumatic Brain Injury, Front Neurol 7 (2016) 31 . 
[171] A. Nakagawa, K. Ohtani, K. Goda, D. Kudo, T. Arafune, T. Washio, T. Tominaga, Mechanism of Traumatic Brain Injury at Distant Locations After Exposure to Blast Waves: Preliminary Results from Animal and Phantom Experiments, Acta Neurochir Suppl 122 (2016) 3-7.

[172] M. Beamer, S.R. Tummala, D. Gullotti, C. Kopil, S. Gorka, A. Ted, C.R. Bass, B. Morrison, 3rd, A.S. Cohen, D.F. Meaney, Primary blast injury causes cognitive impairments and hippocampal circuit alterations, Exp Neurol 283(Pt A) (2016) 16-28. [173] M.A. Sosa, R. De Gasperi, A.J. Paulino, P.E. Pricop, M.C. Shaughness, E. Maudlin-Jeronimo, A.A. Hall, W.G. Janssen, F.J. Yuk, N.P. Dorr, D.L. Dickstein, R.M. McCarron, M. Chavko, P.R. Hof, S.T. Ahlers, G.A. Elder, Blast overpressure induces shear-related injuries in the brain of rats exposed to a mild traumatic brain injury, Acta Neuropathol Commun 1(1) (2013) 51.

[174] P.M. Washington, P.A. Forcelli, T. Wilkins, D.N. Zapple, M. Parsadanian, M.P. Burns, The effect of injury severity on behavior: a phenotypic study of cognitive and emotional deficits after mild, moderate, and severe controlled cortical impact injury in mice, J Neurotrauma 29(13) (2012) 2283-96.

[175] E. Park, R. Eisen, A. Kinio, A.J. Baker, Electrophysiological white matter dysfunction and association with neurobehavioral deficits following low-level primary blast trauma, Neurobiol Dis 52 (2013) 150-9.

[176] J. Zhuo, K. Keledjian, S. Xu, A. Pampori, V. Gerzanich, J.M. Simard, R.P. Gullapalli, Changes in Diffusion Kurtosis Imaging and Magnetic Resonance Spectroscopy in a Direct Cranial Blast Traumatic Brain Injury (dc-bTBI) Model, PLoS One 10(8) (2015) e0136151. 
[177] H. Wang, Y.P. Zhang, J. Cai, L.B. Shields, C.A. Tuchek, R. Shi, J. Li, C.B. Shields, X.-M. Xu, A Compact Blast-Induced Traumatic Brain Injury Model in Mice, Journal of Neuropathology \& Experimental Neurology (2016) nlv019. [178] J.C. Mao, E. Pace, P. Pierozynski, Z. Kou, Y. Shen, P. VandeVord, E.M. Haacke, X. Zhang, J. Zhang, Blast-induced tinnitus and hearing loss in rats: behavioral and imaging assays, J Neurotrauma 29(2) (2012) 430-44.

[179] M.A. Gama Sosa, R. De Gasperi, P.L. Janssen, F.J. Yuk, P.C. Anazodo, P.E. Pricop, A.J. Paulino, B. Wicinski, M.C. Shaughness, E. Maudlin-Jeronimo, A.A. Hall, D.L. Dickstein, R.M. McCarron, M. Chavko, P.R. Hof, S.T. Ahlers, G.A. Elder, Selective vulnerability of the cerebral vasculature to blast injury in a rat model of mild traumatic brain injury, Acta Neuropathol Commun 2(1) (2014) 67. 


\title{
CHAPTER 3 ULTRASTRUCTURAL BRAIN
}

\author{
ABNORMALITIES AND ASSOCIATED BEHAVIORAL \\ CHANGES IN MICE AFTER LOW-INTENSITY BLAST
}

EXPOSURE

\subsection{ABSTRACT}

Explosive blast-induced mild traumatic brain injury (mTBI), a "signature wound" of recent military conflicts, commonly affects service members. While past blast injury studies have provided insights into TBI with moderate- to high-intensity explosions, the impact of primary low-intensity blast (LIB)-mediated pathobiology on neurological deficits requires further investigation. Our prior considerations of blast physics predicted ultrastructural injuries at nanoscale levels. Here, we provide quantitative data using a primary LIB injury murine model exposed to open field detonation of 350 grams of highenergy explosive $\mathrm{C} 4$. We quantified ultrastructural and behavioral changes up to 30 days post blast injury (DPI). The use of an open-field experimental blast generated a primary blast wave with a peak overpressure of 6.76 PSI $(46.6 \mathrm{kPa})$ at a 3 -m distance from the center of the explosion, a positive phase duration of approximate 3.0 milliseconds (ms), a maximal impulse of 8.7 PSI $x$ ms and a sharp rising time of $9 \times 10^{-3} \mathrm{~ms}$, with no apparent impact / acceleration in exposed animals. Neuropathologically, myelinated axonal damage was observed in blast-exposed groups at 7 DPI. Using transmission electron microscopy, we observed and quantified myelin sheath defects and mitochondrial abnormalities at 7 and 30 DPI. Inverse correlations between blast intensities and 
neurobehavioral outcomes including motor activities, anxiety levels, nesting behavior, spatial learning and memory occurred. These observations uncover unique ultrastructural brain abnormalities and associated behavioral changes due to primary blast injury and provide key insights into its pathogenesis and potential treatment.

\subsection{INTRODUCTION}

Blast exposure leading to traumatic brain injury (TBI) is recognized as a risk for subsequent cognitive and behavioral disorders as well as chronic neurodegenerative diseases $[1,2]$. During the conflicts in Iraq and Afghanistan, the focus on severe and penetrating brain injuries shifted to the more prevalent mild TBI (mTBI) [3, 4]. During 2000-2016, the Department of Defense's report (as of Jun 30, 2017) indicated that the vast majority (82.4\%) of TBI was classified as $\mathrm{mTBI} /$ concussion. Patients with an mTBI score 13-15 immediately post injury on the Glasgow Coma Scale (GCS), with the absence of abnormalities on computerized tomographic scanning [5]. Blast-induced mTBI may not be detectable using conventional imaging techniques, and immediate neurological signs and symptoms are usually transient while later chronic disorders occur in some individuals. Opportunities for early diagnosis and treatment are thus limited [3] and later development of post-traumatic stress disorder, other mental disorders or neurodegenerative diseases may result in lifelong disabilities $[1,6,7]$. The pathogenesis and prevalence of primary blast injury which may cause later chronic effects of mTBI remain controversial.

Experimental animal models using shock tubes have provided some valuable insights into blast injury mechanisms [8]. The underlying mechanisms of blast effects are 
based on the nature of the energy (physical force) transmitted and transduced across all spatial scales of brain tissue $[9,10]$. A primary blast wave (or overpressure), with an immediate and sharp change in pressure, travels through a medium (e.g., air) and interacts with the brain, mainly through the direct passage of the blast wave through the skull and the transfer of kinetic energy from the primary blast wave into the brain $[6,11]$. The blast wave induces sudden changes in intracranial pressure that result in shear stress, particularly at the interfaces between various cellular and subcellular structures within the brain [6]. Currently, only a few reports have used open-field explosions to study the blast injury [8, 12-14]. Open-field blasts generate a Friedlander waveform with a fast rise time and replicate the interactions between the primary blast overpressure and a shock wave reflection that bounces from the ground. The resulting amplification of the primary overpressure due to 'ground bounce' characterizes high-energy open-field explosions and is seen in our current model [15].

An understanding of blast physics is key to providing insight into injury mechanisms [16]. Among possible injury mechanisms, stress and strain in brain tissue may be caused by the direct cranial transmission of the blast energy or pressure wave $[10$, 17, 18]. In 2012, Kucherov et al. [17] proposed a novel phonon-based physical model to explain a potential cause of primary blast injury. The phonon is a quantum of energy or atomic vibration. Based upon water contents of the brain and cerebral spinal fluid, the Kucherov model predicted that blast-induced brain tissue damage would occur at nanoscale levels, in which subcellular injuries are expected to occur at the $30-200 \mathrm{~nm}$ range. Energy unloading takes place within microseconds of shock wave passage, in contrast to the milliseconds of impact acceleration injury [17]. 
Most blast-induced TBI studies have involved relatively high intensity blasts, resulting in a range of moderate to severe TBI with varying mortality rates [8]. The present study used open-field low intensity blast (LIB) to study behavioral effects associated with primary blast wave exposure. Behavioral and functional assessments, as well as neuropathological and transmission electron microscopy (TEM) methods were used to test the hypothesis that primary blast wave exposure could cause ultrastructural damage suggested by the prior physical modeling $[17,18]$. We observed ultrastructural brain damage and associated behavioral disorders resulting from primary LIB exposure in the absence of impact or acceleration. This report defines clear associations between fine structural alterations and behavioral disorders following LIB exposure. Insights gained may provide novel avenues of prevention and treatment toward subcellular brain injuries related to primary blast exposures.

\subsection{METHOD}

\subsubsection{Materials}

Chemicals were purchased from Sigma-Aldrich (St. Louis, MO, USA). Anesthesia:

Ketamine hydrochloride injection (100mg/mL, 1044102, Henry Schein, Melville, NY, USA), and Xylazine hydrochloride injection (100mg/mL, 13985-704-10, Vet One, Boise, ID, USA). FD NeuroSilver Kit II (Catalog\# PK301) was purchased from FD NeuroTechnologies (Ellicott City, MD, USA). Primary fixative (Electron Microscopy Sciences, Hartfield, PA, USA) for TEM contained 100 mM Sodium Cacodylate, 2\% glutaraldehyde, and 2\% paraformaldehyde. 


\subsubsection{Experimental design and procedure}

The animals were randomly grouped and blinded to investigators who carried out the experimental analyses. Mice in the 3-m and sham control groups were tested in shortterm (7 DPI) (n=14 for $3 \mathrm{~m}$ and $\mathrm{n}=12$ for sham) and long-term (30 DPI) $(\mathrm{n}=13$ for $3 \mathrm{~m}$ and $\mathrm{n}=12$ for sham), respectively. Mice were acclimated with the investigators for 5 days prior to the start of behavioral testing. Animals were then transferred to the Experimental Mine facility in the Rock Mechanics \& Explosives Research Center (RMERC) at Missouri University of Science \& Technology (Rolla, MO) 2 days before the blast, and transferred back to University of Missouri-Columbia animal facility (Columbia, MO) 2 days after the blast exposure. On the day of the blast experiment, mice were anesthetized with intraperitoneal (i.p.) injection approximately $5 \mu \mathrm{l} / \mathrm{g}$ body weight of Ketamine/Xylazine mixture ( $25 \mathrm{mg} / \mathrm{mL}$ ketamine and $1.25 \mathrm{mg} / \mathrm{mL}$ xylazine). Sham group underwent the identical procedures only without blast exposure. Mice were placed in the prone position at 3-, 4-, and 7-m platforms away from the $350 \mathrm{~g} \mathrm{C} 4$ (both $1 \mathrm{~m}$ above ground). No restrain was applied on the animals. Following blast wave exposure, animals were removed from the platform and returned to the original cage. After recovery from anesthesia, mice were able to spontaneously move and would continuously being monitored for at least 15-30 min.

After blast exposure, mice were tested in a variety of behavioral tasks: SNAP (Simple Neuroassessment of Asymmetric imPairment) $(\mathrm{n}=72)$, open-field $(\mathrm{n}=72)$, lightdark box ( $n=27)$, Y-maze $(n=72)$, nesting $(n=72)$, and Barnes maze $(n=27)$. These tasks assess motor activities, anxiety levels, spatial learning and memory in rodents. Animals were then euthanized either at 7 DPI or 30 DPI for anatomic and TEM studies. In sum, 
for short-term study, behavioral tests were performed in the following order: $\operatorname{SNAP}(1,3$, and 7 DPI), open-field (3 and 6 DPI), nesting (3-4 DPI), and Y-maze (7 DPI). For 30 DPI study, the behavioral tests were carried out in the following order: nesting (19-20 DPI), Y-maze (21 DPI), Barnes maze (22-29 DPI), SNAP (30 DPI) (Figure 3.1). Mice were acclimated to the testing room for $30 \mathrm{~min}$ prior to the start of each individual behavioral test. When more than one behavioral tests were performed within one day, each behavioral test was separated with a 2-h interval. All animals were allowed access to food and water ad libitum before and after blast exposure.

\subsubsection{Open-field LIB setting}

LIB exposures were conducted between $10 \mathrm{AM}-12 \mathrm{PM}$ during spring and fall seasons at the Rock Mechanics and Explosives Research Center at the Missouri University of Science \& Technology during moderate weather conditions. Environmental conditions were recorded for later potential replication. As previously reported, an experimental setting for open-field LIB was used [8]. Mice were placed in a prone position 3-m, 4-m, and 7-m distances away from the explosive in animal holders on a platform facing the explosive source with their head and body longitudinally oriented along the direction of

shock wave propagation. No restrain was applied on the animals. Sham group underwent identical procedures as blast groups only without blast exposure.

The blast quarry is an open space, with the dimension of $\sim 25 \times 35$ meters. The animal acclimating unit with temperature control and 12-hr timed lights in accordance with the animal welfare act has been installed away from the blast point which ensuring the animals in sham group were not affected by explosions. To ensure reproducible 
overpressures under LIB conditions for mTBI in mice, we used the following independent methods: 1) the blast UN SaferGuard Calculator was used to set the conditions; 2) pressure gauges were placed near the mice to measure pressure traces at a minimum sampling rate of $1 \mathrm{MHz}$ or greater; 3) test blasts with all equipment in place except for the mice were conducted and checked for accuracy and reproducibility, as we previously described [8].

High-speed videography was used to record the animal motion and C4 exposure dynamics during shockwave exposure. The digital video camera was placed $15 \mathrm{~m}$ from the blast focused on the animal's body, record times of 2000 frames per second captured animal motion. The video footage was recorded at 8000 frames per second, which was sufficient to capture both the primary peak and ground bounce. For high-speed videography analysis of the animal head motion during blast, we specifically pinpointed four coordinate points. Red points indicate the mouse's nose, left and right ear mark, respectively. The point in green on the platform was marked $50 \mathrm{~mm}$ away from the nose. Video and images were analyzed in blast real time from blast initiation to the end of the negative phase duration to ambient pressure.

\subsubsection{Modeling C4 blast waves}

The modeling of the C4 blast waves are described in more detail in D. Mott et al. 2008 [19], and are briefly summarized here. The composition of the C4 explosive is $90 \%$ RDX and $10 \%$ plasticizer. For these computations, the plasticizer is assumed to be inert, and the $\mathrm{C} 4$ decomposes into a gaseous mixture of carbon monoxide, carbon dioxide, solid carbon nanoparticles, nitrogen, and water. The initial conditions for the simulations are 
set after the $\mathrm{C} 4$ decomposes, assuming a constant volume reaction in the initial volume of the explosive. This sets the initial density, pressure, and temperature inside the explosive volume. The air outside the explosive is set at standard temperature and pressure $(298 \mathrm{~K}$ and 1.013 bar). The blast simulations are solved using the FBM-Blast code. This code is based on the multi-dimensional Flux-Corrected-Transport (FCT) algorithm developed at Naval Research Laboratory [20], and has been used extensively for blast mitigation studies [21], and to develop boundary conditions for helmet/blast wave interaction studies [19]. A cylindrical coordinate system is used so that the simulations can capture the reflection off the ground. This 'ground-bounce' is sensitive to the properties of the ground. Concrete surfaces are approximated as hard and perfectly reflective, which creates a stronger, faster wave reflection, whereas loose soil slows the reflection as dirt particles are lifted up and absorb some of the energy of the reflected wave. Calculations for a range of hardness demonstrated that the test results for the cases studies were most compatible with a modest effect of soil.

\subsubsection{Tissue processing, histopathology and immunohistochemistry}

At 7 or $30 \mathrm{DPI}$, mice were euthanized using isoflurane and brains were harvested as described previously [22]. Mice were euthanized at 7 and 30 DPI, transcardially perfused with 4\% paraformaldehyde (PFA) in $100 \mathrm{mM}$ phosphate buffer (PB) and their brains were dissected. Serial coronal sections were cut through the brains at $40 \mu \mathrm{m}$ thickness using a vibratome (VT1200S, Leica Microsystems, Inc., Bannockbum, IL, USA), and 120-150 tissue sections from each brain were sequentially collected and stereologically analyzed. 


\subsubsection{Hematoxylin and eosin $(H \& E)$ staining}

Gross brain tissue pathology was assessed using H\&E staining. Sections were mounted on the super frost slides before staining. Next, the sections were washed and incubated with different reagents for the following order: water (30 sec), hematoxylin 560 (2 min) (3801570, Surgipath ${ }^{\circledR}$ SelecTech ${ }^{\circledR}$, Leica Biosystems Richmond, IL, USA), water (1

min), Define (5 sec) (3803590), water (1 min), Blue buffer (30 sec) (3802915), water (45 $\mathrm{sec})$, eosin $(75 \mathrm{sec}), 95 \%$ ethanol ( $30 \mathrm{sec}), 100 \%$ ethanol $(60 \mathrm{~s}) 2$ times, xylene $(2 \mathrm{~min}) 2$ times. Then, coverslips were mounted with Fisher HealthCare ${ }^{\mathrm{TM}}$ PROTOCOL TM SecureMount $^{\mathrm{TM}}$ (022-208, Fisher Scientific, Pittsburgh, PA, USA).

\subsubsection{Silver staining and quantitative analyses}

The FD NeuroSilver Kit II was used for silver staining according to the manufacturer's instructions [23]. Each batch of the experiments was included the brain sections from different groups to avoid variability of the staining intensity.

For quantification of silver staining, serial images were stereologically captured of all sections using the Whole Slide Scanner (ScanScope CS-O, Aperio Technologies, Vista, CA) at the resolution of $0.25 \mu \mathrm{m}$ per raw image pixel (equivalent to $40 \mathrm{X}$ magnification), as previously reported [24]. The Spectrum Analysis algorithm package and ImageScope analysis software (version 11.0.2.725; Aperio Technologies, Inc.) were applied for quantitative analyses of various neurological events. For evaluation of neuronal degeneration in the silver stained tissue sections of the mouse brains, Color Deconvolution algorithm (Version 9.0) was used to separate an image into three channels 
corresponding to the actual colors of stains for quantification [25]. Tiff images of sections with the defined coordinates were captured containing the regions of interests of the CC\&EC (bregma 0.14 to $0.02 \mathrm{~mm}$ ), CP (bregma -1.94 to $-2.06 \mathrm{~mm}$ ), and fornix (bregma 0.14 to $0.02 \mathrm{~mm}$ ), based on the mouse brain atlas [26]. These images were exported and analyzed with ImageJ software (National Institutes of Health, Bethesda, MD).

Background (gold color) was subtracted from each image using the color splitter and image calculator functions, so that only the gray-black silver deposits were visible. Densitometric analysis of silver staining was then performed using ImageJ. Serial sections were averaged from each animal to produce a final silver staining value. The intensity of silver staining was expressed in arbitrary units, ranging from 0 (minimum) to 255 (maximum).

\subsubsection{Fluorescence immunohistochemistry and quantitative analyses}

Mouse brains were dissected and cut into serial coronal sections of 40-mm thickness by a vibratome (VT1200S, Leica Microsystems, Inc., Bannockbum, IL) after transcardiac perfusion fixation with $4 \%$ paraformaldehyde. Fixed coronal sections were permeabilized with $1 \%$ Triton $\mathrm{X}-100$ in phosphate-buffered saline (PBS) incubated with astrocyte marker (GFAP, 1:200, G9269; Sigma-Aldrich, St. Louis, MO) overnight. Then, the sections were incubated with fluorophore-conjugated secondary antibodies (1:200, chicken anti-rabbit IgG-Alexa488, A21441; Invitrogen, San Diego, CA), and then counter-stained with nuclear DNA dye Hoechst 33342 (1:1500). Fluorescence photomicrographs of the areas of interest were captured by a Leica DMI 6000B automated epifluorescence microscope (Leica Microsystems Inc., Buffalo Grove, IL). 
Stereological sections from bregma -1.94 to $-2.06 \mathrm{~mm}$, were used for quantification of GFAP expression levels in CC, CA1, dentate gyrus (DG), and CA3 regions. The region of interest was analyzed using ImageJ software. Sections from all mice in different groups were stained in a single batch. The mean percentage of the area for each region from each mouse was compiled for statistical analysis [27, 28].

\subsubsection{Transmission electron microscopy (TEM)}

\subsubsection{Tissue preparation}

Animals were anesthetized and perfused with saline $(0.9 \% \mathrm{NaCl})$ containing heparin $(20$ units $/ \mathrm{ml}$ ), and subsequently perfused with $4 \%$ paraformaldehyde. Total 13 animals were surveyed for TEM, including sham $(n=5), 3-\mathrm{m}$ blast animals euthanized at 7 DPI $(n=4)$, and 3-m blast mice euthanized at 30 DPI $(n=4)$. Brains were dissected and tissue sections were cut as $1-\mathrm{mm}$ thickness (approximately 6 coronal tissue blocks per brain) with the brain matrix (Ted Pella, Inc., Altadena, CA, USA), and post-fixed in primary fixative (Electron Microscopy Sciences, Hartfield, PA, USA) which contained 100 mM sodium cacodylate, $2 \%$ glutaraldehyde, and 2\% paraformaldehyde. Brain sections were further stereologically cut into $1 \mathrm{~mm}^{3}$ size from cerebral cortex (including corpus callosum), hippocampus, striatum, and cerebellum under light microscopy. Tissues were kept in 0.1 M NaCacodylate buffer $(\mathrm{pH}=7.4)$ with $0.13 \mathrm{M}$ sucrose all the time. Next, the tissues were embedded using 1\% osmium tetroxide (Ted Pella, Inc. Redding, California) in Cacodylate buffer. Finally, sections were cut to a thickness of $85 \mathrm{~nm}$ using an ultramicrotome (Ultracut UCT, Leica Microsystems, Germany) and a diamond knife (Diatome, Hatfield PA). Images were acquired with a JEOL JEM 1400 transmission 
electron microscope (JEOL, Peabody, MA) at $80 \mathrm{kV}$ on a Gatan Ultrascan $1000 \mathrm{CCD}$ (Gatan, Inc, Pleasanton, CA).

\subsubsection{Quantitative analysis}

The quantification was performed in a double-blinded fashion to the investigators and the TEM tissue sections of the brain were systematically and randomized surveyed in each region including cortex (layer III to V), striatum (regions including posterior thalamic nuclear group and ventral posteromedial thalamic nucleus), hippocampus (CA1) and CC (directly above the hippocampal CA1 area). We examined all myelin sheaths and mitochondria that were identified within each image field to determine ultrastructural abnormalities associated with blast injury. To quantify the myelin defects and mitochondrial abnormalities that showed clear and distinct hallmark evidences of damage, definitions of specific pathological changes are provided [29, 30]. On average, 30 to 40 non-overlapping microscopic fields $\left(10.70 \times 10.70 \mu \mathrm{m}^{2}\right)$ per region (cortex, hippocampus, and striatum) and 10-15 per CC were randomly captured at 2,000 X magnification for quantification, $\left(114.49 \mu \mathrm{m}^{2}\right.$ / image $)$ giving a total area of $15,456 \mu \mathrm{m}^{2}$ per animal examined, and 5,000 X or 10,000 X for illustrations and scrutiny of their distinct subcellular characteristics. This microscopic field allowed sampling and analyzing of approximately 1,900 axonal profiles and 2,800 mitochondria per animal.

\subsubsection{Myelin sheath defects}

Myelinated axons were distinguishable by the presence of the electron-dense myelin sheath. The observed myelin sheath defects were classified as: 1) redundant (excessive 
axonal myelination); 2) myelin balloon (bulges that are produced by splitting the myelin sheath layers); 3) myelin detachment (characterized by a hypodense peri-axonal space between the axon and myelin sheath); 4) myelin disruption (discontinuity of the myelin sheath) $[30,31]$.

\subsubsection{Mitochondrial abnormalities}

Mitochondria were classified into healthy (compacted matrix cristae, continuous outer membrane, regardless of size or shape) or damaged (fragmented cristae with evidence of an expanded matrix compartment or disrupted outer membrane) [32]. Mitochondrial abnormalities within the neuropil were then further analyzed and classified based on their ultrastructural appearance into one of following categories: 1) swollen clear mitochondrion (SCM) with swollen mitochondria with clear matrix; 2) disappearance of cristae and/or discontinuous inner and outer mitochondrial membranes; 3) swollen dense mitochondria (SDM) with dense matrix and fragmented cristae; and 4) dark degenerated mitochondria (DDM) with electron dense content with disorganized cristae and/or shrunken size [33].

\subsubsection{Behavioral assessment}

Behavioral tests after blast exposure were used to assess motor activities, anxiety levels, spatial learning and memory. We used SNAP, open-field, light-dark box, and nesting behavior tests for the short-term behavioral measurement; and Barnes maze tests for the long-term outcome assessment (Figure 3.1). 


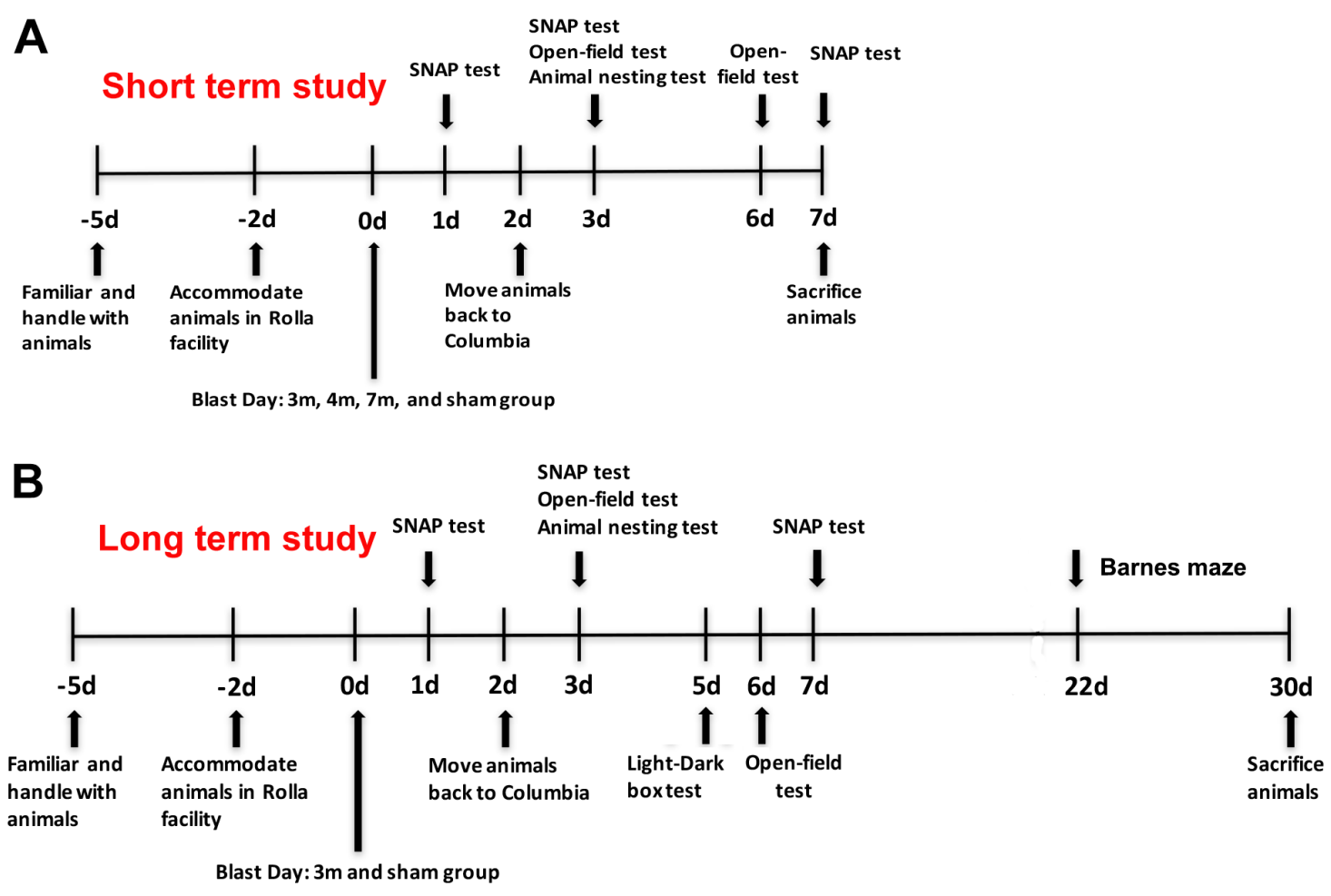

\section{Figure 3.1 Experimental Design.}

Schedules for blast experiments. Animals were euthanized either at 7 DPI (A) or 30 DPI (B). Behavioral tests performed at indicated intervals.

\subsubsection{SNAP}

The SNAP test was used to measure the neurological status and deficits induced in a mouse model of TBI. The tests examined interaction, cage grasp, visual placing, pacing/circling, gait/posture, head tilt, visual field, and baton as described previously [34]. SNAP provided a sensitive, reliable, time-efficient and cost-effective means of assessing neurological deficits in mice. Each individual test had a scoring range of 0-5 based on the guidelines. Our blast TBI animals yielded scores for each test of $0,0.5$, and 1 due to the mild nature of the blast effects globally affecting the brain. Although our 
open-field blast-induced global rather than unilateral injury on mice, SNAP test still served the purpose of evaluating neurological status and deficits.

\subsubsection{Open-field}

Locomotor/exploratory activity and anxiety-like behavior was tested in the open-field as we described previously [35]. The open-field activity box is $40 \times 40 \mathrm{~cm}$ and located on a white platform. The animals were placed into the center of the square arena for $10 \mathrm{~min}$. After each trial, the box was cleaned with $10 \%$ ethanol solution and air-dried. The animals' movements were tracked and analyzed using ANY-maze software (v.4.72, Stoelting Co., Wood Dale, IL; Windows XP OS). A series of the 10 x10-cm block were identified to evaluate subject's activity tracks with 4 blocks defined as the center zone and the surrounding 12 blocks as for the peripheral zone. Total distance traveled and time spent in different areas (center vs. periphery as a measure of anxiety) of the field were determined.

\subsubsection{Light-dark box}

The light-dark box is a behavioral measure of both novelty-seeking and anxiety-like behaviors in mice. It has the same area as the open-field test but half of the area is closed and a small opening $(7.5 \mathrm{~cm}$ high and $5 \mathrm{~cm}$ wide) can be found in the middle. The procedure is the same as for the open-field task [35]. Briefly, the animals are placed in the light side of the box and they were allowed to explore the box for $5 \mathrm{~min}$. Their movements were tracked and analyzed by ANY-maze. Three measures will be calculated 
for each mouse: 1) latency to first entry into the dark area, 2) number of entries to the dark area and 3) time spent in the light area.

\subsubsection{Nesting}

Nest building was tested by giving $5 \mathrm{~g}$ of cotton nestlet material as described previously [35]. Nests will be scored based on shape and unused materials [36]. Scores ranged from 1-5 using the following criteria for each score: 1 , nestlets not/barely touched; 2 , nestlets partially torn; 3 , nestlets mostly shredded, but no identifiable nesting site (i.e., material is distributed throughout the cage); 4 , nestlets almost entirely shredded and gathered into one-quarter of the cage, but the nest is flat; and 5, nestlets almost entirely shredded, gathered into one-quarter of the cage; nearly perfect nest, crater-shaped, with walls higher than the mouse's body height. Any unshredded nesting material was weighed and recorded for each mouse.

\subsubsection{Barnes maze}

Spatial learning and memory was tested in the Barnes maze as described before [22]. The maze consists of a circular platform ( $75 \mathrm{~cm}$ diameter) with 20 holes $(5 \mathrm{~cm}$ diameter) evenly spaced around the platform's perimeter; it is elevated $56.5 \mathrm{~cm}$ above the floor by a stand. For each mouse, one hole was assigned as the escape hole, and an escape box was located beneath the hole. The escape box location remained constant for any individual mouse across test trials. Three $100-\mathrm{W}$ lights were hung above the platform to create a potentially adverse environment and motivate the mice to escape from the brightly lit, open surface (in favor of the dark environment of the box). Behavioral testing consisted 
of 2 shaping trials on the first day, and 8 evaluation trials ( 2 trials/day) over the next 4 days. Reversal training consisted of 2 trials per day for 3 days, with an inter-trial interval of 20-30 min and began $24 \mathrm{~h}$ after the last acquisition trial. Prior to the start of reversal training, each mouse is assigned a new escape hole, which is on the opposite side of the maze from the previously assigned hole. Latency (time it took for the mouse to find the escape box) and total errors (nose-pokes into non-escape holes) were recorded and analyzed by ANY-maze.

\subsubsection{Data and statistical analysis}

Statistical analyses were performed using Prism (GraphPad Software). Blast peak overpressure, positive phase duration and weather data are presented as Mean \pm Standard Deviation (SD). The following pathological analysis and behavioral test data are presented as Mean \pm Standard Error of Mean (SEM). One-Way ANOVA was used to compare differences among blast and sham groups in body weight measurement; openfield, light-dark box, and nesting behavior analyses; as well as silver staining measurement and TEM quantification. Two-way ANOVA was used to analyze SNAP, open-field and Barnes maze data. $P$ values of post hoc tests for multiple comparisons were adjusted using the Tukey test. Bonferroni test was used to adjust for multiple comparisons specifically for Barnes maze test. Unpaired t-test was applied for comparison between any two groups. Differences were considered significant at $P<0.05$ for all analyses, with * indicating $P<0.05$; **, $P<0.01$; ***,$P<0.001$; and ****, $P<0.0001$. 


\subsection{RESULTS}

\subsubsection{Characterization of LIB exposure}

Pressure time histories confirmed experimental approximation of the Friedlander waveform of the primary blast wave overpressure. Peak overpressure measurements were uniformly reproducible, with values of $6.76 \pm 0.50$ PSI $(\sim 46.6 \mathrm{kPa})(\mathrm{n}=26), 4.62 \pm 0.38$ PSI $(\sim 31.9 \mathrm{kPa})(\mathrm{n}=6)$, and 2.84 \pm 0.10 PSI $(\sim 19.6 \mathrm{kPa})(\mathrm{n}=8)$ for $3-\mathrm{m}, 4-\mathrm{m}$, and $7-\mathrm{m}$ distances away from blast, respectively. Positive phase durations were $2.82 \pm 0.15 \mathrm{~ms}$, $3.08 \pm 0.12 \mathrm{~ms}$, and $3.56 \pm 0.15 \mathrm{~ms}$ for the three groups correspondingly (Table 3.1 , Figure 3.2A, and Figure 3.3).

Table 3.1 Data for primary peak overpressure, positive phase duration and impulse of LIB recorded from the pencil gauges mounted on each platform.

\begin{tabular}{|l|l|l|l|l|l|l|l|}
\hline \multicolumn{2}{|l|}{ Placement of the pencil gauges } & $\begin{array}{l}3 \mathrm{~m} \\
\text { (right) }\end{array}$ & $\begin{array}{l}3 \mathrm{~m} \\
(\mathrm{left})\end{array}$ & $\begin{array}{l}4 \mathrm{~m} \\
\text { (right) }\end{array}$ & $\begin{array}{l}4 \mathrm{~m} \\
\text { (left) }\end{array}$ & $\begin{array}{l}7 \mathrm{~m} \\
\text { (right) }\end{array}$ & $\begin{array}{l}7 \mathrm{~m} \\
\text { (left) }\end{array}$ \\
\hline \multirow{2}{*}{$\begin{array}{l}\text { Primary peak } \\
\text { overpressure (PSI) }\end{array}$} & Average & 6.73 & 6.78 & 4.69 & 4.55 & 2.82 & 2.87 \\
\cline { 2 - 9 } & SD & 0.51 & 0.51 & 0.36 & 0.46 & 0.10 & 0.12 \\
\hline $\begin{array}{l}\text { Overall positive phase } \\
\text { duration (ms) }\end{array}$ & Average & 2.86 & 2.78 & 3.06 & 3.11 & 3.54 & 3.59 \\
\cline { 2 - 9 } & SD & 0.15 & 0.14 & 0.14 & 0.12 & 0.04 & 0.22 \\
\hline $\begin{array}{l}\text { Maximum Impulse } \\
\text { (psi*ms) }\end{array}$ & Average & 8.70 & 8.67 & 6.99 & 6.71 & 4.27 & 4.28 \\
\cline { 2 - 9 } & SD & 0.31 & 0.36 & 0.12 & 0.15 & 0.12 & 0.25 \\
\hline
\end{tabular}



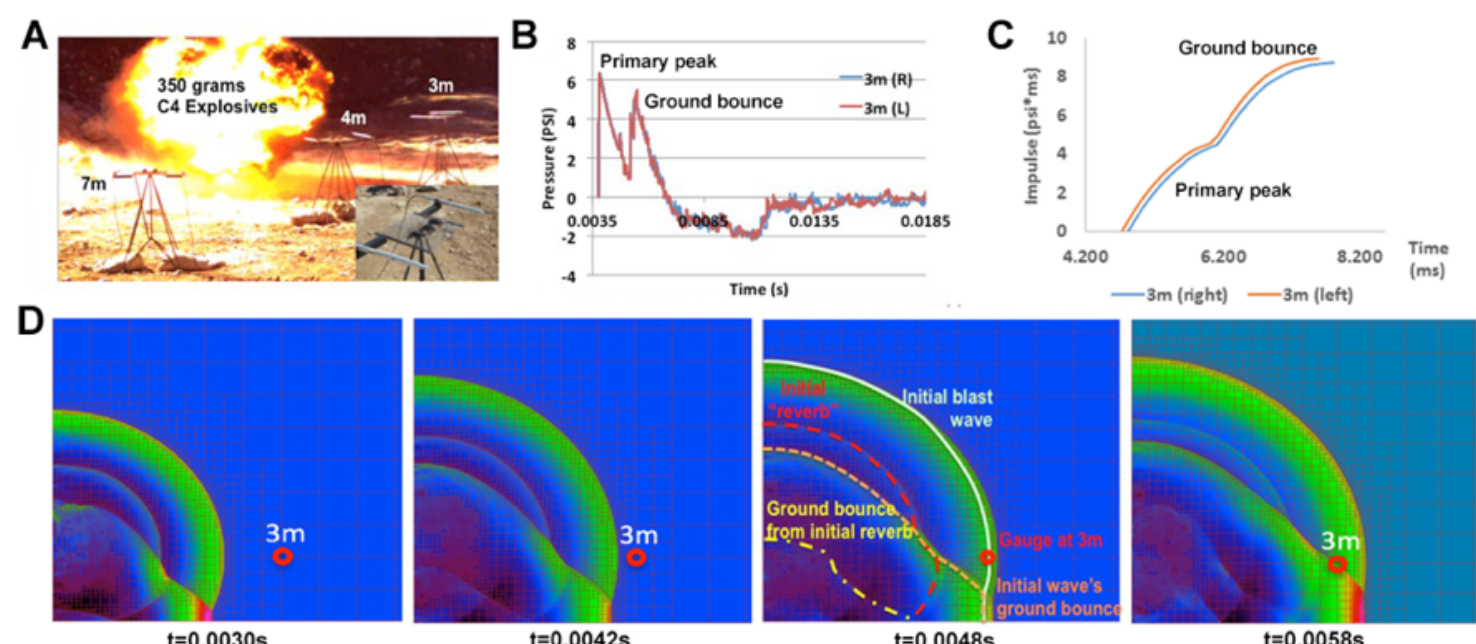

Figure 3.2 Open-field blast setting, characterization and kinetics.

Open-field blast settings to induce primary blast injury in mice, blast pressure traces, and shock wave simulations. (A) Representation of the blast onset captured by a Blaster's Ranger II 2500-fps high-speed video camera. Blast with $350 \mathrm{~g}$ of C4 explosive at standoff distances: 3-, 4-, and 7-m. Inset: the enlarged mouse holding platform and placement of two pencil pressure gauges (137B23B - ICP blast pressure probe, $50 \mathrm{psi}, 100 \mathrm{mV} / \mathrm{psi}$; PCB Piezotronics) on each platform. (B) Peak pressure vs. time for left/right (L/R) transducers placed at the $3 \mathrm{~m}(\mathrm{n}=26), 4 \mathrm{~m}(\mathrm{n}=6)$, and $7 \mathrm{~m}(\mathrm{n}=8)$ platform. (C) Time vs. impulse curves for two transducers at distances of 3-, 4-, and 7-, m. (D) Simulation of the blast shock waves based on the recordings of the pencil pressure gauges at the 3-m distance. The shockwave structure passing the 3-m platform location is relatively complex, and modest changes in the modeling or geometry can affect the magnitude and structure of the second ('ground bounce') peak. 

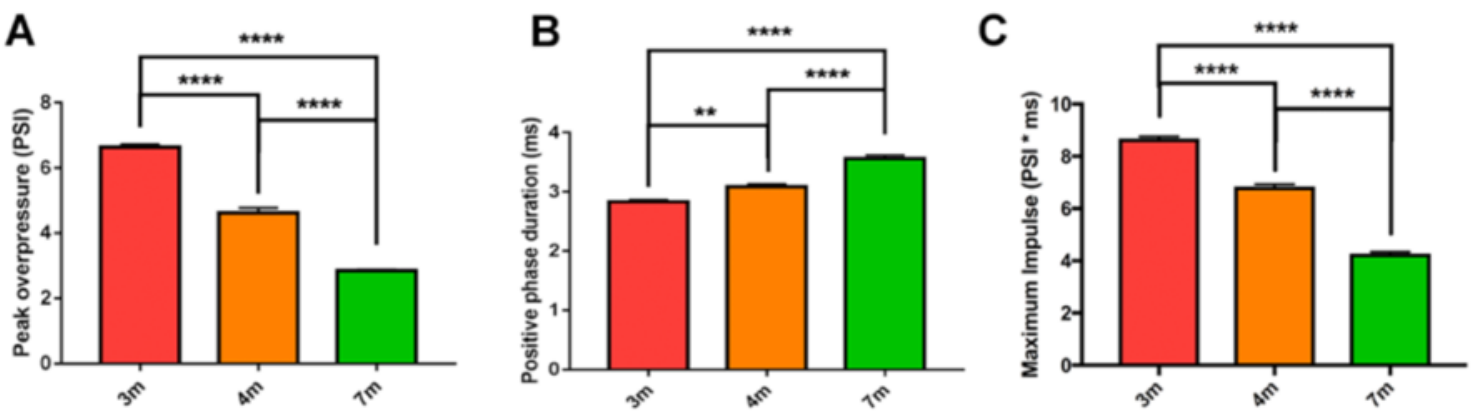

Figure 3.3 Blast overpressures, positive phase durations, and maximum impulses changed at varying blast distances.

(A) Comparison of blast peak overpressure among 3-m, 4-m, and 7-m blast groups. Oneway ANOVA: $P<0.0001$. (B) Comparison of blast positive phase duration among 3-m, 4m, and 7-m groups. (One-way ANOVA: $P<0.0001)(\mathrm{C})$ Comparison of blast maximum impulse among 3-m, 4-m, and 7-m groups. One-way ANOVA: $P<0.0001 ; \mathrm{n}=26$ for 3-m, $\mathrm{n}=6$ for $4-\mathrm{m}$, and $\mathrm{n}=8$ for $7-\mathrm{m}$ blast groups.

In addition to peak overpressures and short positive phase duration closely mimicking the ideal Friedlander wave, the recording of the pressure sensors showed a sharp rising time (duration of ambient pressure reached highest overpressure) within 6-9 $\mathrm{x} 10^{-3} \mathrm{~ms}$ (Figure 3.2B). The dynamic blast impulses (Table 3.1 and Figure 3.2C) revealed a greater positive phase at $3-\mathrm{m}$ (maximum impulse $8.69 \pm 0.34$ PSI x ms) as compared to 4-m and 7-m distances, as related to differentiating features of the blast 'primary peak' and 'ground bounce'. Pressure traces indicated that the secondary peak 'ground bounce' sustained both longer overpressure and enhanced impulse of blast exposures. Computational analyses revealed blast interactions with complex geometries based on recordings from the pencil pressure gauges on each animal platform (Figure 
3.2D). The computed arrival time and peak pressure of the primary blast wave (illustrated in green) at the 3-m sensor (red circle) agreed well with experimental data. The secondary peak ('ground bounce') at 3-m corresponded with the third image from left in Figure 1B and appeared to be a combination of the initial wave's 'ground bounce' along with spherical explosive's initial reverberation. These analyses provide novel insights into the blast forces and their mechanisms underlying open-field primary LIB exposures.

Table 3.2 Data of ambient environmental conditions for the LIB exposures.

\begin{tabular}{|l|l|l|}
\hline Weather Information & Average & SD \\
\hline Temperature ( $\left.{ }^{\circ} \mathrm{F}\right)$ & 63.25 & 8.65 \\
\hline Humidity (\%) & 69.00 & 15.21 \\
\hline Wind speed (mph) & 10.00 & 5.37 \\
\hline
\end{tabular}



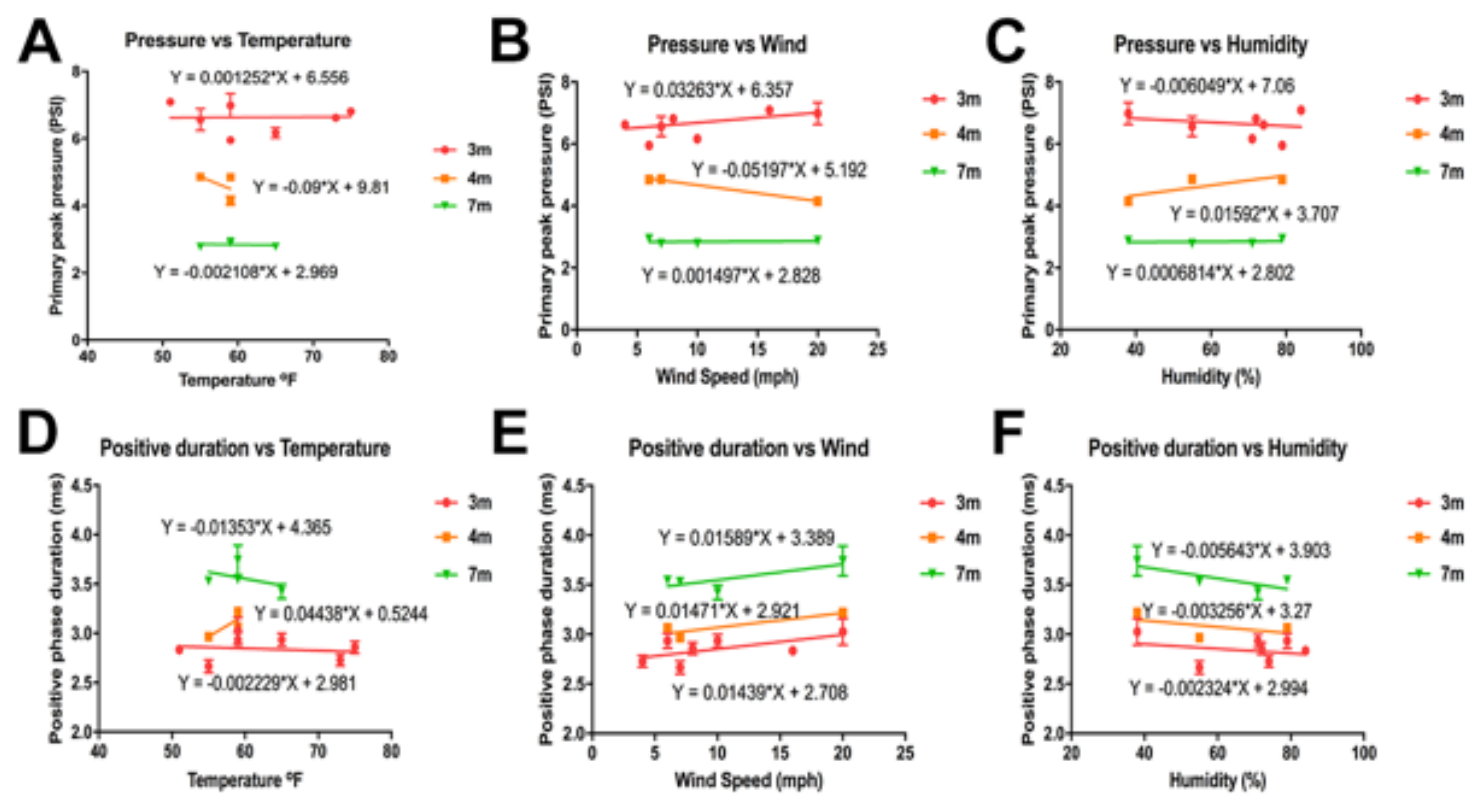

Figure 3.4 Weather conditions of the ambient environment in the open-field blast quarry did not affect blast conditions.

Correlation of weather information (temperature, wind speed, and humidity) with blast physical parameters including primary peak overpressure and positive phase duration were shown in A to $\mathrm{F}$ ( $\mathrm{n}=22$ for $3-\mathrm{m}, \mathrm{n}=6$ for $4-\mathrm{m}$, and $\mathrm{n}=8$ for $7-\mathrm{m}$ blast groups), independently. Individual linear regression equation was shown near the specific regression line. The blast physical parameters were not significantly affected by weather conditions.

Using current experimental procedures and timing, ambient weather conditions, including temperature $\left(63.25 \pm 8.65^{\circ} \mathrm{F}\right)$, humidity $(69.00 \pm 15.21 \%)$, and wind speed $(10.00 \pm 5.37 \mathrm{mph})$, did not cause variations in blast peak overpressure and positive phase duration (Table 3.2 and Figure 3.4). Using high-speed videography with the analysis of the coordinates among the anatomic marks, no apparent animal head or torso 
displacements were seen to occur during exposure to the primary blast wave (Figure 3.5).

These findings indicate that behavioral deficits and subcellular damage resulting from the LIB exposures were clearly related to a primary blast wave injury affecting brain tissue and its subsequent effects rather than by impact or accelerative forces. As previously described, there was no mortality or lung injury using this open-field LIB model [8]. Blast exposed animals recovered from anesthesia similar to sham controls.

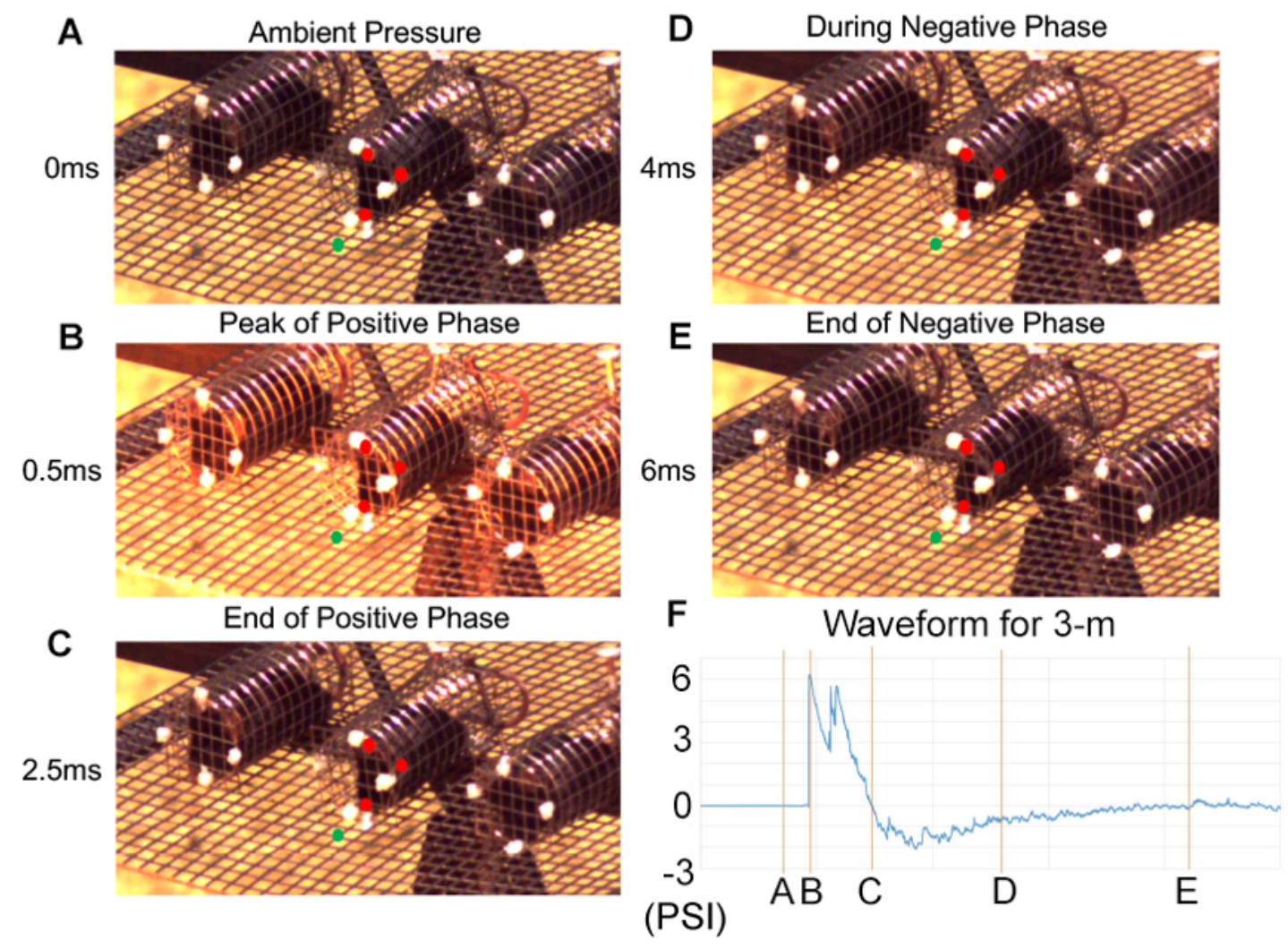

Figure 3.5 Serial images from high-speed video confirm no apparent head movement of the mice exposed to low-intensity open-field primary blast.

We pinpointed four coordinate points. Red points indicate the mouse's nose, left and right ear mark, respectively. The point in green on the platform was marked $50 \mathrm{~mm}$ away from the nose. There was no apparent change in the coordinates of nose point and green 
reference point during the primary blast wave pass through the mice. Representative images were shown in real time $0 \mathrm{~ms}$ (ambient pressure at blast initiation) (A), $0.5 \mathrm{~ms}$ (at the peak of positive phase) (B), $2.5 \mathrm{~ms}$ (at the end of positive phase) (C), $4 \mathrm{~ms}$ (during the negative phase) (D), and $6 \mathrm{~ms}$ (at the end of negative phase back to ambient pressure) (E), with corresponding waveform respectively (F). The evidence indicates there is no acceleration. Additionally, there was no apparent change in the coordinates of the two ear points and green reference point, indicating that no apparent head movement occurred during blast exposure.

\subsubsection{Histopathology and immunohistochemistry of blast injuries}

\subsubsection{Brain H\&E and GFAP staining}

As previously described [8], no gross or light microscopic changes occurred with a peak overpressure of equal or less than 6.76 PSI (46.6 kPa). Hematoxylin and eosin (H\&E) staining revealed no macroscopic damage or necrosis in brain tissue in 3-, 4- and 7-m blast groups at 7 DPI and 30 DPI (Figure 3.6). Glial responses appeared to be absent. To further investigate apparent paucity of astroglial responses at these exposure levels, we measured glial fibrillary acidic protein (GFAP) expression for astrocytes within different brain sub-regions (Figure 3.7). No significant differences in GFAP in corpus callosum (CC), hippocampal CA1, dentate gyrus (DG), and hippocampal CA3 brain regions between the 3-m and the sham control were seen. 


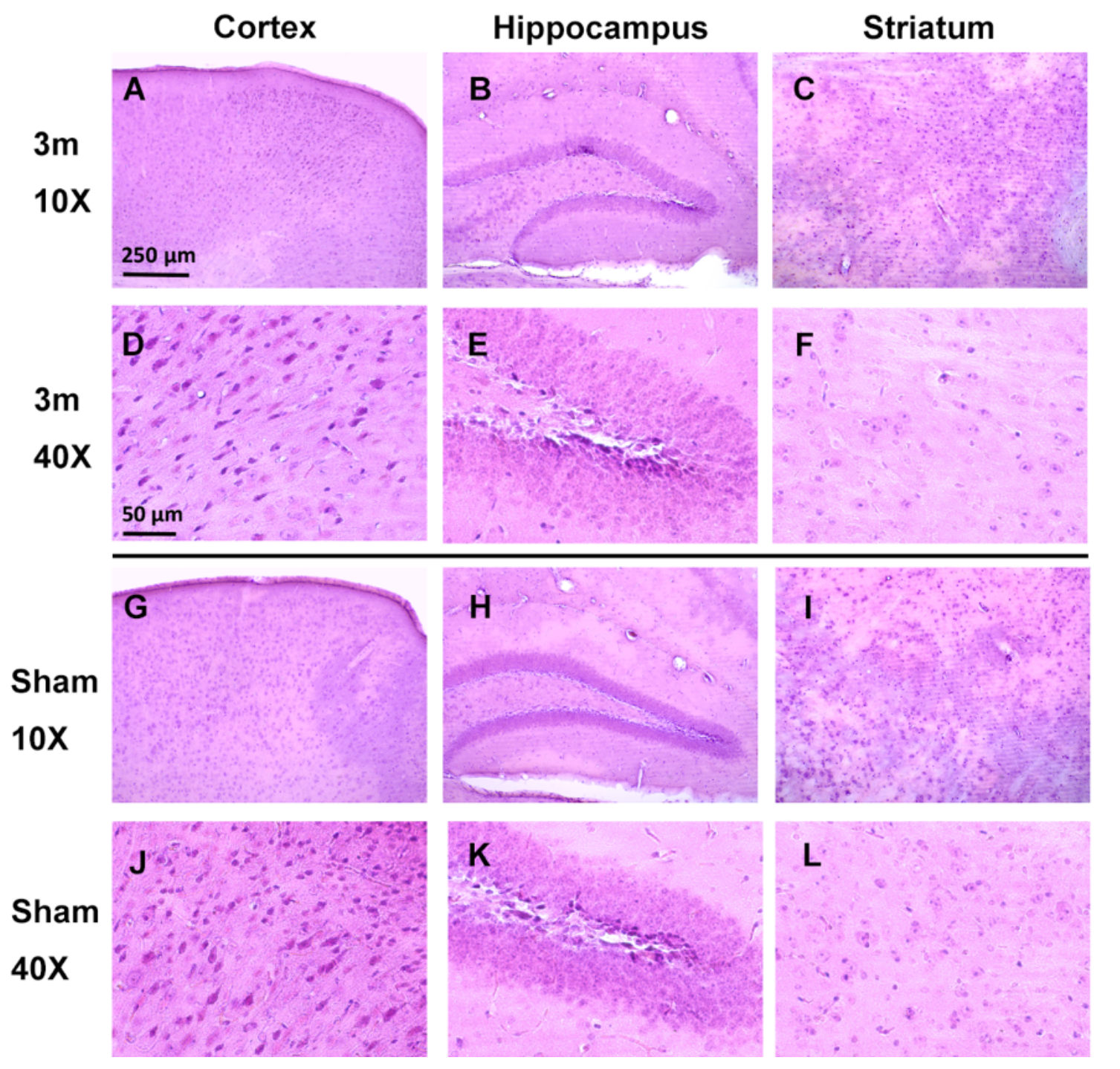

Figure 3.6 Hematoxylin \& Eosin (H\&E) staining for brain tissue in 3-m blast and sham group.

Representative images of histopathological specimens of brain tissues stained with H\&E method. (A-F) 3-m blast group at 7 DPI under $10 \mathrm{X}$ and $40 \mathrm{X}$. (G-L) Sham group at 7 DPI under $10 \mathrm{X}$ and $40 \mathrm{X}$. No obvious macroscopic damage or necrosis was observed among different brain sub-regions in the blast group compared to sham controls; $n=3$ in each group. 


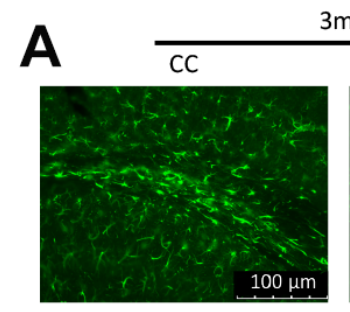

DG

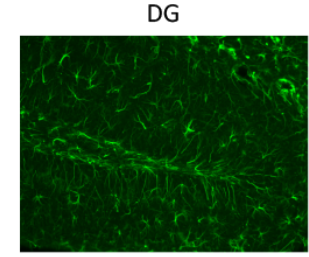

B

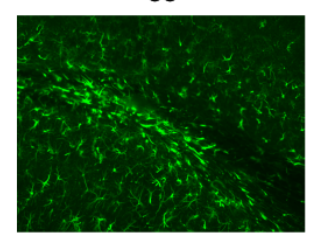

DG

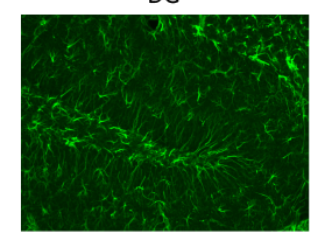

$3 \mathrm{~m}$

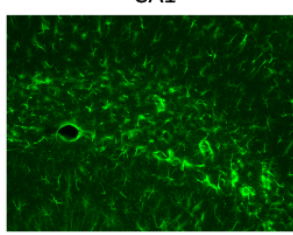

CA3

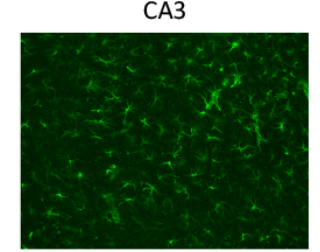

CA1

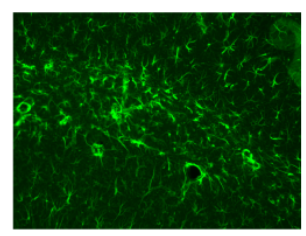

CA3

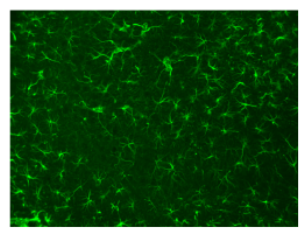

C

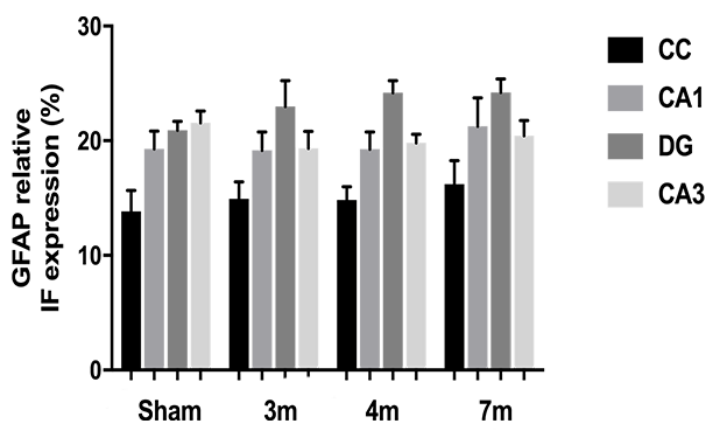

D

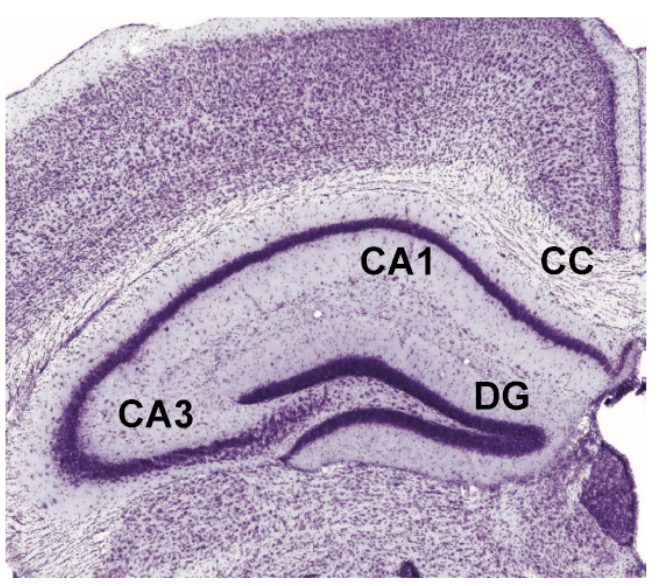

Figure 3.7 GFAP expression levels not significantly altered after LIB exposure.

Representative images were shown as left hemisphere of the brain. (A and B) GFAP staining were performed and quantified in $\mathrm{CC}, \mathrm{CA} 1, \mathrm{DG}$, and $\mathrm{CA} 3$ regions of the brain in 3-, 4-, and 7-m and sham group. GFAP staining was not obvious in other brain subregions. (C) Quantification analysis showed no significant difference in GFAP relative expression level between the two groups in all four regions. (D) Brain sections (CC, CA1, DG, and CA3) were chosen based on mouse-brain atlas, as shown. Sections at Bregma -1.94 mm to $-2.06 \mathrm{~mm}$ were used. Two-way ANOVA: P $>0.05$; Tukey test for all comparisons in the same brain sub-regions between sham and blast groups: $\mathrm{P}>0.05 ; \mathrm{n}=3$ in each group. 


\subsubsection{Axonal injury after LIB exposure}

Axonal injury in LIB-exposed animals occurred in the absence of macroscopic cell body damage. Degenerating axon-terminals and dendrites, with high affinity for silver (argyrophilia) were visualized on silver-stained tissue sections (Figure 3.8).

Densitometric analyses by NIH ImageJ were performed for regions of corpus callosum \& entorhinal cortex (CC\&EC) (which reflect the axonal bundles connecting the cerebral hemispheres to facilitate interhemispheric communications), cerebral peduncle (CP) (which contains the large ascending and descending nerve tracts that run between cerebellum and pons), and fornix (which carries signals from the hippocampus to the thalamic nuclei). The silver stain intensities in 3-, 4- and 7-m blast groups were significantly higher than in the sham group at 7 DPI (Figure 3.8). The abnormalities at 7 DPI appeared to return to the sham control comparable levels at 30 DPI (data not shown). To examine the effects of blast wave transmission through the brain tissue, we compared the silver staining intensities of rostral and caudal cortical tissues in 3-m blast at 7 DPI. The silver staining intensities were higher in rostral as compared to caudal in 3-m blast group. These findings suggest reduction of energy unloading from front to back during the transmission of the primary blast wave with greater damage to frontal brain areas. 


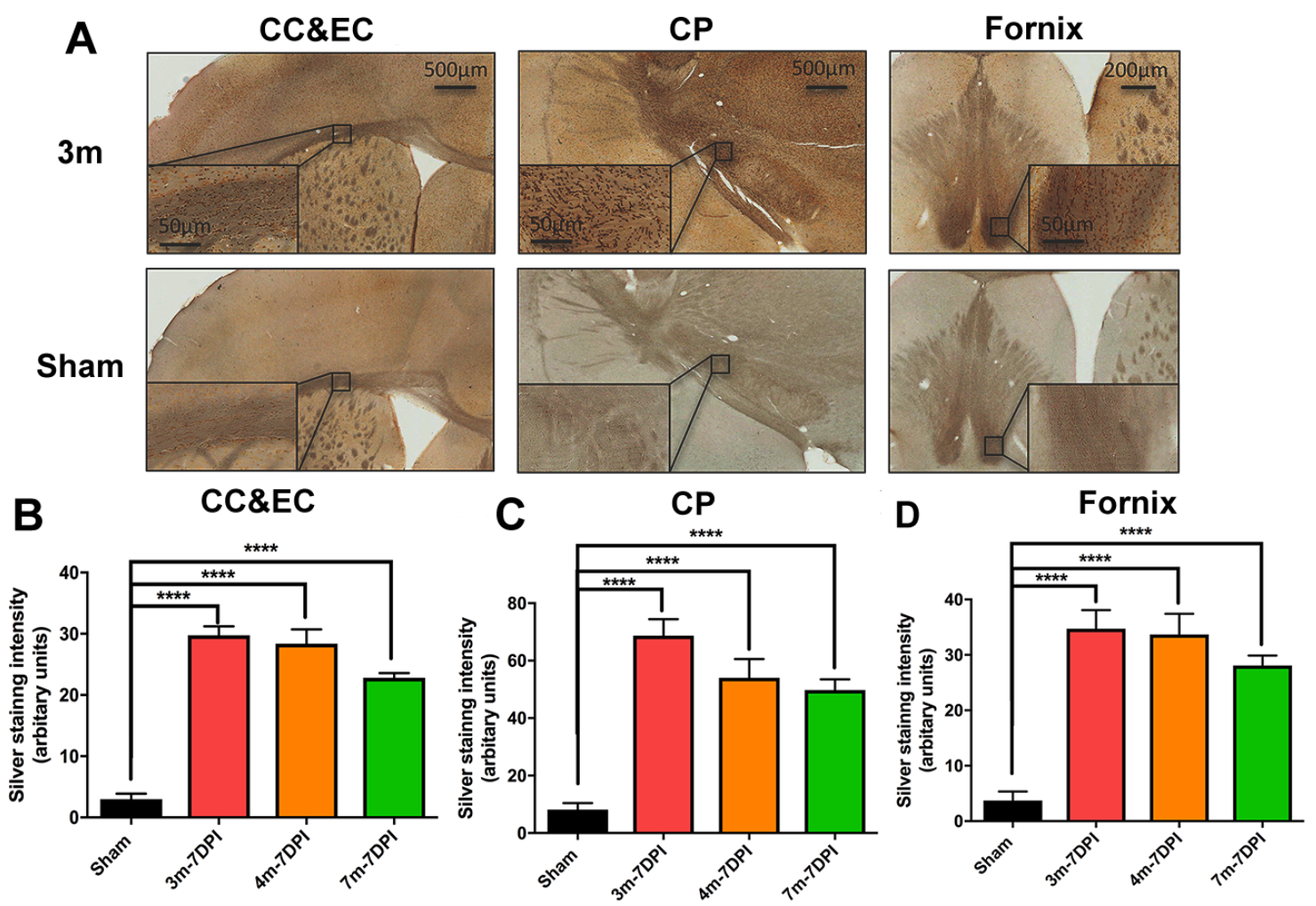

Figure 3.8 Axonal injury detected and quantified after LIB exposure by silver staining.

Silver staining measurement in primary LIB injury. (A) Representative images from the 3-m blast and sham groups showed axonal injury in the regions of CC \& EC, CP, and fornix. The inset boxes represent the higher magnification of the indicated area. (B-D) Statistical analysis. Silver staining was expressed ranging from 0 to 255 in arbitrary units. (B) Intensity of silver staining in CC\&EC region in 3-, 4-, and 7-m blast groups at 7 DPI were significantly higher than that in the sham group. One-way ANOVA: $\mathrm{P}<0.0001$, multiple comparisons (Tukey); sham vs. 3-, 4- and 7-m at 7 DPI, $\mathrm{P}<0.0001$; 3-m at 7 DPI vs. 7-m at 7 DPI, $\mathrm{P}=0.0082 ; \mathrm{n}=6$ for $3-, 4$ - and $7-\mathrm{m}$ at 7 DPI; $\mathrm{n}=11$ for sham. (C) Intensity of silver staining in CP region in 3-, 4- and 7-m blast groups at 7 DPI were significantly higher than that in the sham group. One-way ANOVA: P $<0.0001$, multiple 
comparisons (Tukey); sham vs. 3-, 4- and 7-m at 7 DPI, P $<0.0001$; 3-m at 7 DPI vs. 7-m at $7 \mathrm{DPI}, \mathrm{P}=0.028$. (D) Intensity of silver staining in fornix region in 3-, 4- and 7-m blast

groups at 7 DPI were significantly higher than that in the sham group. One-way ANOVA, $\mathrm{P}<0.0001$; multiple comparisons (Tukey); sham vs. 3-, 4- and 7-m blast at 7 DPI, $\mathrm{P}<0.0001$.

\subsubsection{Ultrastructural identifications of myelin defects and mitochondrial}

\section{abnormalities}

Based upon prior physical modeling of blast effects on water suggesting that mild blastinduced brain tissue damage would occur at nanoscale injury intervals [17], we used TEM to examine ultrastructural blast effects. Normal myelin sheaths in sham control animals were observed in the $\mathrm{CC}$, known for its high number of myelinated axonal bundles. These appeared as highly electron-dense formations with continuous and tightly wrapped myelin sheet around the axonal cytoplasmic membrane (Figure 3.9A-B). The 3$\mathrm{m}$ LIB exposed animals exhibited significantly higher numbers of myelin defects at 7 DPI (Figure 3.9H). Myelin defects appeared as extensive split layers (Figure 3.9C), dense degeneration (pockets of dense cytoplasm and vacuole within the myelin layers) (Figure 3.9D), myelin ballooning (bulges produced by splits in the myelin sheath layers) (Figure 3.9E), myelin disruption (rupture of the myelin continuity) (Figure 3.9F), or myelin detachment (hypodense peri-axonal space between the axon and myelin sheath) (Figure $3.9 \mathrm{G})$. These appearances returned to those of sham controls at $30 \mathrm{DPI}$. 

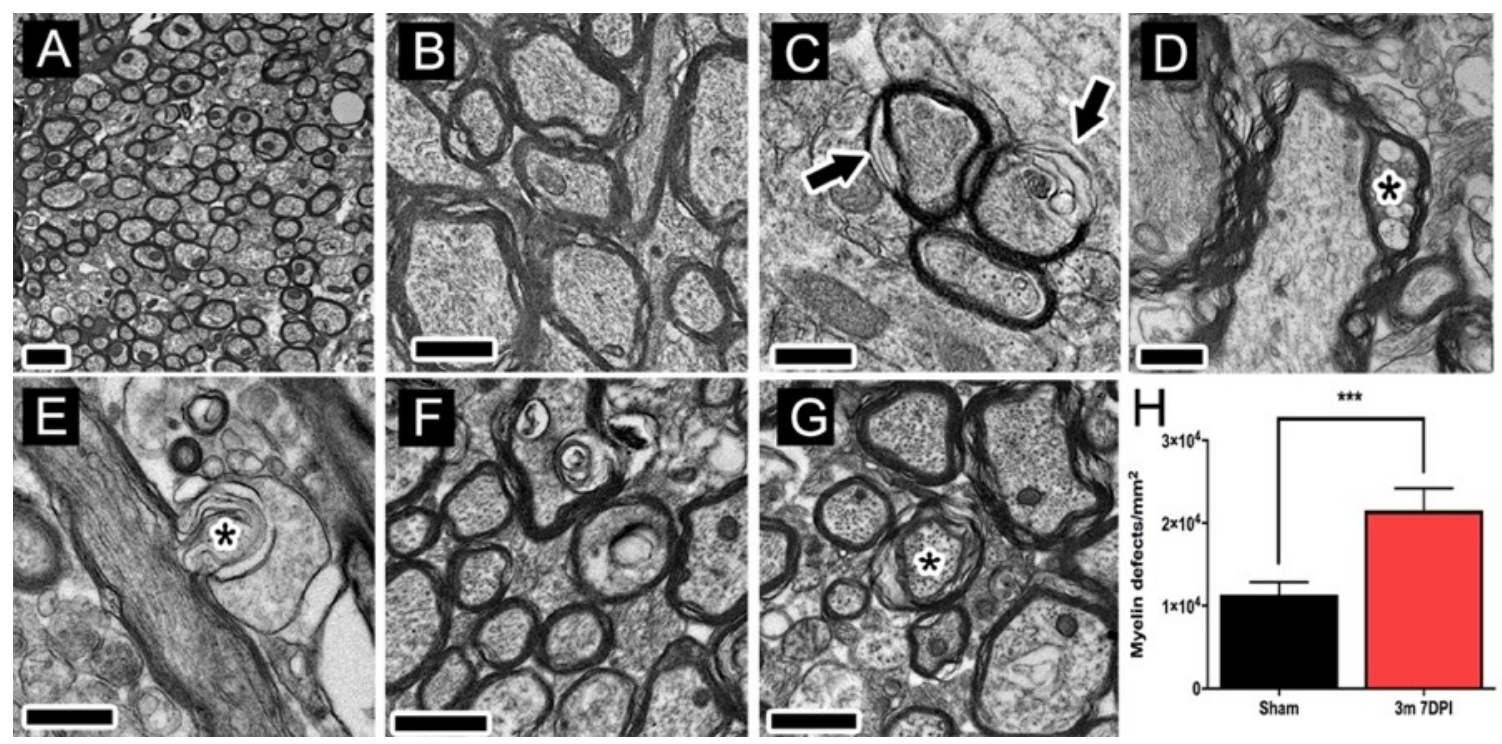

Figure 3.9 Myelin sheath defects identified in the corpus callosum after LIB

exposure.

TEM ultrastructural identification of myelin defects. (A) Representative image of low magnification $(2000 \times)$ field of CC region from sham animal (scale bar $=1 \mu \mathrm{m}) .(\mathrm{B})$

Normal myelin sheaths in sham group are thick, electron-dense and tightly wrap the axon (scale bar $=1 \mu \mathrm{m}) .(\mathrm{C}-\mathrm{G})$ Myelin sheath defects after 3-m blast injury at 7 DPI. (C) Myelinated axons with extended area of "disrupted" split myelin layers are shown by the arrows (scale bar $=1 \mu \mathrm{m}$ ). (D) Dense "redundant" myelin sheath degeneration represented by pockets of vacuoles and dense cytoplasm within the myelin layers indicated by the asterisk (scale bar $=0.5 \mu \mathrm{m}$ ). (E) "Myelin balloon" is characterized by bulges of split myelin layers indicated by the asterisk (scale bar $=0.5 \mu \mathrm{m}$ ). (F) Myelin disruption is an area of myelin sheath discontinuity around the axons (scale bar $=1 \mu \mathrm{m}$ ). (G) Myelin "detachment" is loosely wrapped abnormal myelin sheaths leaving a hypodense space between the axon membrane and the inner myelin layers indicated by the asterisk (scale bar $=1 \mu \mathrm{m}) .(\mathrm{H})$ Comparisons of the myelin sheath defects among 
sham and 3-m blast at 7 DPI. A significantly higher number of myelin sheath defects were observed in 3-m blast at 7 DPI as compared to sham. One tailed paired $t$ test, $\mathrm{P}=0.0005 ; \mathrm{n}=5$ for sham; $\mathrm{n}=4$ for 3 -m blast at 7 DPI.

Striking mitochondrial damage occurred in 3-m LIB exposed animals. Healthy mitochondria in sham control animals appeared on TEM as round / ovoid shape structures with double membranes, parallel-organized cristae and dense matrix (Figure 3.10A-B). After LIB exposure, we observed extensive mitochondrial abnormalities within the neuropil characterized as swollen clear (Figure 3.10C), swollen dense (Figure 3.10D), and dark degenerated mitochondria (Figure 3.10E). After 3-m blast animals both at 7 DPI and 30 DPI, significant higher numbers of mitochondrial abnormalities were persistently observed in cortex, hippocampus, and striatum (Figure 3.10F-H). 


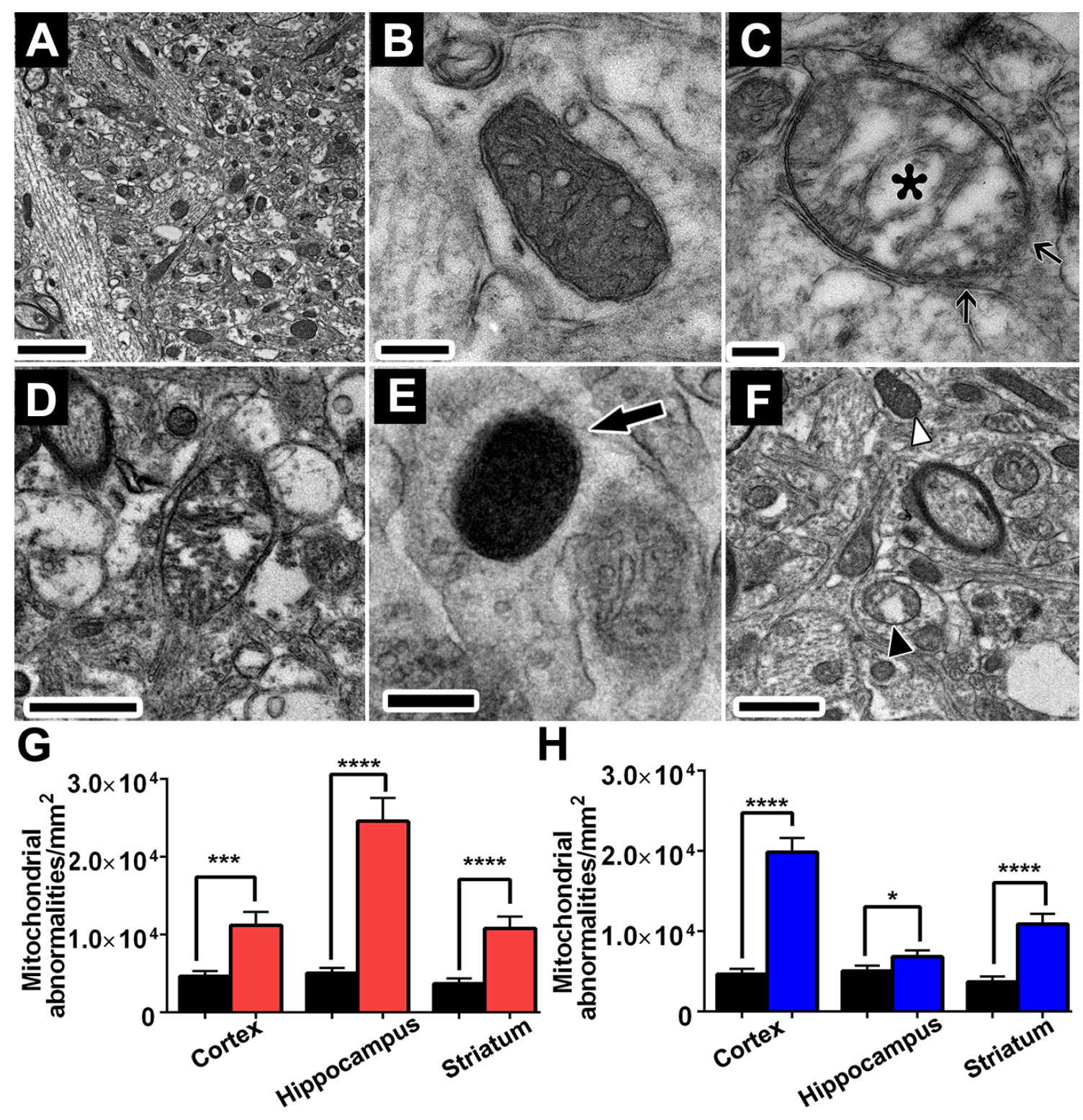

Figure 3.10 Mitochondrial abnormalities identified in the cortex, hippocampus, and striatum of the brain after LIB exposure both at 7 DPI and 30 DPI.

TEM ultrastructural identifications of mitochondria abnormalities. (A) Representative image of low magnification $(2000 \times)$ image of mitochondrial population from sham animal (scale bar $=1 \mu \mathrm{m}$ ). (B) Normal mitochondrion in sham group exhibited double membrane integrity, homogenous dense matrix and organized cristae (scale bar $=0.2 \mu \mathrm{m})$. (C) SCM, as indicated by asterisk, was observed after 3-m blast shown the 
destruction of the internal cristae leading to an electron lucent appearance of the matrix, arrows indicate the rupture of the double membrane (scale bar $=0.2 \mu \mathrm{m}$ ). (D) SDM, which exhibited multiple electron-lucent spots within the matrix corresponding to the destruction of the cristae, was shown after 3-m blast. (scale bar $=1 \mu \mathrm{m}$ ). (E) DDM, as recognized by its double membrane with an electron hyper dense collapsed matrix, was observed after $3-\mathrm{m}$ blast (scale bar $=0.2 \mu \mathrm{m})$. $(\mathrm{F})$ Representative image field with coexistence of normal mitochondria (white triangle) and abnormal mitochondrion (black arrow) after 3-m blast injury (scale bar $=1 \mu \mathrm{m})$. (G and $\mathrm{H}$ ) Quantification of mitochondrial abnormalities revealed a significant increase in 3-m blast mice at 7 DPI (G) and 30 DPI (H) as compared to sham control in cortex, hippocampus, and striatum. One tailed unpaired t test, sham vs 3-m 7 DPI cortex, $\mathrm{P}=0.0003$; sham vs 3-m 7 DPI hippocampus; $\mathrm{P}<0.05 ; \mathrm{n}=5$ for sham; $\mathrm{n}=4$ for 3 -m blast at $7 \mathrm{DPI} ; \mathrm{n}=4$ for 3 - $\mathrm{m}$ blast at 30 DPI.

\subsubsection{Behavioral sequelae of blast injury}

\subsubsection{Neurological deficits after LIB exposure assessed by SNAP test}

Using the current settings and $350 \mathrm{~g}$ of high-energy explosive $\mathrm{C} 4$, blast exposure did not cause significant weight loss over the time course of the observations as compared to sham controls. Significant differences in SNAP assessments occurred as compared to 3-, 4- and 7-m blast groups and sham controls at 1,3, and 7 DPI (Figure 3.11A), indicating that neurological deficits affecting coordination, motor strength, proprioception, gait and mental status occurred after LIB exposure. 


\subsubsection{Compromised novelty-induced motor activity and elevated anxiety levels after}

\section{LIB exposure indicated by open-field test and light-dark box}

The open-field behavioral test was used to assess locomotor activity and anxiety behavior. Locomotor activity, as measured by the total distance traveled during the test, exhibited significant differences between 3-m blast and sham control groups at 3 DPI (Figure 3.11B). Similarly, the 3-m blast group also showed a significant shorter distance traveled in the center of the open-field box, a measure of anxiety level, as compared to sham control at 6 DPI (Figure 3.11C). Additionally, when comparing the distances traveled by individual segments (minute by minute), the data showed that the mice in 3-, 4- and 7-m blast groups travelled significantly shorter distances in the first $2 \mathrm{~min}$ as compared to sham control at 3 DPI (Figure 3.11D), indicating onset of decreased locomotor activities after blast exposure.

The light-dark box assessed both novelty-seeking and anxiety-like behaviors in mice. The 3-m blast group showed a significantly lower number of transitions / crossings between compartments as compared to sham control (Figure 3.11E), indicating high anxiety in the 3-m blast group with a motor inhibition caused by the blast exposure at 5 DPI. Additionally, there was a significant decrease in the total distance traveled by mice exposed to blast at a 3-m distance as compared to sham animals, also suggesting reduced motor activity at this closer blast setting (Figure 3.11F).

\subsubsection{Impaired activities of daily living after LIB exposure evidenced by animal nesting}


Nest building, known to be sensitive to evaluate brain lesions, was used to monitor potential progression of neurobehavioral dysfunction [36]. At 3 to $4 \mathrm{DPI}$, the 3-m blast group had significantly lower nesting scores as compared to 4- and 7-m blast groups, and sham controls, while mice in all other three groups showed nest construction at similar levels (Figure 3.11G). The data indicated that the nesting behavior of mice after blast exposure at these intensity levels was compromised.

\subsubsection{Spatial recognition memory after LIB exposure tested by Barnes maze task with reversal training}

From 22 to 29 DPI, Barnes maze to study spatial learning and memory revealed no significant difference between the 3-m blast and sham control groups in primary latency both in acquisition and reversal learning trials. However, significant increases in primary errors (nose-pokes into non-escape holes) were observed both at acquisition day 3 on 25 DPI and reversal training day 2 on 28 DPI (Figure 3.11H-I). More importantly, in reversal learning phase from 27 to $29 \mathrm{DPI}$, the 3-m blast group showed a higher trend of latency as compared to the sham control. The data indicated that the current 3-m blast conditions induced particular spatial learning and memory deficits. 

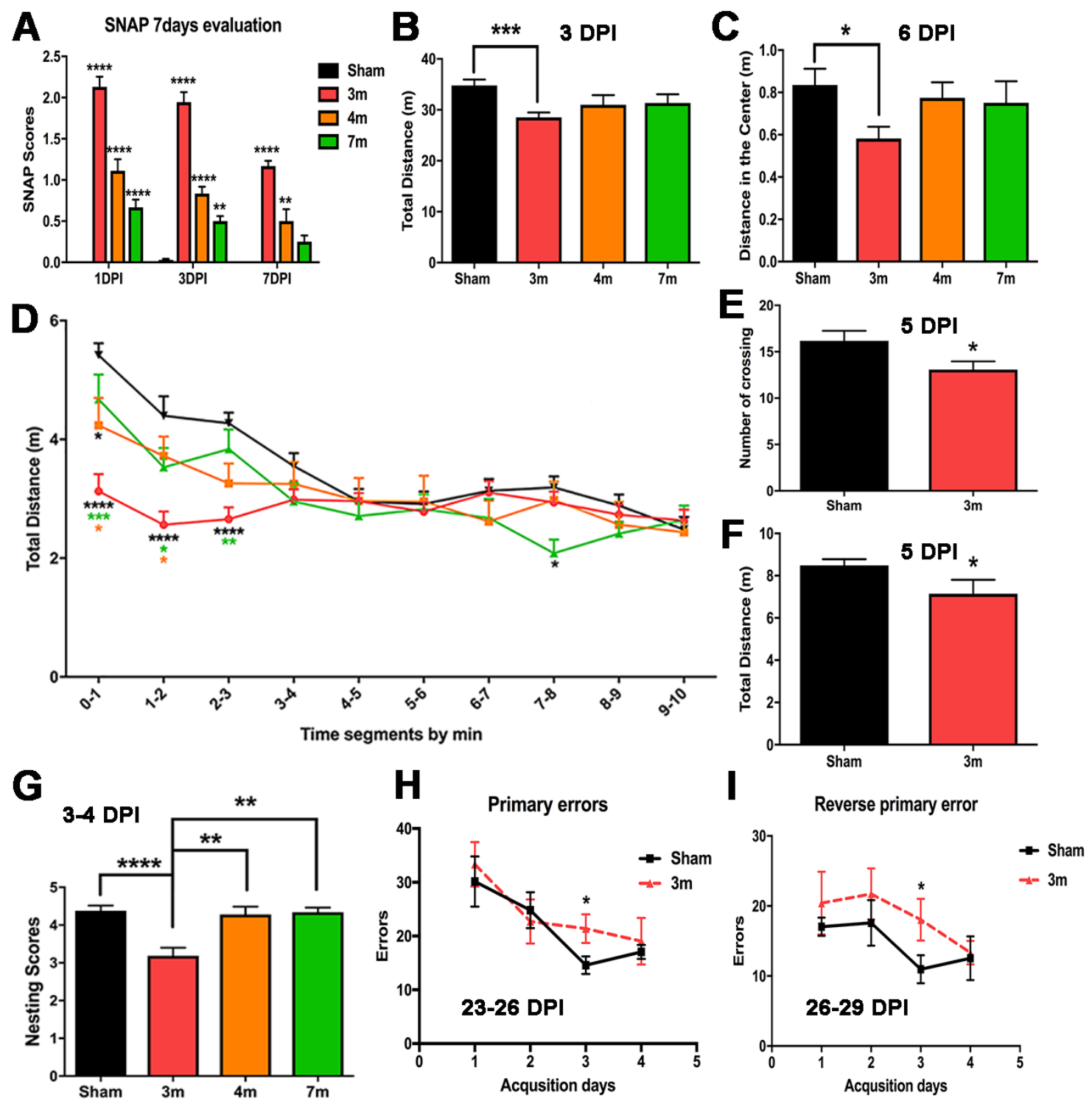

Figure 3.11 Behavioral sequelae of open-field LIB injury.

(A) Neurological status evaluated by SNAP tests on 1 DPI, 3 DPI, and 7 DPI. Two-way ANOVA, $\mathrm{P}<0.0001$ for interaction, all comparisons were performed between blast and sham group on the same DPI; $n=24$ for sham, $n=27$ for $3-m, n=9$ for $4-m$, and $n=12$ for 7-m blast. (B) Open-field test on 3 DPI. Significant difference was identified between 3-m blast and sham groups on total distance traveled. One-way ANOVA: $\mathrm{P}=0.0016$; $\mathrm{n}=24$ for sham, $\mathrm{n}=27$ for $3-\mathrm{m}, \mathrm{n}=9$ for $4-\mathrm{m}$, and $\mathrm{n}=12$ for 7 - $\mathrm{m}$ blast groups. (C) Open- 
field test on 6 DPI. Significant difference was identified between 3-m blast and sham group on distance traveled in the center. One-way ANOVA: $\mathrm{P}=0.0492$. (D) Significant differences were shown among 3-, 4- and 7-m blast, and sham groups when comparing the total distance traveled by time segments $(1 \mathrm{~min})$. Two-way ANOVA: $\mathrm{P}<0.0001$ for interaction, black * indicated comparison between any blast group and sham; orange * indicated comparison between any blast group and 4-m blast group; green * indicated comparison between any blast group and 7-m blast group. The corresponding * was placed under each group. (E-F) Light-dark box test on 5 DPI. Significant differences were identified between 3-m blast and sham group on number of crossing $(E)$ and total distance traveled $(\mathrm{F})$. One tailed unpaired $\mathrm{t}$ test, $\mathrm{P}=0.0181$ for $\mathrm{E}$ and $\mathrm{P}=0.0314$ for $\mathrm{F}$; $\mathrm{n}=12$ for sham, and $\mathrm{n}=15$ for $3-\mathrm{m}$ blast. (G) Animal nesting test. Significant lower nesting scores were identified between 3-m blast and sham, 4- and 7-m blast groups at 34 DPI (One-way ANOVA: $\mathrm{P}<0.0001 ; \mathrm{n}=24$ for sham, $\mathrm{n}=27$ for $3-\mathrm{m}, \mathrm{n}=9$ for $4-\mathrm{m}$, and $\mathrm{n}=12$ for 7-m blast groups), whereas no difference was shown among other group's comparisons. (H-I) Barnes maze with reversal training assessment. Significant increases of primary errors were identified both at acquisition day $3(\mathrm{H})$ and reversal training day 2 (I). One tailed paired $\mathrm{t}$ test, $\mathrm{P}=0.0245$ for $\mathrm{H}$ and $\mathrm{P}=0.0327$ for $\mathrm{I}$.

\subsection{DISCUSSION}

With the large numbers of returning service members diagnosed with TBI, among whom $82.4 \%$ have been classified as mTBI, an urgent need exists to better understand the pathobiology underlying blast-induced neurological deficits and their linkages to blast physics [4]. Animal models are required, in the absence of human observations, to 
address these fundamental questions concerning the pathogenesis of primary blast injury simulating certain later effects of mTBI including anxiety and neurobehavioral dysfunction. While valuable data have been generated based on moderate to severe blast injury animal models, further investigations related to this more common mild concussive injury are needed. Our model employs an open-field detonation of a calibrated C4 explosive to generate low-intensity primary blast waves exhibiting a Friedlander waveform combined with an enhanced dynamic impulse due to the 'ground bounce'. The reflected shock wave interacts with the initial positive phase to produce a higher explosion impact in addition to the blast front in ambient atmosphere. High-speed videography confirms that open-field blast waves freely pass the animals with no apparent head acceleration / rotation or bodily movement. This level of blast energy exposure produced a LIB primary injury in the absence of detectable acceleration effects. Thus the resulting behavioral, pathological and ultrastructural consequences of the blast injury are, in all likelihood, attributable to the primary blast wave effects. Ideally this type of open-field modeling is capable of generating wide and precisely calibrated ranges of primary blast exposure(s). The open-field blast model allows scalable design to accommodate large animal exposures $[8,16,37]$.

The fundamental physics of blast detonation is the rapid chemical decomposition of an explosive into a shock front [8]. The resulting primary blast wave in air travels with supersonic speeds as one of the characteristics of blast waves. The biomechanical injury mechanisms underlying low-intensity primary blast injury have been a challenge for researchers to define. Direct cranial transmission is one of the key mechanisms. When entering the body, the primary blast wave changes through interactions with the 
heterogeneous tissue elements causing dispersion, divergence and attenuation. This is due to the differences in acoustic impedance of tissue types / mediums. Acoustic impedance primarily determines the reflection and transmission of acoustic energy across boundaries of dissimilar density materials. As the shock wave impacting a denser material compresses it, and when it emerges from denser to lighter material it creates tensile deformations $[10,38,39]$. By transducing from air to the brain tissue, the blast pressure magnifies about 1.26 times as the original blast wave overpressure. However, reflection and focusing effects of blast wave appear to be insufficient to change the linear scale to smaller dimensions. If the concentration effects increase the shock intensity by 10 times, this only lowers the expected characteristic length scale of the damage to $0.1-1 \mathrm{~mm}$ as inferred in the original phonon water model; rather a damage range of 30 200 nm was predicted with peaks of $\sim 4 \mathrm{~nm}$, roughly the size of cell membranes [17]. Based on this hypothesis, coupled with brain water composition and a closed intracranial space, a primary blast wave appears to generate specific vibrations at nanometer intervals capable of membrane disruption.

We observed no macroscopic damage of the brain using H\&E staining and no astrogliosis with no significant difference in GFAP expression as an indicator of subsequent astrogliosis between the sham and the 3-m blast groups. Previous studies using shock tubes with higher blast overpressures have shown sustained increase in GFAP expression after blast [40]. By contrast, a recent study using $74.5 \mathrm{kPa}$ blast exposure also showed lack of chronic neuroinflammation in a rat model of repetitive blast injury [41]. A possible explanation for the discrepancy among these findings and our results may relate to the relatively LIB exposure as well as the prone facing animal body 
position frontal to the explosive source. Our most-intense LIB overpressure (46.6 kPa at 3-m distance) is much lower than the blast intensity other studies reported (over $100 \mathrm{kPa}$ ) $[40,42,43]$. Our present experimental design focused on generating LIB exposure scenarios as soldiers are often exposed to such lower levels during deployment or combat exercise. The effects of LIB exposures and primary blast injury have been controversial. A possible reason for not seeing sustained astrogliosis is that our animals were exposed a prone position facing blast with their head and body longitudinally oriented along the direction of shock wave propagation. Soldiers in combat may be prone but also more commonly exposed an upright position facing with their torso toward the front of an incoming shock wave [44]. Prior studies have shown the blast exposure causes more severe damage when the body is in upright as compared to prone position [44, 45]. In these instances, kinetic energy transfer of the primary blast wave may progress through multiple organs and organ systems, including fluid medium (blood) in large blood vessels in the abdomen and chest, finally transmitting pressure into the vessels of the brain [32]. Additionally, shock wave energy transfer into the body can induce systemic injury responses that negatively influence the brain's response to blast. Previous findings under using different animal models and varying exposure scenarios also implicated oxidative stress, vascular changes, inflammation, altered cell adhesion, neuronal, and glial damage/loss in the pathobiology of primary blast injury [32,37]. These findings provided an important basis to our current study. Future directions to assess those temporal profiles of markers may better characterize certain metabolic and systemic responses triggered by primary blast injury. In the current experiment, the ultrastructural damage occurring in 
the prone position provides a novel insight into the brain tissue damage per se in the absence of torso effects.

Injury patterns in the brain after blast exposure are known to be heterogeneous: these include cerebral vasospasm [46], hemorrhage [47], elevated intracranial pressure, and axonal injury with disruption across networks [48], among other effects. In contrast to focal injury, axonal injury occurs in a widespread area and has emerged as one of the most common and important pathological features of TBI [49]. Axons in the white matter, especially at CC, internal capsules, brainstem, $\mathrm{CP}$, and fornix appear to be particularly vulnerable to this type of injury due to the blast energy mechanical loading of the brain during TBI [49]. We have identified different degrees of axonal injury in $\mathrm{CC} \& \mathrm{EC}, \mathrm{CP}$, and fornix detected by silver staining after 3-m, 4-m, and 7-m blast at 7 DPI. These injuries occurred in the absence of macroscopic cell body damage as indicated by the H\&E staining. We also observed concomitant ultrastructural axonal injuries and myelin sheath defects by TEM observation and quantification in CC region in the 3-m group at 7 DPI. We noted progressively increasing intensity of silver staining from the rostral to the caudal cortex of the brain demonstrating that the degree of axonal injury decreases as the pressure wave propagates through the brain from front to back reducing energy. These findings also suggest known links between the clinical frontal lobe injury and behavioral deficits after blast exposures $[12,50]$. In this study, we found no evident axonal injury and myelin sheath defect after LIB exposure at 30 DPI suggesting possible regression of axonal damage. Existing evidence from other related research have also shown possible axonal injury recovery after TBI [51]. In this animal model, due to the blast exposure at very low intensity, healing of damaged axons may 
have occurred by 30 DPI. Potential mechanisms may include replacement of the damaged cytoskeleton, including turnover of microtubules or neurofilaments, and the known process of remyelination [48, 51-54]. These findings require further quantitative studies for confirmation.

The striking mitochondrial abnormalities in the cortex, hippocampus, and striatum of animals exposed to blast at 3-m distance both at 7 DPI and 30 DPI in response to primary LIB exposure in the absence of cell necrosis are unique observations. To the best of our knowledge, this is the first report of unique mitochondrial damage observed by TEM induced by a low-intensity primary blast. Acute mitochondrial damage after blast exposure may lead to or be caused by oxidative stress, decreased cellular energy production, later apoptosis, and impaired neurological function [55]. Blast-induced ultrastructural damages of subcellular organelles have been reported during the acute injury phase after blast [56]. In the present study, damaged mitochondria appear as those with loss of mitochondrial internal cristae, including SCM (low electron dense mitochondria matrix), SDM (high electron dense matrix and swollen intact or fragmented cristae), and DDM (high electron density of matrix and membranes) [33]. Mitochondrial damage frequency after 3-m blast exposure was unevenly distributed among different brain regions: hippocampal mitochondria were more vulnerable at 7 DPI as compared to cortex and striatum, however damaged cortical mitochondria persisted at 30 DPI. Persistent mitochondrial dysfunction and elicited oxidative stress appear to be intimately involved in the pathogenesis of neurodegenerative diseases [57]. This type of subcellular injury may be one of the possible mechanisms accounting for an increased risk of dementia after moderate to severe TBI $[58,59]$. Overall, our current findings have 
identified varying degrees of axonal injury and mitochondrial damage at 7 DPI and 30 DPI as a result of low-intensity, non-impact, non-accelerative blast injury. Future experiments, using increased level of blast exposures, repetitive blasts, and long-term observations, will better characterize linkages between primary blast injury and later chronic mTBI effects and neurodegenerative diseases, as well as providing insights into pathogenic mechanisms. Future investigations are in progress to assess mitochondrial biochemical changes caused by primary blast injury and characterize the potential mitochondrial functional impairment and bioenergetic failure.

Varying neurobehavioral outcomes have been reported following blast-induced mTBI both in clinical and basic research. Clinical reports describe post-concussive stress and depression symptoms along with worse cognitive performance in subjects with blastinduced mTBI, both in acutely (0-7 days) and in long-term (6 months to 5 years) followup [60-62]. Additional reports confirm that mTBI is positively associated clinically with depression and increased anxiety levels [63]. Animal studies using blast exposures have shown various degrees of motor, anxiety, memory and learning dysfunctions. These have been identified to occur both in short-term (0-7 days) and long-term (>2 weeks) [8, 47]. Our study demonstrates acute neurological dysfunction, decreased motor activity, increased anxiety, compromised nesting behavior, and mild spatial learning- and memory deficits in animals caused by a defined low level of explosive blast at $\sim 46.6 \mathrm{kPa}$. Our results show persisting behavioral dysfunction with some degree of functional recovery of acute outcomes of blast in mice up to 30 DPI. Similar findings have been reported with blast intensity at $74.5 \mathrm{kPa}[8,64,65]$. Importantly, major neurobehavioral deficits were observed only in the most intense 3-m LIB exposure group, but not in the 4-m and 7-m 
blast groups indicating a graded response of neurobehavioral changes to the intensity of blast exposure. Specifically, the 3-m blast animals sustained $\sim 6.8$ PSI overpressure with an impulse of $\sim 8.7$ PSI $x \mathrm{~ms}$, which is significantly higher than the 4-m exposure with an approximately 4.6 PSI overpressure with a 6.9 PSI x ms impulse and the 7-m exposure (overpressure: 2.8 PSI, impulse: 4.3 PSI x ms) (Figure 3.3). These associated behavioral changes appear to comport with observed ultrastructural findings. The present open-field blast setting is above 5 PSI, an known threshold for the rupture of the tympanic membrane in humans [66] and of the blast capable of inducing neurobehavioral impairments in our study with an impulse $>7$ PSI x ms, a dynamic measure of blast exposure. Additional studies to define full-scale intensity dependence [67] between the blast overpressure / impulse and additional cognitive and behavioral outcomes are needed. As loss of consciousness is an important outcome for evaluating mTBI and posttraumatic stress disorder-related symptoms after blast exposure, studies using ethically approved animal protocols to assess blast effects in conscious, (possibly sedated animals) may be indicated. Such studies may employ enhanced behavioral testing including novel object recognition, prepulse inhibition, contextual and cued fear conditioning [54]. Blast-induced mTBI often remains under-diagnosed due to the lack or subtle symptoms immediately after exposure as well as negative findings on conventional imaging [68]. Thus, clinical diagnosis is commonly delayed and based upon self-reports [60]. Our TEM findings indicate that, nanoscale ultrastructural damage predicted by the phonon hypothesis may be one of the main causes of primary LIB injury $[17,18]$.

Our current observations demonstrate that primary LIB injury comprises an 'invisible' injury by the absence of the macroscopic damage or necrosis in the presence of 
nanoscale ultrastructural injuries accompanied by neurobehavioral dysfunctions. We describe a highly reproducible open-field primary LIB injury animal model which can be scaled up for larger animals without the limitations imposed by shock tube dimensions. Additionally, the resulting neurobehavioral outcomes, pathological identifications, and ultrastructural assessments related to the primary blast wave quantitatively link blast injury magnitude to fundamental biological alterations. Further research is needed to identify specific cellular and neurological changes associated with primary blast, elucidate differences between blast and non-blast TBI, and provide insights into prevention and treatment of blast-induced mTBI. 


\subsection{REFERENCES}

[1] R.G. DePalma, D.G. Burris, H.R. Champion, M.J. Hodgson, Blast injuries, N Engl J Med 352(13) (2005) 1335-42.

[2] R.G. DePalma, Combat TBI: history, epidemiology, and injury modes, Brain Neurotrauma: Molecular, Neuropsychological, and Rehabilitation Aspects, Boca Raton (FL)2015.

[3] S.T. DeKosky, M.D. Ikonomovic, S. Gandy, Traumatic Brain Injury -- Football, Warfare, and Long-Term Effects., New England Journal of Medicine 363(14) (2010) 1293-1296.

[4] R.G. DePalma, S.W. Hoffman, Combat blast related traumatic brain injury (TBI): Decade of recognition; promise of progress, Behav Brain Res (2016).

[5] J.H. Mena, A.I. Sanchez, A.M. Rubiano, A.B. Peitzman, J.L. Sperry, M.I. Gutierrez, J.C. Puyana, Effect of the modified Glasgow Coma Scale score criteria for mild traumatic brain injury on mortality prediction: comparing classic and modified Glasgow Coma Scale score model scores of 13, J Trauma 71(5) (2011) 1185-92; discussion 1193.

[6] I. Cernak, L.J. Noble-Haeusslein, Traumatic brain injury: an overview of pathobiology with emphasis on military populations, J Cereb Blood Flow Metab 30(2) (2010) 255-66.

[7] R.G. DePalma, G.M. Cross, L. Beck, D. Chandler, Epidemiology of mTBI (mild traumatic brain injury) due to blast: history, DOD/VA data bases: challenges and opportunities, Proceedings of the NATO RTO-MP-HFM-207 Symposium on A Survey of Blast Injury across the Full Landscape of Military Science, 2011, pp. 1-8. 
[8] H. Song, J. Cui, A. Simonyi, C.E. Johnson, G.K. Hubler, R.G. DePalma, Z. Gu, Linking blast physics to biological outcomes in mild traumatic brain injury: Narrative review and preliminary report of an open-field blast model, Behav Brain Res 340 (2018) 147-158.

[9] M.A. Hemphill, S. Dauth, C.J. Yu, B.E. Dabiri, K.K. Parker, Traumatic brain injury and the neuronal microenvironment: a potential role for neuropathological mechanotransduction, Neuron 85(6) (2015) 1177-92.

[10] E. Fievisohn, Z. Bailey, A. Guettler, P. VandeVord, Primary Blast Brain Injury Mechanisms: Current Knowledge, Limitations, and Future Directions, J Biomech Eng (2017).

[11] N. Chandra, A. Sundaramurthy, Acute Pathophysiology of Blast Injury-From Biomechanics to Experiments and Computations: Implications on Head and Polytrauma, in: F.H. Kobeissy (Ed.), Brain Neurotrauma: Molecular, Neuropsychological, and Rehabilitation Aspects, Boca Raton (FL), 2015.

[12] V. Rubovitch, M. Ten-Bosch, O. Zohar, C.R. Harrison, C. Tempel-Brami, E. Stein, B.J. Hoffer, C.D. Balaban, S. Schreiber, W.T. Chiu, C.G. Pick, A mouse model of blastinduced mild traumatic brain injury, Exp Neurol 232(2) (2011) 280-9.

[13] R.A. Bauman, G. Ling, L. Tong, A. Januszkiewicz, D. Agoston, N. Delanerolle, Y. Kim, D. Ritzel, R. Bell, J. Ecklund, R. Armonda, F. Bandak, S. Parks, An introductory characterization of a combat-casualty-care relevant swine model of closed head injury resulting from exposure to explosive blast, J Neurotrauma 26(6) (2009) 841-60. 
[14] K. Feng, L. Zhang, X. Jin, C. Chen, S. Kallakuri, T. Saif, J. Cavanaugh, A. King, Biomechanical Responses of the Brain in Swine Subject to Free-Field Blasts, Front Neurol 7 (2016) 179.

[15] W.G. Proud, The Fundamentals of Blast Physics, Blast Injury Science and Engineering, Springer2016, pp. 3-16.

[16] I. Cernak, D.G. Stein, G.A. Elder, S. Ahlers, K. Curley, R.G. DePalma, J. Duda, M. Ikonomovic, G.L. Iverson, F. Kobeissy, V.E. Koliatsos, M.J. Leggieri, Jr., A.M. Pacifico, D.H. Smith, R. Swanson, F.J. Thompson, F.C. Tortella, Preclinical modelling of militarily relevant traumatic brain injuries: Challenges and recommendations for future directions, Brain Inj 31(9) (2017) 1168-1176.

[17] Y. Kucherov, G.K. Hubler, R.G. DePalma, Blast induced mild traumatic brain injury/concussion: A physical analysis, Journal of Applied Physics 112(10) (2012) 104701.

[18] Y. Kucherov, G. Hubler, J. Michopoulos, B. Johnson, Acoustic waves excited by phonon decay govern the fracture of brittle materials, Journal of Applied Physics 111(2) (2012) 023514.

[19] D. Mott, D. Schwer, T. Young, J. Levine, J.-P. Dionne, A. Makris, G. Hubler, Blastinduced pressure fields beneath a military helmet for non-lethal threats, Proceedings of the 20th International Symposium on Military Aspects of Blast and Shock, 2008.

[20] C.R. DeVore, An improved limiter for multidimensional flux-corrected transport, NAVAL RESEARCH LAB WASHINGTON DC, 1998. 
[21] D.A. Schwer, K. Kailasanath, Numerical simulations of the mitigation of unconfined explosions using water-mist, Proceedings of the Combustion Institute 31(2) (2007) 23612369.

[22] O. Hadass, B.N. Tomlinson, M. Gooyit, S. Chen, J.J. Purdy, J.M. Walker, C. Zhang, A.B. Giritharan, W. Purnell, C.R. Robinson, 2nd, D. Shin, V.A. Schroeder, M.A. Suckow, A. Simonyi, G.Y. Sun, S. Mobashery, J. Cui, M. Chang, Z. Gu, Selective inhibition of matrix metalloproteinase-9 attenuates secondary damage resulting from severe traumatic brain injury, PLoS One 8(10) (2013) e 76904.

[23] R.E. Bennett, D.L. Brody, Acute reduction of microglia does not alter axonal injury in a mouse model of repetitive concussive traumatic brain injury, J Neurotrauma 31(19) (2014) 1647-63.

[24] Z. Chen, D. Shin, S. Chen, K. Mikhail, O. Hadass, B.N. Tomlison, D. Korkin, C.R. Shyu, J. Cui, D.C. Anthony, Z. Gu, Histological quantitation of brain injury using whole slide imaging: a pilot validation study in mice, PLoS One 9(3) (2014) e92133.

[25] A.E. Rizzardi, A.T. Johnson, R.I. Vogel, S.E. Pambuccian, J. Henriksen, A.P. Skubitz, G.J. Metzger, S.C. Schmechel, Quantitative comparison of immunohistochemical staining measured by digital image analysis versus pathologist visual scoring, Diagn Pathol 7 (2012) 42.

[26] G. Paxinos, Paxinos and Franklin's the mouse brain in stereotaxic coordinates, Academic Press2013.

[27] E.S. Musiek, M.M. Lim, G. Yang, A.Q. Bauer, L. Qi, Y. Lee, J.H. Roh, X. OrtizGonzalez, J.T. Dearborn, J.P. Culver, E.D. Herzog, J.B. Hogenesch, D.F. Wozniak, K. Dikranian, B.I. Giasson, D.R. Weaver, D.M. Holtzman, G.A. Fitzgerald, Circadian clock 
proteins regulate neuronal redox homeostasis and neurodegeneration, J Clin Invest 123(12) (2013) 5389-400.

[28] Q. Wang, B. Xiao, S. Cui, H. Song, Y. Qian, L. Dong, H. An, Y. Cui, W. Zhang, Y. He, J. Zhang, J. Yang, F. Zhang, G. Hu, X. Gong, Z. Yan, Y. Zheng, X. Wang, Triptolide treatment reduces Alzheimer's disease (AD)-like pathology through inhibition of BACE1 in a transgenic mouse model of AD, Dis Model Mech 7(12) (2014) 1385-95.

[29] M.J. Barsoum, H. Yuan, A.A. Gerencser, G. Liot, Y. Kushnareva, S. Graber, I. Kovacs, W.D. Lee, J. Waggoner, J. Cui, A.D. White, B. Bossy, J.C. Martinou, R.J. Youle, S.A. Lipton, M.H. Ellisman, G.A. Perkins, E. Bossy-Wetzel, Nitric oxide-induced mitochondrial fission is regulated by dynamin-related GTPases in neurons, EMBO J 25(16) (2006) 3900-11.

[30] J.O. Ojo, C. Bachmeier, B.C. Mouzon, R. Tzekov, M. Mullan, H. Davies, M.G. Stewart, F. Crawford, Ultrastructural Changes in the White and Gray Matter of Mice at Chronic Time Points After Repeated Concussive Head Injury, J Neuropathol Exp Neurol 74(10) (2015) 1012-35.

[31] E.S. Johnson, S.K. Ludwin, The demonstration of recurrent demyelination and remyelination of axons in the central nervous system, Acta Neuropathol 53(2) (1981) 938.

[32] J. Gao, H. Yao, X.D. Pan, A.M. Xie, L. Zhang, J.H. Song, A.J. Ma, Z.C. Liu, Alteration of mitochondrial function and ultrastructure in the hippocampus of pilocarpine-treated rat, Epilepsy Res 108(2) (2014) 162-70.

[33] O.J. Castejon, H.V. de Castejon, Structural patterns of injured mitochondria in human oedematous cerebral cortex, Brain Inj 18(11) (2004) 1107-26. 
[34] S.B. Shelton, D.B. Pettigrew, A.D. Hermann, W. Zhou, P.M. Sullivan, K.A.

Crutcher, K.I. Strauss, A simple, efficient tool for assessment of mice after unilateral cortex injury, J Neurosci Methods 168(2) (2008) 431-42.

[35] J.M. Walker, D. Klakotskaia, D. Ajit, G.A. Weisman, W.G. Wood, G.Y. Sun, P. Serfozo, A. Simonyi, T.R. Schachtman, Beneficial effects of dietary EGCG and voluntary exercise on behavior in an Alzheimer's disease mouse model, J Alzheimers Dis 44(2) (2015) 561-72.

[36] R.M. Deacon, Assessing nest building in mice, Nat Protoc 1(3) (2006) 1117-9.

[37] G.A. Elder, J.R. Stone, S.T. Ahlers, Effects of low-level blast exposure on the nervous system: is there really a controversy, Front Neurol 5(269.10) (2014) 3389. [38] C.J. Clemedson, A. Jonsson, Transmission of elastic disturbances caused by air shock waves in a living body, J Appl Physiol. 16 (1961) 426-30.

[39] A. Nakagawa, G.T. Manley, A.D. Gean, K. Ohtani, R. Armonda, A. Tsukamoto, H. Yamamoto, K. Takayama, T. Tominaga, Mechanisms of primary blast-induced traumatic brain injury: insights from shock-wave research, J Neurotrauma 28(6) (2011) 1101-19. [40] B.R. Huber, J.S. Meabon, T.J. Martin, P.D. Mourad, R. Bennett, B.C. Kraemer, I. Cernak, E.C. Petrie, M.J. Emery, E.R. Swenson, C. Mayer, E. Mehic, E.R. Peskind, D.G. Cook, Blast exposure causes early and persistent aberrant phospho- and cleaved-tau expression in a murine model of mild blast-induced traumatic brain injury, J Alzheimers Dis 37(2) (2013) 309-23.

[41] M.A. Gama Sosa, R. De Gasperi, G.S. Perez Garcia, H. Sosa, C. Searcy, D. Vargas, P.L. Janssen, G.M. Perez, A.E. Tschiffely, W.G. Janssen, R.M. McCarron, P.R. Hof, F.G. Haghighi, S.T. Ahlers, G.A. Elder, Lack of chronic neuroinflammation in the absence of 
focal hemorrhage in a rat model of low-energy blast-induced TBI, Acta Neuropathol Commun 5(1) (2017) 80.

[42] F. Ahmed, S. Plantman, I. Cernak, D.V. Agoston, The Temporal Pattern of Changes in Serum Biomarker Levels Reveals Complex and Dynamically Changing Pathologies after Exposure to a Single Low-Intensity Blast in Mice, Front Neurol 6 (2015) 114. [43] M. Beamer, S.R. Tummala, D. Gullotti, C. Kopil, S. Gorka, A. Ted, C.R. Bass, B. Morrison, 3rd, A.S. Cohen, D.F. Meaney, Primary blast injury causes cognitive impairments and hippocampal circuit alterations, Exp Neurol 283(Pt A) (2016) 16-28. [44] I. Cernak, Blast Injuries and Blast-Induced Neurotrauma: Overview of Pathophysiology and Experimental Knowledge Models and Findings, in: F.H. Kobeissy (Ed.), Brain Neurotrauma: Molecular, Neuropsychological, and Rehabilitation Aspects, Boca Raton (FL), 2015.

[45] I.G. Bowen, E.R. Fletcher, D. Richmond, Estimate of Man's Tolerance to the Direct Effects of Air Blas., U.S. Defense Atomic Support Agency, Washington, D.C., 1968, p. 37.

[46] R.A. Armonda, R.S. Bell, A.H. Vo, G. Ling, T.J. DeGraba, B. Crandall, J. Ecklund, W.W. Campbell, Wartime traumatic cerebral vasospasm: recent review of combat casualties, Neurosurgery 59(6) (2006) 1215-1225.

[47] I. Cernak, A.C. Merkle, V.E. Koliatsos, J.M. Bilik, Q.T. Luong, T.M. Mahota, L. $\mathrm{Xu}$, N. Slack, D. Windle, F.A. Ahmed, The pathobiology of blast injuries and blastinduced neurotrauma as identified using a new experimental model of injury in mice, Neurobiol Dis 41(2) (2011) 538-51. 
[48] V.E. Johnson, W. Stewart, D.H. Smith, Axonal pathology in traumatic brain injury, Exp Neurol 246 (2013) 35-43.

[49] C.L. Mac Donald, A.M. Johnson, D. Cooper, E.C. Nelson, N.J. Werner, J.S.

Shimony, A.Z. Snyder, M.E. Raichle, J.R. Witherow, R. Fang, Detection of blast-related traumatic brain injury in US military personnel, New England Journal of Medicine 364(22) (2011) 2091-2100.

[50] M.L. Lipton, E. Gellella, C. Lo, T. Gold, B.A. Ardekani, K. Shifteh, J.A. Bello, C.A. Branch, Multifocal white matter ultrastructural abnormalities in mild traumatic brain injury with cognitive disability: a voxel-wise analysis of diffusion tensor imaging, $\mathrm{J}$ Neurotrauma 25(11) (2008) 1335-42.

[51] R.C. Armstrong, A.J. Mierzwa, C.M. Marion, G.M. Sullivan, White matter involvement after TBI: Clues to axon and myelin repair capacity, Exp Neurol 275 Pt 3 (2016) 328-333.

[52] X.H. Chen, D.F. Meaney, B.N. Xu, M. Nonaka, T.K. McIntosh, J.A. Wolf, K.E. Saatman, D.H. Smith, Evolution of neurofilament subtype accumulation in axons following diffuse brain injury in the pig, J Neuropathol Exp Neurol 58(6) (1999) 588-96. [53] M.D. Tang-Schomer, V.E. Johnson, P.W. Baas, W. Stewart, D.H. Smith, Partial interruption of axonal transport due to microtubule breakage accounts for the formation of periodic varicosities after traumatic axonal injury, Exp Neurol 233(1) (2012) 364-72. [54] A. Przekwas, M.R. Somayaji, R.K. Gupta, Synaptic Mechanisms of Blast-Induced Brain Injury, Frontiers in Neurology 7 (2016).

[55] J.B. Hiebert, Q. Shen, A.R. Thimmesch, J.D. Pierce, Traumatic brain injury and mitochondrial dysfunction, Am J Med Sci 350(2) (2015) 132-8. 
[56] I. Cernak, Z. Wang, J. Jiang, X. Bian, J. Savic, Ultrastructural and functional characteristics of blast injury-induced neurotrauma, J Trauma 50(4) (2001) 695-706. [57] M.T. Lin, M.F. Beal, Mitochondrial dysfunction and oxidative stress in neurodegenerative diseases, Nature 443(7113) (2006) 787-95.

[58] S. Shively, A.I. Scher, D.P. Perl, R. Diaz-Arrastia, Dementia resulting from traumatic brain injury: what is the pathology?, Arch Neurol 69(10) (2012) 1245-51. [59] J.P. Hayes, M.W. Logue, N. Sadeh, J.M. Spielberg, M. Verfaellie, S.M. Hayes, A. Reagan, D.H. Salat, E.J. Wolf, R.E. McGlinchey, W.P. Milberg, A. Stone, S.A. Schichman, M.W. Miller, Mild traumatic brain injury is associated with reduced cortical thickness in those at risk for Alzheimer's disease, Brain 140(3) (2017) 813-825.

[60] H. Terrio, L.A. Brenner, B.J. Ivins, J.M. Cho, K. Helmick, K. Schwab, K. Scally, R. Bretthauer, D. Warden, Traumatic brain injury screening: preliminary findings in a US Army Brigade Combat Team, J Head Trauma Rehabil 24(1) (2009) 14-23.

[61] C.L. Mac Donald, O.R. Adam, A.M. Johnson, E.C. Nelson, N.J. Werner, D.J. Rivet, D.L. Brody, Acute post-traumatic stress symptoms and age predict outcome in military blast concussion, Brain 138(Pt 5) (2015) 1314-26.

[62] C.L. Mac Donald, J. Barber, M. Jordan, A.M. Johnson, S. Dikmen, J.R. Fann, N. Temkin, Early Clinical Predictors of 5-Year Outcome After Concussive Blast Traumatic Brain Injury, JAMA Neurol 74(7) (2017) 821-829.

[63] R.D. Vanderploeg, H.G. Belanger, R.D. Horner, A.M. Spehar, G. Powell-Cope, S.L. Luther, S.G. Scott, Health outcomes associated with military deployment: mild traumatic brain injury, blast, trauma, and combat associations in the Florida National Guard, Arch Phys Med Rehabil 93(11) (2012) 1887-95. 
[64] G.A. Elder, N.P. Dorr, R. De Gasperi, M.A. Gama Sosa, M.C. Shaughness, E. Maudlin-Jeronimo, A.A. Hall, R.M. McCarron, S.T. Ahlers, Blast exposure induces posttraumatic stress disorder-related traits in a rat model of mild traumatic brain injury, $\mathrm{J}$ Neurotrauma 29(16) (2012) 2564-75.

[65] G. Perez-Garcia, R. De Gasperi, M.A. Gama Sosa, G.M. Perez, A. Otero-Pagan, A. Tschiffely, R.M. McCarron, S.T. Ahlers, G.A. Elder, S. Gandy, PTSD-Related Behavioral Traits in a Rat Model of Blast-Induced mTBI Are Reversed by the mGluR2/3 Receptor Antagonist BCI-838, eneuro 5(1) (2018).

[66] C. Stewart, A. Jagoda, J.M. Howell, Blast injuries: preparing for the inevitable, Emergency Medicine Practice+ Em Practice Guidelines Update 8(4) (2006) 1-26.

[67] I. Cernak, Animal models of head trauma, NeuroRx 2(3) (2005) 410-22.

[68] H.S. Levin, E. Wilde, M. Troyanskaya, N.J. Petersen, R. Scheibel, M. Newsome, M. Radaideh, T. Wu, R. Yallampalli, Z. Chu, X. Li, Diffusion tensor imaging of mild to moderate blast-related traumatic brain injury and its sequelae, J Neurotrauma 27(4) (2010) 683-94. 


\section{CHAPTER 4 MULTIFOCAL NEURONAL}

\section{ULTRASTRUCTURAL ABNORMALTIES AND SYNAPTIC \\ ALTERATIONS IN MICE AFTER LOW-INTENSITY}

\section{BLAST EXPOSURE}

\subsection{ABSTRACT}

Service members during military actions or combat training frequently are exposed to primary blast generated by explosive weaponry. The majority of military-related neurotrauma are classified as mild and designated as "invisible injuries" that is prevalent during current conflicts. While the previous experimental blast injury studies using moderate- to high-intensity exposures focused mainly on gross and microscopic neuropathology, our prior studies have shown that low-intensity blast (LIB) exposures resulted in nanoscale subcellular myelin and mitochondrial damages and subsequent behavioral disorders in the absence of gross or detectable cellular damage. In this study, we used transmission electron microscopy to delineate the LIB effects at the ultrastructural level specifically focusing on the neuron perikaryon, axons, and synapses in the cortex and hippocampus of mice at 7 and 30 days post injury (DPI). We found dysmorphic dark neuronal perikaryon and "cytoplasmic aeration" of dendritic processes, as well as increased microtubular fragmentation of the myelinated axons along with biochemically measured elevated tau/phosphorylated tau/A $\beta$ levels. The number of cortical excitatory synapses decreased along with a compensatory increase of the postsynaptic density (PSD) thickness both at 7 and 30 DPI. However, the amount of 
hippocampal CA1 synapses increased with the reduced PSD thickness. Additionally, we observed a significant increase in protein levels of PSD95 and synaptophysin mainly at 7 DPI indicating potential synaptic reorganization. These results demonstrated that a single LIB exposure can lead to ultrastructural brain injury with accompanying multifocal neuronal organelle alterations. This preclinical study provides key insights into disease pathogenesis related to primary blast exposure.

\subsection{INTRODUCTION}

Traumatic brain injury is of a major concern to military personnel due to frequent enemy use of improvised explosive devices [1], as well as exposures to explosive weaponry during occupational military training. During the Operation Iraqi Freedom/Operation Enduring Freedom (OIF/OEF), the US army forces reported that over $75 \%$ of all combat victims were due to blast exposures. Most of those combat-related traumatic brain injuries (TBI) have been classified as mild injuries. The US Department of Defense (DoD), from the compiled 2000-2018 Q1 data, reported up to $82.3 \%$ cases of all the TBI are mild TBI (mTBI) in combat veterans. Currently, mTBI is defined as loss of consciousness for less than 30 minutes or state of confusion and/or memory loss, which does not exceed 24 hours [2], and is associated with a normal brain computed tomography (CT) scan. However, clinical studies of outcomes after mTBI report signs of neurodegenerative and mental health disorders in many of these casualties [3].

While prior studies have provided insights into TBI mechanisms with moderateto high-intensity blast exposures over $100 \mathrm{kPa}$ peak overpressure, the pathobiology underlying the impact of primary low-intensity blast (LIB)-induced brain injury requires 
further clarification $[4,5]$. We have previously established an open-field LIB mouse model, with exposure to a magnitude of $46.6 \mathrm{kPa}$ peak overpressure and maximum impulse of 8.7 PSI (aka $60 \mathrm{kPa}) \times$ ms blast. In the absence of head motion, animals experienced no mortality, gross bodily injuries, or glial response [4, 6, 7]. Our prior studies and physical model suggested that primary LIB exposure leads to a nanoscale ultrastructural brain injury probably due to transmission of a pressure wave travelling to and through the brain with energy sufficient to induce shearing force subcellular damage $[4,6-8]$.

Using transmission electron microscopy (TEM), we previously demonstrated neuropathological changes of myelin and mitochondria associated with behavioral impairments after a primary LIB. Similarly, myelin and mitochondrial morphological changes have also been induced by weight drop or controlled cortical impact (CCI) models $[9,10]$. White matter damage typified by localized axonal degeneration, microglial phagocytosis, and increased neuroglial cell density have been described after repetitive concussive head injury induced by CCI [11]. Other reports demonstrated, in the cerebral cortex of wild type and hTau mice, evidence of dark neurons, subtle changes to the microvasculature, along with a mild augmentation of age-related features such as lipofuscin deposition and electron-dense inclusions in microglial and perivascular cells [11]. In contrast to focal impact related TBI, blast-induced mTBI appears to cause more diffuse neuropathological alterations [12]. Thus, blast exposure may cause diffuse breakdown of microtubules and neurofilaments affecting not only the axon but other neuronal ultrastructural elements also, spreading from the perikaryon to the synapse [13, 14]. 
The present report broadens our prior ultrastructural characterization of LIB exposure and further explores the distinct pathological hallmark features of primary blast injury $[6,15]$. Specifically, we focused on ultrastructural examinations in cortex and hippocampus at 7 and 30 days post injury (DPI). We tested the hypothesis that LIB exposure results in multifocal ultrastructural damage in the neuron using TEM at various resolutions in conjunction with biochemical analysis of tau/phosphorylated tau (ptau)/A $\beta$ levels. This preclinical study further confirms that a single LIB induces multiple and diffuse ultrastructural changes likely leading to long-term neurobehavioral impairments $[4,6,7]$, providing key insights into disease pathogenesis related to primary blast exposure.

\subsection{METHOD}

\subsubsection{Materials}

The chemicals were purchased from Sigma-Aldrich (St. Louis, MO, USA). General anesthesia was performed using Ketamine hydrochloride injection $(100 \mathrm{mg} / \mathrm{mL}, 1044102$, Henry Schein, Melville, NY, USA), and Xylazine hydrochloride injection (100mg/mL, 13985-704-10, Vet One, Boise, ID, USA). Primary fixative (Electron Microscopy Sciences, Hartfield, PA, USA) for TEM contained 100 mM Sodium Cacodylate, 2\% glutaraldehyde, and $2 \%$ paraformaldehyde. Reagents for BCA protein assay was purchased from Thermo Scientific (Rockford, IL, USA).

\subsubsection{Animals and open-field blast setting}


As described previously $[4,6]$, thirty-three male C57BL/6J mice (The Jackson

Laboratory, Bar Harbor, ME) at 2 months of age were housed four animals per cage, with a 12-h light/dark cycle (lights on at 7:00 AM) and unrestricted access to food and water. A cotton pad and an environmental enrichment toy (small plastic house) were placed in each cage. All animals were observed for good health before, during, and after testing. All experimental groups in the studies were handled in a randomized, double-blinded manner. All procedures were carried out in accordance to the University of Missouri approved protocols for the Care and Use of Laboratory Animals and the ARRIVE guidelines.

Based on the blast exposure setting, animals were randomly assigned into the 3-m blast and sham control groups. For TEM studies, animals with 3-m blast were further randomly divided into two sub-groups as 7 DPI $(n=4)$ and 30 DPI $(n=4)$, and tested with their corresponding sham controls $(\mathrm{n}=5)$. For biochemical studies, animals with a 3-m blast exposure were randomly grouped into four sub-groups as $3 \mathrm{hrs}(\mathrm{n}=4), 24 \mathrm{hrs}(\mathrm{n}=4)$, 7 DPI $(n=4)$, and 30 DPI $(n=4)$, with corresponding sham controls $(n=4)$.

Mice were anesthetized with intraperitoneal (i.p.) injection of $5 \mu \mathrm{l} / \mathrm{g}$ body weight of Ketamine/Xylazine mixture ( $25 \mathrm{mg} / \mathrm{mL}$ Ketamine and $1.25 \mathrm{mg} / \mathrm{mL}$ Xylazine). Sham group underwent the identical anesthesia procedures only without blast exposure. The animals were placed in the prone position facing the blast generated by detonating $350 \mathrm{~g}$ of $\mathrm{C} 4$ at the 3-m distance platforms. The animals were not restrained within the holding apparatus. Following blast wave exposure, animals were returned to their original cages. After recovery from anesthesia, mice were continuously monitored for at least 15-30 min, 
and spontaneous movement was observed in all instances. Animals were allowed access to food and water ad libitum before and after blast exposure.

\subsubsection{Transmission Electron Microscopy study}

\subsubsection{Tissue preparation}

Animals used for TEM study were sacrificed at 7 and 30 DPI. The samples were collected and processed as previously described [6]. Briefly, anesthetized mice were perfused trans-cardially with saline $(0.9 \% \mathrm{NaCl})$ containing heparin $(20$ units $/ \mathrm{ml})$ for 2 minutes at $35^{\circ} \mathrm{C}$ and then with $4 \%$ paraformaldehyde at $4^{\circ} \mathrm{C}$ for 2 min. Brains were dissected and tissue sections were cut at 1-mm thickness (approximately 6 coronal tissue blocks per brain) with the brain matrix (Ted Pella, Inc., Altadena, CA, USA), and postfixed in primary fixative (Electron Microscopy Sciences, Hartfield, PA, USA) which contained $100 \mathrm{mM}$ sodium cacodylate, $2 \%$ glutaraldehyde, and 2\% paraformaldehyde. Brain sections (approximate coordinates from bregma $-1.70 \mathrm{~mm}$ to $-2.70 \mathrm{~mm}$ ) were further stereologically cut into $1 \mathrm{~mm}^{3}$ size from cerebral cortex (including corpus callosum), hippocampus (CA1), and striatum under light microscopy. Tissues were kept in $0.1 \mathrm{M} \mathrm{NaCacodylate} \mathrm{buffer}(\mathrm{pH}=7.4)$ with $0.13 \mathrm{M}$ sucrose all the time. Next, the tissues were embedded using 1\% osmium tetroxide (Ted Pella, Inc. Redding, California) in Cacodylate buffer. After embedding of TEM tissue blocks, each block was trimmed approximately $150 \mu \mathrm{m}$ deep and the surrounding tissue to avoid potential artifacts. An area measuring approximately $250 \mu \mathrm{m}$ x $200 \mu \mathrm{m}$ of tissue block was selected for the further sectioning. Since examination of consecutive thin sections could result in repeated measurement of the same cell and features, non-consecutive sections were collected at a thickness of $85 \mathrm{~nm}$ using an ultramicrotome (Ultracut UCT, Leica Microsystems, 
Germany) and a diamond knife (Diatome, Hatfield PA), and placed on grids for four sections per grid. Ten grids were prepared randomly within approximately $450 \mu \mathrm{m}$ per block. Four grids randomly selected and were iteratively prepared for examination. Images were acquired with a JEOL JEM 1400 transmission electron microscope (JEOL, Peabody, MA) at $80 \mathrm{kV}$ on a Gatan Ultrascan $1000 \mathrm{CCD}$ (Gatan, Inc, Pleasanton, CA). Collection of unique data on these four grids of each region was ensured by proceeding systematically and randomly across each grid and marking each examined region on the stage using the JEOL software.

\subsubsection{Neuronal cell body and neurofilament changes identification and quantification}

Neuronal cell bodies and microtubules / neurofilaments changes within the axoplasm of myelinated axons were examined for abnormalities. Normal neuronal cell bodies (perikaryon) can be distinguished from the neuropil by its well demarcated cytoplasm filled with Nissl bodies, from which dendritic protrusions depart. Normal axonal microtubules are identifiable as long rod-like structures in longitudinal sections and as evenly distributed puncta on transverse sections [16]. By contrast, abnormal microtubules and neurofilaments were characterized by the dense change (aggregation of microtubules), loss or irregular repartition of the microtubules, and presence of vacuoles occupying more than $30 \%$ of the cytoplasm content [17]. To characterize changes in neuronal cell bodies, we systematically examined within 10 different counting frames at $800 \mathrm{x}$ magnification covering an area over $32,000 \mu \mathrm{m}^{2}$ in each brain region per animal to eliminate possible selection bias. For analysis of axonal microtubular and neurofilament 
changes, and average 35 TEM images at 2,000 x magnifications were randomly selected and captured from the cortex and hippocampus, respectively.

\subsubsection{Synaptic changes identification and quantification}

In this study, we also focused on the asymmetric (Type I) synapses, the most common subtype of synapses located mainly on dendritic spines and shafts in the mammalian central nervous system [18]. For each animal, four non-consecutive thin sections were arranged in a copper grid for TEM acquisition. The methodology for quantification was based upon previously published studies assessing synaptic vesicular properties [19, 20]. To avoid overlapping capture, we systematically and randomly acquired the images of 5 to 10 different unbiased counting frames in a $800 \mathrm{x}$ magnification from each brain region. In each frame, we randomly captured two pictures at 2,000 x (intermediate magnification), then two to three pictures were randomly captured at 10,000 $\mathrm{x}$ (high magnification) from each picture at 2,000 x. For each animal, on average, 30 randomly selected synapses were captured at 10,000 x (high magnification) and 35 random images at 2,000 x magnifications from the cortex and hippocampus. At 10,000 x magnification, this study covered an area of $\sim 2,500 \mu \mathrm{m}^{2}$ in each brain region. Such high magnified pictures were useful for detailed characterization of synaptic features whereas intermediate magnification was used for the assessment of the numeric synaptic density: total number of asymmetric synapses per area.

At 10,000 x magnification, we assessed the length of active zone (AZ), defined by the thickened presynaptic membrane with electron dense materials in alignment with the post synaptic membrane. This area is the site involved in the process of priming and 
docking of synaptic vesicles [21]. Next, we counted the number of vesicles per synaptic interface. The included synaptic vesicle shapes were round or ovoid measuring 40 to 60 $\mathrm{nm}$ in diameter and located in the area within $300 \mathrm{~nm}$ of the active zone [22]. The vesicles smaller than $40 \mathrm{~nm}$ were excluded to avoid inclusions of polyribosomes and microtubules. Furthermore, we assessed the shortest distance between the vesicle membrane and the active zone for every vesicle. The vesicles located less than $50 \mathrm{~nm}$ away from the active zone were grouped as the docked vesicles or primed for docking $[19,20]$. Thus, we determined the percentage of docked vesicles as the proportion of synaptic vesicles located within $50 \mathrm{~nm}$ of the active zone. At the post synaptic compartment, we measured the thickness of the post synaptic density (PSD) for each synapse. PSD is identified as a fuzzy electron dense structure located at the post synaptic membrane extending $\sim 35$ to $50 \mathrm{~nm}$ into the cytoplasm [21] and is known to contain varieties of receptors and scaffolding proteins involved in synaptic transmission and plasticity [21]. PSD thickness was measured based on the post synaptic grayscale value. The distance between the local maxima and minima grayscale value represented the PSD thickness, one of the hallmarks of asymmetric synapses. At 2,000 x magnification, a total of $\sim 130$ electron micrographs (covering an area of 14,512 $\mu \mathrm{m}^{2}$ ) were used for assessing the numerical synapse density: defined as the total number of asymmetric synapses per area.

All of the above measurements were performed by two individual investigators in a double-blind fashion. We used the number of fields of view counted to perform the statistical analysis to reflect the detailed numbers of observed features. Using NIH ImageJ software, the straight line and the multi-point tools respectively were used for 
measurement and quantification of distinct synaptic ultrastructural features. To assure measurement reliability, all individual investigators, who involved the measurements, received pre-analysis test training for an assessment of inter-rater consistency. The interrater variability was $+/-0.00047$ on quantification of asymmetric (Type I) synapses, indicating a high consistency for the measurement.

\subsubsection{Western blot and analysis of synaptic protein}

The cortices of LIB exposed mice were used for these analyses. The protein samples were added with $4 \times$ SDS sample buffer with 1\% 2-mercaptoethanol and resolved on $12 \%, 1.5 \mathrm{~mm}$ SDS-PAGE gels as previously described [23]. Then proteins in SDS-PAGE gel were transferred to a nitrocellulose membrane. The membrane was first incubated in phosphate-buffered saline plus $0.1 \%$ Tween-20 (PBST) (pH 7.4) containing $5 \%$ non-fat milk for 1 hour at room temperature and then incubated with primary antibody, including PSD95 (MA1-046 Thermo Fisher Scientific, 1:2000); Synaptophysin (AB9272 Millipore, 1:1000); and Actin (A3854 Sigma-Aldrich, $1: 15000$ ) at $4{ }^{\circ} \mathrm{C}$ overnight. After washing with PBST three times, the membrane was incubated with corresponding secondary antibody (anti-mouse IgG, A0168 Sigma-Aldrich, 1:2000; anti-rabbit IgG, A0545 SigmaAldrich, 1:2000) in PBST for 1 hour at room temperature. Immuno-reactive bands were detected using Amersham ECL Prime Western Blotting Detection Reagent and LAS4000 Fujifilm imaging system.

\subsubsection{Meso Scale Discovery (MSD) ELISA}


We used tau-specific ELISA to quantify levels of tau and phosphorylated-tau (ptau) as previously described [24]. The sandwich ELISA used a capture antibody BT2 that was raised against a purified tau protein, allowing a maximum capture of all forms of tau from mouse brain tissue. The ptau at Thr 181 was captured by a specific antibody AT270. The detecting antibody is the biotinylated HT7 that recognizes residue 159-163 of tau. All three tau antibodies were purchased from Thermo Scientific (Rockford, IL). The combination of these antibodies allowed us to quantify alteration of tau and ptau in mouse brains after the blast. ELISA was performed using Meso Scale Discovery platform (MSD, Gaithersburg, MD). The internal calibrators of ptau and tau are purchased from Meso Scale Discovery.

\subsubsection{Statistical analyses}

Statistical analyses were performed using Prism (GraphPad Software). The data in graph were presented in Mean \pm Standard Error of Mean (SEM). Statistical comparisons were made by One-way ANOVA test followed by Tukey test for multiple comparisons. Differences were considered significant at $\mathrm{P}<0.05$ for all analyses, with * indicating $\mathrm{P}<0.05 ; * * \mathrm{P}<0.01 ; * * * \mathrm{P}<0.001 ; * * * * \mathrm{P}<0.0001$.

\subsection{RESULTS}

\subsubsection{Degenerating neuron cell bodies and dendrites were observed after LIB exposure}

Normal characteristics of normal pyramidal neuron cell bodies with pale cytoplasm and nucleus were observed in sham control group samples. The contours of these cells were 
well-defined with characteristic protrusions of dendritic processes. As expected in sham non-injured brain tissue, the cytoplasm was rich in rough endoplasmic reticulum and rosettes of free ribosomes referred to as Nissl bodies were identified. The observed mitochondria were ovoid with a dense matrix containing organized cristae. These features were shown on Figure 4.1A at 800 x magnification. By contrast, after LIB exposure, profiles of degenerated pyramidal neuron cell bodies were notable. These were readily recognizable by a dark cytoplasm, multiples swollen and clear mitochondria, and distorted endoplasmic reticulum (Figure 4.1B-C). The mitochondria appeared swollen with an electron-lucent matrix containing disarranged and fragmented cristae. We also noticed an enlargement of the perinuclear space (Figure 4.1C) suggesting an impairment in the nucleus-cytoplasm communication. Moreover, the dendritic processes harbored cytoskeletal abnormalities characterized by a disappearance of microtubules and neurofilaments within the cytoplasm giving an aspect of clear cytoplasm referred to as "cytoplasmic aeration" (Figure 4.1D). Such features indicative of degeneration in the blast subjects were not observed in the sham group. 

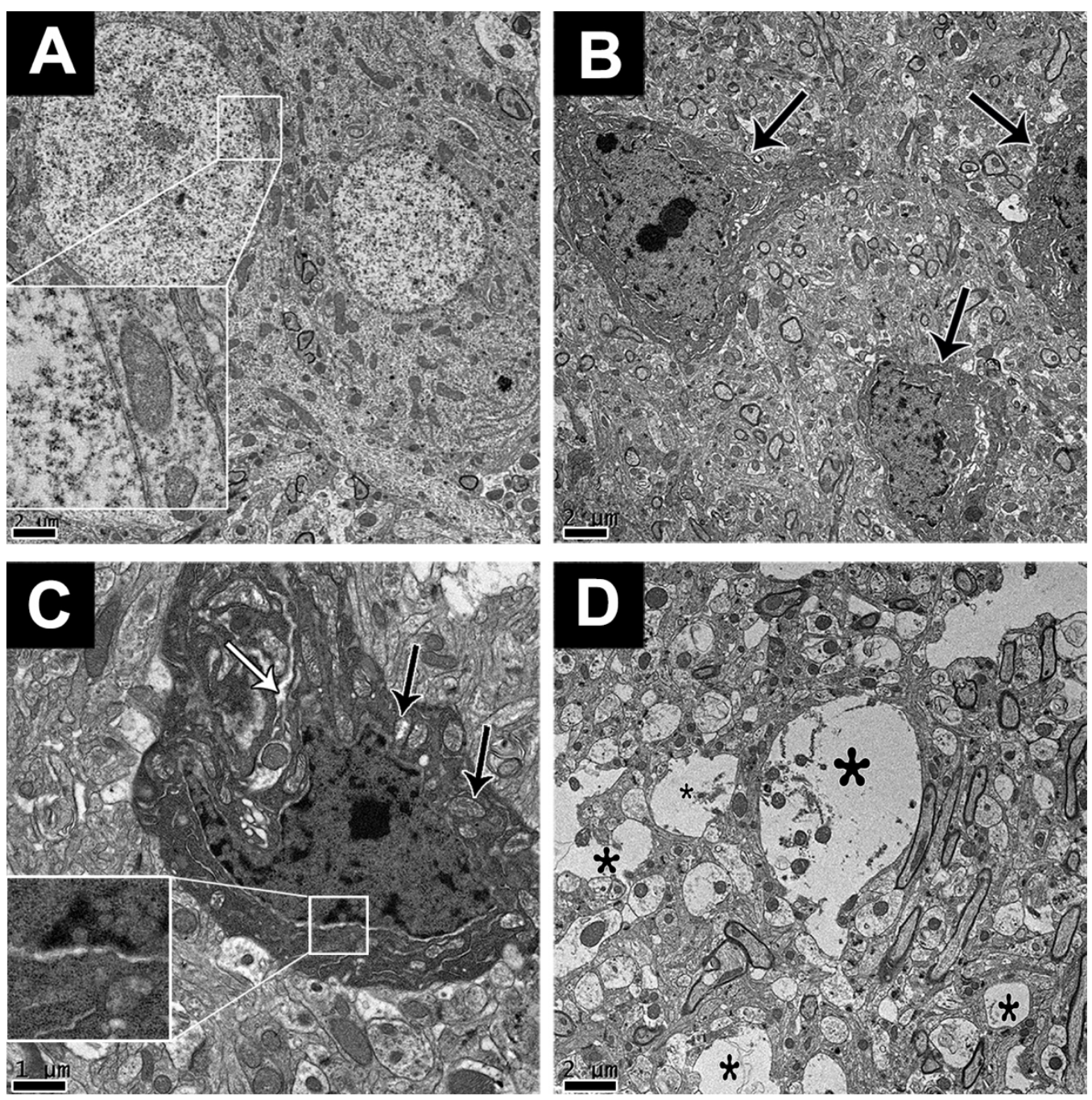

Figure 4.1 Neuronal cell body and dendritic abnormalities after LIB exposure.

(A) Representative TEM images of normal pyramidal neurons in the mouse cortex in the sham control (scale $=2 \mu \mathrm{m}, 800 \mathrm{x}$ magnification). Inset: normal mitochondria with a dense matrix containing organized, deep-protruded cristae, also the normal perinuclear membrane appears regular with close cytoplasmic contact. (B-C) Degenerating neuron cell bodies appeared dark (black arrow in B) with swollen mitochondria (black arrow in C), distended perinuclear space (inset), and distorted endoplasmic reticulum (white arrow) (B: scale $=2 \mu \mathrm{m}, 800 \times$ magnification; $\mathrm{C}$ : scale $=1 \mu \mathrm{m}, 2,000 \times$ magnification, 
respectively). (D) The dendritic processes after blast were swollen with a near total cytoskeletal disappearance, indicated by the asterisk (scale $=2 \mu \mathrm{m}, 800 \mathrm{x}$ magnification).

\subsubsection{Microtubule abnormalities and vacuole formation in the myelinated axons after LIB exposure}

Microtubule ultrastructure integrity is known to be essential for proper axonal transport [25]. The present study examined the effect of LIB exposure on microtubular structure and vacuole presence. Microtubules were identifiable in sham control group as evenly distributed long, rod-like structures on longitudinal section or as puncta on axial transsection within the axoplasm (Figure 4.2A-B). After LIB, we observed multiple features of microtubule degeneration in the myelinated axons.

The appearance of "dark axons," also referred to as microtubule compaction, resulted from the reduction in the distance between microtubules and was also accompanied by the disappearance of most neurofilament side-arms (Figure 4.2C). Additionally, we observed swollen myelinated axons with granular disintegration (Figure 4.2D) and dramatic disorganization of the disrupted axonal microtubules (Figure 4.2EG). Next, we examined the formation of autophagic vacuoles as they play a homeostatic role in the degradation of abnormal axoplasmic proteins. We considered vacuoles abnormal when their sizes exceeded one-third of the axoplasm content (Figure 4.2H). Furthermore, we quantified myelinated axons that displayed features of abnormal microtubules and extended vacuoles within their axoplasm. We found significant increases of vacuole abnormalities at 7 DPI in both cortex and striatum, with a higher trend in the hippocampus (Figure 4.2J-L). 

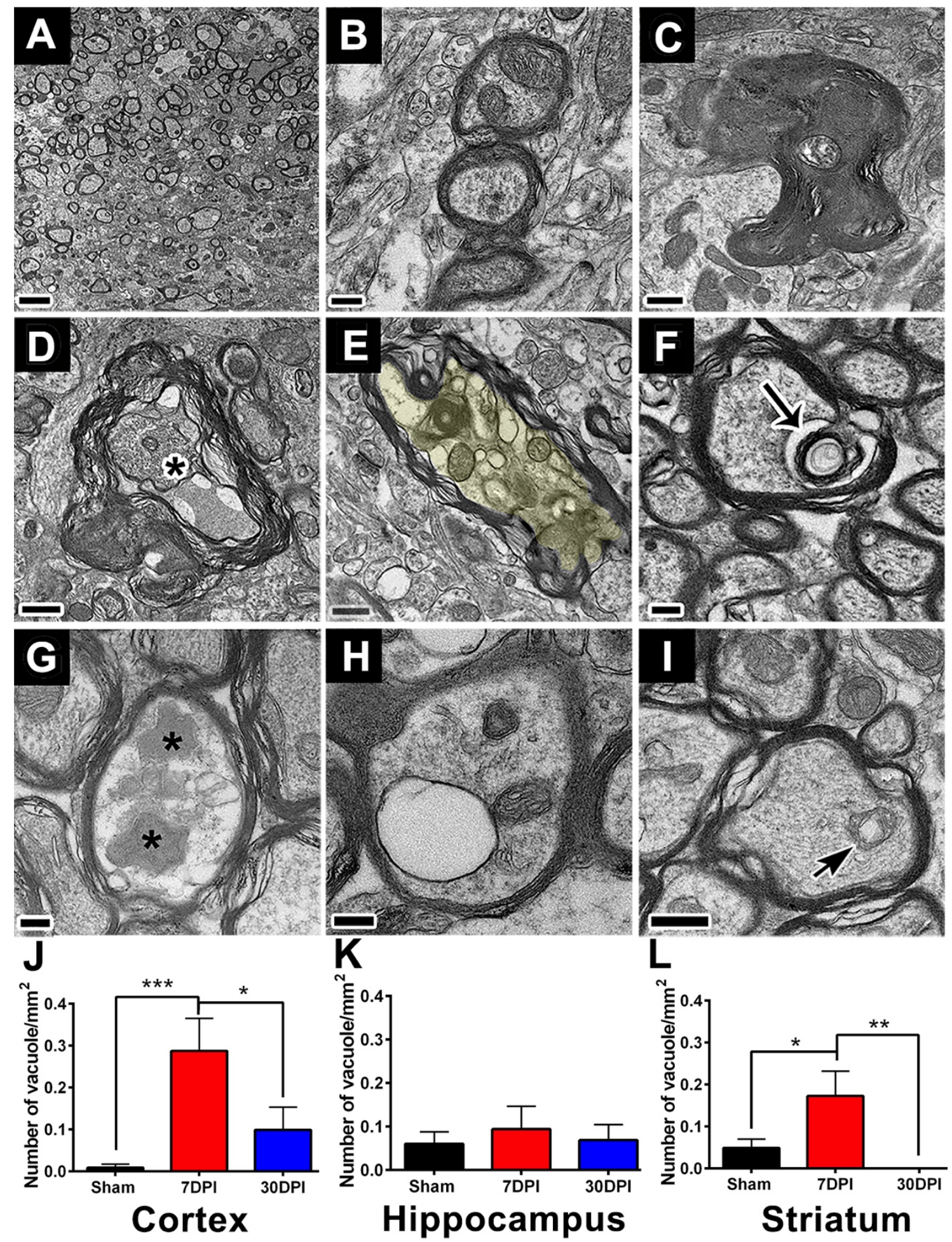

Figure 4.2 Microtubule changes and vacuole formations observed in myelinated axons after LIB exposure. 
(A-B) Representative images of the cortex in sham control group (A: 5,000 x, scale bar $=0.5 \mu \mathrm{m}$; B: 10,000x, scale bar $=0.2 \mu \mathrm{m}$, respectively). (C) Dark axons were observed after LIB (scale bar=0.5 $\mu \mathrm{m}$ ). (D-E) Cytoplasmic degeneration of myelinated axons were identified after LIB at high magnification (scale bar $=0.5 \mu \mathrm{m}$ ). (D) Excessive lamellation of the myelin sheath, heterogeneous changes of the cytoplasm (asterisk) leading to distinct compartments with peripheral vacuolations. (E) Extended lamellation (or splitting) of the myelin sheath, heterogeneous changes of the cytoplasm characterized by vacuoles (yellow), swollen mitochondria, and myelin onion. (F) Intracytoplasmic degeneration of myelin sheath, which was referred as "myelin onion", could be found in the corpus callosum after blast (scale bar=0.2 $\mu \mathrm{m}$ ). (G-I) Cytoskeletal disruptions in myelinated axons, dendritic processes. Aggregations of microtubules (asterisks in G) were seen in myelinated axons after blast (scale bar $=0.2 \mu \mathrm{m}$ ). (H) Large vacuole is a clear well-limited bulb in the cytoplasm related to an autophagic process; these could be identified in any cell type after blast (scale bar $=0.2 \mu \mathrm{m}$ ). (I) Representative image of an early stage of autophagic vacuole formation (scale bar $=0.2 \mu \mathrm{m}$ ). (J-L) Significant increase in microtubule changes and vacuole formations were observed at 7 DPI in the cortex and the striatum. One-way ANOVA followed by Tukey test for multiple comparisons $(\mathrm{P}=0.0001)$.

\subsubsection{LIB induced axonal cytoskeletal injury associated with altered tau phosphorylation and Aß levels}

Since emerging evidence supports the notion that blast injury induces axonal injury associated with tau pathologies and neurodegeneration [26, 27], we measured various 
forms of tau and amyloid beta $(\mathrm{A} \beta)$ in LIB exposed mice. Using tau-specific ELISA to quantify levels of tau and ptau, we have identified significantly elevated levels of total tau after blast at both 7 DPI and 30 DPI in the cortex as compared to sham group (Figure 4.3A). Additionally, ptau, $A \beta 40$, and $A \beta 42$ levels were significantly increased after blast at $30 \mathrm{DPI}$ (Figure 4.3A-B), suggesting alterations of microtubule proteins and transmembrane amyloidic cleavages involved in axonal cytoskeletal deficits after LIB at a $46.6 \mathrm{kPa}$ level.
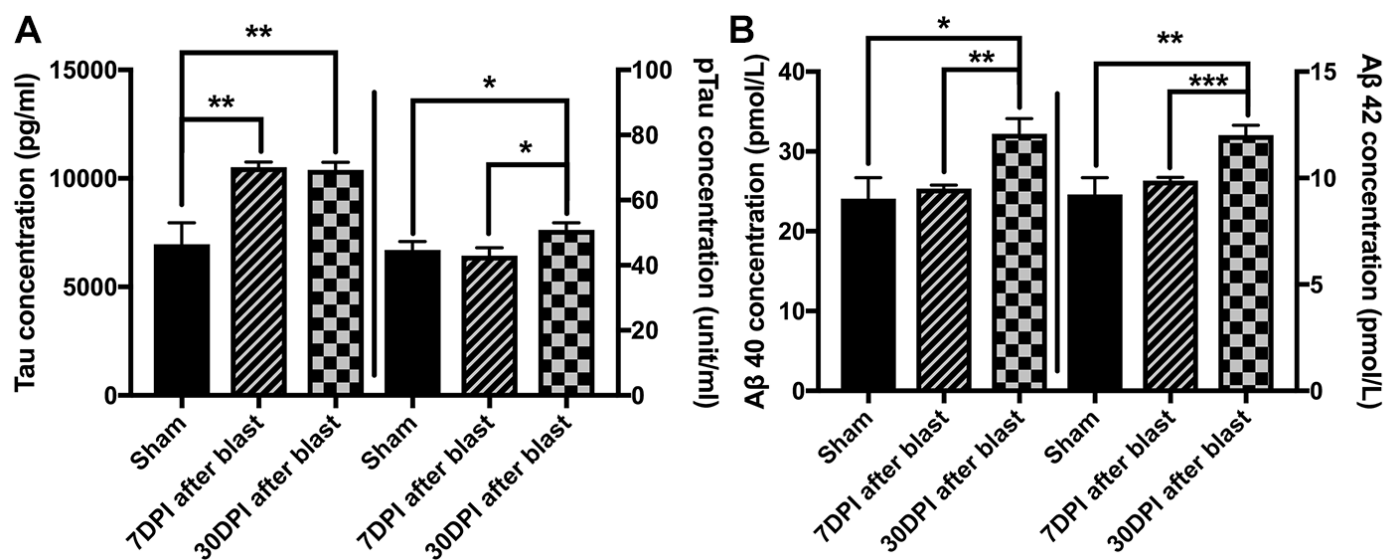

Figure 4.3 LIB induced changes in levels of tau, ptau, and Aß.

(A) Elevated tau levels after blast at 7 DPI $(\mathrm{P}<0.01)$ and 30 DPI $(\mathrm{P}<0.01)$ and increased ptau levels after 3-m blast at 30 DPI $(\mathrm{P}<0.05)$, as compared to sham individually by oneway ANOVA followed by Tukey test for multiple comparisons. (B) Increased levels of $A \beta 40$ and $A \beta 42$ after blast both at 7 and 30 DPI.

\subsubsection{Synaptic changes were observed after LIB exposure}

To link synaptic dysfunctions with neuropathological findings, including neuronal / dendritic changes, microtubule abnormalities, and accumulation of tau and $A \beta$ [28], we investigated LIB effects on the synapse at both ultrastructural and biochemical levels. 
4.4.4.1 The numerical density of asymmetric synapse decreased after LIB exposure in the cortex and increased in the hippocampus

The total number of asymmetric (excitatory) synapse in a brain volume correlates with cognitive and learning abilities [29]. Since our previous study first reported spatial learning and memory deficits associated with LIB exposure [6], here, we examined the effects of LIB exposure on the numerical density of excitatory synapses. In the cortex, the mean numerical density of asymmetric synapses was significantly higher in the sham group $(199,637 \pm 12,438)$ (Figure 4.4A-D) versus blast at 7 DPI $(172,524 \pm 6,904)$ and 30 DPI $(132,937 \pm 6,211)$ groups (Figure 4.4E-G). By contrast, in the hippocampus, we observed significantly less mean numerical density in the sham group $(111,951 \pm 3,965)$ as compared to $7 \mathrm{DPI}(174,873 \pm 7,541)$ and $30 \mathrm{DPI}(185,070 \pm 6,762)$ groups (Figure 4.4H). 

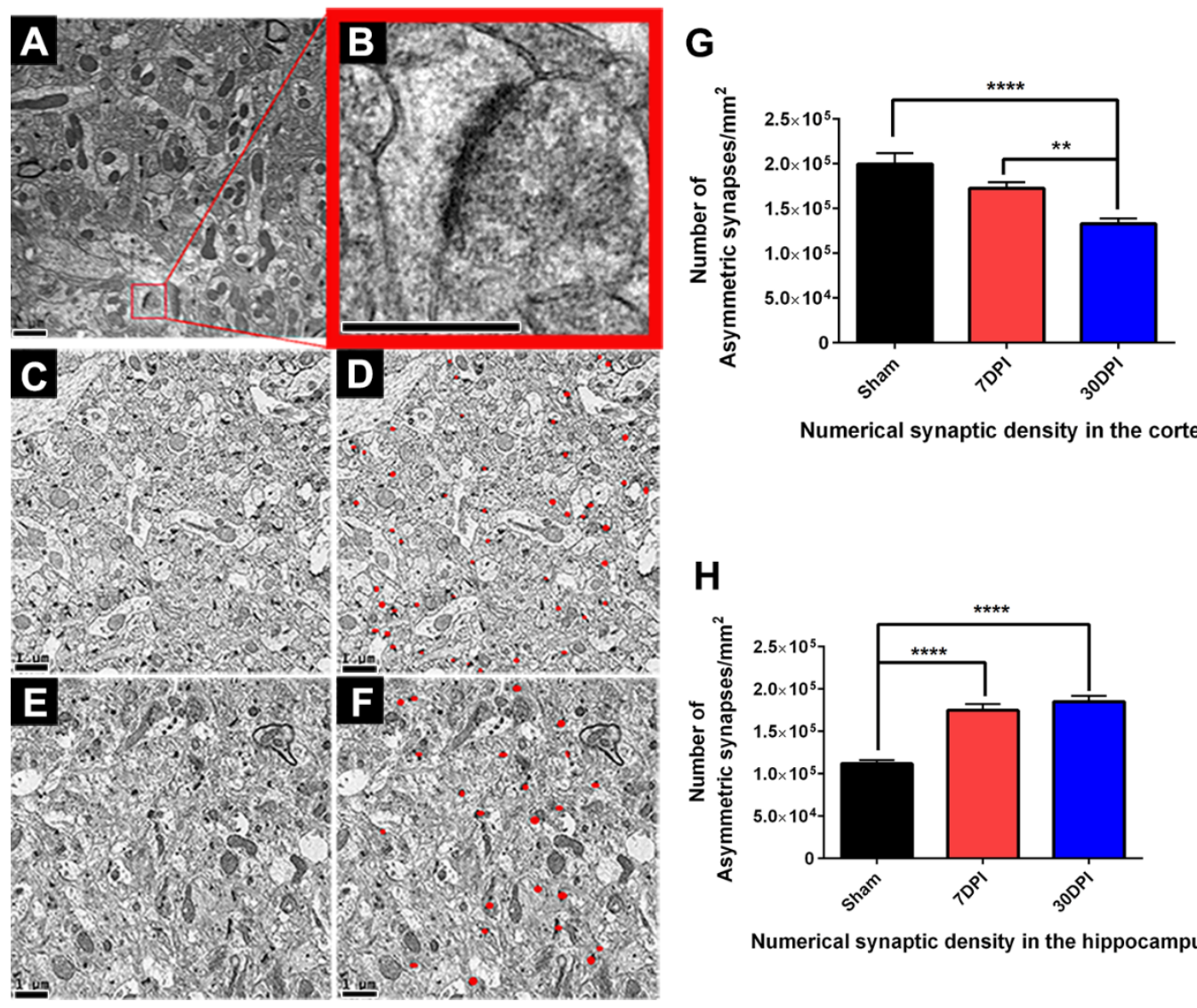

Numerical synaptic density in the cortex

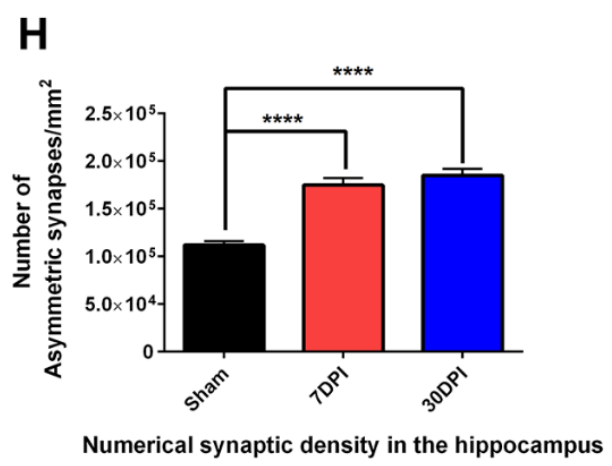

Figure 4.4 Asymmetric synaptic changes after LIB exposure.

(A-B) Low magnification of the normal neuropil in which asymmetric synapses are recognizable by their post synaptic density in sham control group. $(2,000 \mathrm{x}$, scale bar $=1 \mu \mathrm{m})$. (C-D) Representative images of a mouse cortex with red color highlights of asymmetric synapses (D) per area in the sham control $(800 \mathrm{x}$, scale bar $=2 \mu \mathrm{m})$. (E-F) Representative images of a mouse cortex with red highlights of asymmetric synapses (F) per area after LIB $(800 \mathrm{x}$, scale bar $=2 \mu \mathrm{m})$. (G-H) The quantitative comparison of the numerical synaptic density of asymmetric synapses showed significant decreases of asymmetric synapses in the cortex after blast $(G)$, contrasting with significant increases in the hippocampus after blast at 7 and 30 DPI. (One-way ANOVA: followed by Tukey test for multiple comparisons, $* *=\mathrm{P}<0.001, * * * *=\mathrm{P}<0.00001)$ 


\subsubsection{The percentage of the docked vesicles decreased after LIB exposure in the cortex}

To assess the blast effect upon readily releasable synaptic vesicles, we quantified the percentage of docked vesicles for each observed synapse. The percentage of readily releasable vesicles was calculated as the proportion of the total synaptic vesicles located within $50 \mathrm{~nm}$ away from the active zone (Figure 4.5A-D). The percentage of docked vesicles decreased over time to become significantly lower at 30 DPI versus the sham group in the cortex, but not in the hippocampus (ANOVA followed by Tukey multicomparison test, $\mathrm{P}=0.0061$ ) (Figure 4.5E-F).
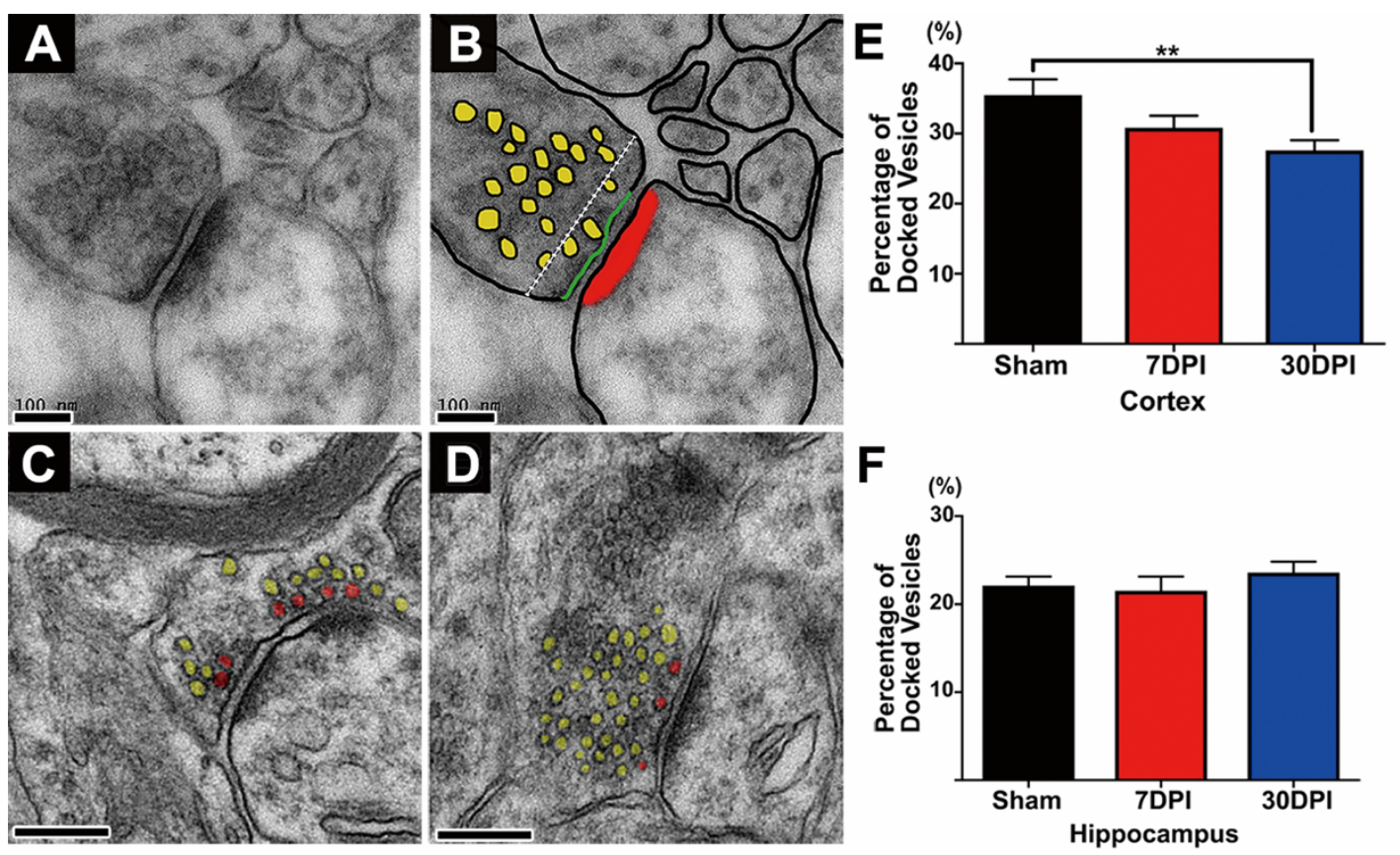

Figure 4.5 Decreased docked vesicles after LIB exposure.

(A-B) Representative TEM images of an asymmetric synapse at high magnification showing features of the synaptic boutons: synaptic vesicles (yellow), active zone (AZ) (green), post synaptic density (PSD) (red). The white line represents $50 \mathrm{~nm}$ distance from 
the active zone $(12,000 \mathrm{x}$, scale bar=100 nm). (C-D) Representative TEM images of cortical region showing synaptic vesicles at the presynaptic terminal in sham (C) and blast (D) groups, respectively $(10,000 \mathrm{x}$, scale bar $=0.2 \mu \mathrm{m})$. The red-colored illustrates docked vesicles while the reserve vesicle pools are yellow-colored. (E-F) One-way ANOVA followed by Tukey test for multiple comparison showed a significant decreased percentage of docked vesicles at 30 DPI in the cortex $(\mathrm{P}=0.009)$ while it remained unchanged in the hippocampus.

\subsubsection{The active zone (AZ) length and the post synaptic density (PSD) thickness were} reorganized distinctively in the cortex and hippocampus after LIB exposure

Next, we examined the quantitative changes at the synaptic interface to determine the $\mathrm{AZ}$ length and the PSD thickness. The AZ length is the critical presynaptic site involved in exocytosis (Figure 4.5A-B) while the PSD thickness is a surrogate of quantitative reorganization of the post synaptic anchorage proteins. As shown in Figure 4.6A, there were no significant differences in the cortex (One-way ANOVA and Tukey multiple comparison test, $\mathrm{P}=0.2056$ ). On the contrary, the PSD thickness (Figure 4.6C) significantly increased at both 7 DPI and 30 DPI (One-way ANOVA, $\mathrm{P}<0.0001$ ). In the hippocampus, the synaptic interface was affected differently. Indeed, at the pre-synaptic membrane, the AZ length decreased significantly at 30 DPI (Figure 4.6B) (One-way ANOVA and Tukey multiple comparison test, $\mathrm{P}=0.0363$ ) while the PSD thickness significantly decreased at 7DPI (Figure 4.6D) (One-way ANOVA and Tukey multiple comparison test, $\mathrm{P}<0.0001)$. 

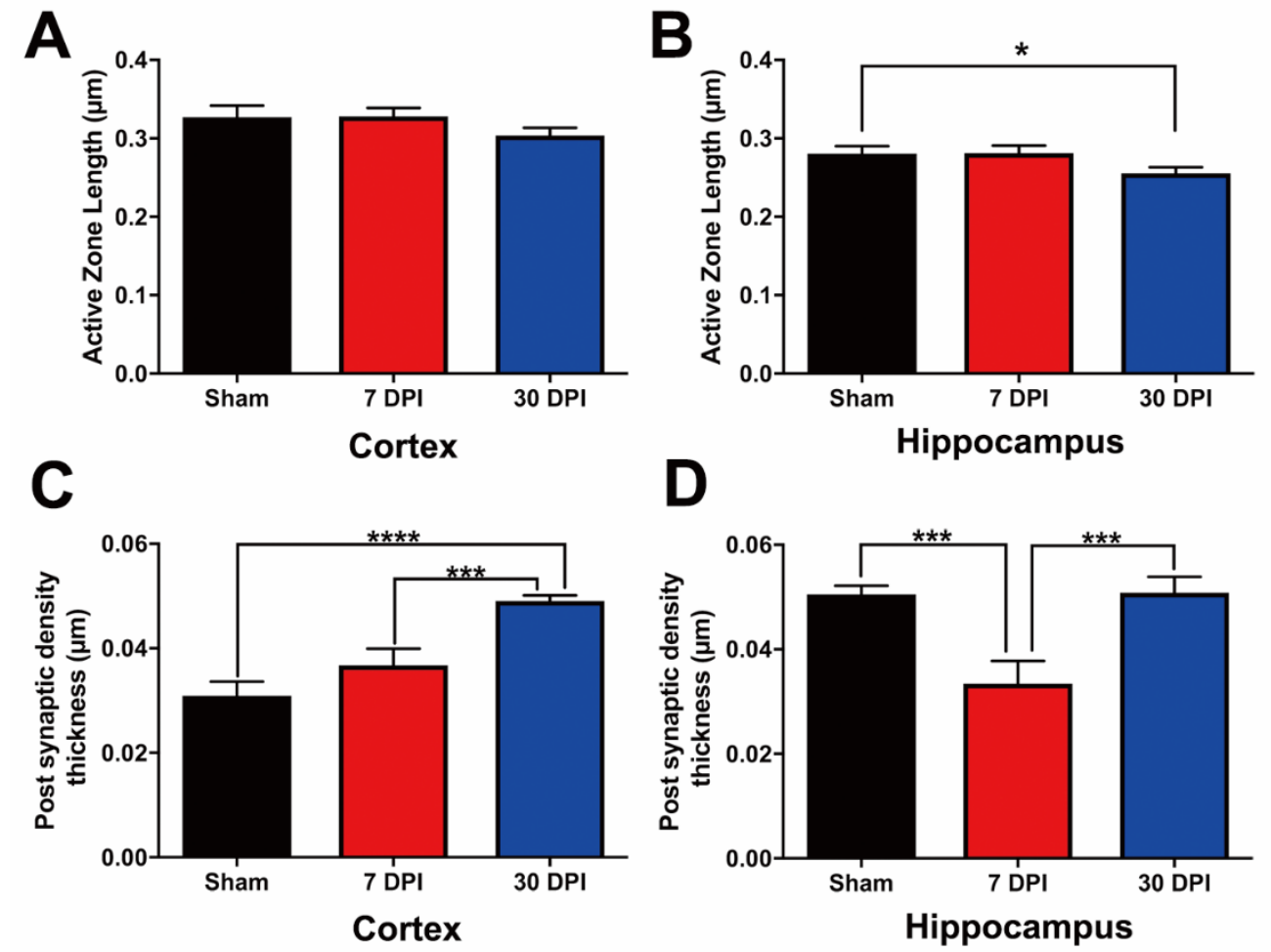

Figure 4.6 Quantitative assessment of the AZ length and the PSD thickness after

\section{LIB exposure.}

(A-B) The AZ length remained unchanged in the cortex while it decreased significantly at 30 DPI in the hippocampus. (C-D) The quantitative comparison of the AZ length and the PSD thickness showed a significant increase of the PSD thickness at both 7DPI and 30 DPI in the cortex while the PSD thickness in the hippocampus CA1 was significantly low only at 7DPI. One -way ANOVA followed by Tukey test for multiple comparison, with * indicating $\mathrm{P}<0.05 ; * * * \mathrm{P}<0.001 ; * * * * \mathrm{P}<0.0001$.

\subsubsection{Pre- and post-synaptic proteins in the cortex increased following LIB exposure}

With further characterization of synaptic proteins level changes, we observed significantly increased PSD95 at 24 hrs and 7 DPI after blast (Figure 4.7A-B). 
Additionally, we identified a significantly elevated synaptophysin level at 7 DPI, with a higher trend at $30 \mathrm{DPI}$, as compared to sham control groups (Figure 4.7C). These findings, along with quantitative ultrastructural findings, suggest that LIB exposure induces synaptic alterations in the brain cortex.
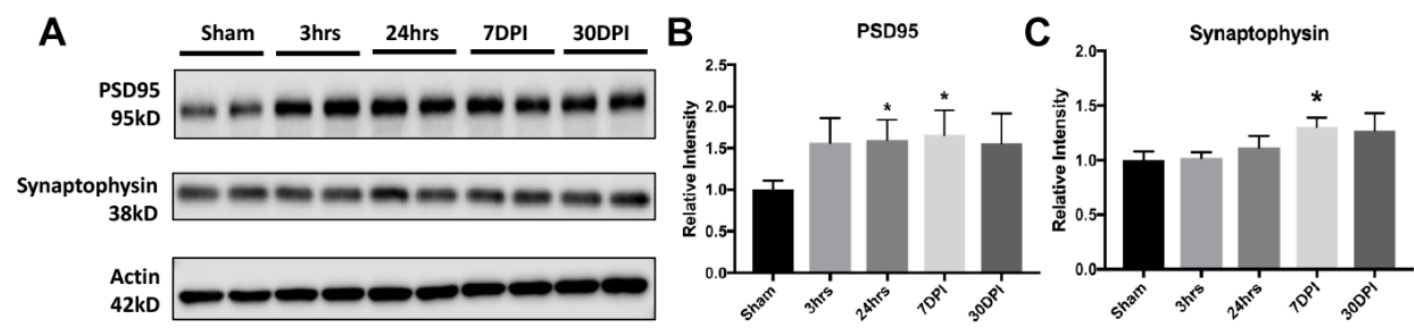

Figure 4.7 LIB induced changes associated with pre- and post-synaptic

\section{dysregulation.}

(A) Western blot and analysis of synaptic markers after low-intensity blast. (B) Postsynaptic marker PSD95 expression was significantly increased after blast as compared to sham at 24 hrs $(\mathrm{P}<0.05)$ and 7 DPI $(\mathrm{P}<0.05)$. (C) Pre-synaptic marker synaptophysin expression was trended higher at 7 and 30 DPI. $\mathrm{N}=4$ for each individual group for comparison. All data are expressed as mean \pm SEM and were analyzed by one-way ANOVA test followed by Tukey test for multiple comparisons. Differences were considered significant at $\mathrm{P}<0.05$ for all analyses, with * indicating $\mathrm{P}<0.05$.

\subsection{DISCUSSION}

The present study shows that single LIB exposure in mice causes ultrastructural changes in brain within multiple subcellular compartments. We found observed ultrastructural degeneration in the perikaryon, axonal microtubules, and synapses. Microtubule alterations were also associated with increased levels of tau/ptau and $A \beta 40 / 42$. We noted 
loss of cortical excitatory (Type I) synapses contrasting with an increase of the hippocampal excitatory synapses. Additionally, ultrastructural synaptic features such as the percentage of docked vesicles, AZ length, and PSD thickness underwent distinct quantifiable variations according to the brain regions following the LIB exposure. These results further demonstrated that specific ultrastructure neuronal changes occur within the brain even in the absence of macroscopic lesions.

\subsubsection{Blast physics underlying observed deleterious effects}

Blast injury consequences have captured widespread public and scientific interests due to its relatively high prevalence within the US military personnel and Veterans. Understanding the physical characteristic of blast wave dynamics and its interactions with the human body is essential for adequate diagnosis and treatment of blast-induced TBI [5]. Synaptic injuries are of particular interest as they related to integrated brain function [30]. In 2012, Kucherov et al. proposed a phonon based physical model to explain the potential mechanisms of blast induced tissue damages [8]. According to this phonon-based theory, detonation of an explosive generates a blast wave that travels through the heterogeneous brain tissue water (70-80\% plus cerebrospinal fluid) at supersonic speed. Nanoscale interaction between the blast shock wave and the heterogeneous brain tissue then induce shear forces and membrane disruptions at ultrastructural levels [8,31]. Blast-induced shear forces may cause transient disconnections and micro-damage to a wide range of cellular and subcellular components including cell membranes, cellular adhesion molecules, cytoskeletons, dendritic spines, 
and synapses. In turn, these injuries may be manifested as transient or later neurocognitive impairments [31].

\subsubsection{Changes in neuronal cell bodies}

A salient finding in the cortical neurons of LIB-exposed animals was a darkened perikaryon with dilated perinuclear spaces, distorted endoplasmic reticulum, and swollen mitochondria. Degenerating neuronal cell bodies in the cortex have been previously reported in animal TBI studies in response to hypoxic events $[11,32]$. The dilated perinuclear spaces, endoplasmic reticulum, and swollen mitochondria suggest impairment of protein synthesis and cellular energetic processes. Stacks of swollen endoplasmic reticulum occurring within the cytoplasm may represent an attempt to sequester excess calcium to exert a protective role $[33,34]$. Although the degenerating neuronal profiles observed were not sufficient for quantification in the present study, a recent report described an increased number of such dark neuronal cell bodies three months after repeated TBI using CCI [11]. No evidence exists yet to determine whether these features of degenerating neuronal cell bodies are reversible or lead to the loss of neurons in animal models of TBI $[31,35]$.

\subsubsection{Cytoskeletal changes}

Microtubule disorganization, the hallmark of axonal breakdown, is associated with impairments in intra-axonal transport, axonal growth, and neurotransmission $[16,36]$. In the present study, we observed several features of cytoskeletal disruption ranging from focal areas of microtubule aggregation to the complex aggregates of microtubules and 
vacuoles. Similar changes also appear in ultrastructural microtubule changes in models of stretched axonal injuries $[16,17]$. The biochemical mechanisms resulting in loss of microtubules are, hypothetically, mediated both by posttraumatic influx of calcium and activation of calmodulin $[37,38]$. This loss results in focal accumulation of membranous organelles in various parts along the length of damaged axons leading to undulation and varicosities, also referred to as "axonal bulbs" [36, 39]. A striking observation in this study was the homogenous electron-dense axoplasmic material also called "dark neurons". Marques et al. reported that the microtubule associated neurofilaments are aggregated but not decomposed at this stage since each of their subunits can be detected by immunostaining techniques for several weeks after injury [40]. Initially, dark axons were believed to be an irreversible state leading to axonal death, however Gallyas et al. challenged this assumption $[17,41]$. Our findings suggest that blast exposure is associated with a significant increase of cytoskeletal damages mainly in the cortex at the acute phase post injury. In a study using a rat model of shock tube driven blast TBI, Baalman et al. found similar microtubule changes [42] in a higher overpressure (74 kPa), whereas the present study with a $46 \mathrm{kPa}$ overpressure indicated that neurofilaments are susceptible to even less intense LIB exposure. Microtubule disorganization affects its associated proteins.

Microtubule-associated protein tau plays a role in the maintenance of cytoskeletal stability, whereas ptau resulting from stresses leads to the destabilization of microtubules. Our data showed a significant rise of total tau at 7 DPI while, ptau, and A $\beta 42$ were significantly elevated at 30 DPI. The increased level of ptau at 30 DPI is consistent with current research on axonal injuries [43]. In a recent multicentric study, Rubeinstein et al. 
suggested the use of ptau and the ratio ptau/total tau as diagnostic and prognostic biomarkers for both acute and chronic TBI. A $\beta 42$ is extensively studied in Alzheimer diseases (AD), has also been described in chronic traumatic encephalopathy [43]. Unlike $\mathrm{AD}$ patients, where $\mathrm{A} \beta$ plaques form over years, accumulation and aggregation of $\mathrm{A} \beta$ in mice occur acutely follow after TBI. Although the mechanism promoting A $\beta$ accumulation remain unclear, studies on repetitive mild TBI posit tau and A $\beta$ accumulation as a direct effect of brain injury [44]. In comparison to the model of repetitive blunt TBI, our study is the first to describe increased levels of total tau, ptau, and/or A $\beta$ following a single LIB exposure. Implying a similar pathophysiology between blast- and blunt-induced TBI, our findings suggest a common initial pathological event or events leading to later neurological deficits. Of note, our results infer that the cortex is susceptible to LIB-induced cytoskeletal damages. Taken together, the present findings show evidence of ultrastructure and protein disorganizations that will affect intra-axonal transport that predicts damage to critical neuronal components, particularly the synaptic interface.

\subsubsection{Blast induced changes in the numerical density of synapses}

The assessment of quantitative change of asymmetric synapses is of importance in various physiological and pathological processes. Synaptic injuries are of particular interest as related to integrated brain function. Their numerical density is a structural parameter or measure of the number of synapses in a defined space. This anatomic measurement directly reflects the excitatory synaptic state and possible inter-neuronal connectivity $[45,46]$. Thus, a reduction of the numerical density of asymmetric synapse 
suggests a loss of excitatory synapses. We found that the density of asymmetric synapses was significantly lower in the cortex of blast animals as compared to sham controls. This finding aligns with earlier studies using various rodent models of brain injury $[47,48]$. The reduced neuronal network may form the anatomical basis for impaired brain functioning. Indeed, the loss of excitatory synapses has been reported in depression models and is similarly documented in neurodegenerative disorders such as AD and in a primate monkey model of Parkinson's disease [49-52]. Additionally, molecular studies have provided supporting evidences of stress induced dendritic atrophy in pyramidal neurons in the cortex $[53,54]$. Synaptic losses may be the secondary effect of diffuse axonal injury (DAI) [55]. Shear and tensile forces associated with the blast TBI may disrupt cell adhesion molecules (CAM) within the synaptic cleft and may play an important part in the reported synaptic losses. Surprisingly, we found in the hippocampus an increased number of asymmetric synapses after blast at both 7DPI and 30DPI. This increase may be a reactive synaptogenic response to the LIB exposure. In fact, in a rat model of CCI, Scheff et al. studied the total number of synapses in CA1 and found an initial loss of $58 \%$ asymmetric synapse at 2 DPI followed by a $168 \%$ increase of asymmetric synapses by 60 DPI. These findings suggest that the hippocampus is capable of initiating a reactive synaptogenic response post injury [48]. We hypothesize that the blast wave travelling through the brain primarily damages cortical structures. The subcortical structures may undergo secondary or possibly reactive changes. We anticipated that such rearrangements potentially would occur at the intra- and interneuronal level, namely at synaptic interfaces. 


\subsubsection{Blast caused synaptic alterations}

Experimental investigations of synaptic injury mechanisms following blast exposure are emerging. Synaptic dysfunction has been reported post-TBI as well as in various neurodegenerative and neuroinflammatory diseases $[30,56]$. To date, these have been observed only in in vitro neuronal cultures and in brain slice preparations of rodents exposed to shock waves [57]. Existing studies of non-blast related TBI reported evidence of alterations in SNARE proteins and reduction of readily releasable synaptic vesicles suggesting an impairment of exocytosis [19]. In the present study, we found a reduced percentage of docked vesicles in the mice cortex after LIB with a significant drop at 30 DPI. We suspected that this finding indicated an impaired release of neurotransmitters into the synaptic cleft [20]. The finding that the active zone length was similar between both Sham and blast animals suggests that a lower percentage of docked vesicles is not reflective of changes in the active zone size. Although theories suggest that the blast induces synaptic disconnection [30], studies showing synaptic ultrastructural abnormalities are scarce, rather functional alterations. In a rat model of CCI, Ojo et al. considered a synapse degenerating when it exhibited very electron-dense pre/post synaptic compartment, swollen pre synaptic process and swollen mitochondria [11]. This is corroborated by studies of mitochondrial respiratory function, indicating that synaptic mitochondria sustain more damage than non-synaptic mitochondria at acute phase post injury $[58,59]$. In the hippocampus, the percentage of docked vesicles was not affected by LIB exposure, suggesting that the hippocampus may undergo a distinct post blast rearrangement. When examining the features of synaptic changes in the cortex, we noticed that the number of excitatory synapses dropped along with the percentage of 
docked vesicles. Yet, the PSD thickness increased. We propose that this increase may be a post synaptic compensatory change that helps to maintain a certain level of synaptic activity by increasing the number of post synaptic receptors. In fact, PSD thickening has been shown to occur in neurons exposed to oxygen-glucose deprivation and also following the activation of glutamate receptors [60]. On the other hand, after LIB exposure, the hippocampus exhibited a significant drop of the PSD thickness at 7 DPI while its synaptogenic activity increased by raising the number of excitatory synapses. However, the percentage of docked vesicles remained invariant after LIB exposure. The absence of qualitative synaptic ultrastructure alterations in our study may be explained by the low intensity of the primary blast wave exposure. However, we found an increased level of synaptophysin and PSD95. Both proteins are markers of synaptic plasticity $[61,62]$. Synaptophysin, a presynaptic protein present in the entire central nervous system has been proven to be involved in synaptic reorganization following TBI. In a mouse model of moderate CCI, Thompson and colleagues also found an increased level of synaptophysin between $24 \mathrm{hrs}$ and 21 DPI [56]. Conversely, losses of synaptophysin were reported in blast exposure on hippocampal slice cultures and in severe CCI $[56,57]$. These findings suggest that the level of synaptophysin may be inversely proportional with the severity of injury. On the post synaptic compartment, PSD95, a guanylate kinase family of scaffolding protein located in the PSD, is implicated in the promotion of synaptic plasticity, neuronal differentiation, and the regulation of ion channels [30]. In moderate CCI in rats, Ansari et al. reported a significant delayed reduction in PSD95 expression in the cortex starting at $48 \mathrm{hrs}$ post injury [63]. These results differ from our blast experiment which indicated a significant increase of PSD95 
level post-LIB exposure. Wakade et al. also found an initial increase of PSD95 in the first week followed by a decrease over time [64]. We suggest that the acute increase of PSD95 may be a compensative mechanism to the loss of asymmetric synapses.

Our results support the concept that primary LIB wave travelling through the brain triggers cascades of biological events causing nanometer scale injuries that translate to neuronal perikaryon, dendritic, axonal, and synaptic abnormalities. In the current study, the observed abnormalities were time- dependent and quantitatively variable within the brain regions. Importantly, we found evidences of synaptic reorganization induced by LIB exposure. The mechanisms of blast induced TBI remain partially understood and the opinions about them are still controversial. Further determinations and measurements are needed to define the critical threshold of blast exposure leading to clinically relevant injuries [5]. A comprehensive three-dimensional characterization of the brain ultrastructural abnormalities in up-scaled gyrencephalic species and primate like skull geometry capable of closely mimicking human injury would be of great help to reach further understanding of this complex form of TBI. Finally, a need exists to translate the findings of acute ultrastructural effects to chronic neuropathology and neurobehavioral abnormalities; for this, well defined preclinical blast models are required. These efforts offer the promise of better understanding of the pathogenesis of TBI and offer new measures to prevent, mitigate or treat the effects of blast injuries. 


\subsection{REFERENCES}

[1] B.D. Owens, J.F. Kragh, Jr., J.C. Wenke, J. Macaitis, C.E. Wade, J.B. Holcomb, Combat wounds in operation Iraqi Freedom and operation Enduring Freedom, J Trauma 64(2) (2008) 295-9.

[2] K.R. Marshall, S.L. Holland, K.S. Meyer, E.M. Martin, M. Wilmore, J.B. Grimes, Mild traumatic brain injury screening, diagnosis, and treatment, Mil Med 177(8 Suppl) (2012) 67-75.

[3] R.G. DePalma, D.G. Burris, H.R. Champion, M.J. Hodgson, Blast injuries, N Engl J Med 352(13) (2005) 1335-1342.

[4] H.L. Song, J.K. Cui, A. Simonyi, C.E. Johnson, G.K. Hubler, R.G. DePalma, Z.Z. Gu, Linking blast physics to biological outcomes in mild traumatic brain injury: Narrative review and preliminary report of an open-field blast model, Behav Brain Res 340 (2018) $147-158$.

[5] I. Cernak, D.G. Stein, G.A. Elder, S. Ahlers, K. Curley, R.G. DePalma, J. Duda, M. Ikonomovic, G.L. Iverson, F. Kobeissy, V.E. Koliatsos, M.J. Leggieri, A.M. Pacifico, D.H. Smith, R. Swanson, F.J. Thompson, F.C. Tortella, Preclinical modelling of militarily relevant traumatic brain injuries: Challenges and recommendations for future directions, Brain Injury 31(9) (2017) 1168-1176.

[6] H. Song, L.M. Konan, J. Cui, C.E. Johnson, M. Langenderfer, D. Grant, T. Ndam, A. Simonyi, T. White, U. Demirci, D.R. Mott, D. Schwer, G.K. Hubler, I. Cernak, R.G. DePalma, Z. Gu, Ultrastructural brain abnormalities and associated behavioral changes in mice after low-intensity blast exposure, Behav Brain Res 347 (2018) 148-157. 
[7] H. Song, L.M. Konan, J. Cui, C.E. Johnson, G.K. Hubler, R.G. DePalma, Z. Gu, Nanometer ultrastructural brain damage following low intensity primary blast wave exposure, Neural Regen Res 13(9) (2018) 1516.

[8] Y. Kucherov, G.K. Hubler, R.G. DePalma, Blast induced mild traumatic brain injury/concussion: A physical analysis, J Appl Phys 112(10) (2012) 104701.

[9] Q. Wu, S.X. Xia, Q.Q. Li, Y. Gao, X. Shen, L. Ma, M.Y. Zhang, T. Wang, Y.S. Li, Z.F. Wang, C.L. Luo, L.Y. Tao, Mitochondrial division inhibitor 1 (Mdivi-1) offers neuroprotection through diminishing cell death and improving functional outcome in a mouse model of traumatic brain injury, Brain Res 1630 (2016) 134-143.

[10] T. Taib, C. Leconte, J. Van Steenwinckel, A.H. Cho, B. Palmier, E. Torsello, R. Lai Kuen, S. Onyeomah, K. Ecomard, C. Benedetto, B. Coqueran, A.C. Novak, E. Deou, M. Plotkine, P. Gressens, C. Marchand-Leroux, V.C. Besson, Neuroinflammation, myelin and behavior: Temporal patterns following mild traumatic brain injury in mice, PLoS One 12(9) (2017) e0184811.

[11] J.O. Ojo, C. Bachmeier, B.C. Mouzon, R. Tzekov, M. Mullan, H. Davies, M.G. Stewart, F. Crawford, Ultrastructural Changes in the White and Gray Matter of Mice at Chronic Time Points After Repeated Concussive Head Injury, J Neuropathol Exp Neurol 74(10) (2015) 1012-35.

[12] E. Fievisohn, Z. Bailey, A. Guettler, P. VandeVord, Primary Blast Brain Injury Mechanisms: Current Knowledge, Limitations, and Future Directions, J Biomech Eng 140(2) (2018) 020806.

[13] X. Du, M.B. West, W. Cheng, D.L. Ewert, W. Li, D. Saunders, R.A. Towner, R.A. Floyd, R.D. Kopke, Ameliorative Effects of Antioxidants on the Hippocampal 
Accumulation of Pathologic Tau in a Rat Model of Blast-Induced Traumatic Brain Injury, Oxidative medicine and cellular longevity 2016 (2016) 4159357.

[14] C.M. Parisian, G. Georgevitch, B.A. Bahr, Military blast-induced synaptic changes with distinct vulnerability may explain behavioral alterations in the absence of obvious brain damage, J Nat Sci 3(7) (2017).

[15] I. Cernak, Z. Wang, J. Jiang, X. Bian, J. Savic, Ultrastructural and functional characteristics of blast injury-induced neurotrauma, J Trauma Acute Care Surg 50(4) (2001) 695-706.

[16] A.J. Fournier, J.D. Hogan, L. Rajbhandari, S. Shrestha, A. Venkatesan, K.T. Ramesh, Changes in Neurofilament and Microtubule Distribution following Focal Axon Compression, PLoS One 10(6) (2015) e0131617.

[17] F. Gallyas, J. Pal, O. Farkas, T. Doczi, The fate of axons subjected to traumatic ultrastructural (neurofilament) compaction: an electron-microscopic study, Acta Neuropathol 111(3) (2006) 229-37.

[18] E.G. Gray, Electron Microscopy of Synaptic Contacts on Dendrite Spines of the Cerebral Cortex, Nature 183(4675) (1959) 1592-1593.

[19] S.W. Carlson, H. Yan, M. Ma, Y. Li, J. Henchir, C.E. Dixon, Traumatic Brain Injury Impairs Soluble N-Ethylmaleimide-Sensitive Factor Attachment Protein Receptor Complex Formation and Alters Synaptic Vesicle Distribution in the Hippocampus, J Neurotrauma 33(1) (2016) 113-21. [20] L.D. Pozzo-Miller, W. Gottschalk, L. Zhang, K. McDermott, J. Du, R. Gopalakrishnan, C. Oho, Z.H. Sheng, B. Lu, Impairments in high-frequency 
transmission, synaptic vesicle docking, and synaptic protein distribution in the hippocampus of BDNF knockout mice, J Neurosci 19(12) (1999) 4972-4983.

[21] K.M. Harris, R.J. Weinberg, Ultrastructure of synapses in the mammalian brain, Cold Spring Harb Perspect Biol 4(5) (2012) a005587.

[22] N.E. Reist, J. Buchanan, J. Li, A. DiAntonio, E.M. Buxton, T.L. Schwarz, Morphologically Docked Synaptic Vesicles Are Reduced insynaptotagmin Mutants of Drosophila, J Neurosci 18(19) (1998) 7662-7673.

[23] Z. Qu, F.J. Meng, R.D. Bomgarden, R.I. Viner, J.L. Li, J.C. Rogers, J.L. Cheng, C.M. Greenlief, J.K. Cui, D.B. Lubahn, G.Y. Sun, Z.Z. Gu, Proteomic Quantification and Site-Mapping of S-Nitrosylated Proteins Using Isobaric iodoTMT Reagents, J Proteome Res 13(7) (2014) 3200-3211.

[24] M. Chen, H. Song, J. Cui, C. Johnson, G. Hubler, R.G. DePalma, Z. Gu, X.

Weiming, Proteomic profiling of mouse brains exposed to blast-induced mild traumatic brain injury reveals changes in axonal proteins and phosphorylated tau, J Alzheimers Dis Pre-press(Pre-press) (2018) 1-23.

[25] S.S. Jafari, W.L. Maxwell, M. Neilson, D.I. Graham, Axonal cytoskeletal changes after non-disruptive axonal injury, J Neurocytol 26(4) (1997) 207-221.

[26] V.E. Johnson, W. Stewart, J.D. Arena, D.H. Smith, Traumatic brain injury as a trigger of neurodegeneration, Neurodegener Dis, Springer2017, pp. 383-400.

[27] B.R. Huber, J.S. Meabon, T.J. Martin, P.D. Mourad, R. Bennett, B.C. Kraemer, I. Cernak, E.C. Petrie, M.J. Emery, E.R. Swenson, C. Mayer, E. Mehic, E.R. Peskind, D.G. Cook, Blast exposure causes early and persistent aberrant phospho- and cleaved-tau 
expression in a murine model of mild blast-induced traumatic brain injury, J Alzheimers Dis 37(2) (2013) 309-23.

[28] T.L. Spires-Jones, B.T. Hyman, The Intersection of Amyloid Beta and Tau at Synapses in Alzheimer's Disease, Neuron 82(4) (2014) 756-771.

[29] S.N. Burke, C.A. Barnes, Neural plasticity in the ageing brain, Nature Rev Neurosci 7(1) (2006) 30.

[30] L. Merlo, F. Cimino, F.F. Angileri, D. La Torre, A. Conti, S.M. Cardali, A. Saija, A. Germano, Alteration in synaptic junction proteins following traumatic brain injury, $\mathrm{J}$ Neurotrauma 31(16) (2014) 1375-85.

[31] M.A.G. Sosa, R. De Gasperi, A.J. Paulino, P.E. Pricop, M.C. Shaughness, E. Maudlin-Jeronimo, A.A. Hall, W.G.M. Janssen, F.J. Yuk, N.P. Dorr, D.L. Dickstein, R.M. McCarron, M. Chavko, P.R. Hof, S.T. Ahlers, G.A. Elder, Blast overpressure induces shear-related injuries in the brain of rats exposed to a mild traumatic brain injury, Acta Neuropathol Com 1 (2013) 51.

[32] J. Lu, K.C. Ng, G. Ling, J. Wu, D.J. Poon, E.M. Kan, M.H. Tan, Y.J. Wu, P. Li, S. Moochhala, E. Yap, L.K. Lee, M. Teo, I.B. Yeh, D.M. Sergio, F. Chua, S.D. Kumar, E.A. Ling, Effect of blast exposure on the brain structure and cognition in Macaca fascicularis, J Neurotrauma 29(7) (2012) 1434-54.

[33] S. Orrenius, B. Zhivotovsky, P. Nicotera, Calcium: Regulation of cell death: the calcium-apoptosis link, Nat Rev Mol Cell Biol 4(7) (2003) 552.

[34] J.H. Lin, P. Walter, T.S.B. Yen, Endoplasmic reticulum stress in disease pathogenesis, Annu Rev Pathol 3 (2008) 399-425. 
[35] B.C. Mouzon, C. Bachmeier, A. Ferro, J.O. Ojo, G. Crynen, C.M. Acker, P. Davies, M. Mullan, W. Stewart, F. Crawford, Chronic neuropathological and neurobehavioral changes in a repetitive mild traumatic brain injury model, Ann Neurol 75(2) (2014) 24154.

[36] M.D. Tang-Schomer, A.R. Patel, P.W. Baas, D.H. Smith, Mechanical breaking of microtubules in axons during dynamic stretch injury underlies delayed elasticity, microtubule disassembly, and axon degeneration, Faseb J 24(5) (2010) 1401-1410. [37] J. Ma, K. Zhang, Z. Wang, G. Chen, Progress of Research on Diffuse Axonal Injury after Traumatic Brain Injury, Neural plasticity 2016 (2016) 9746313.

[38] W.L. Maxwell, D.I. Graham, Loss of axonal microtubules and neurofilaments after stretch-injury to guinea pig optic nerve fibers, J Neurotrauma 14(9) (1997) 603-14. [39] M.D. Tang-Schomer, V.E. Johnson, P.W. Baas, W. Stewart, D.H. Smith, Partial interruption of axonal transport due to microtubule breakage accounts for the formation of periodic varicosities after traumatic axonal injury, Exp Neurol 233(1) (2012) 364-72. [40] S.A. Marques, M. Taffarel, A.M.B. Martinez, Participation of neurofilament proteins in axonal dark degeneration of rat's optic nerves, Brain Res 969(1-2) (2003) 1-13. [41] A. Csordas, M. Mazlo, F. Gallyas, Recovery versus death of "dark" (compacted) neurons in non-impaired parenchymal environment: light and electron microscopic observations, Acta Neuropathol 106(1) (2003) 37-49.

[42] K.L. Baalman, R.J. Cotton, S.N. Rasband, M.N. Rasband, Blast wave exposure impairs memory and decreases axon initial segment length, J Neurotrauma 30(9) (2013) $741-51$. 
[43] R. Rubenstein, B. Chang, J.K. Yue, A. Chiu, E.A. Winkler, A.M. Puccio, R. DiazArrastia, E.L. Yuh, P. Mukherjee, A.B. Valadka, Comparing plasma phospho tau, total tau, and phospho tau-total tau ratio as acute and chronic traumatic brain injury biomarkers, JAMA Neurol 74(9) (2017) 1063-1072.

[44] G. Edwards III, I. Moreno-Gonzalez, C. Soto, Amyloid-beta and tau pathology following repetitive mild traumatic brain injury, Biochem Biophys Res Commun 483(4) (2017) 1137-1142.

[45] S. Chaudhury, T.C. Nag, S. Wadhwa, Effect of prenatal auditory stimulation on numerical synaptic density and mean synaptic height in the posthatch Day 1 chick hippocampus, Synapse (New York, N.Y.) 63(2) (2009) 152-9.

[46] S.W. Scheff, D.A. Price, Alzheimer's disease-related alterations in synaptic density: neocortex and hippocampus, J Alzheimers Dis 9(3 Suppl) (2006) 101-15.

[47] V. Semchenko, N. Bogolepov, S. Stepanov, S. Maksimishin, A. Khizhnyak, Synaptic plasticity of the neocortex of white rats with diffuse-focal brain injuries, Neurosci Behav Physiol 36(6) (2006) 613-618.

[48] S.W. Scheff, D.A. Price, R.R. Hicks, S.A. Baldwin, S. Robinson, C. Brackney, Synaptogenesis in the hippocampal CA1 field following traumatic brain injury, J Neurotrauma 22(7) (2005) 719-732.

[49] D. Csabai, O. Wiborg, B. Czeh, Reduced Synapse and Axon Numbers in the Prefrontal Cortex of Rats Subjected to a Chronic Stress Model for Depression, Front Cell Neurosci 12(24) (2018) 24.

[50] W. Timmermans, H. Xiong, C.C. Hoogenraad, H.J. Krugers, Stress and excitatory synapses: from health to disease, Neuroscience 248 (2013) 626-36. 
[51] J.D. Elsworth, C. Leranth, D.E. Redmond, R.H. Roth, Loss of asymmetric spine synapses in prefrontal cortex of motor-asymptomatic, dopamine-depleted, cognitively impaired MPTP-treated monkeys, Int J Neuropsychoph 16(4) (2013) 905-912.

[52] R. Clare, V.G. King, M. Wirenfeldt, H.V. Vinters, Synapse loss in dementias, J Neurosci Res 88(10) (2010) 2083-90.

[53] J.J. Radley, A.B. Rocher, M. Miller, W.G. Janssen, C. Liston, P.R. Hof, B.S. McEwen, J.H. Morrison, Repeated stress induces dendritic spine loss in the rat medial prefrontal cortex, Cereb Cortex 16(3) (2006) 313-20.

[54] J.J. Radley, H.M. Sisti, J. Hao, A.B. Rocher, T. McCall, P.R. Hof, B.S. McEwen, J.H. Morrison, Chronic behavioral stress induces apical dendritic reorganization in pyramidal neurons of the medial prefrontal cortex, Neuroscience 125(1) (2004) 1-6. [55] X. Gao, P. Deng, Z.C. Xu, J.H. Chen, Moderate Traumatic Brain Injury Causes Acute Dendritic and Synaptic Degeneration in the Hippocampal Dentate Gyrus, PloS One 6(9) (2011) e24566.

[56] S.N. Thompson, T.R. Gibson, B.M. Thompson, Y. Deng, E.D. Hall, Relationship of calpain-mediated proteolysis to the expression of axonal and synaptic plasticity markers following traumatic brain injury in mice, Exp Neurol 201(1) (2006) 253-65.

[57] M. Smith, T. Piehler, R. Benjamin, K.L. Farizatto, M.C. Pait, M.F. Almeida, V.V. Ghukasyan, B.A. Bahr, Blast waves from detonated military explosive reduce GluR1 and synaptophysin levels in hippocampal slice cultures, Exp Neurol 286 (2016) 107-115. [58] J.R. Kulbe, R.L. Hill, I.N. Singh, J.A. Wang, E.D. Hall, Synaptic Mitochondria Sustain More Damage than Non-Synaptic Mitochondria after Traumatic Brain Injury and Are Protected by Cyclosporine A, J Neurotrauma 34(7) (2017) 1291-1301. 
[59] H. Chen, Y.L. Chan, L.T. Nguyen, Y. Mao, A. de Rosa, I.T. Beh, C. Chee, B.

Oliver, G. Herok, S. Saad, C. Gorrie, Moderate traumatic brain injury is linked to acute behaviour deficits and long term mitochondrial alterations, Clinical and experimental pharmacology \& physiology 43(11) (2016) 1107-1114.

[60] Y. Fukunaga, E. Nakajima, E. Hatano, S. Itoh, Y. Kashino, A. Miyazawa, Activation of NMDA receptors thickens the postsynaptic density via proteolysis, Neurosci Res 101 (2015) 6-14.

[61] A.E.-D. El-Husseini, E. Schnell, D.M. Chetkovich, R.A. Nicoll, D.S. Bredt, PSD-95 involvement in maturation of excitatory synapses, Science 290(5495) (2000) 1364-1368. [62] R. Janz, T.C. Südhof, R.E. Hammer, V. Unni, S.A. Siegelbaum, V.Y. Bolshakov, Essential roles in synaptic plasticity for synaptogyrin I and synaptophysin I, Neuron 24(3) (1999) 687-700.

[63] M.A. Ansari, K.N. Roberts, S.W. Scheff, A time course of contusion-induced oxidative stress and synaptic proteins in cortex in a rat model of TBI, J Neurotrauma $25(5)(2008) 513-526$.

[64] C. Wakade, S. Sukumari-Ramesh, M.D. Laird, K.M. Dhandapani, J.R. Vender, Delayed reduction in hippocampal postsynaptic density protein-95 expression temporally correlates with cognitive dysfunction following controlled cortical impact in mice, $\mathrm{J}$ Neurosurg 113(6) (2010) 1195-1201. 


\section{CHAPTER 5 PROTEOMIC ANALYSIS AND \\ BIOCHEMICAL CORRELATES OF MITOCHONDRIAL \\ DYSFUNCTION FOLLOWING LOW-INTENSITY}

PRIMARY BLAST EXPOSURE

\subsection{ABSTRACT}

Service members during military actions or combat training are frequently exposed to primary blasts by weaponry. Most studies have investigated moderate or severe brain injuries from blasts generating overpressures over $100-\mathrm{kPa}$, while understanding the pathophysiology of low-intensity blast (LIB)-induced mild traumatic brain injury (mTBI) leading to neurological deficits remains elusive. Our recent studies, using an open-field LIB-induced mTBI mouse model with a peak overpressure at $46.6-\mathrm{kPa}$, demonstrated behavioral impairments and brain nanoscale damages, notably mitochondrial and axonal ultrastructural changes. In this study, we used tandem mass tagged (TMT) quantitative proteomics and bioinformatics analysis to seek insights into the molecular mechanisms underlying ultrastructural pathology. Changes in global- and phospho-proteomes were determined at 3 and 24 hours, 7 and 30 days post injury (DPI), in order to investigate the biochemical and molecular correlates of mitochondrial dysfunction. Results showed striking dynamic changes in a total of 2216 proteins and 459 phosphorylated proteins at vary time points after blast. Disruption of key canonical pathways included evidence of mitochondrial dysfunction, oxidative stress, axonal/cytoskeletal/synaptic dysregulation, and neurodegeneration. Bioinformatic analysis identified blast induced trends in networks 
related to cellular growth/development/movement/assembly and cell-to-cell signaling interactions. With observations of proteomic changes, we found LIB-induced oxidative stress associated with mitochondrial dysfunction mainly at 7 and 30 DPI. These dysfunctions included impaired fission-fusion dynamics, diminished mitophagy, decreased oxidative phosphorylation, and compensated respiration-relevant enzyme activities. Insights on the early pathogenesis of primary LIB-induced brain damage provide a template for further characterization of its chronic effects, identification of potential biomarkers and targets for intervention.

\subsection{INTRODUCTION}

Military personnel frequently exposed to blast waves from explosive weaponry may develop traumatic brain injuries (TBI) of varying severity. Department of Defense and Veterans Administration (DoD / VA) reports indicated that the majority (82.3\%) of blastinduced neurotrauma are mild TBI (mTBI) / concussion, as the "signature wound" of current military conflicts. Subjects with mTBI often do not show detectable signals using conventional imaging techniques and thus missing the opportunities for early diagnosis and treatment $[1,2]$. Nevertheless, these subjects are at risk for development of posttraumatic stress disorder, as well as neurodegenerative disorders that result in life-long disabilities [3, 4]. While studies have provided insights into TBI mechanisms with moderate- to high-intensity blast exposures over 100-kPa peak overpressure, an urgent need still exists to understand the pathobiology underlying the impact of primary lowintensity blast (LIB)-induced brain injury. 
We have recently established an open-field primary LIB murine model to simulate theater combat and military training scenarios [5-8]. With exposure to a magnitude of $46.6 \mathrm{kPa}$ and maximum impulse of $8.7 \mathrm{PSI}(\mathrm{aka} 60 \mathrm{kPa}) \times \mathrm{ms}$ blast, in the absence of head motion, animals experienced no mortality nor gross bodily injuries. Nevertheless, despite the absence of macroscopic changes or cell necrosis, behavioral deficits and ultrastructural damage including mitochondrial abnormalities and myelinated axonal injury were observed [5]. Oxidative stress and mitochondrial dysfunction may decrease mitochondrial fission-fusion activities, and compromise mitophagy and late cellular impairment. Similar signals of mitochondrial dysfunction are found in the pathogenesis of chronic neurodegenerative diseases associated with moderate- or severe TBI [9-12]. However, the underlying mechanisms in LIB exposure leading to the sustained mitochondrial ultrastructural changes coupled with axonal injury in neuronal cells remain elusive. In the present study, we used systems biology analysis of proteomic profiles to delineate mechanisms associated with mitochondrial ultrastructural changes coupled with axonal injury caused by LIB exposure.

Mass spectrometry (MS)-based proteomics is a specific and sensitive tool to determine protein abundance in brain tissues and to predict biochemical responses [1315]. Quantitative proteomics coupled with bioinformatics have aided the discovery of key proteins, including glial fibrillary acidic protein (GFAP) and ubiquitin carboxyl-terminal hydrolase isozyme L1 (UCH-L1), with severe impact or penetrating TBI [16-19]. However, the molecular signatures after LIB exposure remain undefined. In this study, we employed tandem mass tag (TMT) 10-plex peptidyl labeling coupled with liquid chromatography tandem mass spectrometry (LC-MS/MS) to investigate global- and 
phospho- proteomes at different times ( $3 \mathrm{hrs}, 24 \mathrm{hrs}, 7 \mathrm{DPI}$, and $30 \mathrm{DPI})$ after LIB exposure.

Results of this study provide information on specific proteins, signaling pathways / networks, and potential mechanisms related to oxidative stress associated mitochondrial dysfunction and dysregulation of axonal / cytoskeletal / synaptic proteins. These insights regarding early effects on mitochondria can offer new strategies for prevention and treatment of primary LIB-induced brain injury.

\subsection{METHOD}

\subsubsection{Materials}

Chemicals were purchased from Sigma-Aldrich (St. Louis, MO, USA). Anesthesia:

Ketamine hydrochloride injection (100mg/mL, 1044102, Henry Schein, Melville, NY, USA), and Xylazine hydrochloride injection (100mg/mL, 13985-704-10, Vet One, Boise, ID, USA). Reagents for BCA protein assay and sample preparation for LC-MS/MS analysis were purchased from Thermo Scientific (Rockford, IL, USA). TMT reagent 10plex kits were bought commercially (Thermo Scientific Pierce, Rockford, IL, USA).

\subsubsection{Experimental design and statistical rationale}

The experimental design incorporated 4 biological replicates for each group (total 5 groups: sham control, 3 and 24 hrs, 7 and 30 DPI after blast), across two independent TMT 10-plex tagging experiments to examine brain cortex. In order to perform robust quantitative analysis between the two independent TMT 10-plex tagging experiments, we pooled two biological replicates from the sham group together as an index for two TMT 
tagging cross-batch analysis. All experimental groups in the studies were handled in a randomized, double-blinded manner. For quantification purposes, all proteomics data were normalized across all proteins within each 10-plex batch to eliminate the batch effect. Welch's test and t-distribution were performed with R function "t.test" for proteomics data and other statistical comparisons were made by student's t test for two groups and by one-way ANOVA test followed by Tukey test for multiple comparisons. GraphPad Prism (version 6 for Windows; GraphPad Software) were used to calculate the $\mathrm{P}$ values. The relative protein abundance ratios (fold changes) between blast and sham groups were calculated. As previously described, the changes in protein levels were considered significant if fold change was $>1.0$ (up-regulated) or $<1.0$ (down-regulated), and the $\mathrm{P}$ value less than 0.05 in two independent experiments [20]. The threshold of this open-field LIB was determined based upon peak overpressure (46.6 $\mathrm{kPa})$ as compared to literature reports (majority over $100 \mathrm{kPa}$ ) [6, 21], and our previous pathological observations demonstrating the absence of macroscopic damage or necrosis in the presence of nanoscale ultrastructural injuries [5].

\subsubsection{Animals and open-field blast setting}

The protocol for this study is described previously [5]. Twenty male C57BL/6J mice (The Jackson Laboratory, Bar Harbor, ME) at 2 months of age were housed with a 12-h light/dark cycle (lights on at 7:00 AM) and given unrestricted access to food and water. Four mice were put in one cage. A cotton pad and an environmental enrichment toy (small plastic house) were placed in each cage. All animals were observed for good health before, during, and after testing. All experimental groups in the studies were 
handled in a randomized, double-blinded manner. All procedures were carried out in accordance to the University of Missouri approved protocols for the Care and Use of Laboratory Animals and the ARRIVE guidelines.

Based on the blast exposure setting, animals were randomly grouped as the 3-m $(\mathrm{n}=16)$ blast, and sham control $(\mathrm{n}=4)$. Animals with 3 -m blast were randomly grouped into 4 groups as $3 \mathrm{hrs}(\mathrm{n}=4), 24 \mathrm{hrs}(\mathrm{n}=4), 7 \mathrm{DPI}(\mathrm{n}=4)$, and $30 \mathrm{DPI}(\mathrm{n}=4)$. Mice were anesthetized with intraperitoneal (i.p.) injection of $5 \mu \mathrm{L} / \mathrm{g}$ body weight of Ketamine/Xylazine mixture (25 mg/mL Ketamine and $1.25 \mathrm{mg} / \mathrm{mL}$ Xylazine). Sham group underwent the identical anesthesia procedures only without blast exposure. Mice were placed in the prone position at the 3 -m platforms away from the $350 \mathrm{~g} \mathrm{C} 4$. No restrain was applied to the animals. Following blast wave exposure, animals were returned to the original cage. After recovery from anesthesia, mice were able to spontaneously move and continuously being monitored for at least 15-30 min. Animals were allowed access to food and water ad libitum before and after blast exposure.

\subsubsection{Protein sample preparation, isobaric TMT 10-plex labeling, IMAC enrichment, LC-MS/MS analysis, and protein identification}

After blast exposure, cerebral cortices were dissected from the immediately euthanized mouse brains as described previously [5, 6]. To lyse each brain tissue specimen (100 mg), $600 \mu \mathrm{L}$ of sample buffer (2\% SDS, $0.5 \mathrm{M}$ tetraethylammonium bicarbonate (TEAB), protease inhibitor cocktail was added and the specimen homogenized by TissueLyser LT (Qiagen, Valencia, CA). Tissue homogenates were centrifuged at 17,000 × g, for $20 \mathrm{~min}$ at $4{ }^{\circ} \mathrm{C}$. The supernatant was transferred into a new vial for protein concentration 
measurement by BCA assay. Preparation of tryptic peptides for TMT 10-plex labelling was carried out according to manufacturer's instructions. Briefly, $100 \mu \mathrm{g}$ protein from each sample was transferred into a new vial and adjusted to a final volume of $100 \mu \mathrm{L}$ with TEAB and reduced with Tris (2-carboxyethyl) phosphine hydrochloride (TCEP) at $55{ }^{\circ} \mathrm{C}$ for $1 \mathrm{hr}$, and then alkylated with iodoacetamide for 30 minutes in the dark. Methanol-chloroform precipitation was performed prior to protease digestion. In brief, four volumes of methanol were added to each sample and vortexed, followed by adding one part chloroform and three parts water to the sample and vortexed. The sample was centrifuged at 14,000 $\mathrm{g}$ for $4 \mathrm{~min}$ at room temperature and the aqueous phase was removed. The organic phases with protein precipitate at the surface was subsequently washed twice with four parts methanol, centrifuged and subsequently supernatant being removed. After air-drying, precipitated protein pellets were re-suspended with $100 \mu \mathrm{L}$ of $50 \mathrm{mM}$ TEAB and digested with trypsin overnight at $37^{\circ} \mathrm{C}$.

Tagging with TMT multiplex reagents enables relative quantitation of proteins present in multiple samples by labeling peptides with isobaric stable isotope tags. In this study, TMT 10-plex reagents were used for labeling of ten samples (blast samples and controls) and analyzed simultaneously, in order to avoid run-to-run variation. Tryptic digested peptides from brain samples were labeled with TMT 10-plex reagents (Thermo Fisher Scientific) according to manufacturer's instructions. Briefly, the TMT reagents $(0.8 \mathrm{mg})$ were dissolved in $41 \mu \mathrm{L}$ of anhydrous acetonitrile. Aliquots of $95 \mu \mathrm{L}$ of the trypsin-digested peptides from cortical tissue samples were incubated with TMT reagents for $1 \mathrm{hr}$ at room temperature. The reactions were quenched by $8 \mu \mathrm{L}$ of $5 \%$ hydroxylamine solution and reacted for $15 \mathrm{~min}$. The combined TMT labelled samples were dried under 
SpeedVac, and then reconstituted by dilute trifluoroacetic acid solution followed by desalting by Oasis HLB 96-well $\mu$ Elutionplate (Waters) prior to LC-MS/MS analysis. Immobilized metal affinity chromatography (IMAC) was used as an affinity enrichment technique for the preparation of phosphopeptides for mass spectrometric analysis and sequencing as previously described [22]. Briefly, Pierce ferric nitrilotriacetate (Fe-NTA) Phosphopeptide Enrichment Kit (Thermo Fisher Scientific \#88300) was used following the manufacture's protocol. This kit enables fast and efficient enrichment of phosphorylated peptides. Each spin column included in the kit contains phosphopeptide-specific resin that offers excellent binding and recovery properties for enriching phosphopeptides. Each trypsin-digested dried sample was resuspended in $200 \mu \mathrm{L}$ of binding buffer and was added to a Fe-NTA spin column followed by incubation for $20 \mathrm{~min}$ with a slow end-over-end rotation at room temperature. Then, the Fe-NTA spin column was centrifuged at $1,000 \times \mathrm{g}$ for $1 \mathrm{~min}$ and washed with wash buffer. The eluted phosphopeptides were applied to a Pierce Graphite Spin column (Thermo Fisher Scientific \#88302), which enables fast and efficient capture, concentration, desalting and elution of hydrophilic peptides. All eluents were quantitated, analyzed, and the phosphorylation sites were localized.

We performed proteomic profiling of global and phospho-proteomes using the established workflow for MS analysis. LC-MS/MS was performed on a Q Exactive Hybrid Quadrupole-Orbitrap Mass Spectrometer (Thermo Fisher Scientific) coupled with a Dionex ultimate 3000 HPLC system equipped with a nano-ES ion source [23]. The TMT labelled peptides were separated on a C18 reverse-phase capillary column (PepMap, $75 \mu \mathrm{m}$ x $150 \mathrm{~mm}$, Thermo Fisher) with linear gradients of 2-35\% acetonitrile 
in $0.1 \%$ formic acid, at a constant flow rate of $300 \mathrm{~nL} / \mathrm{min}$ for $220 \mathrm{~min}$. The instrument was operated in the positive-ion mode with the ESI spray voltage set at $1.8 \mathrm{kV}$. A full scan of MS spectra (300-1800 m/z) was acquired in the Q Exactive at a mass resolution of 70,000 at $200 \mathrm{~m} / \mathrm{z}$ with an automatic gain control target (AGC) of 3e6. Fifteen peptide ions showing the most intense signal from each scan were selected for a higher energy collision-induced dissociation (HCD)-MS/MS analysis (normalized collision energy 32) in the Q Exactive at a mass resolution of 35,000 and AGC value of 1e5. Maximal filling times were $100 \mathrm{~ms}$ in full scans and $120 \mathrm{~ms}$ (HCD) for the MS/MS scans. Ions with unassigned charge states and singly charged species were rejected. The dynamic exclusion was set to $50 \mathrm{~s}$ and a relative mass window of $10 \mathrm{ppm}$. Data were acquired using ThermoXcalibur 3.0.63. The phosphopeptides were analyzed under the same conditions as mentioned above, except that separation was done with linear gradients of $1-30 \%$ acetonitrile in $0.1 \%$ formic acid, and full scan MS ranging $300-2000 \mathrm{~m} / \mathrm{z}$ with NCE at 25.

Raw data were processed using SEQUEST with Proteome Discoverer (Version 2.1, Thermo Fisher Scientific) [24]. Data were searched against the mus musculus Universal Protein Resource sequence database (UniProt, August, 2013). The searches were performed with the following parameter settings: trypsin digestion with two missed cleavage allowed; fixed modification, carbamidomethyl of cysteine; variable modification, oxidation of methionine, TMT 10-plex (peptide labeled) for N terminus and Lys; MS tolerance, $5 \mathrm{ppm}$; MS/MS tolerance, $0.02 \mathrm{Da}$; false discovery rate (FDR) at peptide and protein levels, $<0.01$; and required peptide length, $\geq 6$ amino acids. Protein grouping was enabled, meaning if one protein was equal to or completely contained 
within the set of peptides of another protein, these two proteins were put into the same protein group. At least one unique peptide per protein group was required for identifying proteins. The relative protein abundance ratios (fold changes) between all blast and sham groups were calculated. As previously described, the changes in protein levels were considered significant if fold change was $>1.0$ (up-regulated) or $<1.0$ (down-regulated), and the $\mathrm{P}$ value is less than 0.05 in two independent experiments [20].

\subsubsection{Analysis of the mitochondrial respiratory chain}

Cortical tissues ( $\sim 20 \mathrm{mg}$ for each sample) were cut into small pieces and homogenized with a Dounce homogenizer in $1 \mathrm{~mL}$ of $20 \mathrm{mM}$ PBS containing protease and phosphatase inhibitors. The homogenate was diluted four folds with $20 \mathrm{mM}$ PBS and used to determine the enzymatic activities of Complex I (or NADH dehydrogenase), COX (or cytochrome C oxidase) and citrate synthase (CS) using an Infinite M200 plate reader (Tecan Group Ltd., Switzerland) as described previously [25]. The protein concentration of homogenate was determined using the DC (detergent compatible) protein assay (5000116, Bio-Rad Laboratories, CA). The maximal reaction speed (Vmax) normalized to the amount of protein was used to characterize the activity of each enzyme. For assay of Vmax of Complex I, diluted homogenate was first subjected to three freeze and thaw cycles. Next, $15 \mathrm{mM}$ Coenzyme Q1 was added into a cuvette containing $150 \mu \mathrm{M} \mathrm{NADH}$ and $40 \mu \mathrm{L}$ diluted homogenate. The decrease in NADH absorbance at $340 \mathrm{~nm}$ was monitored for 2 min for estimation of Vmax. To measure the Complex I induced NADH oxidation, $10 \mathrm{nM}$ of the Complex I inhibitor rotenone was added and the post-rotenone rate was subtracted from the pre-rotenone rate to yield the rotenone-sensitive NADH 
oxidation Vmax. For the COX Vmax assay, $25 \mu \mathrm{M}$ reduced cytochrome c was added into the cuvette containing $20 \mathrm{mM}$ PBS, $5 \mu \mathrm{l}$ diluted homogenate and $0.2 \mathrm{mg} / \mathrm{mL}$ dodecyl maltoside, to make the total volume of $1 \mathrm{~mL}$. The oxidation of the reduced cytochrome $\mathrm{c}$ was monitored for $2 \mathrm{~min}$ at $550 \mathrm{~nm}$ and the pseudo first-order rate constant was calculated as Vmax. For assay of citrate synthase Vmax activity, the substrates of CS, $100 \mu \mathrm{M}$ oxaloacetate and $50 \mu \mathrm{M}$ acetyl CoA, were added into the cuvette containing 100 $\mathrm{mM}$ Tris- $\mathrm{HCl}(\mathrm{pH} 8.0), 5 \mu 1$ homogenate and $100 \mu \mathrm{M}$ 5,5'-dithiobis-(2-nitrobenzoic acid) (DTNB) (1 mL total volume) and then the formation of 5-mercapto-2-nitrobenzoic acid was monitored for $2 \mathrm{~min}$ at $412 \mathrm{~nm}$, as previously described [25].

\subsubsection{Western blot analysis}

Protein samples were added to $4 \times$ SDS sample buffer with 1\% 2-mercaptoethanol and resolved on $12 \%, 1.5 \mathrm{~mm}$ SDS-PAGE gels as previously described [26]. Then proteins in SDS-PAGE gel were transferred to a nitrocellulose membrane. The membrane was first incubated in phosphate-buffered saline plus 0.1\% Tween-20 (PBST) (pH 7.4) containing $5 \%$ non-fat milk for $1 \mathrm{hr}$ at room temperature and then incubated with primary antibody, including COX IV (ab16056 Abcam, 1:2000); MnSOD (ab13534 Abcam, 1:3000); GPX4 (ab125066 Abcam, 1:2000); Drp1 (ab184247 Abcam, 1:1000); Fis1 (10956-1-AP Proteintech, 1:1000); OPA1 (cst80471 Cell Signaling Technology, 1:1000); Mitofusin 2 (cst9482 Cell Signaling Technology, 1:1000); VDAC1 (sc390996 Santa Cruz, 1:1000);

OXPHOS (containing a cocktail of complex I NDUFB8, complex II SDHB, complex III UQCRC2, complex IV MTCO1, and complex V ATP5A) (45-8099 ThermoFisher Scientific, 1:500); PINK1 (sc33796 Santa Cruz, 1:500); Parkin (cst4211 Cell Signaling 
Technology, 1:1000); ATG12 (cst4180 Cell Signaling Technology, 1:1000); LC3B (cst2775 Cell Signaling Technology, 1:1000); ULK1 (cst8054 Cell Signaling Technology, 1:1000); and Actin (A3854 Sigma-Aldrich, 1:15000) at $4{ }^{\circ} \mathrm{C}$ overnight. After washing with PBST three times, the membrane was incubated with corresponding secondary antibody (anti-mouse IgG, A0168 Sigma-Aldrich, 1:2000; anti-rabbit IgG, A0545 Sigma-Aldrich, 1:2000) in PBST for $1 \mathrm{hr}$ at room temperature. Immuno-reactive bands were detected using Amersham ECL Prime Western Blotting Detection Reagent and LAS-4000 Fujifilm imaging system.

\subsubsection{Bioinformatics and statistical analysis}

All quantitative proteomics data are normalized across all proteins within each 10-plex batch to eliminate the batch effect. Heatmap is generated by R package "pheatmap". Welch's test and t-distribution were performed with R function "t.test". PCA plots were generated with R package "prcomp" and the first three components were plotted with R package "rgl". Numbers of significantly change protein levels among different groups after blast (3 hrs, 24 hrs, 7 DPI and 30 DPI) vs sham control, were shown by Venn diagram with R package "VennDiagram" [27]. Pathway and gene ontology (GO) enrichment analysis was performed by R package "clusterProfiler". Proteins that have significant change in levels were enriched with Kyoto Encyclopedia of Genes and Genomes (KEGG) pathway database with function "enrichKEGG", and cellular component enrichment analysis was performed with function "enrichGO" [28]. The circularly composition analysis is performed with GO plot R packages as previously described [29]. All cutoffs for P value was set to 0.05 for enrichment analysis. 
Machine learning-based bioinformatic proteome analysis QIAGEN's Ingenuity Pathway Analysis (IPA, QIAGEN Redwood City, www.qiagen.com/ingenuity) was used to examine protein functions and molecular pathways associated with blast injury, as well as to predict protein-protein interaction networks, as previously described [30]. Z-score for IPA analysis was a statistical measure of the match between expected relationship direction and observed protein expression. Statistical analyses were performed with GraphPad Prism (version 6 for Windows; GraphPad Software). All data were expressed as means \pm SEM. Statistical comparisons were made by student's $t$ test for two groups and a one-way ANOVA test followed by Tukey test for multiple comparisons.

Differences were considered significant at $\mathrm{P}<0.05$ for all analyses, with * indicating $\mathrm{P}<0.05 ; * * \mathrm{P}<0.01 ; * * * \mathrm{P}<0.001 ; * * * * \mathrm{P}<0.0001$.

\subsection{RESULTS}

\subsubsection{Quantitative proteomic analysis of cortical global- and phospho-proteome profiles after primary LIB}

In order to provide a comprehensive understanding of the molecular events associated with dynamic changes occurring after primary LIB exposure, profiles of global- and phospho-proteomes in mouse cortex with or without blast injury were analyzed utilizing the TMT 10-plex tagging approach. For global proteome analysis, with criteria of FDR < $1 \%$ for both peptide and protein identification, we identified 2216 proteins in brain tissues after a $46.6 \mathrm{kPa}$ blast exposure. Among these, there were 170, 182, 108, and 173 differentially expressed proteins in $3 \mathrm{hrs}, 24 \mathrm{hrs}, 7$ DPI and 30 DPI groups as compared to sham control, respectively (Figure 5.1A-B). Using the phospho-proteome analysis by 
IMAC pull-down of phosphopeptides along with LC-MS/MS, we further identified 459 phosphorylated proteins. Specifically, there were $23,15,36$, and 41 differentially expressed phosphorylated proteins at $3 \mathrm{hrs,} 24 \mathrm{hrs}, 7 \mathrm{DPI}$, and $30 \mathrm{DPI}$ after the blast, respectively (Figure 5.1C-D). The Venn diagrams (Figure 5.1E) illustrated the changes in expression levels of individual proteins, especially some proteins in reoccurring or unique at various time points in the context of a primary LIB exposure. Expression levels of some proteins, such as NADH ubiquinone oxidoreductase subunits (NDUFA), inner membrane mitochondrial protein (IMMT), and voltage dependent anion channel 1 (VDAC1), changed at $3 \mathrm{hrs}$ after blast and remained altered up to 30 DPI.

To delineate relationships between sham and blast proteomes at different time points and to visualize the convergence and/or divergence among the proteomes, we performed PCA on the normalized expression values of the differentially expressed proteins. Results demonstrated consistency of these effects both in global- and phosphoproteome (Figure 5.1F). Among the PCA components, the blast groups were distinct from the sham control group. Nevertheless, various degrees of differences were found in individual blast groups, supporting the notion that even a single LIB exposure can drive significant impacts on protein expressions at different time points. 

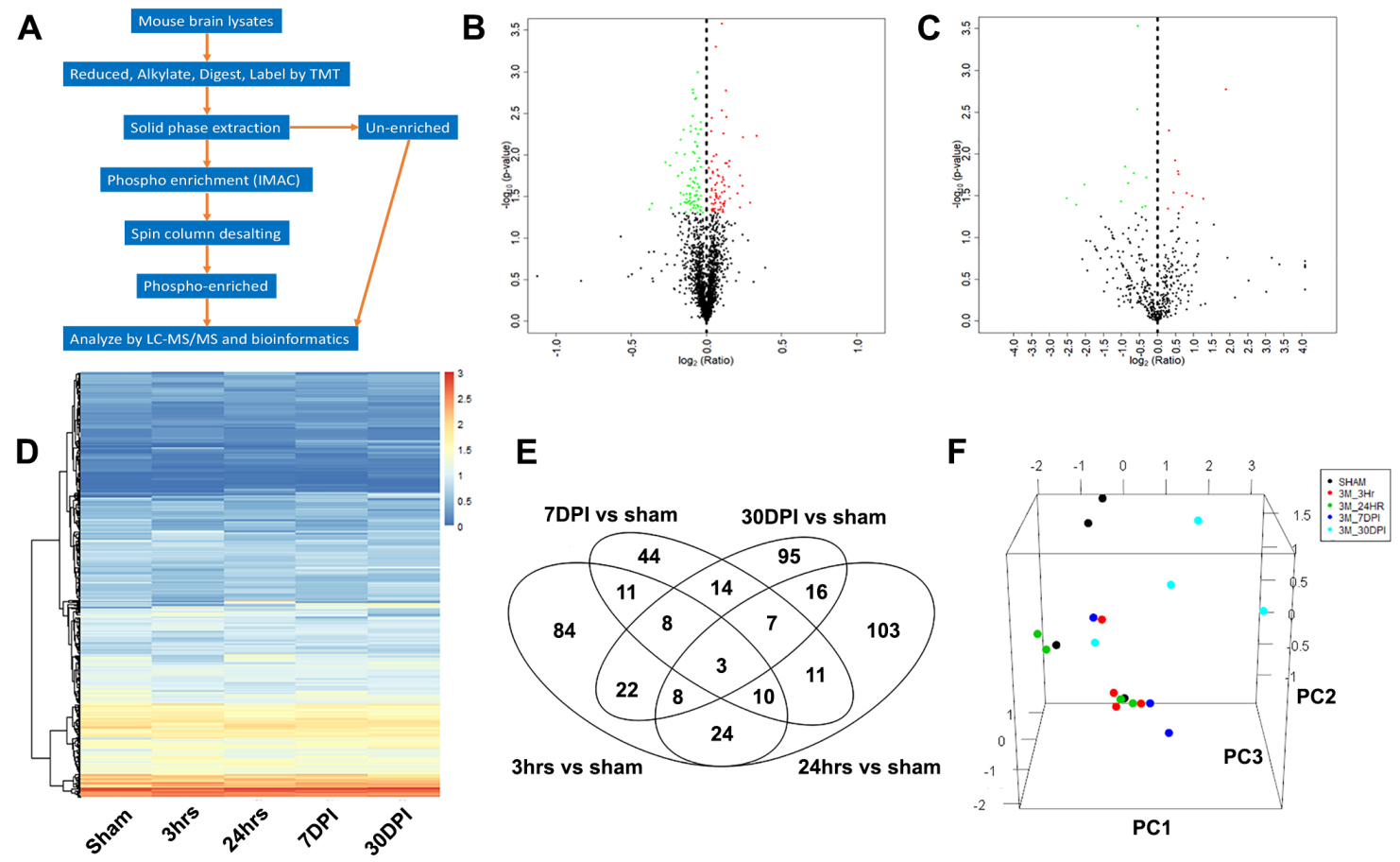

Figure 5.1 Proteomics analyses workflow and identification of differentially expressed global- and phospho- proteins after LIB injury.

(A) Workflow for quantitative profiling of mouse brain global- and phospho-proteome after blast exposure. (B-C) Low-intensity blast induced differentially expression of 170 global-proteins and 23 phospho-proteins at $3 \mathrm{hrs}$ time point, respectively. Volcano plots were generated by R statistical software. Dashed lines indicate the thresholds of $\log 2$ (ratio/fold change) and $-\log 10(\mathrm{P})$. The identified proteins are displayed as dots in red (up-regulation with significant changes), green (down-regulation with significant changes), or black color (no significant change). (D) Heatmap showing effects of LIB on phospho-protein expression over multiple time points. A large portion of blast induced up-/down-regulated phospho-proteins were dynamically changed along with the time after blast. For visualization, the calculated $-\log (\mathrm{P}$ value $)$ were shown in red as for upregulation and blue as for downregulation. The darkness of the color in each box 
reflects the absolute value of $-\log (\mathrm{P}$ value $)$. (E) Venn diagram showing the overlapping differentially expressed global-proteins among groups. The overlap portions indicate reoccurring proteins over time. (F) Principle component analysis showed that those differentially expressed global- proteins effectively separated blast at $3 \mathrm{hrs}$ (red), $24 \mathrm{hrs}$ (green), 7 DPI (dark blue), and 30 DPI (light blue) from sham control (black).

\subsubsection{GO annotation and canonical pathways associated with mitochondrial dysfunction after primary LIB}

In our previous studies, we observed persistent changes in brain mitochondria ultrastructure up to 30 DPI after LIB exposure [5]. In order to understand effects of primary LIB on dynamic molecular events, we mapped both global- and phosphoproteome identities to the GO cellular components, canonical signaling pathways, and regulatory networks potentially affected by blast at different time points, by applying KEGG pathways annotation and machine learning based IPA analysis [30]. In the cellular components of KEGG GO, the top ten represented categories include mitochondria (mitochondrial protein complex and mitochondrial inner membrane), myelin sheath, actin cytoskeleton, and synapse (presynapse, postsynapse, postsynaptic density, neuron to neuron synapse, and asymmetric synapse) (Figure 5.2A). Results shows other top ten shared or distinct cellular component details, comparing among blast groups at different time points in global- and phospho-proteome.

KEGG annotation and IPA pathway analysis showed that the top ten canonical pathways in blast affected global- and phospho-proteomes were categorized into mitochondrial dysfunction, oxidative stress (included oxidative phosphorylation, fatty 
acid degradation, glutathione metabolism, chemokine and mTOR signaling pathways, Nrf2 mediated oxidative stress response, synaptic dysfunction, axonal guidance signaling, regulation of cytoskeleton and gap junction, and neurodegeneration (related to Parkinson's diseases and Alzheimer's disease) (Figure 5.2B). The dynamics of involved signaling pathways, including oxidative stress and mitochondrial dysfunction, myelin sheath and axonal injury, and synaptic dysregulation pathways, changed along with different blast time points in both global- and phospho-proteomes (Figure 5.3A). From these signaling pathways, we observed various degrees of temporal changes in differentially expressed protein levels from $3 \mathrm{hrs}$ up to $30 \mathrm{DPI}$ related to mitochondrial dysfunction, axonal injury, and synaptic dysregulation (Table 5.1-5.3). Particularly, these changes included multiple proteins associated with oxidative stress and mitochondrial dysfunction pathways. As shown in Table 5.1 these included NDUFA, ubiquinolcytochrome $\mathrm{c}$ reductase core protein 2 (UQCRC2), ATP synthase (ATP5PB and ATP5PD) (oxidative phosphorylation), mitochondrial isocitrate dehydrogenase (IDH2) (peroxisome), and mitofusin 2 (MFN2) (fission-fusion and mitophagy). 

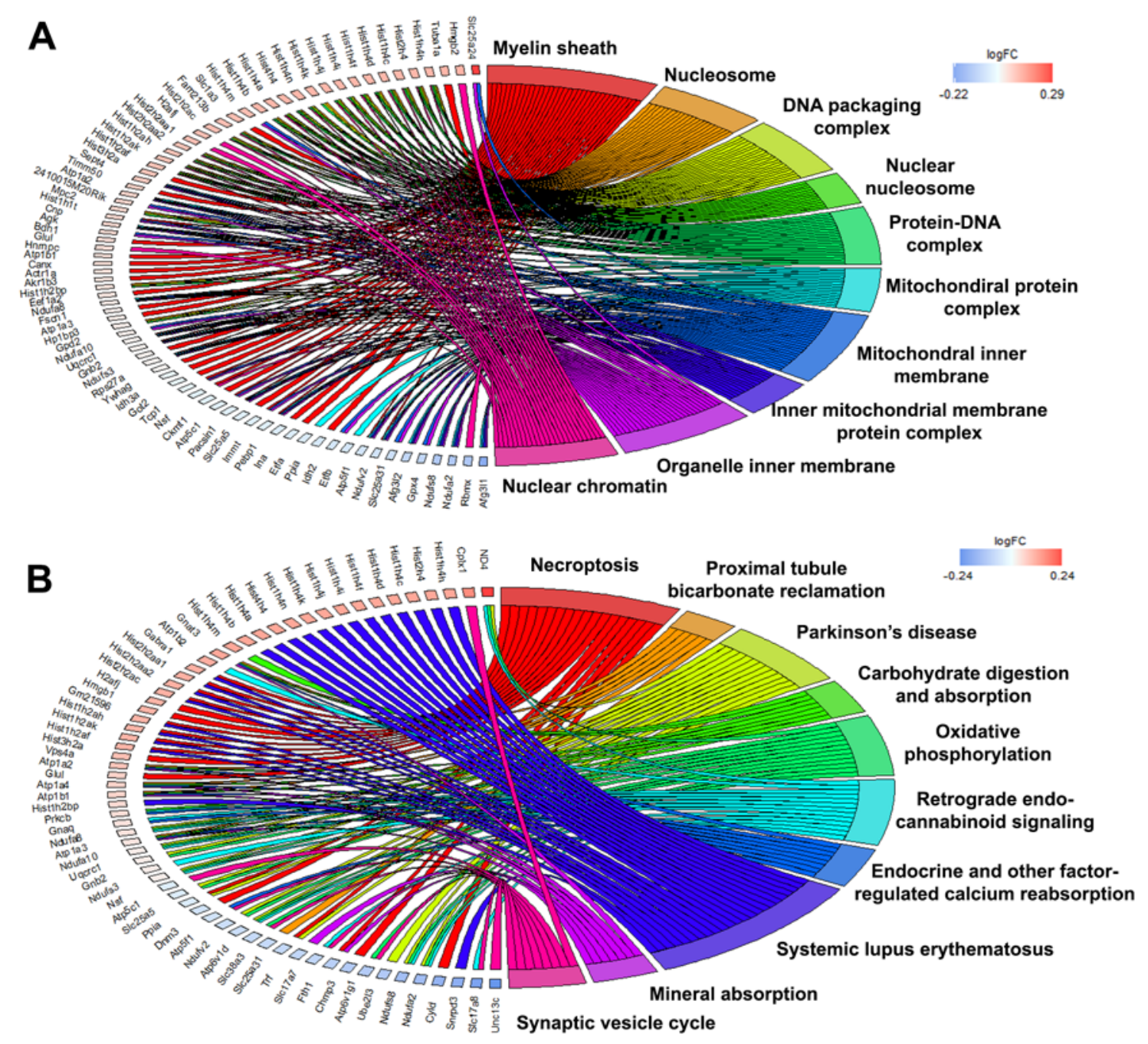

Figure 5.2 Cellular components and signaling pathways enriched in global proteome

\section{at 3 hrs after LIB injury.}

(A) Global proteins enriched in the top shared cellular components. (B) Global proteins enriched in the top shared functional pathways. Each cluster of cellular components and canonical pathways in the plot is assigned a unique color. Each connection between a protein and the cellular component or canonical pathway represents the fold change. 
Table 5.1 Canonical pathways annotation from global-proteome associated with oxidative stress and mitochondrial dysfunction after LIB injury.

\begin{tabular}{|c|c|c|c|c|c|}
\hline \multicolumn{2}{|c|}{ Canonical pathway } & \multicolumn{4}{|c|}{ Differentially expressed proteins } \\
\hline ID & Name & $3 \mathrm{hrs}$ & $24 \mathrm{hrs}$ & 7DPI & 30DPI \\
\hline \multirow[t]{2}{*}{ mmu00190 } & $\begin{array}{l}\text { Oxidative } \\
\text { phosphorylation }\end{array}$ & $\begin{array}{l}\text { Ndufa2, } \\
\text { Ndufs8 }\end{array}$ & $\begin{array}{l}\text { Uqcrc1, } \\
\text { Ndufs3, } \\
\text { Ndufa2, } \\
\text { Uqcrc2, } \\
\text { Atp5h, } \\
\text { Atp5f1, } \\
\text { Ppa1 }\end{array}$ & Atp5f1 & $\begin{array}{l}\text { Atp5c1, } \\
\text { Ndufa2 }\end{array}$ \\
\hline & & $2.90 \mathrm{E}-05$ & $5.81 \mathrm{E}-04$ & $4.93 \mathrm{E}-05$ & $3.52 \mathrm{E}-03$ \\
\hline \multirow[t]{2}{*}{ mmu04137 } & Mitophagy & NA & Ubb & $\begin{array}{l}\text { Mfn2, } \\
\text { Rras2 }\end{array}$ & NA \\
\hline & & $1.13 \mathrm{E}-01$ & $8.50 \mathrm{E}-01$ & $8.16 \mathrm{E}-01$ & $4.02 \mathrm{E}-01$ \\
\hline \multirow[t]{2}{*}{ mmu04140 } & Autophagy & NA & $\begin{array}{l}\text { Lamp1, } \\
\text { Rragb, } \\
\text { Prkcq, } \\
\text { Mapk3, } \\
\text { Camkk2 }\end{array}$ & Rras2 & Rragb \\
\hline & & $6.90 \mathrm{E}-02$ & $3.01 \mathrm{E}-02$ & $8.41 \mathrm{E}-01$ & $5.05 \mathrm{E}-01$ \\
\hline $\mathrm{mmu} 04142$ & Lysosome & NA & $\begin{array}{l}\text { Lamp1, } \\
\text { Ap3b2 }\end{array}$ & Ap3b2 & Cltb \\
\hline
\end{tabular}




\begin{tabular}{|c|c|c|c|c|c|}
\hline & & 4.89E-01 & $5.01 \mathrm{E}-01$ & $4.14 \mathrm{E}-01$ & $5.09 \mathrm{E}-01$ \\
\hline \multirow[t]{2}{*}{ mmu04145 } & \multirow[t]{2}{*}{ Phagosome } & $\begin{array}{l}\text { Tuba1a, } \\
\text { Dync1h1, } \\
\text { Tuba3a }\end{array}$ & $\begin{array}{l}\text { Lamp1, } \\
\text { Tuba1b, } \\
\text { Rab5a, } \\
\text { Rab5c }\end{array}$ & $\begin{array}{l}\text { Dync1h1, } \\
\text { Rab5c }\end{array}$ & NA \\
\hline & & $6.89 \mathrm{E}-03$ & $1.98 \mathrm{E}-02$ & $2.52 \mathrm{E}-02$ & $4.30 \mathrm{E}-05$ \\
\hline \multirow[t]{2}{*}{ mmu04146 } & \multirow[t]{2}{*}{ Peroxisome } & Eci2, Idh2 & Eci2 & Eci2 & Idh2 \\
\hline & & $1.52 \mathrm{E}-01$ & $1.58 \mathrm{E}-01$ & $2.88 \mathrm{E}-01$ & $2.88 \mathrm{E}-01$ \\
\hline \multirow[t]{2}{*}{$\mathrm{mmu} 04150$} & \multirow[t]{2}{*}{$\begin{array}{l}\text { mTOR signaling } \\
\text { pathway }\end{array}$} & NA & $\begin{array}{l}\text { Rragb, } \\
\text { Mapk3 }\end{array}$ & NA & Rragb \\
\hline & & $1.47 \mathrm{E}-01$ & $7.60 \mathrm{E}-02$ & $3.60 \mathrm{E}-01$ & $2.27 \mathrm{E}-01$ \\
\hline \multirow[t]{2}{*}{ mmu04210 } & \multirow[t]{2}{*}{ Apoptosis } & $\begin{array}{l}\text { Tubala, } \\
\text { Tuba3a }\end{array}$ & $\begin{array}{l}\text { Tubalb, } \\
\text { Mapk3 }\end{array}$ & Spta1 & NA \\
\hline & & $6.06 \mathrm{E}-02$ & $4.25 \mathrm{E}-01$ & $3.33 \mathrm{E}-01$ & $6.13 \mathrm{E}-01$ \\
\hline
\end{tabular}

(Note: P values for each specific canonical pathways at different time points are presented directly under each pathway at different time points. NA, no differentially expressed protein is identified.)

Table 5.2 Canonical pathways annotation from global-proteome associated with myelin sheath and axonal injury after LIB injury.

\begin{tabular}{|l|l|l|l|l|l|}
\hline \multicolumn{4}{|l|}{ Canonical pathway } & \multicolumn{4}{l|}{ Differentially expressed proteins } \\
\hline ID & Name & $3 \mathrm{hrs}$ & $24 \mathrm{hrs}$ & 7 DPI & $30 \mathrm{DPI}$ \\
\hline
\end{tabular}




\begin{tabular}{|c|c|c|c|c|c|}
\hline \multirow[t]{2}{*}{$\begin{array}{l}\mathrm{mmu} 0436 \\
0\end{array}$} & \multirow[t]{2}{*}{$\begin{array}{l}\text { Axon } \\
\text { guidance }\end{array}$} & Cdk5 & $\begin{array}{l}\text { Cdk5, } \\
\text { Gnai3, } \\
\text { Mapk3, } \\
\text { Ntng1 }\end{array}$ & NA & NA \\
\hline & & $3.55 \mathrm{E}-01$ & $3.68 \mathrm{E}-01$ & $9.37 \mathrm{E}-01$ & $9.75 \mathrm{E}-01$ \\
\hline \multirow[t]{2}{*}{$\begin{array}{l}\mathrm{mmu} 0481 \\
0\end{array}$} & \multirow[t]{2}{*}{$\begin{array}{l}\text { Regulation } \\
\text { of actin } \\
\text { cytoskeleto } \\
\mathrm{n}\end{array}$} & Nckap1 & $\begin{array}{l}\text { Myh9, Wasl, } \\
\text { Nckap1, } \\
\text { Mapk3, } \\
\text { Wasf2 }\end{array}$ & Rras2 & Nckap1 \\
\hline & & $2.86 \mathrm{E}-02$ & $2.03 \mathrm{E}-05$ & 5.94E-01 & $4.25 \mathrm{E}-01$ \\
\hline \multirow[t]{2}{*}{$\begin{array}{l}\mathrm{mmu} 0407 \\
1\end{array}$} & \multirow{2}{*}{$\begin{array}{l}\text { Sphingolipi } \\
\text { d signaling } \\
\text { pathway }\end{array}$} & NA & $\begin{array}{l}\text { Gnai3, } \\
\text { Mapk3 }\end{array}$ & Gnaq & Gnaq \\
\hline & & $2.86 \mathrm{E}-02$ & $2.03 \mathrm{E}-05$ & $5.94 \mathrm{E}-01$ & $4.25 \mathrm{E}-01$ \\
\hline \multirow[t]{2}{*}{$\begin{array}{l}\mathrm{mmu} 0451 \\
4\end{array}$} & \multirow{2}{*}{$\begin{array}{l}\text { Cell } \\
\text { adhesion } \\
\text { molecules } \\
\text { (CAMs) }\end{array}$} & NA & $\begin{array}{l}\text { Nrxn3, } \\
\text { Negr1, } \\
\text { Ntng1 }\end{array}$ & NA & NA \\
\hline & & $6.38 \mathrm{E}-01$ & $6.49 \mathrm{E}-01$ & $1.00 \mathrm{E}+00$ & $8.41 \mathrm{E}-01$ \\
\hline
\end{tabular}

Table 5.3 Canonical pathways annotation from global-proteome associated with synaptic dysregulation after LIB injury.

\begin{tabular}{|l|l|l|l|l|l|}
\hline \multicolumn{2}{|l|}{ Canonical pathway } & \multicolumn{4}{l|}{ Differentially expressed proteins } \\
\hline ID & Name & $3 \mathrm{hrs}$ & $24 \mathrm{hrs}$ & 7 DPI & $30 \mathrm{DPI}$ \\
\hline & & NA & NA & Vamp2 & Cltb \\
\hline
\end{tabular}




\begin{tabular}{|c|c|c|c|c|c|}
\hline $\begin{array}{l}\mathrm{mmu} 0472 \\
1\end{array}$ & $\begin{array}{l}\text { Synaptic } \\
\text { vesicle cycle }\end{array}$ & $6.24 \mathrm{E}-03$ & $1.44 \mathrm{E}-03$ & $1.24 \mathrm{E}-04$ & $3.18 \mathrm{E}-05$ \\
\hline \multirow[t]{2}{*}{$\begin{array}{l}\mathrm{mmu} 0472 \\
7\end{array}$} & \multirow[t]{2}{*}{$\begin{array}{l}\text { GABAergic } \\
\text { synapse }\end{array}$} & Gnb2, Glul & $\begin{array}{l}\text { Gnb2, } \\
\text { Gnai3, Glul, } \\
\text { Slc38a3 }\end{array}$ & Gnb2 & $\begin{array}{l}\text { Glul, } \\
\text { Slc38a3 }\end{array}$ \\
\hline & & $1.97 \mathrm{E}-03$ & $1.88 \mathrm{E}-05$ & $1.71 \mathrm{E}-02$ & $2.95 \mathrm{E}-04$ \\
\hline \multirow[t]{2}{*}{$\begin{array}{l}\mathrm{mmu} 0472 \\
4\end{array}$} & \multirow[t]{2}{*}{$\begin{array}{l}\text { Glutamatergi } \\
\text { c synapse }\end{array}$} & $\begin{array}{l}\text { Gnb2, Glul, } \\
\text { Shank3 }\end{array}$ & $\begin{array}{l}\text { Gnb2, } \\
\text { Gnai3, Glul, } \\
\text { Mapk3, } \\
\text { Slc38a3, } \\
\text { Shank3 }\end{array}$ & $\begin{array}{l}\text { Gnb2, Gnaq, } \\
\text { Grk2, } \\
\text { Shank3 }\end{array}$ & $\begin{array}{l}\text { Gnaq, Glul, } \\
\text { Slc38a3, } \\
\text { Grk2 }\end{array}$ \\
\hline & & $1.78 \mathrm{E}-05$ & $9.48 \mathrm{E}-05$ & $1.48 \mathrm{E}-04$ & $9.88 \mathrm{E}-03$ \\
\hline \multirow[t]{2}{*}{$\begin{array}{l}\mathrm{mmu} 0472 \\
0\end{array}$} & \multirow[t]{2}{*}{$\begin{array}{l}\text { Long-term } \\
\text { potentiation }\end{array}$} & $\begin{array}{l}\text { Gnb2, Glul, } \\
\text { Shank3 }\end{array}$ & $\begin{array}{l}\text { Gnb2, } \\
\text { Gnai3, Glul, } \\
\text { Mapk3, } \\
\text { Slc38a3, } \\
\text { Shank3 }\end{array}$ & $\begin{array}{l}\text { Gnb2, Gnaq, } \\
\text { Grk2, } \\
\text { Shank3 }\end{array}$ & $\begin{array}{l}\text { Gnaq, Glul, } \\
\text { Slc38a3, } \\
\text { Grk2 }\end{array}$ \\
\hline & & $4.46 \mathrm{E}-02$ & $1.39 \mathrm{E}-02$ & $2.56 \mathrm{E}-01$ & $1.76 \mathrm{E}-01$ \\
\hline \multirow[t]{2}{*}{$\begin{array}{l}\mathrm{mmu} 0473 \\
0\end{array}$} & \multirow[t]{2}{*}{$\begin{array}{l}\text { Long-term } \\
\text { depression }\end{array}$} & NA & $\begin{array}{l}\text { Gnai3, } \\
\text { Mapk3 }\end{array}$ & Gnaq & Gnaq \\
\hline & & $1.08 \mathrm{E}-02$ & $1.16 \mathrm{E}-02$ & $2.38 \mathrm{E}-01$ & $7.55 \mathrm{E}-01$ \\
\hline
\end{tabular}


Based upon the analysis of canonical pathways, we further characterized the blast effects relating to brain injuries at the molecular level by IPA to predict the toxic annotation. Bioinformatic analysis indicated that this level of LIB exposure caused mitochondrial dysfunction (associated with decreased depolarization and transmembrane potential of mitochondria and mitochondrial membrane, biogenesis of mitochondria) and damage due to oxidative stress (involved with fatty acid metabolism and Nrf2 mediated oxidative stress response). These findings were reflected by up- and down-regulation of specific mitochondrial proteins, including glutathione peroxidase 4 (GPX4), NDUFA, ATP5PB and ATP5PD, UQCRC2, IMMT, and protein kinase C (PRKC); and by upregulation of phosphorylated proteins, such as VDAC1, stathmin 1 (STMN1), and ATPase sarcoplasmic/endoplasmic reticulum calcium transporting 2 (ATP2A2) (Figure 5.3B). The differential expression of multiple key mitochondrial proteins related to oxidative phosphorylation, respiration and electron transport chain (ETC) function demonstrates that LIB exposure causes bioenergetic failure. 

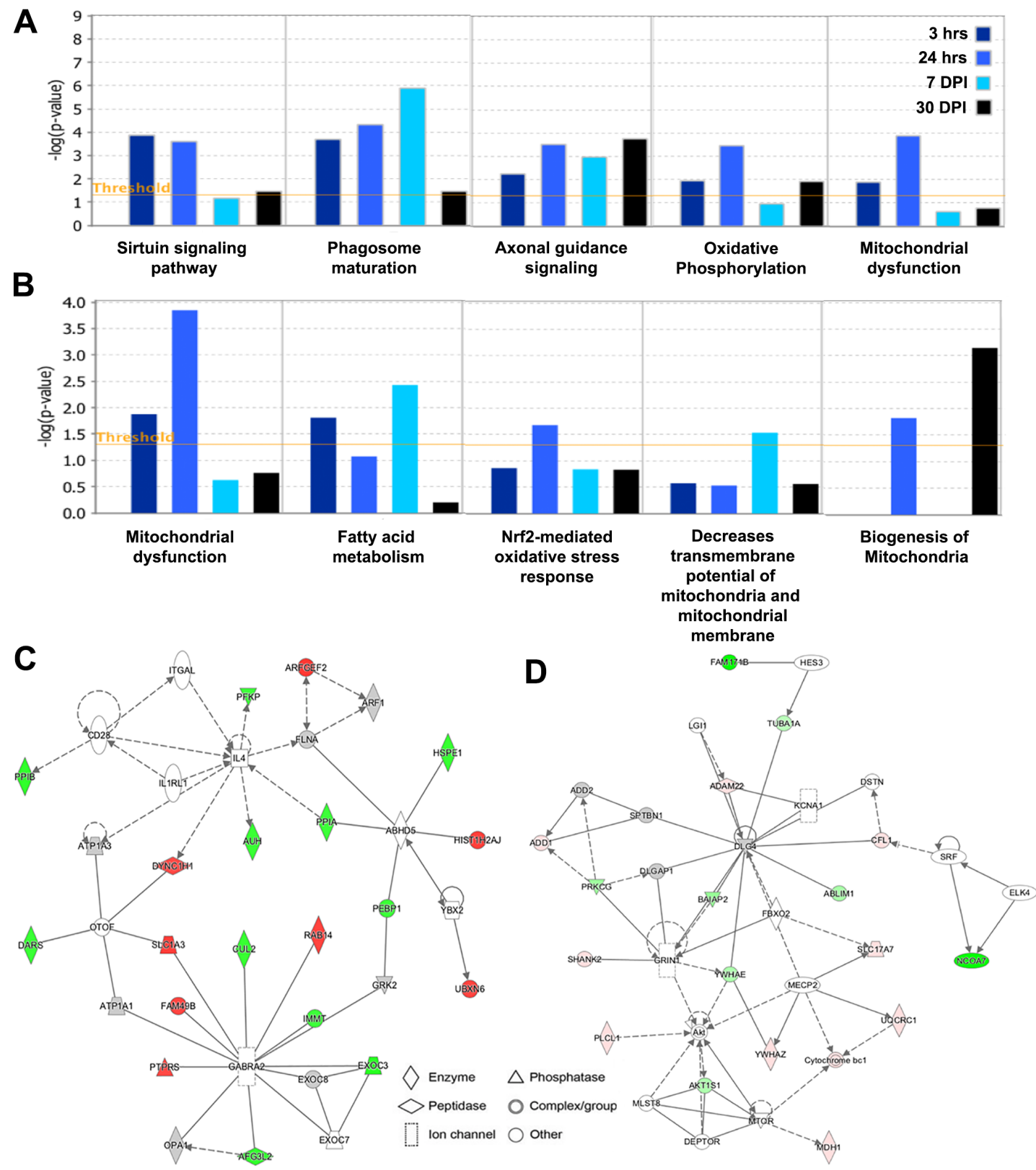

Figure 5.3 LIB effects at different time on alterations of canonical pathways, toxic annotation, and disease/function networks.

(A) Top five canonical pathways predicted by an IPA analysis of the differentially expressed global- and phospho-proteins affected by the low-intensity blast exposure at different time points (color coded). (B) Top five toxic lists predicted by IPA. The canonical pathways and toxic list annotations were ranked according to the -log (P value). 
A ratio (height) indicates the number of proteins that were differentially expressed in each pathway or list over the total numbers of proteins in that specific pathway. (C) The top disease/function network in global-proteome is associated with cellular development, cellular growth and proliferation, cellular movement, and cellular assembly and organization corresponding to the low-intensity blast. (D) The top disease/function network in phospho-proteome is associated with neurological disease, organismal injury and abnormalities, cell-to-cell signaling and interaction, and cell morphology corresponding to the low-intensity blast. The identified genes involved in the networks were displayed in red (up-regulation) and green (down-regulation) color. The color intensity indicates the degree of regulation. Solid lines in the network imply direct interactions between genes, and dashed lines indicate indirect interactions. Geometric shapes represent different general functional families of gene regulation (diamond for enzyme, oval for transcription regulator, trapezoid for transporter, inverted triangle for kinase, double circle for complex/group, and circle for others).

\subsubsection{Annotation of disease and functional networks regulated after primary LIB}

To further investigate functional links among the differentially expressed proteins as well as related biological processes in context of the LIB exposure, IPA was used to map the global- and phospho-proteome identities to reveal signaling pathways and regulatory networks. The top disease / function networks induced by the blast injury in globalproteome were involved with cellular development, cellular growth and proliferation, cellular movement, and cellular assembly and organization (Figure 5.3C). This network was associated with downregulation of IMMT, mitochondrial dynamin-related GTPase 
(OPA1), cullin-2 (CUL2) (core component of E3 ubiquitin-protein ligase complexes), synaptosomal-associated protein 25 (SNAP25); and upregulation of MAP kinaseactivated protein kinase 3 (MAPK3), peroxiredoxin-6 (PRDX6) (redox activity) at various time points after blast. By contrast, the top networks, associated with blast signaled down regulation of leucine-rich repeat-containing protein 7 (LRRC7) (required

for normal synaptic spine architecture and function), tropomodulin-2 (TMOD2) (regulating membrane skeleton), and ubiquitin-like modifier-activating enzyme 1 (UBA1); upregulated STMN1 (destabilizing microtubules) and VDAC1, related to phospho-proteome include neurological disease, organismal injury and abnormalities, cell-to-cell signaling and interaction, and cell morphology (Figure 5.3D). Importantly, in consistent with the canonical pathway findings, the disease / functional network data further confirms mitochondrial dysfunction after LIB-induced brain injury.

\subsubsection{Primary LIB induced oxidative stress associated with mitochondrial}

\section{dysfunction}

Driven by quantitative proteomics and machine learning based bioinformatics analysis, we investigated specific mitochondrial related molecular and biochemical events over time in mice exposed to primary LIB. MnSOD, a well-documented mitochondrial oxidative stress marker, was progressively and significantly elevated at $24 \mathrm{hrs}$ and $7 \mathrm{DPI}$, with an increased trend at 30 DPI after blast exposure (Figure 5.4A-B). Additionally, we observed that GPX4 level was significantly increased at $24 \mathrm{hrs}$ after LIB injury (Figure 5.4C). GPX4 expression returned to sham control level at 30 DPI. These results suggest 
that primary LIB blast exposure increases levels of oxidative stress associated with mitochondrial dysfunction.

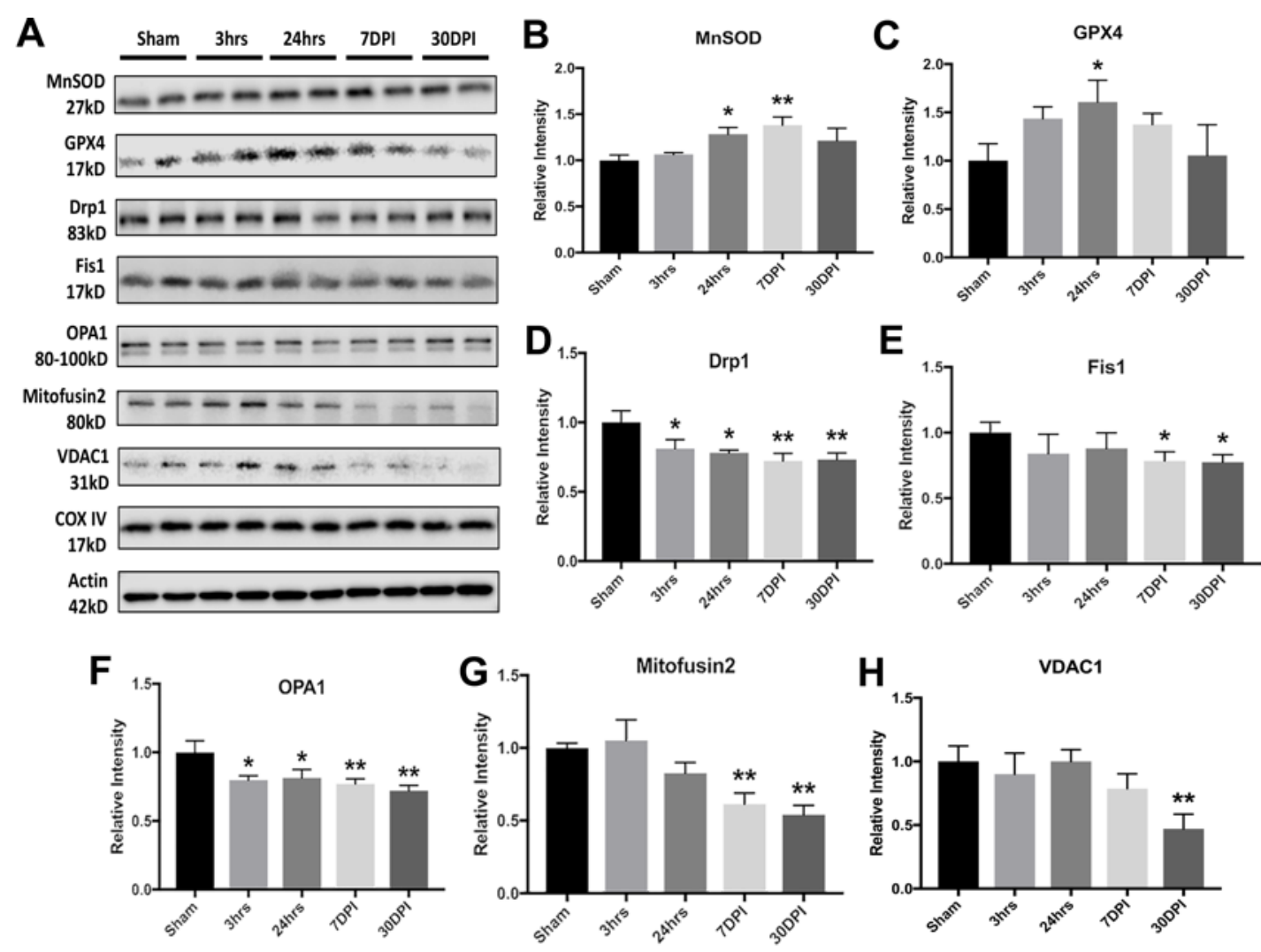

Figure 5.4 LIB induced mitochondrial dysfunctions associated with oxidative stress and fission-fusion dynamics.

(A) Western blot and analysis of mitochondrial markers associated with oxidative stress and fission-fusion dynamics after low-intensity blast. (B) Significant increase of MnSOD levels was found at $24 \mathrm{hrs}(\mathrm{P}<0.05)$ and $7 \mathrm{DPI}(\mathrm{P}<0.01)$ after blast. (C) GPX4 level was significantly elevated at $24 \mathrm{hrs}(\mathrm{P}<0.05)$ after blast. (D) Drp1 expression was significantly decreased at all time points after blast as compared to sham $(\mathrm{P}<0.05)$. (E) Fis 1 expression was significantly decreased at 7 and 30 DPI (P<0.05). (F) OPA1 level was significantly decreased at all time points after blast $(\mathrm{P}<0.05)$. (G) Significant decrease of Mitofusin2 levels was observed at 7 and 30 DPI $(\mathrm{P}<0.01)$. (H) Significant 
decrease of VDAC1 was identified at $30 \mathrm{DPI}(\mathrm{P}<0.01)$. $\mathrm{N}=4$ for each individual group for comparison. All data are expressed as mean \pm SEM and were analyzed by one-way ANOVA test followed by Tukey test for multiple comparisons. Differences were considered significant at $\mathrm{P}<0.05$ for all analyses, with * indicating $\mathrm{P}<0.05$; $* * \mathrm{P}<0.01$.

\subsubsection{Primary LIB altered mitochondrial dynamics, enzymatic activity on bioenergetics and compromised mitophagy}

Next, we investigated molecular changes after LIB associated with mitochondrial fissionfusion dynamics, mitophagy, and autophagy. In this experiment, corroborated with proteomics identification, changes in fission and fusion activities were evidenced by significantly decreased Drp1 (mitochondrial fission), Fis1 (mitochondrial fission and recruitment / association of Drp1), OPA1 (inner-membrane fusion), and mitofusin 2 (outer-membrane fusion) levels mainly at 7 and 30 DPI (Figure 5.4D-G). Levels of VDAC1, associated with mitochondrial volume regulation, was also reduced at 30 DPI (Figure 5.4H).

We further identified mitochondrial bioenergetic failure after LIB exposure by assessing mitochondrial oxidative phosphorylation (OXPHOS) essential for ATP production, and measuring mitochondrial respiration chain enzymatic activity. We found significantly decreased levels of the complex V ATP5A and complex III UQCRC2 subunits at $3 \mathrm{hrs}$ and $30 \mathrm{DPI}$ after blast injury (Figure 5.5A-C). The results validated the proteomics identifications. The Complex IV MTCO1 subunit was also reduced at 3 and $24 \mathrm{hrs}$ after blast (Figure 5.5D). Additionally, we observed that complex II SDHB subunit expression significantly decreased at $24 \mathrm{hrs}$ (Figure 5.5E). Although no significant 
change was identified in the complex I NDUFB8 subunit protein (Figure 5.5F), the blast at $24 \mathrm{hrs}$ post injury led to a significant increase of complex I activity (Figure 5.5G). Activity of COX and CS (a matrix-soluble enzyme whose levels tend to reflect mitochondrial mass) showed similar trends in mice exposed to blast as compared to sham (Figure 5.5H-I). Decreased levels of multiple OXPHOS subunit proteins and increased complex I Vmax activity suggest LIB alters mitochondria, which secondarily compensate for either LIB-induced dysfunction or for increased energy needs.

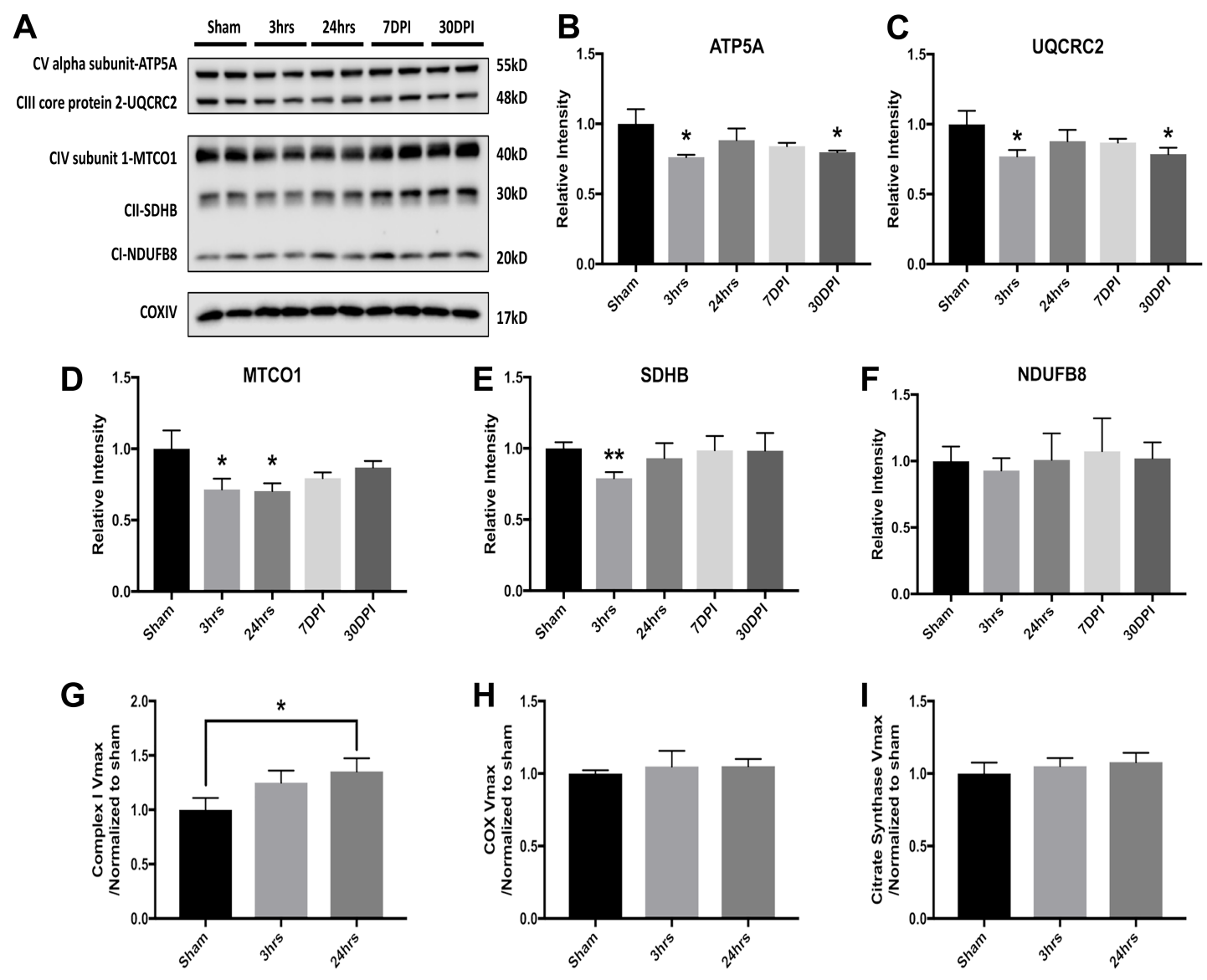

Figure 5.5 LIB induced mitochondrial oxidative phosphorylation and bioenergetic failure.

(A) Western blot and analysis of mitochondrial markers associated with oxidative phosphorylation after low-intensity blast. (B) Significant decreases of complex V ATP5A 
levels were found at 3 hrs $(\mathrm{P}<0.05)$ and 30 DPI $(\mathrm{P}<0.05)$ after blast. (C) Complex III UQCRC2 levels were significantly reduced at $3 \mathrm{hrs}(\mathrm{P}<0.05)$ and $30 \mathrm{DPI}(\mathrm{P}<0.05)$ after blast. (D) Complex IV MTCO1 expressions were significantly decreased at $3 \mathrm{hrs}$ and 24 hrs after blast as compared to sham $(\mathrm{P}<0.05)$. (E) Complex II SDHB expression was significantly decreased at $24 \mathrm{hrs}(\mathrm{P}<0.01)$. (F) No significant change was identified for complex I NDUFB8 protein expression level after blast. (G) However, significant elevated complex I enzyme activity was found at 24 hrs after blast $(\mathrm{P}<0.05)$. (H-I) COX and CS activities assessed after blast injury (no significant differences among comparisons). $\mathrm{N}=4$ for each individual group for comparison. All data are expressed as mean \pm SEM and were analyzed by one-way ANOVA test followed by Tukey test for multiple comparisons. Differences were considered significant at $\mathrm{P}<0.05$ for all analyses, with * indicating $\mathrm{P}<0.05 ; * * \mathrm{P}<0.01$.

In order to further examine changes in markers related to mitophagy and autophagy, results showed significantly decreased levels of Parkin, and with a trend for PINK1, at 7 and 30 DPI (Figure 5.6A-C). These changes suggest efforts to eliminate damaged mitochondria. Significant decreased levels of ATG12 (involved in the processes of autophagic initiation and elongation to engulf cytoplasmic components), with lower trends for ULK1 / ATG1 and LC3B, from 24 hrs to $30 \mathrm{DPI}$, suggested impaired autophagy by reducing the maturation of functional autophagosome and subsequent attenuation of autophagic flux after blast injury (Figure 5.6D). Taken together, these results are in agreement with proteomics and bioinformatics data demonstrating primary LIB-induced mitochondrial dysfunction, particularly involved with increased oxidative 
stress, impaired fission-fusion dynamics, diminished mitophagy, and acute compensatory respiration activity.
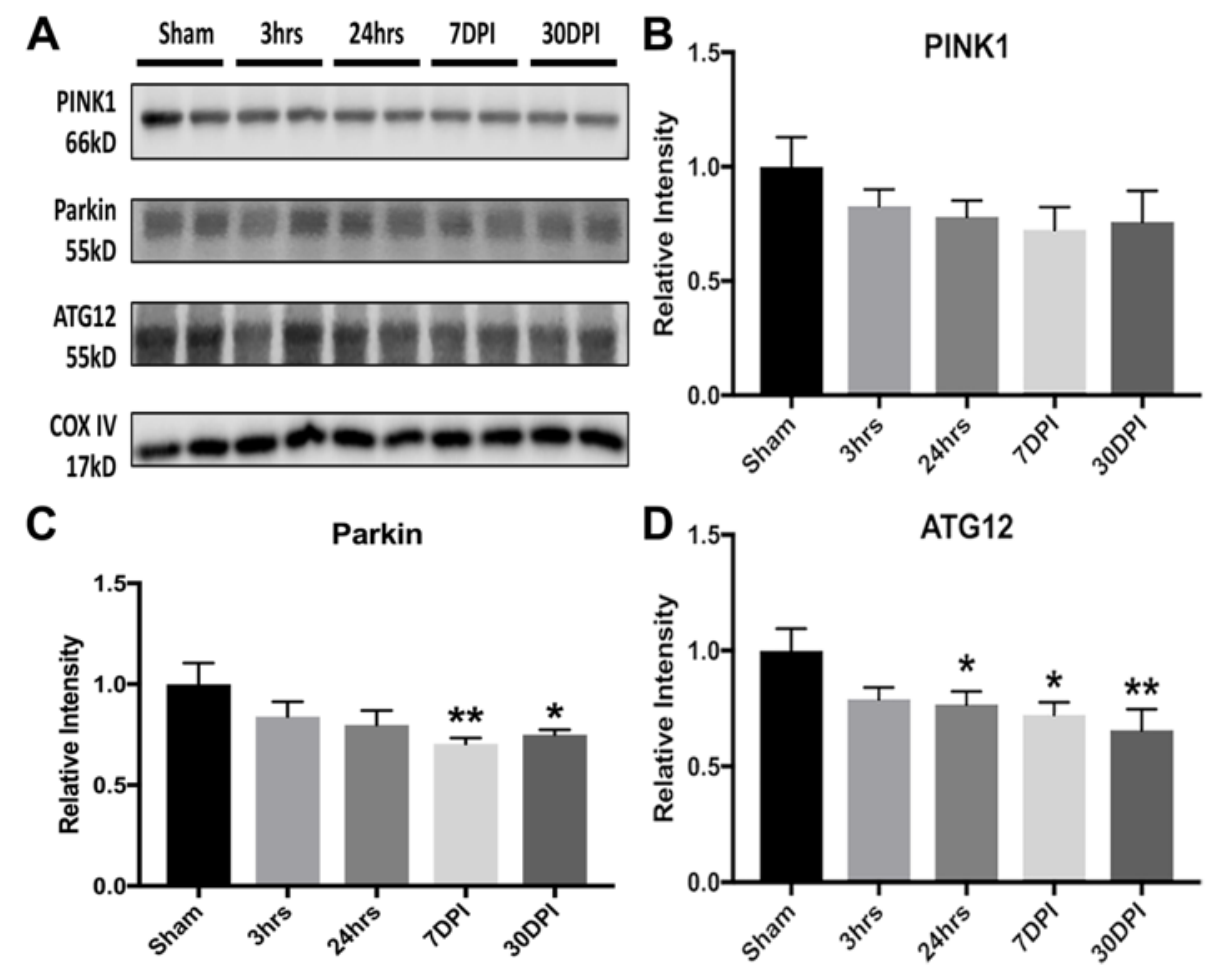

Figure 5.6 LIB induced mitochondrial dysfunctions associated with mitophagy and autophagy.

(A) Western blot and analysis of mitochondrial markers associated with mitophagy and autophagy after low-intensity blast. (B) PINK1 expression was trended lower after blast as compared to sham. (C) Parkin expression was significantly decreased at $7(\mathrm{P}<0.01)$ and 30 DPI $(\mathrm{P}<0.05)$. (D) Significant decrease of ATG12 levels, with a trend for LC3B, was found at 24hrs $(\mathrm{P}<0.05), 7(\mathrm{P}<0.05)$ and $30 \mathrm{DPI}(\mathrm{P}<0.01) . \mathrm{N}=4$ for each individual group for comparison. All data are expressed as mean \pm SEM and were analyzed by oneway ANOVA test followed by Tukey test for multiple comparisons. Differences were considered significant at $\mathrm{P}<0.05$ for all analyses, with * indicating $\mathrm{P}<0.05$; ** $\mathrm{P}<0.01$. 


\subsection{DISCUSSION}

Explosive blast induced mTBI, a "signature wound" of the recent military conflicts remains a serious concern. While experimental animal studies of high intensity blast exposures with over 100-kPa peak overpressure have provided insights into neurological damage due to moderate or severe TBI, the pathobiology and underlying mechanisms of mild brain injury by primary blast wave require further investigation. In this context, we have characterized details of mitochondrial dysfunction involved with increased oxidative stress, impaired fission-fusion dynamics, diminished mitophagy, decreased oxidative phosphorylation, and acute compensated respiration activity by using quantitative proteomic analyses of the effects of primary LIB up to 30 days after exposure, as depicted by a scheme in Figure 5.7. We used this military-relevant mouse model of LIB brain injury with a magnitude of $46.6 \mathrm{kPa}$ peak overpressure and $8.7 \mathrm{PSI} \times$ ms maximum impulse [5,6]. The temporal profiles of global- and phospho- proteome changes at acute (3 and $24 \mathrm{hrs}$ ), subacute (7 DPI), and longer term (30 DPI) time points after LIB exposure exhibited striking sequential changes some of which persisted up to 30 DPI. 


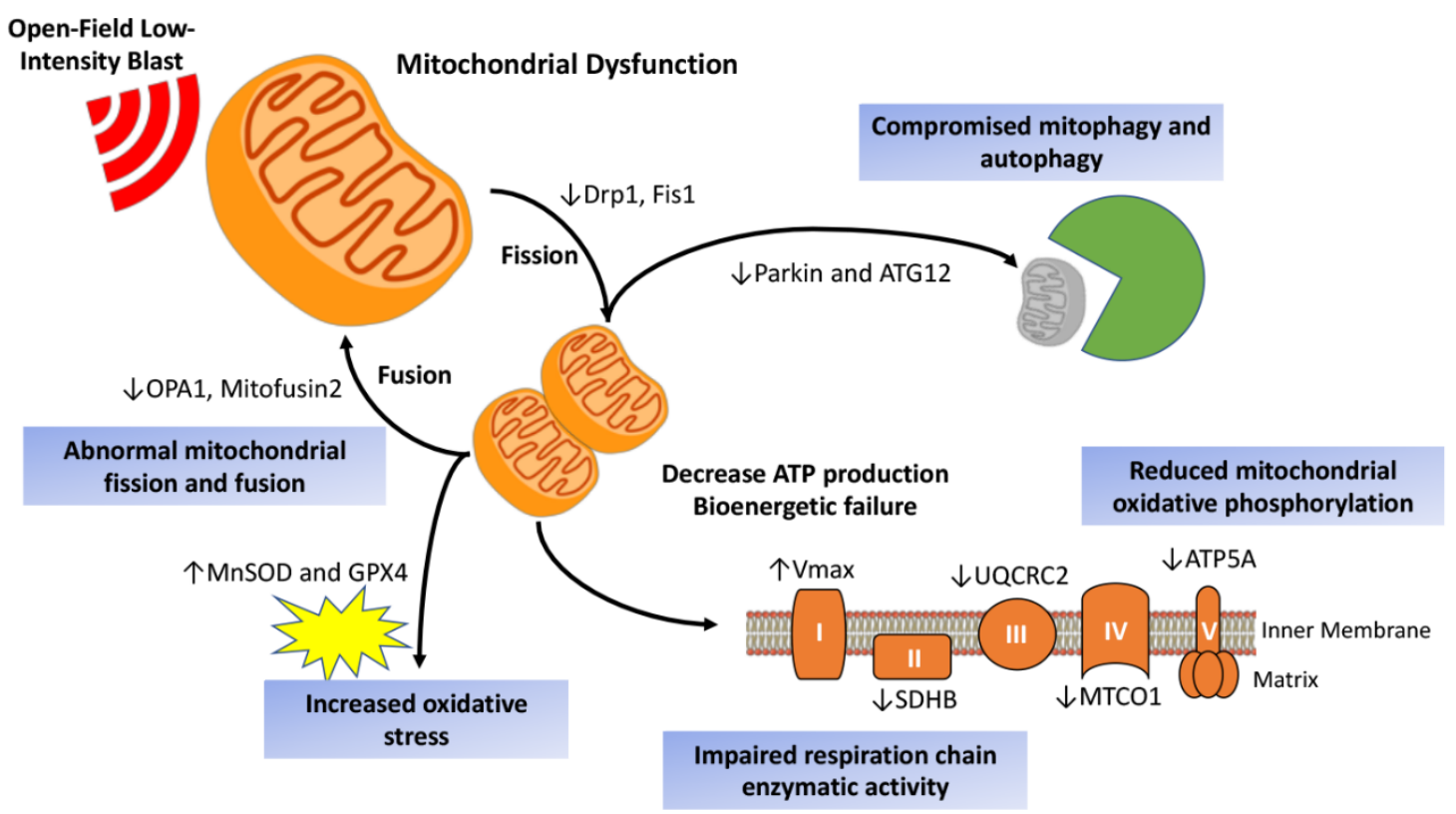

Figure 5.7 A scheme depicting effects of open-field low-intensity primary blast on

\section{mitochondrial dysfunction and canonical pathways.}

Quantitative proteomics and biochemical analysis reveal the mitochondrial dysfunction following low-intensity primary blast. We identified blast-induced mitochondrial damages associated with impaired fission-fusion dynamics, diminished mitophagy, increased oxidative stress, decreased oxidative phosphorylation, and compensated respiration activity.

mTBI usually shows no visible damage using routine imaging techniques. Understanding molecular and ultrastructural mechanisms of LIB induced brain injury requires better identification of specific biomarker surrogates [16, 21]. Proteins, currently including UCH-L1 [18], neuron-specific enolase (NSE) [31], gangliosides / ceramides [32], and S100 calcium-binding protein B (S100B) [33], have been identified as biomarkers for clinical research in patients with severe impact-induced TBI. However, the underlying pathobiology of neurological deficits due to LIB remains unclear. In this 
study, we examined effects of primary LIB on temporal protein changes in both globaland phospho-proteomes by comparing the proteins associated with the cellular components and functional pathways. We first identified a total of 2216 proteins and 459 phosphorylated proteins in brain cortical tissues that were affected by primary blast. Among these, temporal profiles of differentially expressed proteins were characterized at $3 \mathrm{hrs}, 24 \mathrm{hrs}, 7 \mathrm{DPI}$, and 30 DPI after LIB exposure (Figure 5.1). Changes in specific protein and phosphorylated protein targets at $3 \mathrm{hrs}$ after blast remained significantly altered up to $30 \mathrm{DPI}$, such as NDUFA, IMMT, and VDAC1. Some differentially expressed proteins were significantly enriched in the shared cellular components of mitochondria, myelin sheath, actin cytoskeleton, and synapse; as well as in the shared canonical pathways related to mitochondrial dysfunction, axonal / cytoskeletal dysregulation, and synaptic abnormalities in corresponding to their cellular components, and neurodegeneration (Figure 5.2). Distinct proteins with differential expression in the cellular components and signaling pathways were also identified. These findings are consistent with other reports suggesting compromised biological processes, including myelination and cytoskeletal damage caused by moderate or severe TBI [34, 35]. In agreement with our previous observation on LIB-induced damages in mitochondrial ultrastructure [5], results here uncovered changes in mitochondrial injury pathways (Figure 5.3A-B and Table 1). By regulating proteins including GPX4, UQCRC2, IMMT, VDAC1, and STMN1, in both global- and phospho-proteome levels, these signal pathways involve membrane depolarization and biogenesis of mitochondria, as well as fatty acid metabolism and Nrf2 mediated response related to oxidative stress. Further, the IPA disease / function network analysis showed disordered cell-to-cell signaling 
suggesting interactional damage by mitochondrial and other proteins, including IMMT, OPA1, SNAP25, and STMN1 (Figure 5.3C-D). Most importantly, a group of identified mitochondrial related proteins from the proteomic analysis (UQCRC2, IMMT, VDAC1, OPA1) appear to play key roles in regulating mitochondrial integrity (fission and fusion), mitophagy, oxidative phosphorylation, and respiration [36, 37]. The global- and phosphoproteomes suggest that mitochondrial dysfunction peaks between $24 \mathrm{hrs}$ and 7 DPI (Figure 5.3), but some mitochondrial proteins still are persistently altered up to $30 \mathrm{DPI}$. Mitochondrial dysfunction and bioenergetic failure have been implicated in moderate to severe TBI and in certain neurodegenerative diseases [38-40]. Impairment of mitochondrial bioenergetics links to neuronal excitotoxicity, disruption of $\mathrm{Ca}^{2+}$ homeostasis, production of reactive oxygen species (ROS), and ATP depletion [39]. Upregulation of antioxidant MnSOD in TBI study with blast exposure more than $100 \mathrm{kPa}$ has been reported previously [41]. SODs allow conversion of free radicals to hydrogen peroxide and GPX. The present study demonstrated increased MnSOD and GPX4 levels suggesting that LIB may elicit an early occurring compensatory upregulation of mitochondrial antioxidant defenses target (Figure 5.7).

We have characterized the molecular effects of primary LIB by regulating various mitochondrial proteins on its functions including fusion-fission dynamics, oxidative stress, and bioenergetic infrastructure. Of interest is mitochondrial fission protein Drp1 which mediates mitochondrial membrane dynamics and is involved with ROS / RNS and impaired bioenergetics induced synaptic damage and subsequent neuronal loss [42, 43]. Fis1 is involved with mitochondrial fission and recruitment / association of Drp1 [42]. Conversely, mitochondrial fusion is regulated by mitofusin 2 (outer-membrane fusion) 
and OPA1 (inner-membrane fusion) [44]. We first looked at the mitochondrial fusion and fission activities. We found that levels of Drp1, Fis1, OPA1, and mitofusin 2 were significantly reduced to various degrees up to 30 DPI after blast (Figure 5.4D-G). For outer-membrane functions, we identified a significantly reduced level of VDAC1 (voltage-dependent anion channel) which associated with mitochondrial volume regulation (Figure 5.4H). Both mitochondrial fission and fusion play a role to help traffic abnormal mitochondria toward autophagic processing and to maintain normal mitochondrial numbers throughout the neuron. Impaired fission and fusion reportedly interferes with the cell's ability to target dysfunctional mitochondria for autophagic removal [45]. Mitochondrial fission-fusion dynamics has been implicated in neurodegeneration and synaptic plasticity [46]. Our findings on loss of mitochondrial fission and fusion proteins and consequently compromised activities suggest a causal role in the pathogenesis of LIB brain injury and possibly initiation of later neurodegeneration (Figure 5.7).

Structurally damaged mitochondria are most likely bioenergetically impaired [42]. In the nervous system, mitochondria provide a large proportion of ATP required for neuronal function. The oxidative phosphorylation system OXPHOS, embedded in the inner membrane, contains the respiratory chain that utilizes substrates derived from glucose, fatty acids, and amino acids to produce ATP. The electron transport enzymes of the respiratory chain consists of four protein complexes (complex I-IV), three of which (I, III, and IV) couple electron transfer to proton pumping across the mitochondrial inner membrane to generate a transmembrane electrochemical potential [46]. Inefficient electron transfer through complex I-IV interferes with energy metabolism and produces 
of excessive ROS with toxic effects. OXPHOS dysfunction occurs in many neurodegenerative diseases [47]. However, the relationship between OXPHOS and blast injury remain to be fully elucidated [48]. Our previous TEM study demonstrated extensive mitochondrial abnormalities with swollen and clear cristae morphology [5]. In this study, we have identified mitochondrial respiration chain enzyme alterations after LIB exposure by measuring the Vmaxes and subunit levels of key respiratory chain enzymes including complex I and COX, which enzymes contain mtDNA-encoded subunits (Figure 5.5). We observed that multiple OXPHOS protein levels decreased, which could affect cell respiration and ATP production. OXPHOS changes likely also contributed to excessive ROS production (evidenced by the findings of MnSOD and GPX4). Still, it remains to be conclusively determined whether the OXPHOS and respiration enzyme activity are the cause of LIB induced injury or if they are affected by downstream defects in mitochondrial dynamics, structure, and quality control. Further investigation is required to understand these mitochondrial processes.

Mitophagy and autophagy are important cellular processes responsible for breaking down damaged organelles, thereby protecting against accumulation of damaged and aggregated biomolecules. Mitophagy is the selective removal of damaged mitochondria by autophagosomes and lysosomes. Both PINK1 and Parkin are known involved with mitophagy [47]. Upon mitochondrial stress, PINK1 and Parkin remove damaged mitochondria. PINK1, the upstream kinase, accumulates on the mitochondrial surface and recruits the E3 ubiquitin ligase Parkin on site to ubiquitylate substrate proteins which in turn induce the recruitment of receptors following by autophagy [48]. Loss of PINK1 or Parkin after blast disrupts mitophagy from preventing oxidative stress 
damage (shown as the MnSOD data), and are found to be associated with neurodegeneration [49]. Mitochondrial autophagy is also important for mitochondrial quality control. Poor quality mitochondria may enhance oxidative stress and induce cell death [50]. In this study, we identified that primary LIB exposure significantly reduced the Parkin levels at 7 and 30 DPI, and with a trend towards reduced PINK1 (Figure 5.6AC). Parkin substrates including mitofusin 2 and VDAC1, which are embedded in the outer mitochondrial membrane [51], were significantly reduced from 7 to 30 DPI. During autophagy, conjugation of ATG12 to ATG5 is essential for LC3 lipidation and autophagosome formation. In addition, ATG12 functions in diverse processes including mitochondrial fusion and mitochondrial-dependent apoptosis [52]. We observed a significantly decreased level of ubiquitin-like protein ATG12, and a trend towards reduced ULK1 / ATG1 and LC3B after LIB exposure (Figure 5.6D). These results suggest a decreased level of ATG12-ATG5 covalent binding which further leads to insufficient autophagosome maturation and subsequent attenuation of autophagic flux [53]. Together, our findings indicated that LIB resulted in compromised mitophagy and autophagy (Figure 5.7).

Mitochondrial damage after blast may further lead to impaired neuronal cell function and contribute to the development or progression of neurodegeneration [10, 38]. Defects in mitochondrial bioenergetics and associated metabolic function are known to link with alterations of mitostatic processes, including mitochondrial clearance / dynamics, and these changes would impact myelination, axonal transport, and synaptic regulation [54]. From our previous report and others, myelin sheaths defects exhibit multiple features, including sheath disruption, detachment from the axonemal, splitting of 
the myelin lamellae, and dense degeneration with vacuole inclusions within the myelin lamellae $[5,55]$. This study identified canonical pathways associated with axonal guidance, regulation of cytoskeleton, and synaptic vesicle cycle regulation (Table 2-3). Interestingly, we observed downregulation of SNAP 25 and upregulation of STMN1 phosphorylation after LIB exposure. It has been reported that STMN1 phosphorylation regulates microtubule disassembly affecting axonal injury after blast at $74.5 \mathrm{kPa}[56]$. SNAP 25, a component of synapses, is also important to synaptic functions, and is altered in diffuse axonal injury [57].

Overall, our findings demonstrate that primary LIB exposure at $46.6 \mathrm{kPa}$ causes striking mitochondrial dysfunction. Resulting dynamic global- and phospho-proteome alterations and underlying mechanisms involved with mitochondrial dysfunction, oxidative stress, axonal / cytoskeleton regulation are further revealed in the LIB exposed cortical brain tissues. It is a logical extension on the effects of blast intensities that LIBinduced ultrastructural mitochondrial damage leading to prolonged dysregulation of cytoskeletal and synaptic proteins. Future studies with isolated mitochondrial proteome will permit further characterization of LIB-induced mitochondrial changes in related to mitochondrial permeability, mitochondrial membrane potential, and ATP production leading to neurodegeneration. In addition, LIB induced injury subjected to other brain regions, such as hippocampus, striatum, and cerebellum, will be surveyed. The chronic effects of LIB induced brain injury will also be investigated [7]. Nevertheless, these studies have documented the key relationships of blast exposures to oxidative stress and mitochondrial dysfunction. Insights into the acute pathogenesis of LIB-induced mTBI may indicate links between early biochemical changes and later brain degeneration. It 
remains to be determined whether highly specific target characterization at molecular levels may enable more sensitive biomarker development, as well as particular target proteins and/or time lines for prevention and treatment of primary blast injuries. 


\subsection{REFERENCES}

[1] S.T. DeKosky, M.D. Ikonomovic, S. Gandy, Traumatic Brain Injury -- Football, Warfare, and Long-Term Effects., N Engl J Med 363(14) (2010) 1293-1296.

[2] B.M. Aertker, S. Bedi, C.S. Cox Jr, Strategies for CNS repair following TBI, Exp Neurol 275 (2016) 411-426.

[3] R.G. DePalma, D.G. Burris, H.R. Champion, M.J. Hodgson, Blast injuries, N Engl J Med 352(13) (2005) 1335-42.

[4] I. Cernak, L.J. Noble-Haeusslein, Traumatic brain injury: an overview of pathobiology with emphasis on military populations, J Cereb Blood Flow Metab 30(2) (2010) 255-66.

[5] H. Song, L.M. Konan, J. Cui, C.E. Johnson, M. Langenderfer, D. Grant, T. Ndam, A. Simonyi, T. White, U. Demirci, D.R. Mott, D. Schwer, G.K. Hubler, I. Cernak, R.G. DePalma, Z. Gu, Ultrastructural brain abnormalities and associated behavioral changes in mice after low-intensity blast exposure, Behav Brain Res 347 (2018) 148-157.

[6] H. Song, J. Cui, A. Simonyi, C.E. Johnson, G.K. Hubler, R.G. DePalma, Z. Gu, Linking blast physics to biological outcomes in mild traumatic brain injury: Narrative review and preliminary report of an open-field blast model, Behav Brain Res 340 (2018) $147-158$.

[7] H. Song, L.M. Konan, J. Cui, C.E. Johnson, G.K. Hubler, R.G. DePalma, Z. Gu, Nanometer ultrastructural brain damage following low intensity primary blast wave exposure, Neural Regen Res 13(9) (2018) 1516-1519.

[8] M. Chen, H. Song, J. Cui, C. Johnson, G. Hubler, R.G. DePalma, Z. Gu, X. Weiming, Proteomic profiling of mouse brains exposed to blast-induced mild traumatic brain injury 
reveals changes in axonal proteins and phosphorylated tau, J Alzheimers Dis Prepress(Pre-press) (2018) 1-23.

[9] M.T. Lin, M.F. Beal, Mitochondrial dysfunction and oxidative stress in neurodegenerative diseases, Nature 443(7113) (2006) 787.

[10] J.B. Hiebert, Q. Shen, A.R. Thimmesch, J.D. Pierce, Traumatic brain injury and mitochondrial dysfunction, Am J Med Sci 350(2) (2015) 132-138.

[11] A. Bertholet, T. Delerue, A. Millet, M. Moulis, C. David, M. Daloyau, L. ArnaunePelloquin, N. Davezac, V. Mils, M. Miquel, Mitochondrial fusion/fission dynamics in neurodegeneration and neuronal plasticity, Neurobiol Dis 90 (2016) 3-19.

[12] M. Xiao, H. Zhong, L. Xia, Y. Tao, H. Yin, Pathophysiology of mitochondrial lipid oxidation: role of 4-hydroxynonenal (4-HNE) and other bioactive lipids in mitochondria, Free Radic Biol Med 111 (2017) 316-327.

[13] A.L. Swan, A. Mobasheri, D. Allaway, S. Liddell, J. Bacardit, Application of machine learning to proteomics data: classification and biomarker identification in postgenomics biology, Omics 17(12) (2013) 595-610.

[14] G.A. Sarkis, M.D. Mangaonkar, A. Moghieb, B. Lelling, M. Guertin, H. Yadikar, Z. Yang, F. Kobeissy, K.K. Wang, The application of proteomics to traumatic brain and spinal cord injuries, Curr Neurol Neurosci Rep 17(3) (2017) 23.

[15] D.K. Kim, J. Park, D. Han, J. Yang, A. Kim, J. Woo, Y. Kim, I. Mook-Jung, Molecular and functional signatures in a novel Alzheimer's disease mouse model assessed by quantitative proteomics, Mol Neurodegener 13(1) (2018) 2. 
[16] F.H. Kobeissy, A.K. Ottens, Z. Zhang, M.C. Liu, N.D. Denslow, J.R. Dave, F.C. Tortella, R.L. Hayes, K.K. Wang, Novel differential neuroproteomics analysis of traumatic brain injury in rats, Mol Cell Proteomics 5(10) (2006) 1887-1898. [17] T. Liu, W.-J. Qian, M.A. Gritsenko, W. Xiao, L.L. Moldawer, A. Kaushal, M.E. Monroe, S.M. Varnum, R.J. Moore, S.O. Purvine, High dynamic range characterization of the trauma patient plasma proteome, Mol Cell Proteomics 5(10) (2006) 1899-1913. [18] A.M. Boutté, C. Yao, F. Kobeissy, X.C. May Lu, Z. Zhang, K.K. Wang, K. Schmid, F.C. Tortella, J.R. Dave, Proteomic analysis and brain-specific systems biology in a rodent model of penetrating ballistic-like brain injury, Electrophoresis 33(24) (2012) 3693-3704.

[19] M.E. Peters, V. Rao, K.T. Bechtold, D. Roy, H.I. Sair, J.-M. Leoutsakos, R. DiazArrastia, R.D. Stevens, D.S. Batty Jr, H. Falk, Head injury serum markers for assessing response to trauma: design of the HeadSMART study, Brain Inj 31(3) (2017) 370-378. [20] M. Ning, D.A. Sarracino, A.T. Kho, S. Guo, S.-R. Lee, B. Krastins, F.S. Buonanno, J.A. Vizcaíno, S. Orchard, D. McMullin, X. Wang, E.H. Lo, Proteomic Temporal Profile of Human Brain Endothelium After Oxidative Stress, Stroke 42(1) (2011) 37-43. [21] A. Saljo, B. Svensson, M. Mayorga, A. Hamberger, H. Bolouri, Low-level blasts raise intracranial pressure and impair cognitive function in rats, J Neurotrauma 26(8) (2009) 1345-1353.

[22] J. Villén, S.P. Gygi, The SCX/IMAC enrichment approach for global phosphorylation analysis by mass spectrometry, Nat Protoc 3(10) (2008) 1630.

[23] F.-K. Huang, G. Zhang, K. Lawlor, A. Nazarian, J. Philip, P. Tempst, N. Dephoure, T.A. Neubert, Deep coverage of global protein expression and phosphorylation in breast 
tumor cell lines using TMT 10-plex isobaric labeling, J Proteome Res 16(3) (2017) 11211132.

[24] J.E. Elias, S.P. Gygi, Target-decoy search strategy for increased confidence in largescale protein identifications by mass spectrometry, Nat Methods 4(3) (2007) 207. [25] X. Wang, Q. Zhang, R. Bao, N. Zhang, Y. Wang, L. Polo-Parada, A. Tarim, A. Alemifar, X. Han, H.M. Wilkins, Deletion of Nampt in Projection Neurons of Adult Mice Leads to Motor Dysfunction, Neurodegeneration, and Death, Cell Rep 20(9) (2017) 2184-2200.

[26] Z. Qu, F. Meng, R.D. Bomgarden, R.I. Viner, J. Li, J.C. Rogers, J. Cheng, C.M. Greenlief, J. Cui, D.B. Lubahn, G.Y. Sun, Z. Gu, Proteomic quantification and sitemapping of S-nitrosylated proteins using isobaric iodoTMT reagents, J Proteome Res 13(7) (2014) 3200-11.

[27] H. Chen, P.C. Boutros, VennDiagram: a package for the generation of highlycustomizable Venn and Euler diagrams in R, BMC Bioinformatics 12(1) (2011) 35. [28] G. Yu, L.-G. Wang, Y. Han, Q.-Y. He, clusterProfiler: an R package for comparing biological themes among gene clusters, Omics 16(5) (2012) 284-287. [29] W. Walter, F. Sánchez-Cabo, M. Ricote, GOplot: an R package for visually combining expression data with functional analysis, Bioinformatics 31(17) (2015) 29122914.

[30] H. Song, Y. Lu, Z. Qu, V.V. Mossine, M.B. Martin, J. Hou, J. Cui, B.A. Peculis, T.P. Mawhinney, J. Cheng, Effects of aged garlic extract and FruArg on gene expression and signaling pathways in lipopolysaccharide-activated microglial cells, Sci Rep 6 (2016) 35323. 
[31] S. Mondello, U. Muller, A. Jeromin, J. Streeter, R.L. Hayes, K.K. Wang, Bloodbased diagnostics of traumatic brain injuries, Expert Rev Mol Diagn 11(1) (2011) 65-78. [32] A.S. Woods, B. Colsch, S.N. Jackson, J. Post, K. Baldwin, A. Roux, B. Hoffer, B.M. Cox, M. Hoffer, V. Rubovitch, Gangliosides and ceramides change in a mouse model of blast induced traumatic brain injury, ACS Chem Neurosci 4(4) (2013) 594-600.

[33] A. Rodríguez-Rodríguez, J.J. Egea-Guerrero, A. León-Justel, E. Gordillo-Escobar, J. Revuelto-Rey, Á. Vilches-Arenas, A. Carrillo-Vico, J.M. Domínguez-Roldán, F. MurilloCabezas, J.M. Guerrero, Role of S100B protein in urine and serum as an early predictor of mortality after severe traumatic brain injury in adults, Clin Chim Acta 414 (2012) 228233.

[34] Y.C. Yap, A.E. King, R.M. Guijt, T. Jiang, C.A. Blizzard, M.C. Breadmore, T.C. Dickson, Mild and repetitive very mild axonal stretch injury triggers cystoskeletal mislocalization and growth cone collapse, PloS One 12(5) (2017) e0176997.

[35] H. Song, S. Fang, J. Gao, J. Wang, Z. Cao, Z. Guo, Q. Huang, Y. Qu, H. Zhou, J. Yu, Quantitative Proteomic Study Reveals Up-Regulation of cAMP Signaling PathwayRelated Proteins in Mild Traumatic Brain Injury, J Proteome Res 17(2) (2018) 858-869. [36] W. Dai, H.-1. Cheng, R.-q. Huang, Z. Zhuang, J.-X. Shi, Quantitative detection of the expression of mitochondrial cytochrome $\mathrm{c}$ oxidase subunits mRNA in the cerebral cortex after experimental traumatic brain injury, Brain Res 1251 (2009) 287-295. [37] V. Di Pietro, G. Lazzarino, A.M. Amorini, S. Signoretti, L.J. Hill, E. Porto, B. Tavazzi, G. Lazzarino, A. Belli, Fusion or Fission: The Destiny of Mitochondria In Traumatic Brain Injury of Different Severities, Sci Rep 7(1) (2017) 9189. 
[38] R.H. Swerdlow, Mitochondria and Mitochondrial Cascades in Alzheimer's Disease, J Alzheimers Dis (Preprint) (2017) 1-14.

[39] J. Kulbe, E. Hall, Chronic traumatic encephalopathy-integration of canonical traumatic brain injury secondary injury mechanisms with tau pathology, Prog Neurobiol 158 (2017) $15-44$.

[40] J.L. Harmon, W.S. Gibbs, R.M. Whitaker, R.G. Schnellmann, D.L. Adkins, Striatal Mitochondrial Disruption following Severe Traumatic Brain Injury, J Neurotrauma 34(2) (2017) 487-494.

[41] B.R. Huber, J.S. Meabon, T.J. Martin, P.D. Mourad, R. Bennett, B.C. Kraemer, I. Cernak, E.C. Petrie, M.J. Emery, E.R. Swenson, Blast exposure causes early and persistent aberrant phospho-and cleaved-tau expression in a murine model of mild blastinduced traumatic brain injury, J Alzheimers Dis 37(2) (2013) 309-323.

[42] M.J. Barsoum, H. Yuan, A.A. Gerencser, G. Liot, Y. Kushnareva, S. Gräber, I. Kovacs, W.D. Lee, J. Waggoner, J. Cui, Nitric oxide-induced mitochondrial fission is regulated by dynamin-related GTPases in neurons, EMBO J 25(16) (2006) 3900-3911. [43] D.-H. Cho, T. Nakamura, J. Fang, P. Cieplak, A. Godzik, Z. Gu, S.A. Lipton, Snitrosylation of Drp1 mediates $\beta$-amyloid-related mitochondrial fission and neuronal injury, Science 324(5923) (2009) 102-105.

[44] J.E. Selfridge, E. Lezi, J. Lu, R.H. Swerdlow, Role of mitochondrial homeostasis and dynamics in Alzheimer's disease, Neurobiol Dis 51 (2013) 3-12.

[45] G. Twig, B. Hyde, O.S. Shirihai, Mitochondrial fusion, fission and autophagy as a quality control axis: the bioenergetic view, Biochim Biophys Acta 1777(9) (2008) 10921097. 
[46] J. Balog, S.L. Mehta, R. Vemuganti, Mitochondrial fission and fusion in secondary brain damage after CNS insults, J Cereb Blood Flow Metab 36(12) (2016) 2022-2033. [47] T.N. Nguyen, B.S. Padman, M. Lazarou, Deciphering the molecular signals of PINK1/Parkin mitophagy, Trends Cell Biol 26(10) (2016) 733-744.

[48] N. Matsuda, S. Sato, K. Shiba, K. Okatsu, K. Saisho, C.A. Gautier, Y.-s. Sou, S. Saiki, S. Kawajiri, F. Sato, PINK1 stabilized by mitochondrial depolarization recruits Parkin to damaged mitochondria and activates latent Parkin for mitophagy, J Cell Biol 189(2) (2010) 211-221.

[49] K. Itoh, K. Nakamura, M. Iijima, H. Sesaki, Mitochondrial dynamics in neurodegeneration, Trends Cell Biol 23(2) (2013) 64-71.

[50] J. Zhang, Autophagy and mitophagy in cellular damage control, Redox Biol 1(1) (2013) 19-23.

[51] R.J. Youle, D.P. Narendra, Mechanisms of mitophagy, Nat Rev Mol Cell Biol 12(1) (2011) 9 .

[52] L. Radoshevich, L. Murrow, N. Chen, E. Fernandez, S. Roy, C. Fung, J. Debnath, ATG12 conjugation to ATG3 regulates mitochondrial homeostasis and cell death, Cell 142(4) (2010) 590-600.

[53] C. Sarkar, Z. Zhao, S. Aungst, B. Sabirzhanov, A.I. Faden, M.M. Lipinski, Impaired autophagy flux is associated with neuronal cell death after traumatic brain injury, Autophagy 10(12) (2014) 2208-2222.

[54] T. Misgeld, T.L. Schwarz, Mitostasis in neurons: maintaining mitochondria in an extended cellular architecture, Neuron 96(3) (2017) 651-666. 
[55] J.O. Ojo, C. Bachmeier, B.C. Mouzon, R. Tzekov, M. Mullan, H. Davies, M.G. Stewart, F. Crawford, Ultrastructural Changes in the White and Gray Matter of Mice at Chronic Time Points After Repeated Concussive Head Injury, J Neuropathol Exp Neurol 74(10) (2015) 1012-35.

[56] G.A. Elder, N.P. Dorr, R. De Gasperi, M.A. Gama Sosa, M.C. Shaughness, E. Maudlin-Jeronimo, A.A. Hall, R.M. McCarron, S.T. Ahlers, Blast exposure induces posttraumatic stress disorder-related traits in a rat model of mild traumatic brain injury, $\mathrm{J}$ Neurotrauma 29(16) (2012) 2564-75.

[57] P. Zhang, S. Zhu, Y. Li, M. Zhao, M. Liu, J. Gao, S. Ding, J. Li, Quantitative proteomics analysis to identify diffuse axonal injury biomarkers in rats using iTRAQ coupled LC-MS/MS, J Proteom 133 (2016) 93-99. 


\section{CHAPTER 6 PROTEOMIC PROFILING OF MOUSE BRAINS EXPOSED TO BLAST-INDUCED MILD}

TRAUMATIC BRAIN INJURY REVEALS CHANGES IN

AXONAL PROTEINS AND PHOSPHORYLATED TAU

\subsection{ABSTRACT}

Alzheimer's disease (AD), the most prevalent form of dementia, is characterized by two pathological hallmarks: Tau-containing neurofibrillary tangles and amyloid $\beta$ protein (A $\beta$ )-containing neuritic plaques. The goal of this study is to understand mild traumatic brain injury (mTBI)-related brain proteomic changes and tau-related biochemical adaptations that may contribute to $\mathrm{AD}-$ like neurodegeneration. We found that both phosphorylated tau (p-tau) and the ratio of p-tau/tau were significantly increased in brains of mice collected at 3 and 24 hours after exposure to $82-\mathrm{kPa}$ low-intensity open-field blast. Neurological deficits was observed in animals at 24 hours and 7 days after the blast using Simple Neuroassessment of Asymmetric imPairment (SNAP) test, and axon/dendrite degeneration at 7 days was revealed by silver staining. Liquid chromatography/mass spectrometry (LC/MS) was used to analyze brain tissue labeled with isobaric mass tags for relative protein quantification. The results from the proteomics and bioinformatic analysis illustrated the alterations of axonal and synaptic proteins in related pathways, including but not being limited to substantia nigra degeneration, cortical cytoskeleton organization and synaptic vesicle exocytosis, suggesting a potential axonal damage caused by blast-induced mTBI. Among altered 
proteins found in brains suffering blast, microtubule-associated protein $1 \mathrm{~B}$, stathmin, neurofilaments, actin binding proteins, myelin basic protein, calcium/calmodulindependent protein kinase and synaptotagmin I were representative ones involved in altered pathways elicited by mTBI. Therefore, TBI induces elevated phosphor-tau, a pathological feature found in brains of $\mathrm{AD}$, and altered a number of neurophysiological processes, supporting the notion that blast-induced $\mathrm{mTBI}$ as a risk factor contributes to AD pathogenesis. LC/MS-based profiling has presented candidate target/pathways that could be explored for future therapeutic development.

\subsection{INTRODUCTION}

Mild traumatic brain injury (mTBI) results in significant morbidity and mortality throughout the world. Overlapping neuropathological features of patients suffering Alzheimer's disease (AD) or traumatic brain injury (TBI) suggest that pathological proteins for these two conditions may interact $[1,2]$. In $\mathrm{AD}$, widespread neuronal loss is associated with the pathological hallmarks of neurofibrillary tangles composed of phosphorylated tau and neuritic plaques composed of amyloid $\beta$ protein $(\mathrm{A} \beta)$. Animal brains suffering TBI exhibit tau pathology upon following controlled cortical impact (CCI), and these transgenic mice exhibit dramatic increases in levels of tau [1]. Several studies have associated TBI with a significantly increased risk of the development of late neurodegenerative diseases, such as Alzheimer's and Parkinson's disease [3]. Soldiers exposed to even a single blast, can develop behavioral and psychiatric problems within a year following injury [4]. Even a single TBI may induce long-term neuropathological changes akin to those found in neurodegenerative disease and associated with the later 
development of syndromes of cognitive impairment such as $\mathrm{AD}$ [5]. In humans, repetitive impact-induced mTBI leads to chronic traumatic encephalopathy (CTE) with increased tau pathology and other neurodegenerative changes in the brain [6]. CTE has been observed in Veterans exposed to explosive blast [7]. Clinical reports describe postconcussive stress and depression symptoms along with worse cognitive performance in subjects with blast-induced mTBI, both in short- (0-7 days) and long-term (6 months to 5 years) follow-up exams after the blast [8-10]. Additional reports confirm that mTBI is positively associated with depression and increased anxiety levels [11].

Among the several mechanisms that have been proposed as possible explanations for these associations, two prime events occurring in ongoing blast injury are neuroinflammation and deposition of abnormal microtubule proteins such as tau [12]. Mutations in the tau gene cause frontotemporal dementia with Parkinsonism linked to chromosome 17 (FTDP-17) [13]. Transgenic mice expressing mutant tau show close association with tau mutation-containing, neurofibrillary tangle (NFT) formation, and neurodegeneration [14-16]. Tau protein has been used as a marker for axonal damage, and tau levels in cerebrospinal fluid (CSF) reflect these changes in the central nervous system (CNS), especially, total tau protein levels (t-tau), phosphorylated tau (p-tau) and the ratio of $\mathrm{A} \beta 42 /$ tau have been explored as potential markers for $\mathrm{AD}$ [17-19]. Other related proteins include neurofilament light chain (NfL), which was found to be increased in CSF and blood of patients with AD or tauopathies. In animals, blocking $\mathrm{A} \beta$ lesions attenuates the NfL increase [20]. These proteins may affect the formation of $A \beta /$ tau pathologies. 
Proteomic research $[21,22]$ using brain tissues or biofluids from either patients or animal models have made it feasible to identify a large number of proteins associated with mTBI $[23,24]$. MS-based proteomics possess the advantage of having no requirement of prior knowledge of the proteins being identified, allowing for unbiased, hypothesis-free biomarker discovery in complex biological samples such as plasma and brain tissue extracts. Therefore, MS-based proteomic approach can be used to unravel novel biological processes underlying TBI, neurodegeneration and repair, or to represent complex protein-protein interactions during disease progression. However, MS-based proteomic analysis has not been performed on samples derived from animals exposed to open field blast-induced mTBI.

In this study, we use proteomic profiling of mouse brains suffering blast-induced mTBI to explore contribution of mTBI toward AD pathologic process. Tau associated biological processes were found to be differentially regulated in blast-induced mTBI mouse models, and levels of phospho-tau were increased in brains of mice collected at 3 and 24 hours (hr) after the exposure to blast. Behavioral and pathological alterations were observed in animals exposed to blast. We employed tandem mass tag (TMT)-labelling in conjunction with liquid chromatography-tandem mass spectrometry (LC-MS/MS) approach to investigate differentially expressed proteins in mouse brain samples after exposed to a single low-intensity open-field blast for the purpose of discovering candidate proteins associated with blast-induced mTBI and AD pathology. Differentially expressed proteins were analyzed using bioinformatics tools DAVID and Metacore to determine if functional relationships were altered in mice exposed to blast. This approach allows us to evaluate effect of blast on mice by understanding the proteomic profiles of 
brain tissue as a way of identifying potential molecular signatures of pathological alteration that may contribute to $\mathrm{AD}$ pathogenesis.

\subsection{METHOD}

\subsubsection{Materials}

Unless indicated, all reagents used for biochemical methods and cell culture preparation were purchased from Sigma-Aldrich (St Louis, MO, USA). Reagents for BCA protein assay and sample preparation for LC-MS/MS analysis were purchased from Thermo Scientific (Rockford, IL, USA). TMT reagent six-plex and ten-plex kits were available commercially (Thermo Scientific Pierce, Rockford, IL, USA).

\subsubsection{Open-field primary blast injury in mice}

Male C57BL/6J mice (The Jackson Laboratory, Bar Harbor, ME), at 2 months old, were housed with a 12-hr light/dark cycle and given unrestricted access to food and water. Open-field blast exposures were conducted at the Missouri University of Science \& Technology and were recently reported $[25,26]$. Briefly, for the experimental setting of open-field low-intensity blast injury with the modification as described here, mice were placed in animal holders in prone position at 2.15-meter (m) distance away from detonation of $350 \mathrm{~g} \mathrm{C} 4$ explosive (both $1 \mathrm{~m}$ above ground) generating a magnitude of 82 $\mathrm{kPa}$ [e.g., 11.9 pounds per square inch (PSI)] peak overpressure and maximal impulse at $71 \mathrm{kPa}(10.3 \mathrm{PSI}) \mathrm{x} \mathrm{ms}$. The Sham group underwent identical procedures as blast groups only without blast exposure. Following blast wave exposure, animals were removed from the platform and returned to the original cage. After recovery from anesthesia, mice were 
able to spontaneously move and continuously being monitored for at least 15-30 min. Animals were allowed access to food and water ad libitum before and after blast exposure. Mice were randomly assigned into experimental or sham control groups which were double-blinded to investigators analyzing experimental outcomes. All procedures were in accordance with the University of Missouri approved protocols for the Care and Use of Laboratory Animals and ARRIVE guidelines.

\subsubsection{Behavioral test}

Behavioral test using Simple Neuroassessment of Asymmetric imPairment (SNAP) after blast exposure was used to assess the neurological status of coordination, motor strength, proprioception, gait and mental responses. The tests examined interaction, cage grasp, visual placing, pacing/circling, gait/posture, head tilt, visual field, and baton as we previously described [25, 27].

\subsubsection{Brain collection and pathological examination}

There were total thirty seven mice in the present study, in which thirty two mice were randomly divided into four groups at $3 \mathrm{hr}(\mathrm{n}=4), 24 \mathrm{hrs}(\mathrm{n}=9), 7$ days $(\mathrm{n}=11), 30$ days $(n=4)$ and 15 weeks $(n=4)$ after a single $82-k P a$ low-intensity blast exposure and five mice as the sham control group [25, 28, 29]. Mice were euthanized using overdoing of isoflurane and cerebral cortex regions were dissected from the blast-exposed mouse brain. Silver staining and quantitative analyses were performed as previously described $[25,30]$. The FD NeuroSilver Kit II was used for silver staining according to the manufacturer's instructions. Each batch of the experiments was included the brain 
sections from different groups to avoid variability of the staining intensity. Tiff images of sections with the defined coordinates for stereological analysis were captured containing the regions of interests of the corpus callosum and entorhinal cortex (bregma 0.14 to 0.02 $\mathrm{mm}$ ), cerebral peduncle (bregma -1.94 to $-2.06 \mathrm{~mm}$ ), and fornix (bregma 0.14 to 0.02 $\mathrm{mm})$, based on the mouse brain atlas. These images were exported and analyzed with ImageJ software (NIH, Bethesda, MD). Background (gold color) was subtracted from each image using the color splitter and image calculator functions, so that only the grayblack silver deposits were visible. Densitometric analysis of silver staining was then performed using ImageJ. Serial sections were averaged from each animal to produce a final silver staining value. The intensity of silver staining was expressed in arbitrary units, ranging from 0 (minimum) to 255 (maximum).

\subsubsection{Preparation of brain tissue for MS}

Sample lysis buffer (2\% SDS, 0.5 Mtetraethylammonium bicarbonate (TEAB), protease inhibitor cocktail) were added to each brain tissue and then homogenized by TissueLyser LT (Qiagen, Valencia, CA). Tissue homogenates were centrifuged at $17,000 \times \mathrm{g}$, for 20 min at $4{ }^{\circ} \mathrm{C}$. The supernatant was transferred into a new vial for protein concentration measurement by BCA assay. Preparation of tryptic peptides for TMT 10-plex labelling was carried out according to the manufacturer's instructions. Briefly $100 \mu \mathrm{g}$ protein from each sample was transferred into a new vial and adjusted to a final volume of $100 \mu \mathrm{L}$ with TEAB and reduced with tris (2-carboxyethyl) phosphine (TCEP) at $55^{\circ} \mathrm{C}$ for 1 hour, and then alkylated with iodoacetamide for 30 minutes in the dark. Methanol-chloroform precipitation was performed prior to protease digestion. In brief, four parts methanol was 
added to each sample and vortexed, then one part chloroform was added to the sample and vortexed, and three parts water was added to the sample and vortexed. The sample was centrifuged at $14,000 \mathrm{~g}$ for $4 \mathrm{~min}$ at room temperature and the aqueous phase was removed. The organic phases with protein precipitate at the surface was subsequently washed twice with four parts methanol and centrifuged with supernatant being removed subsequently. After air-drying, precipitated protein pellets were re-suspend with $100 \mu \mathrm{L}$ of 50mM TEAB and digested with trypsin overnight at $37^{\circ} \mathrm{C}$.

\subsubsection{TMT-labeling and sample clean up}

TMT enables relative quantitation of proteins present in multiple samples by labeling peptides with isobaric stable isotope tags that fragment upon collision-induced dissociation into reporter ions used for quantitation. In this study, TMT ten tags for ten samples (blast samples and controls) were analyzed simultaneously, avoiding run-to-run variation. Tryptic digested peptides from brain samples were labeled with TMT 10-plex reagents (Thermo Fisher) according to manufacturer's instructions. Briefly, the TMT reagents $(0.8 \mathrm{mg})$ were dissolved in $41 \mu \mathrm{L}$ of anhydrous acetonitrile. Aliquots of samples were incubated with TMT reagents for 1 hour at room temperature. The reactions were quenched by $8 \mu \mathrm{L}$ of $5 \%$ hydroxylamine solution and reacted for $15 \mathrm{~min}$. The combined TMT labelled samples were dried under SpeedVac, and then reconstituted by dilute trifluoroacetic acid solution followed by desalting by Oasis HLB 96-well $\mu$ Elutionplate (Waters) prior to LC-MS/MS analysis.

\subsubsection{Phospho-protein enrichment and analysis}


For enrichment of phospho peptides, samples were processed using the Fe-NTA phospho peptide enrichment kit and followed by graphite spin columns according to the manufacturer's instructions (Thermo Scientific Pierce) before LC-MS/MS analysis.

\subsubsection{LC-MS/MS analysis}

LC MS/MS was performed on a Q Exactive Orbitrap Mass Spectrometer (Thermo Fisher Scientific) coupled with a Dionex ultimate 3000 HPLC system equipped with a nano-ES ion source. The TMT labelled peptides were separated on a C18 reverse-phase capillary column (PepMap, $75 \mu \mathrm{m} \times 150 \mathrm{~mm}$, Thermo Fisher) with linear gradients of $2-35 \%$ acetonitrile in $0.1 \%$ formic acid, at a constant flow rate of $300 \mathrm{~nL} / \mathrm{min}$ for $220 \mathrm{~min}$. The instrument was operated in the positive-ion mode with the ESI spray voltage set at 1.8 $\mathrm{kV}$. Afullscan MS spectra (300-1800 m/z) was acquired in the Orbitrap at a mass resolution of 70,000 with an automatic gain control target (AGC) of 3e6. Fifteen peptide ions showing the most intense signal from each scan were selected for higher energy collision-induced dissociation (HCD)-MS/MS analysis (normalized collision energy 32) in the Orbitrap at a mass resolution of 35,000 and AGC value of 1e5. Maximal filling times were $100 \mathrm{~ms}$ in full scans and $120 \mathrm{~ms}$ (HCD) for the MS/MS scans. Ions with unassigned charge states and singly charged species were rejected. The dynamic exclusion was set to $50 \mathrm{~s}$ and a relative mass window of $10 \mathrm{ppm}$. The data were acquired using ThermoXcalibur 3.0.63. The phospho peptides were analyzed under the same conditions as above, other than the separation which was done with linear gradients of 1$30 \%$ acetonitrile in $0.1 \%$ formic acid, and full scan MS ranging 300-2000 m/z with NCE at 25 . 


\subsubsection{Protein identification and quantification}

Raw data were processed using Proteome Discoverer (Version 2.1, Thermo Fisher Scientific). Data were searched against the mus musculus Universal Protein Resource sequence database (UniProt, August, 2013). The searches were performed with the following guidelines: trypsin digestion with two missed cleavage allowed; fixed modification, carbamidomethyl of cysteine; variable modification, oxidation of methionine, TMT 10plex (peptide labeled) for $\mathrm{N}$ terminus and Lys; MS tolerance, 5 ppm; MS/MS tolerance, $0.02 \mathrm{Da}$; false discovery rate (FDR) at peptide and protein levels, $<$ 0.01 ; and required peptide length, $\geq 6$ amino acids. Phosphorylation of serine, threonine and tyrosine was defined as variable modification. Protein grouping was enabled, meaning if one protein were equal to or completely contained within the set of peptides of another protein, these two proteins were put into the same protein group. At least one unique peptide per protein group was required for identifying proteins. The relative protein abundance ratios (fold changes) between blasted and shamed groups were calculated. The changes in protein levels were considered significant if fold change was $>1.2$ (up-regulated) or $<0.9$ (down-regulated), and the p-value is less than 0.05 in two independent experiments.

\subsubsection{Quantification of tau and phospho-tau by ELISA}

Tau and p-tau peptide at Thr residue 181 were quantified by ELISA [31]. The capture antibody for tau is BT-2. To capture specific phosphorylated tau at Thr residue 181, antibody AT270 was used. The detecting antibody is the biotinylated HT7 that recognizes 
residue 159-163 of tau. All three tau antibodies were purchased from Thermo Scientific (Rockford, IL). ELISA was performed using Meso Scale Discovery platform (MSD, Gaithersburg, MD). The internal calibrators of p-tau and tau were purchased from Meso Scale Discovery.

\subsubsection{Data analysis}

An enrichment analysis was performed by submitting the UniProt accession numbers containing the list of differentially regulated proteins to open-source software Database for Annotation, Visualization, and Integrated Discovery (DAVID) v6.7 [32, 33]. DAVID is able to extract biological features/meanings associated with large gene lists. The gene names of the proteins were entered to analyze GO biological process (GOBP), GO molecular function (GOMF), and GO cellular components (GOCC) and KEGG pathways. Statistically significant differences $(p$-value $<0.05)$ were identified using an EASE score (a modification of Fisher's exact test), which was provided by the DAVID gene bioinformatics online resource. The cellular and molecular process networks of the dysregulated proteins were also analyzed using Metacore. Metacore uses these proteins and their identifiers to navigate the curated literature database and extract the biologically relevant information among the candidate proteins. Associated biofunctions were generated, along with a score representing the log probability of a particular process network.

\subsection{RESULTS}

\subsubsection{Increase of phospho-tau in brains of mice at 3 and 24 hours after the blast}


In this study, we analyzed the mouse brain samples at $3 \mathrm{hr}, 24 \mathrm{hr}, 30$ days and 15 weeks after the blast. We applied ELISA to quantify levels of tau and phospho-tau in the same brain tissue later used for LC-MS/MS analysis. We found a significant increase of phospho-tau in mouse brains collected at 3 and 24 hours after the blast, while a reduction of tau was also observed in the same samples (Figure 6.1). We also calculated the ratio of $\mathrm{p}$-tau/tau and found that both levels of $\mathrm{p}$-tau and the ratio of $\mathrm{p}$-tau/tau were increased compared to those from brains of mice without exposure to blast (Sham). The impact of blast to mouse brains was manifested as early as 3 hours, and remained after 24 hours, when significant reduction of tau and increase of p-tau were observed. Total tau level was decreased $\sim 20 \%$ compared to that of sham mouse brains, and the p-tau level was increased 1.2 fold, and these changes were found statistically significant. Thirty days after the blast, tau levels bounced back and remained at similar levels to those of sham mice. Similarly, p-tau dropped a statistically significant $20 \%$ after 30 days and then remained at similar levels to those of sham mice after 15 weeks (Figure 6.1). The ratios of $\mathrm{p}$-tau/tau reflected similar changes to the levels of $\mathrm{p}$-tau in mouse brains harvested at 30 days and 15 weeks after the blast.
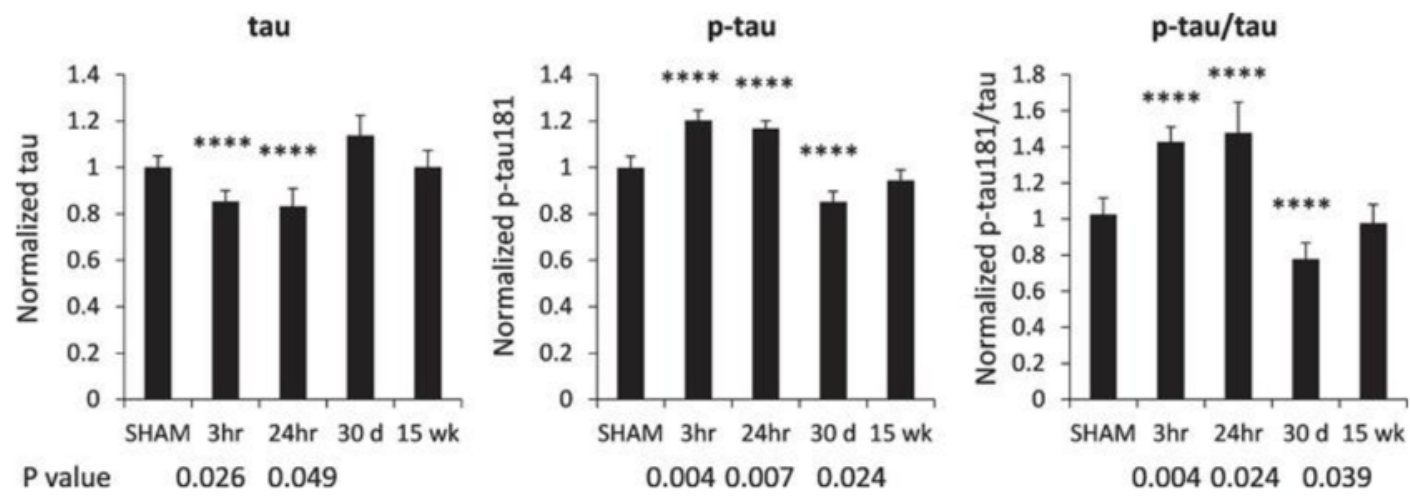

Figure 6.1 Quantification of total tau and phosphorylated tau from mouse brain at different time points after the blast. 
Brain lysates were subjected to ELISA using Meso Scale Discovery platform, and same samples were quantified for the levels of tau and $p$-tau. The ratio of $p$-tau to tau (ptau/tau) was calculated for each sample, and the levels of tau, p-tau, p-tau/tau in brains of control mice were normalized to 1 . Relative levels of these analytes in brains of mice harvested at 3 h, 24 h, 30 days, and 15 weeks after the blast were illustrated. The bar represents standard error of means (SEM). Asterisks represent statistically significant differences.

\subsubsection{Behavioral and pathological alteration in mice after the blast}

We performed explorative behavioral SNAP test on those animals exposed to 82-kPa low intensity blast (LIB). SNAP provides a sensitive, reliable, time-efficient and costeffective means of assessing neurological deficits in mice. Each individual test had a scoring range of 0-5 based on the assessment guidelines (Figure 6.2). Using the current settings and $350 \mathrm{~g}$ of high-energy explosive $\mathrm{C} 4$, SNAP assessments revealed significant difference in the blast group compared to sham controls at both 24 hrs and 7 days post injury (DPI) (Figure 6.2), indicating that neurological deficits affecting coordination, motor strength, proprioception, gait and mental status occurred after LIB exposure. 


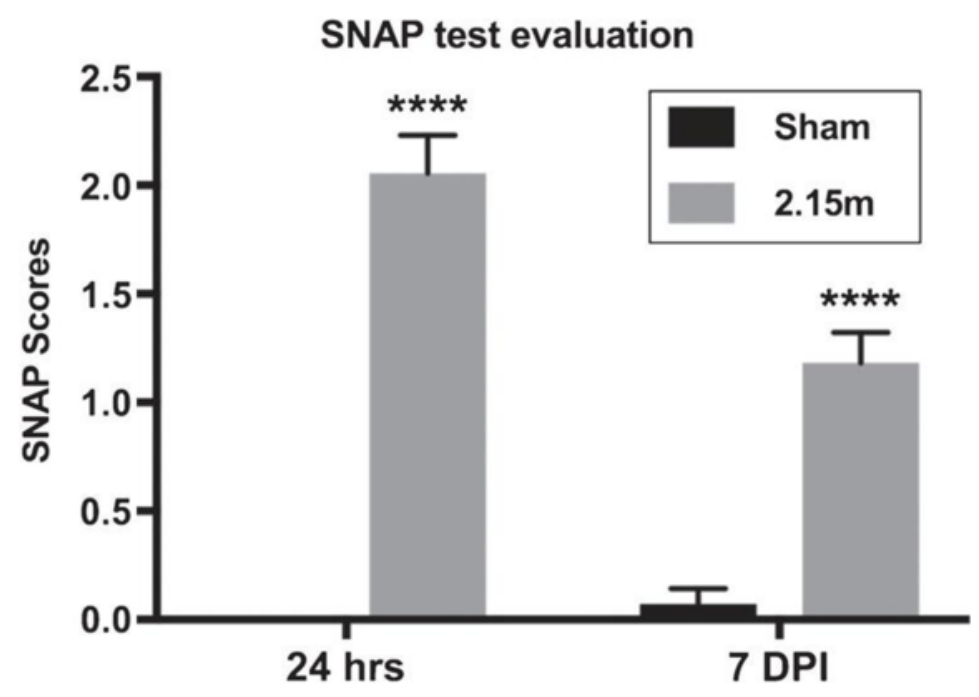

Figure 6.2 Neurological deficits after low intensity blast exposure assessed by SNAP test.

Neurological status was evaluated by SNAP tests at $24 \mathrm{~h}$ and 7 days post injury (DPI). All comparisons were performed between blast $(2.15 \mathrm{~m}$, distance between the animals and the blast) and sham group on the same DPI; $\mathrm{n}=5$ for sham, $\mathrm{n}=9$ at $24 \mathrm{~h}$ after blast, $\mathrm{n}=11$ at 7 DPI. Differences were considered significant at $\mathrm{p}<0.05$ for all analyses, with asterisks representing $\mathrm{p}<0.0001$.

Axonal injury in LIB-exposed animals occurred mainly at 7 DPI, as we previously described [25]. Degenerating axon-terminals and dendrites, with high affinity for silver (argyrophilia) were visualized on silver-stained tissue sections (Figure 6.3A-C). Densitometric analyses by NIH ImageJ were examined on various white matter regions rich for axons, including corpus callosum and entorhinal cortex, cerebral peduncle, and fornix. The silver stain intensities in blast group were significantly higher than in sham controls at 7 DPI for all subregions (Figure 6.3D-F). We have previously seen the 
abnormalities at 7 DPI appeared to return to comparable levels of sham controls at 30 DPI.
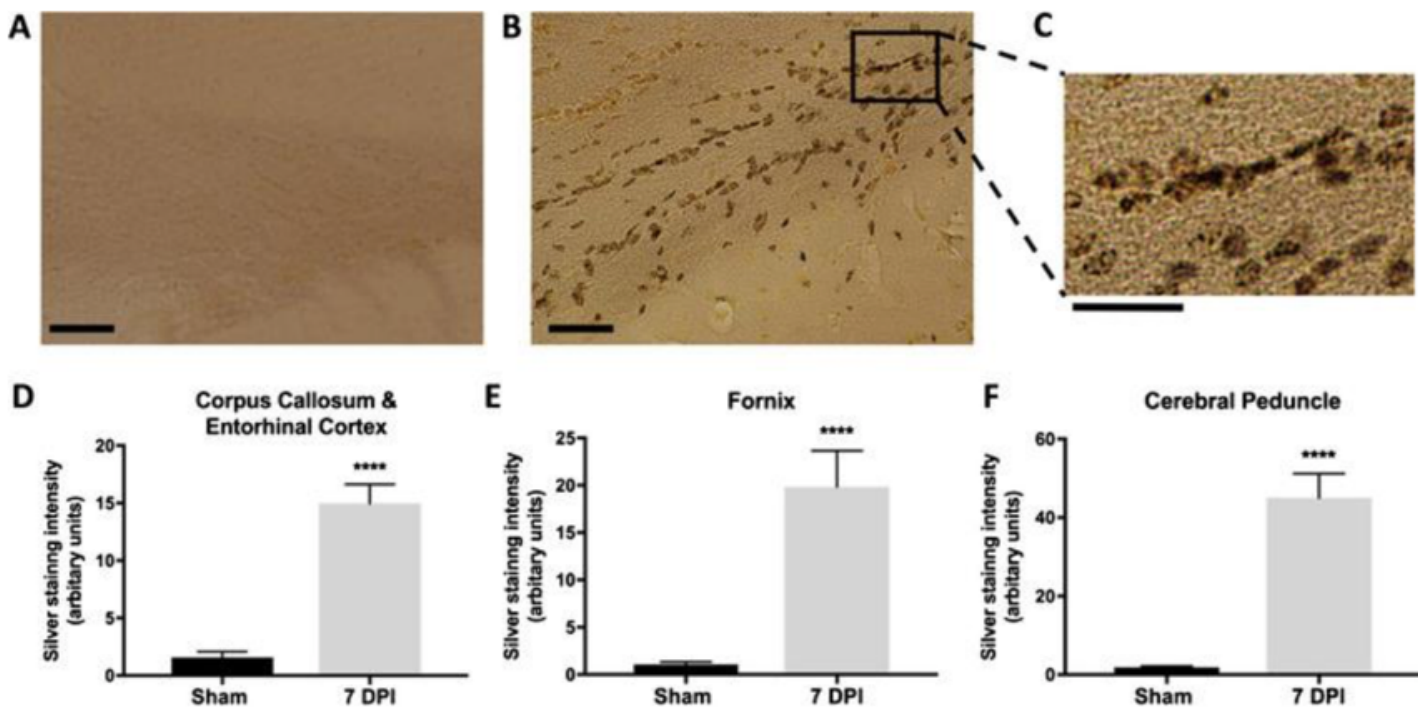

Figure 6.3 Axonal injury detected and quantified after LIB exposure by silver staining.

(A) Representative images from the sham group showed no axonal injury (scale bar $=50$ $\mu \mathrm{m})$. (B-C) Representative images from the blast group showed axonal injury at corpus callosum and entorhinal cortex (scale bar $=50 \mu \mathrm{m})$. Inset box represent the higher magnification of the indicated area (scale bar $=25 \mu \mathrm{m}$ ). (D-F) Intensity of silver staining in corpus callosum and entorhinal cortex, fornix, and cerebral peduncle regions in blast group at 7 DPI were significantly higher than that in the sham group. All comparisons were performed between blast and sham groups with t-test; $n=5$ for sham controls, and $n$ $=4$ for blast at 7 DPI. Differences were considered significant at $\mathrm{p}<0.05$ for all analyses, with asterisks representing $\mathrm{p}<0.0001$.

\subsubsection{Global protein alteration in brains exposed to blast}


To understand impact of blast to brain proteins that drive these phenotypes, analysis of both global proteomics and phospho proteomics were conducted using mouse brains collected at $3 \mathrm{hr}, 24 \mathrm{hr}, 30$ days and 15 weeks after the blast. We specifically searched for significant changes in proteins from mouse brains harvested at the time points ( 3 and 24 hrs) when we observed significant changes in tau and p-tau proteins. A total of 1692 proteins and 296 phosphoproteins were identified at global level and analyzed in this study. By comparing to the sham mouse brain, the fold of changes (ratio $\geq 1.2$ or $\leq 0.9$ ) of proteins observed in brains of mice at $3 \mathrm{hr}$ and $24 \mathrm{hr}$ after the blast were used for functional analysis by DAVID. At the global level, relative to the proteins identified from the sham controls, only a few proteins were down-regulated after the blast, including Hemoglobin subunit $\alpha$ (Hba) and Hemoglobin subunit $\beta-1$ (Hbb-b1). They are enriched in the cellular location of hemoglobin complex. Their primary molecular functions are oxygen transporter and oxygen/heme binding. On the other hand, 130 proteins were upregulated with the fold changes $\geq 1.2$ after the blast. DAVID functional analysis in three categories, including cellular components, biological process, and molecular function, of up-regulated proteins at $3 \mathrm{hr}$ after the blast were conducted (Figure 6.4A-C). The top two enriched cellular components (CC) are cytoplasm and neuronal cell body. Among cellular components, axon was also significantly enriched $(\mathrm{p}=1.2 \mathrm{E}-4)$, followed by other neuron related components, such as postsynaptic dendritic spine and microtubule (Figure 6.4A). Besides general biological processes (BP) such as transport and translation, most altered proteins were enriched in cell-cell adhesion and vesicle mediated transport. In addition, proteins enriched in axon guidance, axon extension and microtubule-based process were also illustrated (Figure 6.4B). The top enriched molecular functions (MF, Figure 6.4C) 
include cadherin binding/cell-cell adhesion, hydrolase activity, nucleotide binding,

microtubule binding, filamin binding and $\beta$-tubulin binding activities $(\mathrm{p} \leq 3.0 \mathrm{E}-2)$.

A

B

Retrograde vesicle-mediated transport, Golgi to ER Positive regulation of filopodium assembly

Translation

Actin filament organization

Learning

ER to Golgi vesicle-mediated transport

Axon guidance

Transport

Ketone body catabolic process

Positive regulation of axon extension

Microtubule-based process

Regulation of synaptic plasticity

Learning or memory

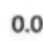

0.0

c

Cadherin binding involved in cell-cell adhesion

Phosphoprotein binding

Hydrolase activity

Nucleotide binding

Microtubule binding

Filamin binding

Calmodulin binding

GTPase activity

Aminopeptidase activity

Beta-tubulin binding

Structural constituent of ribosome

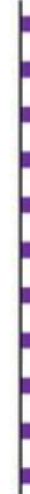

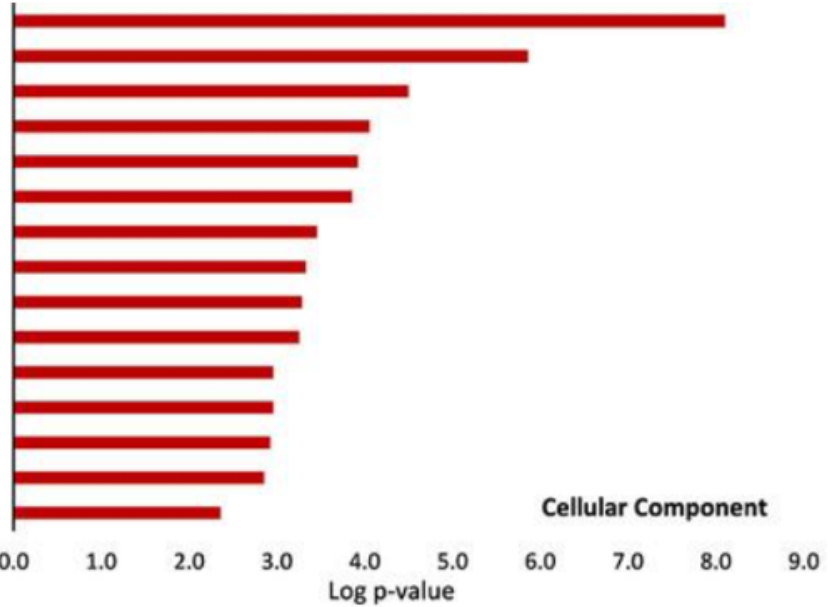

Log p-value

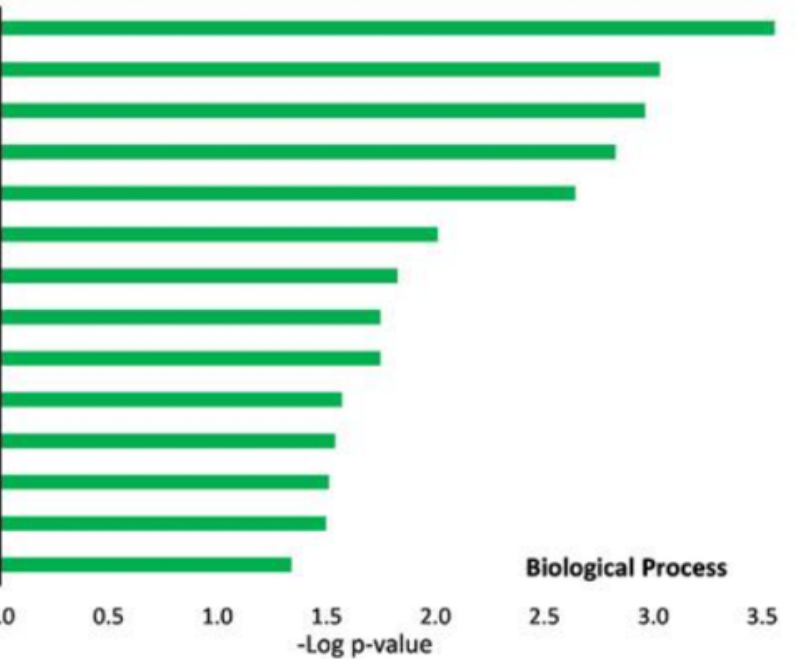

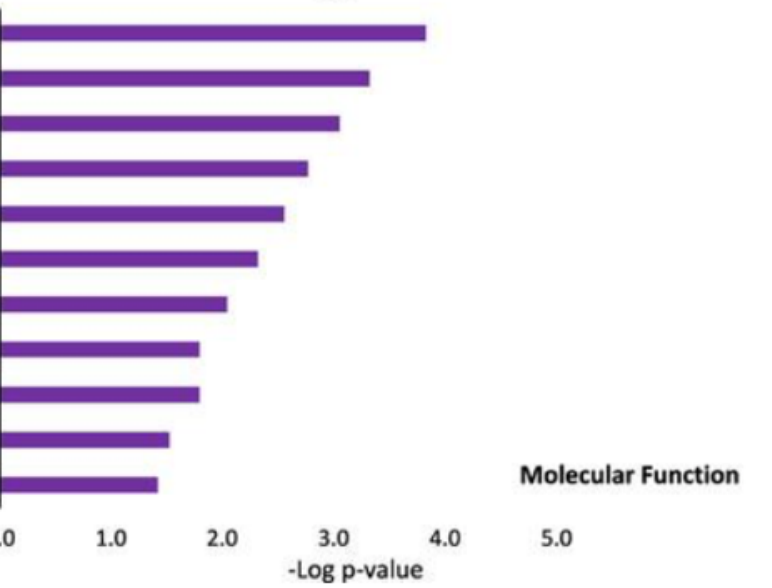

Figure 6.4 GO ontology analysis of altered proteins at global level. 
Proteins from mouse brains harvested at $3 \mathrm{hr}$ after the blast were analyzed, and each of the three categories, cellular components (A), biological process (B) and molecular functions (C) were listed. Proteins from blast-exposed animals were compared to those from control/sham animals, and - Log of p values was plotted.

We have examined proteins involved in each of the three top enriched categories $\mathrm{BP}, \mathrm{CC}$ and MF that are related to neuronal functions and listed up-regulated proteins from animals harvested at $3 \mathrm{hrs}$ post the blast. The biological processes included positive regulation of axon extension and axon-dendritic transport. The up regulated proteins highly enriched in postsynaptic density and axon included huntingtin (Htt), which may play a role in microtubule-mediated transport or vesicle function. Proteins involved in microtubule-based process include Tubulin $\alpha-1 \mathrm{~A}$ chain (Tub $\alpha 1 \mathrm{a})$ and Dynein light chain 1, cytoplasmic (Dynll1). GO enrichment analysis in each of the three categories (BP, CC, MF) revealed proteins related to those microtubule associated proteins, and network analysis of up- regulated proteins at 3hr were illustrated by STRING (Figure 6.5A). Proteins related to microtubule, such as Actr1b, Tuba1a, Tub $\beta 2 a$, Dunll1, are among the proteins with the extensive network connections. These proteins are also observed in mouse brains harvested at 24 hours (Figure 6.5B), and less in mouse brains harvested at 30 days (Figure 6.5C) and 15 weeks (Figure 6.5D) after the blast exposure. 


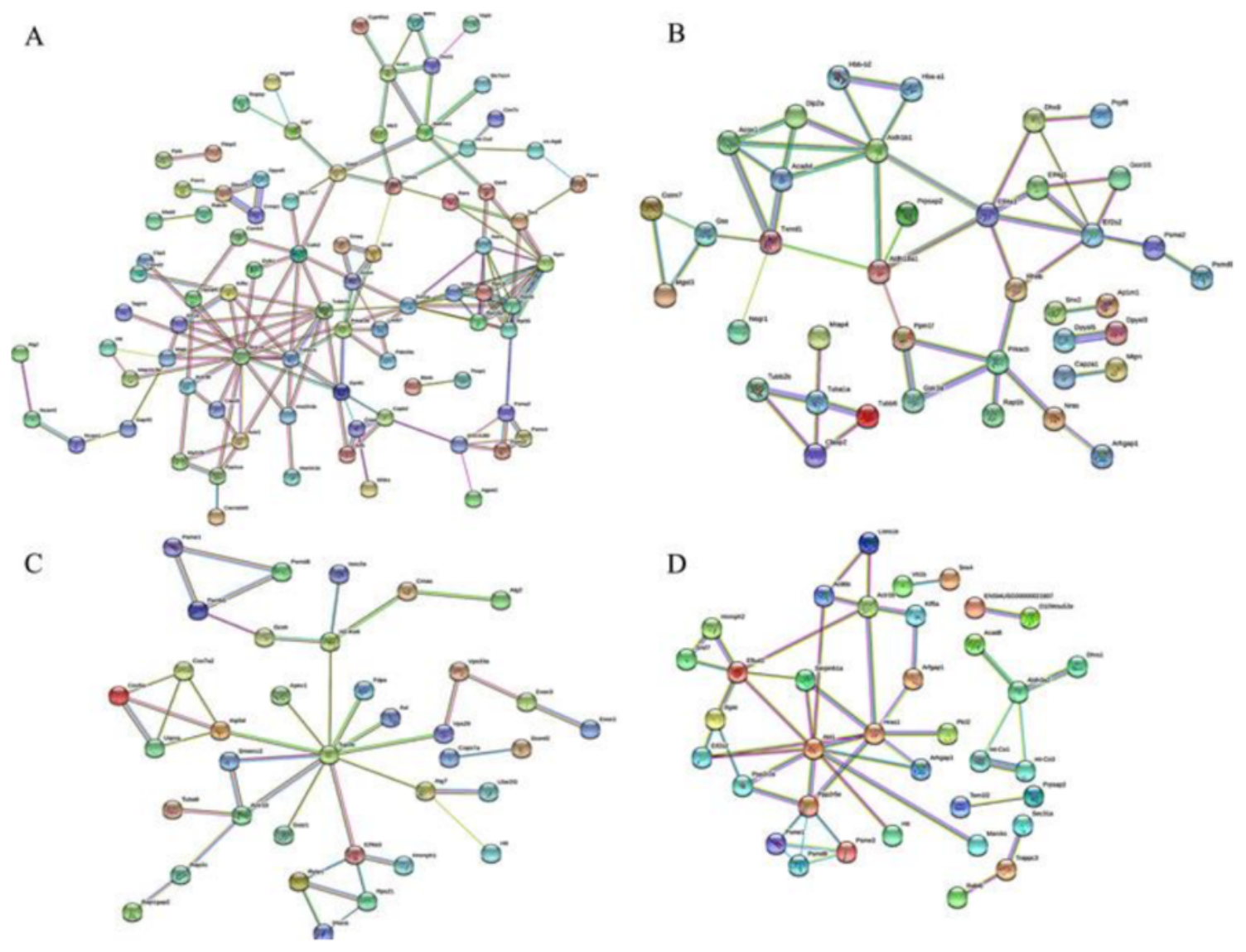

Figure 6.5 Network analysis of proteins was illustrated using the web-based tool STRING 10.0.

Proteins from mouse brains at $3 \mathrm{~h}(\mathrm{~A}), 24 \mathrm{~h}(\mathrm{~B}), 7$ days (C), and 15 weeks (D) after the blast were compared to those from the control group using LC-MS/MS.

KEGG pathway analysis of proteins shows that synthesis and degradation of ketone bodies $(p=4.1 E-3)$, dopaminergic synapse $(p=6.8 E-3)$, and ribosome $(p=4.0 E-2)$ were enriched. Those proteins involved in the dopaminergic synapse pathway include catechol-O-methyltransferase(Comt), guanine nucleotide binding protein (G protein) $\beta 4$ (Gnb4), guanine nucleotide binding protein, aq polypeptide (Gnaq), guanine nucleotide binding protein, astimulating, olfactory type (Gnal), kinesin family member 5C (Kif5c) 
and protein phosphatase 1, catalytic subunit, $\alpha$ isoform (Ppplca). How these proteins connect to each other and respond to blast-induced protein alteration remain to be investigated.

\subsubsection{Alteration of phospho proteomics in brains of mice exposed to blast}

We compared phospho proteins from mouse brains and captured 296 phosphorylated proteins for quantification. Among the list of altered phospho proteins at $3 \mathrm{hr}$ and $24 \mathrm{hr}$ after the blast, 29 phospho proteins were down-regulated and only one was up-regulated at $3 \mathrm{hr}$, whereas four were down regulated and 17 proteins were up regulated at $24 \mathrm{hr}$. Several clusters were enriched in these dysregulated proteins, such as synapse, cytoskeleton/actin binding, dendritic spine, microtubule, growth cone and neural projection. Based on DAVID analysis, the top enriched protein domains at $24 \mathrm{hr}$ include Stathmin family, C2 calcium-dependent membrane targeting protein and Synaptotagmin. The phospho proteins with largest increase include Add1, Camk2b, Syt1and Stmn1. Ap3b2, Sgip1, Basp1 and Rph3a were among those significantly down-regulated phosphoproteins.

GO ontology analysis of cellular components (CC) of dysregulated phosphoproteins revealed alteration in synaptic proteins (Figure 6.6A). At both time points, the down-regulated phosphoproteins are highly enriched in postsynaptic density, synapse, cell projection, and cytoskeleton $(\mathrm{p} \leq 4.40 \mathrm{E}-3)$. Those dysregulated phosphoproteins were enriched in brains harvested at $3 \mathrm{hr}$ and $24 \mathrm{hr}$ after the blast, respectively. At $3 \mathrm{hr}$ post blast, nearly $50 \%$ of the down-regulated phosphoproteins were enriched in cellular components related to synapse, postsynaptic density, myelin sheath 
and microtubule. At $24-\mathrm{hr}$ post blast, $33 \%$ of the proteins are enriched at synapse, followed by cytoskeleton (33\%), neuron projection (24\%), dendrite (24\%), and synaptic vesicle $(14 \%)$.
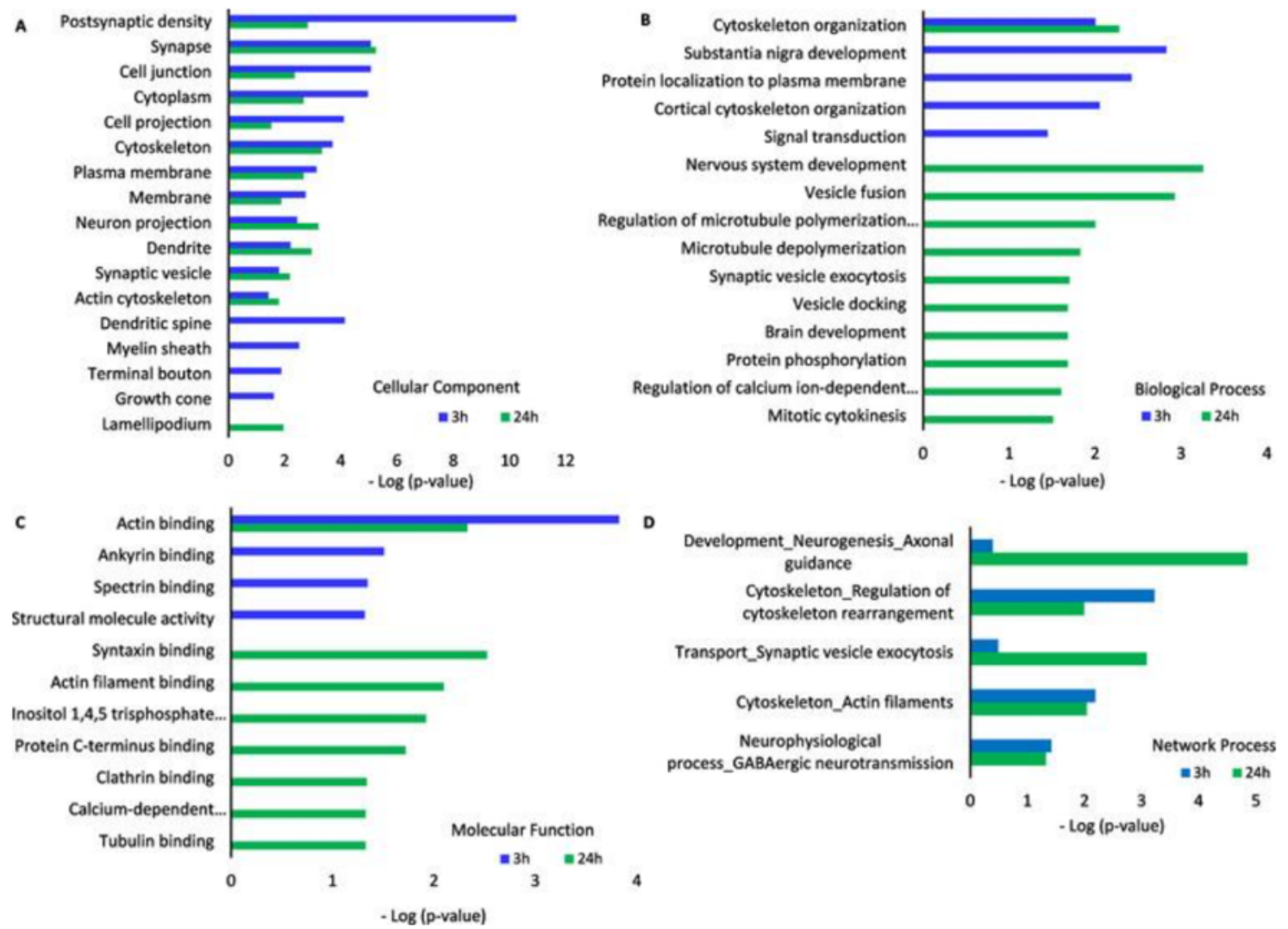

Figure 6.6 GO ontology analysis of altered brain phospho-proteomics at $3 \mathrm{~h}$ and 24

\section{h post impact.}

Cellular components (A), biological process (B), molecular functions (C), and network process (D) of phosphoproteins from mouse brains tissue at $3 \mathrm{~h}$ and $24 \mathrm{~h}$ after the blast were compared to those from control/sham group of animals.

The biological process (BP) of dysregulated phospho proteins was examined using GO ontology analysis (Figure 6.6B). The only common enriched biological process 
at both time points is cytoskeleton organization. In addition, substantia nigra degeneration and signal transduction at $3 \mathrm{hr}$ time point were also enriched. The top five enriched biological processes at $24 \mathrm{hr}$ are nervous system development, vesicle fusion and cytoskeleton organization, microtubule depolymerization and synaptic vesicle exocytosis. Molecular function (MF) of altered phosphoproteins from mouse brains at $3 \mathrm{hr}$ and $24 \mathrm{hr}$ after the blast were analyzed (Figure 6C). Actin binding is the only common MF among the dysregulated phospho-proteins at both time points. Other cytoskeleton related MF, such as ankyrin binding and spectrin binding were also enriched at $3 \mathrm{hr}$. At $24 \mathrm{hr}$, in additional to structural related MF, such as syntaxin binding and tubulin binding, calcium-dependent phospholipid is also enriched.

The top three GO process networks analysis of up- and down-regulated proteins were revealed using Metacore (Figure 6.6D). The networks related to cytoskeleton, such as Plectin 1, actin-binding Lim protein 1 (Ablim1) and neurofilament medium polypeptide (Nefm), were down regulated at both time points. Proteins related to the networks of synaptic contact and neurogenesis, such as Syt1, Stx7, Camk2b and Dlg2, were up regulated at 24hr (Figure 6.6D). Metacore analysis illustrated Cofilin as the top network for up-regulated phosphoproteins at $24 \mathrm{hr}$ after the blast.

When we analyzed mouse brains harvested at 30 days and 15 weeks after the blast exposure, we obtained similar results. Analysis of cellular components of phosphoproteins illustrated changes in synaptic proteins (Figure 6.7A). Those phosphoproteins were enriched in brains harvested at 30 days and 15 weeks after the blast, respectively. The biological process (BP) of dysregulated phospho proteins was examined using GO ontology analysis (Figure 6.7B). Similar to what we have observed 
in tissue collected at earlier time points, one enriched process at all four time points is cytoskeleton organization. Actin binding is the common molecular function among the phospho proteins at all time points (Figure 6.7C). The networks process of up- and downregulated proteins includes cytoskeleton and actin filament and development of neurogenesis and synaptogenesis (Figure 6.7D), similar to those identified at earlier time points (Figure 6.6D).
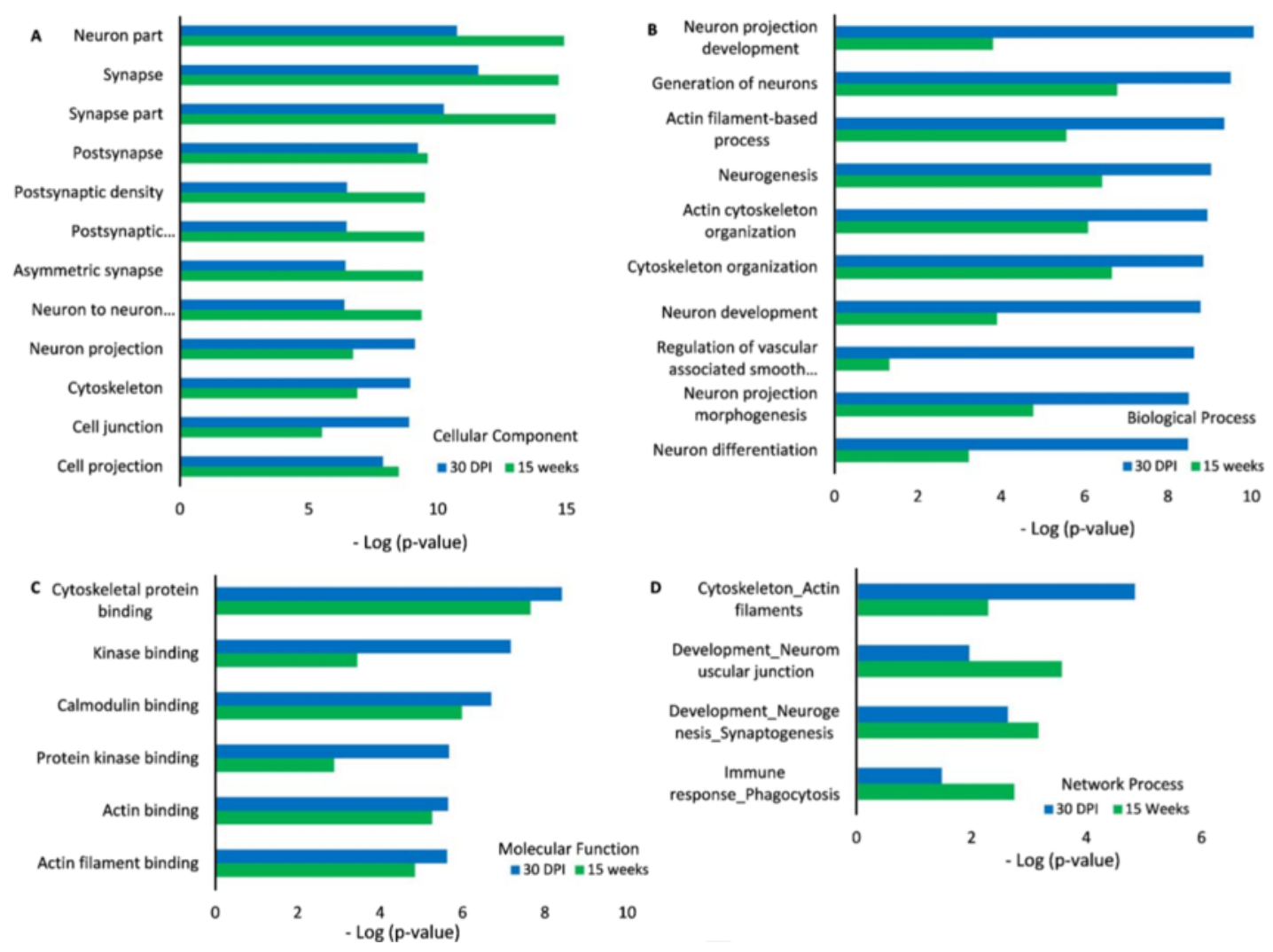

Figure 6.7 GO ontology analysis of altered brain phospho-proteomics at 30 days and

\section{5 weeks post impact.}

Cellular components (A), biological process (B), molecular functions (C), and network process (D) of phosphoproteins from mouse brains tissue at 30 days and 15 weeks after the blast were compared to those from control/sham group of animals. 
Network analysis revealed up-and down-regulated phosphoproteins at $3 \mathrm{hr}$ (Figure 6.8A), 24hr (Figure 6.8B), 30 days (Figure 6.8C), and 15 weeks (Figure 6.8D) using STRING. At the early time point $(3 \mathrm{hr})$, ATPase, $\mathrm{Ca}++$ transporting, plasma membrane 1 (ATP2b1), calcium/calmodulin-dependent protein kinase II, beta (Camk2b) and solute carrier family 25 (mitochondrial carrier, adenine nucleotide translocator), member 4 (Slc25a4) are all changed. KEGG pathway analysis shows that calcium signaling pathway is enriched among these altered proteins. At later time points, Cdk16 was a common protein identified by network analysis (Figure 6.8B-D).

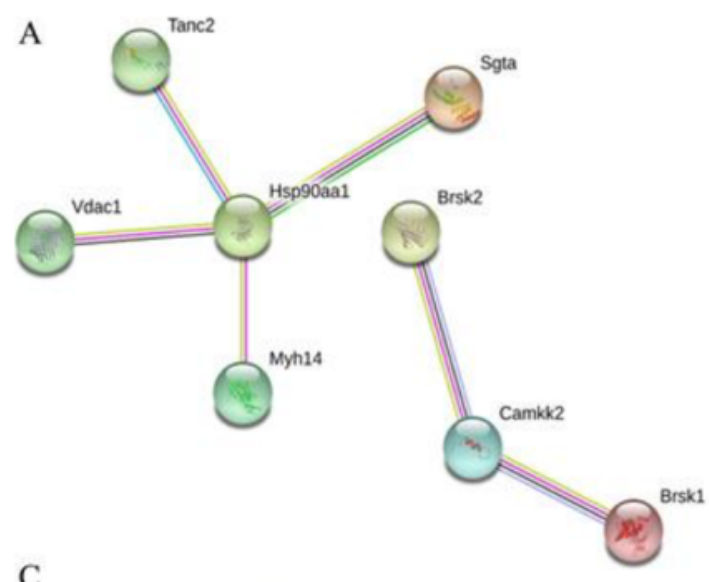

B

C
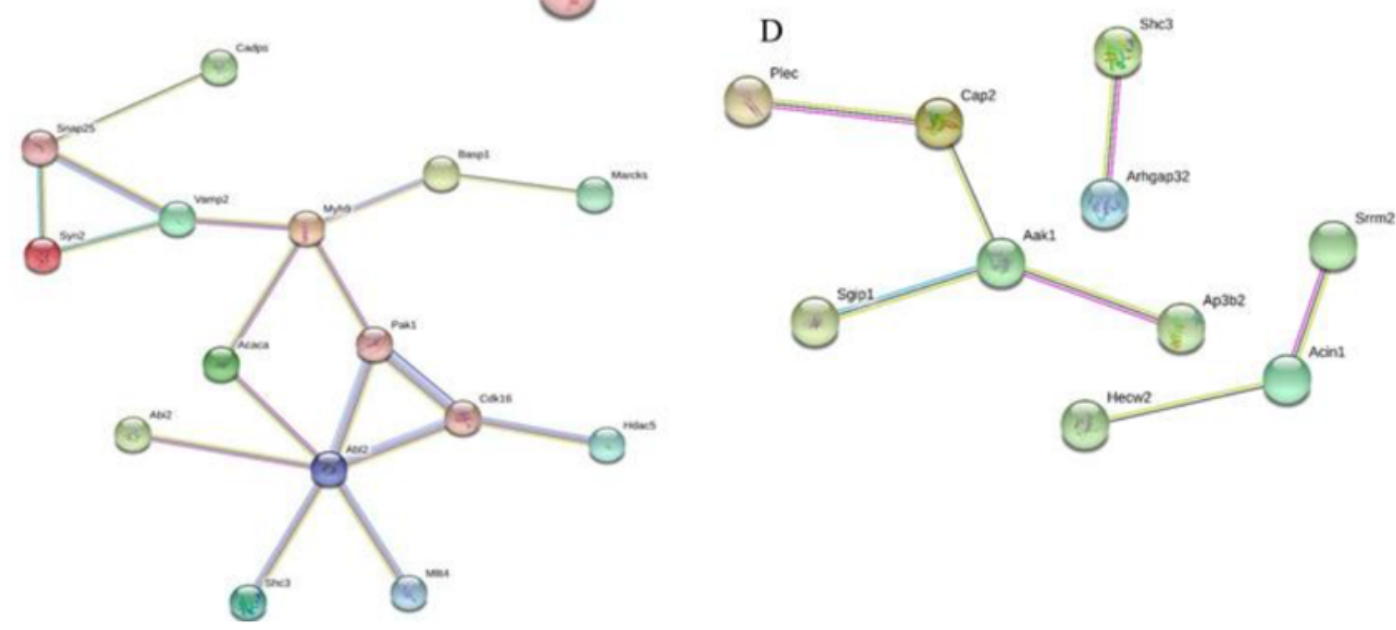

Figure 6.8 Network analysis of all captured phosphoproteins was illustrated using the web-based tool STRING. 
Proteins from mouse brains at $3 \mathrm{~h}$ (A), $24 \mathrm{~h}$ (B), 30 days (C), and 15 weeks (D) after the blast were compared to those from the control group using LC-MS/MS, and the enriched cellular components and altered proteins were analyzed by DAVID.

\subsection{DISCUSSION}

The significance of this study is highlighted by an urgent need to identify critical players involved in neuropathological development in people who suffer mTBI and to investigate how these factors contribute to neuronal damage and neurodegenerative diseases like AD. We have used an animal model exposed to open field blast to model mTBI [25, 26, 28]. This unique platform allows us to compare mice responses to brain injury at the molecular level of in-depth proteomics and biochemistry of brain tissue. Currently we lack mechanistic understanding of neuronal damages caused by mTBI and we do not have a target for therapeutic intervention for those suffering mTBI or in people with a history of mTBI. Based on the proteomic profiles of mouse brain exposed to blast, we are exploring novel targets and candidate proteins linked to axonal injury, microtubule cytoskeleton organization, and tau pathology.

\subsubsection{Proteomic analysis of the moue brain tissues revealed significant changes in protein expression representing axonal injury and microtubule cytoskeleton organization in response to injury}

This study utilized TMT which offers the ability to simultaneously detect ten samples under a single condition. DAVID analysis of altered proteins show that several functions 
significantly changed after the blast. Specifically, we found negative effect of blast on a number of cellular processes.

Diffuse axonal injury is a hallmark pathological consequence of non-penetrative mTBI [34]. In neuronal culture model, very mild stretch injury to a localized region of the cortical axon is able to trigger a degenerative response characterized by growth cone collapse and significant abnormal cytoskeleton rearrangement, resulting in the formation of smaller growth cones at the tips of axons and a significantly higher number of collapsed structures at both $24 \mathrm{~h}$ and $72 \mathrm{~h}$ post injury [34]. Axonal degeneration following stretch injury involves destabilization of the microtubule cytoskeleton. Microtubules are the stiffest structural components of axons and thus may be at risk of damage during mTBI. Loss of axonal microtubule was found after stretch injury and progressive disassembly of microtubules along the breakage points [35]. Stabilization of microtubule resulted in a significant reduction in the number of fragmented axons following injury [34]. Consistent with previous reports [36], our studies also revealed depolymerization of microtubules.

We identified several proteins, such as Map1B, Stmn1, Shc3, Sept7, Nefm, Ap3b2, Mapt. They play important roles in maintaining microtubule stability and axonal integrity. Map1B belongs to a group of microtubule-associated proteins that support microtubule-stabilization, tubulin assembly/disassembly, and physical interactions between microtubules and components of the cytoskeleton. Map1B and its phosphorylated isoform are present in growing axons and concentrated in distal end near the growth cone. Map1B plays a crucial role in the stability of the cytoskeleton, and its function is modulated by the state of phosphorylation. Immuno-histochemical staining 
showed that phosphorylated Map1B was abundant in developing axons, suggesting its essential role in axonal elongation [37]. Map1B is up-regulated after ischemia in aged rats [38] but down-regulated in optic nerve after mTBI [36]. In this study, down regulation of phosphorylated Map1B was indicative of axonal injury and cytoskeletal damage following TBI. It is possible that the structural reorganization at the cellular level and shifts in cytoskeletal dynamic associate with injury. This observation is consistent with our previous finding of down-regulated phosphorylated Map1B from the inferior prefrontal cortex region of post-mortem brain tissues from AD patients [39], clearly linking the TBI-induced cytoskeletal damage to pathological changes in $\mathrm{AD}$ brain. Stathmin (Stmn) plays an essential role in the maintenance of axonal integrity. It is involved in the regulation of the microtubule filament system by destabilizing microtubules, and phosphorylation of Stmn regulates microtubule disassembly. In our study, phosphorylated Stmn1 and Stmn3 were found up-regulated at $24 \mathrm{hr}$ after the blast, and the level of Stmn1 was found 2 fold of that of the control group at 15 weeks after the blast. A recent study demonstrated that Stmn-dependent changes in microtubule stability are crucial for synaptic function and memory formation [40]. Stmn acts on microtubule disruption and/or tubulin sequestration, and elevation of Stmn1 after blasts was found in rat model of mTBI [41]. High Stmn expression induced microtubule structures disruption and enhanced tau hyperphosphorylation [42]. Similarly, brain injury causes reduced polymerization of Gephyrin and subsequent synapse destabilization [43], and progressive disassembly and loss of axonal microtubule along the breakage points [35].

A number of proteins altered in our mice exposed to open field blast were also reported from previous proteomic analysis of optic nerves from mice suffering repetitive 
mTBI (rmTBI) [36]. Various negative effects of rmTBI on cellular processes were reported, including depolymerization of microtubules, disassembly of filaments and loss of neurons, evidenced by a reduction of several proteins, including neurofilaments, tubulin (Tubb2A, Tuba4A), and microtubule-associated proteins (Map1A, Map1B) [36]. In our study, phosphorylated neurofilament medium polypeptide (Nefm) was down regulated. Nefm belongs to the group of neurofilament triplet proteins, the major cytoskeletal components of neurons in the CNS. They interact with microtubules and microfilaments to form and maintain the axonal structural integrity and neuronal shape, and also participate to the axonal mechanism of transport. Significant decrease in neurofilament levels in optic nerve of rat retina after 21 days mTBI was reported [36]. Furthermore, increased level of Nefm was observed in CSF and serum from patients with a history of mTBI, but the effect could come from peripheral nerve injury [44].

We also observed several altered dynein proteins in addition to those proteins related to microtubule and tubulin binding. Axonal transport is related to microtubule stability and essential for transducing extracellular signals and recycling misfolded proteins from the nerve terminals to cell soma, thus avoiding the build-up of toxic aggregates. Axonal transport requires the cytoskeletal microtubule scaffold to serve as tracks, and the motor proteins kinesin and dynein that exert mechanical force to move cargoes and retrogradely, respectively.

In this study, we found changes in a large proportion of proteins located in dendritic spines, those highly dynamic membranous protrusions on post-synaptic dendrites. Structural changes in dendritic spines form the basis for learning and memory formation, making them central hubs for the processing and storage of information in the 
brain [45]. Study in Map1B-deficient mice showed that Map1B was required for dendritic spine development and synaptic maturation [46]. Study of mouse hippocampal neuron cultures showed inhibition of microtubule dynamics alters dendritic spine morphology through the actin cytoskeleton, suggesting that the microtubule and actin cytoskeleton act together in dendritic spines to regulate synaptic plasticity [47]. We have observed changes in several proteins with actin binding activities. Three phosphorylated actin binding proteins, Cofilin, Add1 and Ccdc88a, were found up-regulated $24 \mathrm{hr}$ after the blast in our study. Cofilin is a major component of intranuclear and cytoplasmic actin rods, and Ccdc88a is required for formation of actin stress fibers and lamellipodia.

Consistent with previous study [36], phospho myelin basic protein (Mbp) was found reduced in mice exposed to blast in our study. Mbp is a major constituent of CNS myelin and mature oligodendrocytes, and the decrease in Mbp levels could be a direct reflection of an ongoing demyelination. We observed continuous decrease of phospho Mbp at 15 weeks, suggesting that demyelination was a long lasting outcome of TBI. In Schwann cells responding to two hours of sustained shear stress, Mbp appeared to be down regulated, suggesting that shear stress alone is sufficient to induce pathological changes [48]. In rats, extensive degradation of Mbp isoforms was observed after TBI [49], suggesting that TBI-mediated axonal injury causes secondary structural damage to the adjacent myelin membrane and instigate Mbp degradation. This could initiate myelin sheath instability and demyelination, which might further promote axonal vulnerability [49].

\subsubsection{Up-regulated calcium signaling after TBI}


In our study, several phosphorylated proteins involved in calcium signaling pathway were up-regulated. There is strong evidence in support of the concept that abnormal neurotransmission and $\mathrm{Ca}^{2+}$-mediated signal transduction pathways could be responsible for neuronal dysfunction following trauma. Calcium is crucial for several aspects of plasticity at glutamatergic synapses. Changes in the levels of intracellular $\mathrm{Ca}^{2+}$ or alterations of $\mathrm{Ca}^{2+}$ signaling could contribute to a variety of pathologies. One of the major causes of secondary injury following TBI is related to an excess influx of $\mathrm{Ca}^{2+}$ into cells in the brain [50].

Calcium/calmodulin-dependent protein kinase type II subunit beta $(\mathrm{Camk} 2 \mathrm{~b})$ is essential for the integrity of the actin cytoskeleton and for cell migration.Camk $2 b$ is expressed in motor neurons during axon outgrowth and is part of slow axonal transport [51]. Consistent with previous findings [52, 53],we found increased phosphorylation of CamkIIb at 24hr after the TBI. Atp2b1, which mediates calcium efflux, was up regulated in our blast-induced mouse brains. Slc25a4, which catalyzes the exchange of ADP and ATP across the mitochondrial inner membrane, was also up regulated. Since the response to CNS injury includes an apparent increase in energy mobilization capacity, elevated intracellular $\mathrm{Ca}^{2+}$ after TBI may be related to reduced ATP levels, as previously reported [50].Another key protein, phosphorylated Synaptotagmin I (Syt1), was up-regulated at $24 \mathrm{hr}$ after the blast. Synaptotagmins are integral membrane proteins of synaptic vesicles thought to serve as $\mathrm{Ca}^{2+}$ sensors in the process of vesicular trafficking and exocytosis. Calcium binding Syt-1 participates in triggering neurotransmitter release at the synapse [53]. 


\subsubsection{Increases of p-tau, behavioral and pathological changes in mice exposed to blast link mTBI to AD}

Our study revealed pathways related to p-tau (Figure 6.1), which is the component of neurofibrillary tangles found in brains of AD patients. In addition, our exploratory SNAP tests revealed significant difference behavioral outcomes from the blast group compared to sham controls (Figure 6.2). Degenerating axon-terminals and dendrites were visualized on silver-stained tissue sections from mouse brains exposed to blast (Figure 6.3), supporting a contributing role of mTBI toward neurodegenerative process commonly found in brains of $\mathrm{AD}$ patients.

Tau as axonal injury marker has been reported in previous studies following mTBI $[54,55]$. Traumatic brain injury can lead to diffuse traumatic axonal injury and blood-brain barrier disruption. Shearing of axons results in the disruption of tau binding to tubulin [56]. Subsequent hyperphosphorylation of tau leads to formation of tau oligomers in the neuronal soma. When tau becomes hyperphosphorylated, it binds to other normal tau proteins, which leads to aggregation. Accumulation of insoluble tau within neurons contributes to the development of tau oligomers [56]. Tau oligomers are granular intracellular buildups of mutated tau, which precede the development of NFTs. Tau hyperphosphorylation is common after mTBI and other brain injuries [57]. Previous study in patients with mTBI showed the level of phosphorylated tau was significantly higher and correlated with the Glasgow Coma Scale (GCS) score [58]. We found a similar increase of p-tau in brains of mice collected at 3 and 24 hours after open field blast exposure, which is consistent with previous findings in humans and animals [5860]. While increase in tau immunostaining in brain tissue was not observed in mini-pig 
from 2 weeks to 8 months after blast-induced TBI [61], our ELISA-based quantitation of tau and phospho-tau in mice exposed to blast illustrate a significant difference at 3 and 24 hours. Levels of tau and p-tau were significantly changed within one day after the blast and returned to pre-blast stage at 15 weeks after blast (Figure 6.1). This is consistent with previous findings in mini-pigs [61] and mice [63]. In transgenic mice expressing human tau (hTau), only transient increases in p-tau was observed after single or repetitive mild TBI, and no tau pathology was found at one year after TBI [63].

Tau-related pathological change is a common feature linking mTBI to AD. Whether mTBI-induced phospho-tau increase triggers AD pathogenesis needs to be investigated. Elucidation of tau-related molecular changes in people with a history of mTBI will open a new window to determine whether personnel who have suffered mTBI have asymptomatic $\mathrm{AD}$. This is essential for designing future preventive measures for AD. Future effort to suppress p-tau-related neuropathological changes will potentially reduce the burden on affected individuals, especially those vulnerable to developing AD. 


\subsection{REFERENCES}

[1] H.T. Tran, F.M. LaFerla, D.M. Holtzman, D.L. Brody, Controlled cortical impact traumatic brain injury in 3xTg-AD mice causes acute intra-axonal amyloid-beta accumulation and independently accelerates the development of tau abnormalities, J Neurosci 31(26) (2011) 9513-25.

[2] S.T. DeKosky, E.E. Abrahamson, J.R. Ciallella, W.R. Paljug, S.R. Wisniewski, R.S. Clark, M.D. Ikonomovic, Association of increased cortical soluble abeta42 levels with diffuse plaques after severe brain injury in humans, Arch Neurol 64(4) (2007) 541-4. [3] M.D. Ikonomovic, K. Uryu, E.E. Abrahamson, J.R. Ciallella, J.Q. Trojanowski, V.M. Lee, R.S. Clark, D.W. Marion, S.R. Wisniewski, S.T. DeKosky, Alzheimer's pathology in human temporal cortex surgically excised after severe brain injury, Exp Neurol 190(1) (2004) 192-203.

[4] J.E. Bailes, A.L. Petraglia, B.I. Omalu, E. Nauman, T. Talavage, Role of subconcussion in repetitive mild traumatic brain injury, J Neurosurg 119(5) (2013) 123545.

[5] V.E. Johnson, W. Stewart, D.H. Smith, Widespread tau and amyloid-Beta pathology many years after a single traumatic brain injury in humans, Brain Pathol 22(2) (2012) $142-9$.

[6] B.E. Gavett, R.C. Cantu, M. Shenton, A.P. Lin, C.J. Nowinski, A.C. McKee, R.A. Stern, Clinical appraisal of chronic traumatic encephalopathy: current perspectives and future directions, Curr Opin Neurol 24(6) (2011) 525-31.

[7] L.E. Goldstein, A.M. Fisher, C.A. Tagge, X.L. Zhang, L. Velisek, J.A. Sullivan, C. Upreti, J.M. Kracht, M. Ericsson, M.W. Wojnarowicz, C.J. Goletiani, G.M. 
Maglakelidze, N. Casey, J.A. Moncaster, O. Minaeva, R.D. Moir, C.J. Nowinski, R.A. Stern, R.C. Cantu, J. Geiling, J.K. Blusztajn, B.L. Wolozin, T. Ikezu, T.D. Stein, A.E. Budson, N.W. Kowall, D. Chargin, A. Sharon, S. Saman, G.F. Hall, W.C. Moss, R.O. Cleveland, R.E. Tanzi, P.K. Stanton, A.C. McKee, Chronic traumatic encephalopathy in blast-exposed military veterans and a blast neurotrauma mouse model, Sci Transl Med 4(134) (2012) 134ra60.

[8] H. Terrio, L.A. Brenner, B.J. Ivins, J.M. Cho, K. Helmick, K. Schwab, K. Scally, R. Bretthauer, D. Warden, Traumatic brain injury screening: preliminary findings in a US Army Brigade Combat Team, J Head Trauma Rehabil 24(1) (2009) 14-23.

[9] C.L. Mac Donald, O.R. Adam, A.M. Johnson, E.C. Nelson, N.J. Werner, D.J. Rivet, D.L. Brody, Acute post-traumatic stress symptoms and age predict outcome in military blast concussion, Brain 138(Pt 5) (2015) 1314-26.

[10] C.L. Mac Donald, J. Barber, M. Jordan, A.M. Johnson, S. Dikmen, J.R. Fann, N. Temkin, Early Clinical Predictors of 5-Year Outcome After Concussive Blast Traumatic Brain Injury, JAMA Neurol 74(7) (2017) 821-829.

[11] R.D. Vanderploeg, H.G. Belanger, R.D. Horner, A.M. Spehar, G. Powell-Cope, S.L. Luther, S.G. Scott, Health outcomes associated with military deployment: mild traumatic brain injury, blast, trauma, and combat associations in the Florida National Guard, Arch Phys Med Rehabil 93(11) (2012) 1887-95.

[12] D.K. Menon, A.I. Maas, Traumatic brain injury in 2014. Progress, failures and new approaches for TBI research, Nat Rev Neurol 11(2) (2015) 71-2.

[13] M. Hutton, Molecular genetics of chromosome 17 tauopathies, Ann N Y Acad Sci $920(2000) 63-73$. 
[14] J. Lewis, E. McGowan, J. Rockwood, H. Melrose, P. Nacharaju, M. Van

Slegtenhorst, K. Gwinn-Hardy, M. Paul Murphy, M. Baker, X. Yu, K. Duff, J. Hardy, A. Corral, W.L. Lin, S.H. Yen, D.W. Dickson, P. Davies, M. Hutton, Neurofibrillary tangles, amyotrophy and progressive motor disturbance in mice expressing mutant (P301L) tau protein, Nat Genet 25(4) (2000) 402-5.

[15] J. Gotz, F. Chen, J. van Dorpe, R.M. Nitsch, Formation of neurofibrillary tangles in P3011 tau transgenic mice induced by Abeta 42 fibrils, Science 293(5534) (2001) 1491-5. [16] K. Santacruz, J. Lewis, T. Spires, J. Paulson, L. Kotilinek, M. Ingelsson, A. Guimaraes, M. DeTure, M. Ramsden, E. McGowan, C. Forster, M. Yue, J. Orne, C. Janus, A. Mariash, M. Kuskowski, B. Hyman, M. Hutton, K.H. Ashe, Tau suppression in a neurodegenerative mouse model improves memory function, Science 309(5733) (2005) $476-81$.

[17] A.M. Fagan, C.M. Roe, C. Xiong, M.A. Mintun, J.C. Morris, D.M. Holtzman, Cerebrospinal fluid tau/beta-amyloid(42) ratio as a prediction of cognitive decline in nondemented older adults, Arch Neurol 64(3) (2007) 343-9.

[18] O. Hansson, H. Zetterberg, P. Buchhave, E. Londos, K. Blennow, L. Minthon, Association between CSF biomarkers and incipient Alzheimer's disease in patients with mild cognitive impairment: a follow-up study, Lancet Neurol 5(3) (2006) 228-34.

[19] L.M. Shaw, H. Vanderstichele, M. Knapik-Czajka, M. Figurski, E. Coart, K. Blennow, H. Soares, A.J. Simon, P. Lewczuk, R.A. Dean, E. Siemers, W. Potter, V.M. Lee, J.Q. Trojanowski, Qualification of the analytical and clinical performance of CSF biomarker analyses in ADNI, Acta Neuropathol 121(5) (2011) 597-609. 
[20] M. Bacioglu, L.F. Maia, O. Preische, J. Schelle, A. Apel, S.A. Kaeser, M.

Schweighauser, T. Eninger, M. Lambert, A. Pilotto, D.R. Shimshek, U. Neumann, P.J.

Kahle, M. Staufenbiel, M. Neumann, W. Maetzler, J. Kuhle, M. Jucker, Neurofilament

Light Chain in Blood and CSF as Marker of Disease Progression in Mouse Models and in Neurodegenerative Diseases, Neuron 91(1) (2016) 56-66.

[21] G.A. Sarkis, M.D. Mangaonkar, A. Moghieb, B. Lelling, M. Guertin, H. Yadikar, Z. Yang, F. Kobeissy, K.K. Wang, The Application of Proteomics to Traumatic Brain and Spinal Cord Injuries, Curr Neurol Neurosci Rep 17(3) (2017) 23.

[22] A.M. Boutte, C. Yao, F. Kobeissy, X.C. May Lu, Z. Zhang, K.K. Wang, K. Schmid, F.C. Tortella, J.R. Dave, Proteomic analysis and brain-specific systems biology in a rodent model of penetrating ballistic-like brain injury, Electrophoresis 33(24) (2012) 3693-704.

[23] H. Song, S. Fang, J. Gao, J. Wang, Z. Cao, Z. Guo, Q. Huang, Y. Qu, H. Zhou, J. Yu, Quantitative Proteomic Study Reveals Up-Regulation of cAMP Signaling PathwayRelated Proteins in Mild Traumatic Brain Injury, J Proteome Res 17(2) (2018) 858-869. [24] G.K. Zupanc, Proteomics of traumatic brain injury and regeneration, Proteomics Clin Appl 1(11) (2007) 1362-72.

[25] H. Song, L.M. Konan, J. Cui, C.E. Johnson, M. Langenderfer, D. Grant, T. Ndam, A. Simonyi, T. White, U. Demirci, D.R. Mott, D. Schwer, G.K. Hubler, I. Cernak, R.G. DePalma, Z. Gu, Ultrastructural brain abnormalities and associated behavioral changes in mice after low-intensity blast exposure, Behav Brain Res 347 (2018) 148-157. 
[26] H. Song, L. Konan, J. Cui, C. Johnson, G. Hubler, R. DePalma, Z. Gu, Nanometer ultrastructural brain damage following low intensity primary blast wave exposure, Neural Regeneration Research 13(9) (2018) 1516-1519.

[27] S.B. Shelton, D.B. Pettigrew, A.D. Hermann, W. Zhou, P.M. Sullivan, K.A.

Crutcher, K.I. Strauss, A simple, efficient tool for assessment of mice after unilateral cortex injury, J Neurosci Methods 168(2) (2008) 431-42.

[28] H. Song, J. Cui, A. Simonyi, C.E. Johnson, G.K. Hubler, R.G. DePalma, Z. Gu, Linking blast physics to biological outcomes in mild traumatic brain injury: Narrative review and preliminary report of an open-field blast model, Behav Brain Res 340 (2018) 147-158.

[29] M.C. Johnson, H. Song, J. Cui, V.V. Mossine, Z. Gu, C.M. Greenlief, Development of a Method and Validation for the Quantitation of FruArg in Mice Plasma and Brain Tissue Using UPLC-MS/MS, ACS Omega 1(4) (2016) 663-668.

[30] R.E. Bennett, D.L. Brody, Acute reduction of microglia does not alter axonal injury in a mouse model of repetitive concussive traumatic brain injury, J Neurotrauma 31(19) (2014) 1647-63.

[31] H.K. Lee, C. Sanchez, M. Chen, P. Morin, J. Wells, E. Hanlon, W. Xia, Three Dimensional Human Neuro-Spheroid Model of Alzheimer's Disease Based on Differentiated Induced Pluripotent Stem Cells., PloS One (2016) In press.

[32] D.W. Huang, B.T. Sherman, R.A. Lempicki, Systematic and integrative analysis of large gene lists using DAVID bioinformatics resources, Nat Protoc 4(1) (2009) 44-57. [33] D.W. Huang, B.T. Sherman, Q. Tan, J.R. Collins, W.G. Alvord, J. Roayaei, R. Stephens, M.W. Baseler, H.C. Lane, R.A. Lempicki, The DAVID Gene Functional 
Classification Tool: a novel biological module-centric algorithm to functionally analyze large gene lists, Genome Biol 8(9) (2007) R183.

[34] Y.C. Yap, A.E. King, R.M. Guijt, T. Jiang, C.A. Blizzard, M.C. Breadmore, T.C.

Dickson, Mild and repetitive very mild axonal stretch injury triggers cystoskeletal mislocalization and growth cone collapse, PLoS One 12(5) (2017) e0176997.

[35] M.D. Tang-Schomer, A.R. Patel, P.W. Baas, D.H. Smith, Mechanical breaking of microtubules in axons during dynamic stretch injury underlies delayed elasticity, microtubule disassembly, and axon degeneration, Faseb J 24(5) (2010) 1401-10. [36] R. Tzekov, C. Dawson, M. Orlando, B. Mouzon, J. Reed, J. Evans, G. Crynen, M. Mullan, F. Crawford, Sub-Chronic Neuropathological and Biochemical Changes in Mouse Visual System after Repetitive Mild Traumatic Brain Injury, PLoS One 11(4) (2016) e0153608.

[37] R. Sato-Yoshitake, Y. Shiomura, H. Miyasaka, N. Hirokawa, Microtubuleassociated protein 1B: molecular structure, localization, and phosphorylation-dependent expression in developing neurons, Neuron 3(2) (1989) 229-38.

[38] D.C. Dieterich, N. Trivedi, R. Engelmann, E.D. Gundelfinger, P.R. Gordon-Weeks, M.R. Kreutz, Partial regeneration and long-term survival of rat retinal ganglion cells after optic nerve crush is accompanied by altered expression, phosphorylation and distribution of cytoskeletal proteins, Eur J Neurosci 15(9) (2002) 1433-43.

[39] M. Chen, H.K. Lee, L. Moo, E. Hanlon, T. Stein, W. Xia, Common proteomic profiles of induced pluripotent stem cell-derived three-dimensional neurons and brain tissue from Alzheimer patients, J Proteomics 182 (2018) 21-33. 
[40] S. Uchida, G. Martel, A. Pavlowsky, S. Takizawa, C. Hevi, Y. Watanabe, E.R. Kandel, J.M. Alarcon, G.P. Shumyatsky, Learning-induced and stathmin-dependent changes in microtubule stability are critical for memory and disrupted in ageing, Nat Commun 5(4389) (2014) 4389.

[41] G.A. Elder, N.P. Dorr, R. De Gasperi, M.A. Gama Sosa, M.C. Shaughness, E. Maudlin-Jeronimo, A.A. Hall, R.M. McCarron, S.T. Ahlers, Blast exposure induces posttraumatic stress disorder-related traits in a rat model of mild traumatic brain injury, $\mathrm{J}$ Neurotrauma 29(16) (2012) 2564-75.

[42] T. Miyasaka, S. Sato, Y. Tatebayashi, A. Takashima, Microtubule destruction induces tau liberation and its subsequent phosphorylation, FEBS Lett 584(14) (2010) 3227-32.

[43] D.P. Bonislawski, E.P. Schwarzbach, A.S. Cohen, Brain injury impairs dentate gyrus inhibitory efficacy, Neurobiol Dis 25(1) (2007) 163-9.

[44] E. Martinez-Morillo, C. Childs, B.P. Garcia, F.V. Alvarez Menendez, A.D. Romaschin, G. Cervellin, G. Lippi, E.P. Diamandis, Neurofilament medium polypeptide (NFM) protein concentration is increased in CSF and serum samples from patients with brain injury, Clin Chem Lab Med 53(10) (2015) 1575-84.

[45] J.N. Bourne, K.M. Harris, Balancing structure and function at hippocampal dendritic spines, Annu Rev Neurosci 31 (2008) 47-67.

[46] E. Tortosa, C. Montenegro-Venegas, M. Benoist, S. Hartel, C. Gonzalez-Billault, J.A. Esteban, J. Avila, Microtubule-associated protein 1B (MAP1B) is required for dendritic spine development and synaptic maturation, J Biol Chem 286(47) (2011) 40638-48. 
[47] E.B. Merriam, M. Millette, D.C. Lumbard, W. Saengsawang, T. Fothergill, X. Hu, L. Ferhat, E.W. Dent, Synaptic regulation of microtubule dynamics in dendritic spines by calcium, F-actin, and drebrin, J Neurosci 33(42) (2013) 16471-82.

[48] R. Gupta, L. Truong, D. Bear, D. Chafik, E. Modafferi, C.T. Hung, Shear stress alters the expression of myelin-associated glycoprotein (MAG) and myelin basic protein (MBP) in Schwann cells, J Orthop Res 23(5) (2005) 1232-9.

[49] M.C. Liu, V. Akle, W. Zheng, J. Kitlen, B. O'Steen, S.F. Larner, J.R. Dave, F.C. Tortella, R.L. Hayes, K.K. Wang, Extensive degradation of myelin basic protein isoforms by calpain following traumatic brain injury, J Neurochem 98(3) (2006) 700-12.

[50] J.T. Weber, Altered calcium signaling following traumatic brain injury, Front Pharmacol 3(60) (2012) 60.

[51] L.M. Lund, I.G. McQuarrie, Calcium/calmodulin-dependent protein kinase IIbeta isoform is expressed in motor neurons during axon outgrowth and is part of slow axonal transport, J Neurosci Res 67(6) (2002) 720-8.

[52] M.M. Folkerts, E.A. Parks, J.R. Dedman, M.A. Kaetzel, B.G. Lyeth, R.F. Berman, Phosphorylation of calcium calmodulin-dependent protein kinase II following lateral fluid percussion brain injury in rats, J Neurotrauma 24(4) (2007) 638-50.

[53] M. Zhang, H. Shan, Z. Gu, D. Wang, T. Wang, Z. Wang, L. Tao, Increased expression of calcium/calmodulin-dependent protein kinase type II subunit delta after rat traumatic brain injury, J Mol Neurosci 46(3) (2012) 631-43.

[54] J. Li, X.Y. Li, D.F. Feng, D.C. Pan, Biomarkers associated with diffuse traumatic axonal injury: exploring pathogenesis, early diagnosis, and prognosis, J Trauma 69(6) (2010) 1610-8. 
[55] T.M. Evans, H. Van Remmen, A. Purkar, S. Mahesula, J.A. Gelfond, M. Sabia, W. Qi, A.L. Lin, C.A. Jaramillo, W.E. Haskins, Microwave \& Magnetic (M(2)) Proteomics of a Mouse Model of Mild Traumatic Brain Injury, Transl Proteom 3 (2014) 10-21. [56] B.P. Lucke-Wold, R.C. Turner, A.F. Logsdon, J.E. Bailes, J.D. Huber, C.L. Rosen, Linking traumatic brain injury to chronic traumatic encephalopathy: identification of potential mechanisms leading to neurofibrillary tangle development, J Neurotrauma 31(13) (2014) 1129-38.

[57] R. Mannix, W.P. Meehan, J. Mandeville, P.E. Grant, T. Gray, J. Berglass, J. Zhang, J. Bryant, S. Rezaie, J.Y. Chung, N.V. Peters, C. Lee, L.W. Tien, D.L. Kaplan, M. Feany, M. Whalen, Clinical correlates in an experimental model of repetitive mild brain injury, Ann Neurol 74(1) (2013) 65-75.

[58] W.J. Yang, W. Chen, L. Chen, Y.J. Guo, J.S. Zeng, G.Y. Li, W.S. Tong, Involvement of tau phosphorylation in traumatic brain injury patients, Acta Neurol Scand 135(6) (2017) 622-627.

[59] O. Albayram, A. Kondo, R. Mannix, C. Smith, C.Y. Tsai, C. Li, M.K. Herbert, J. Qiu, M. Monuteaux, J. Driver, S. Yan, W. Gormley, A.M. Puccio, D.O. Okonkwo, B. Lucke-Wold, J. Bailes, W. Meehan, M. Zeidel, K.P. Lu, X.Z. Zhou, Cis P-tau is induced in clinical and preclinical brain injury and contributes to post-injury sequelae, Nat Commun 8(1) (2017) 1000.

[60] R. Rubenstein, B. Chang, J.K. Yue, A. Chiu, E.A. Winkler, A.M. Puccio, R. DiazArrastia, E.L. Yuh, P. Mukherjee, A.B. Valadka, W.A. Gordon, D.O. Okonkwo, P. Davies, S. Agarwal, F. Lin, G. Sarkis, H. Yadikar, Z. Yang, G.T. Manley, K.K.W. Wang, S.R. Cooper, K. Dams-O'Connor, A.J. Borrasso, T. Inoue, A.I.R. Maas, D.K. Menon, 
D.M. Schnyer, M.J. Vassar, Comparing Plasma Phospho Tau, Total Tau, and Phospho Tau-Total Tau Ratio as Acute and Chronic Traumatic Brain Injury Biomarkers, JAMA Neurol 74(9) (2017) 1063-1072.

[61] J.A. Goodrich, J.H. Kim, R. Situ, W. Taylor, T. Westmoreland, F. Du, S. Parks, G. Ling, J.Y. Hwang, A. Rapuano, F.A. Bandak, N.C. de Lanerolle, Neuronal and glial changes in the brain resulting from explosive blast in an experimental model, Acta Neuropathol Commun 4(1) (2016) 124. 


\section{CHAPTER 7 CONCLUSIONS AND FUTURE}

\section{PERSPECTIVES}

Blast-induced mild traumatic brain injury (mTBI) is of particular concern among military personnel due to exposure to blast energy during military training and combat. The impact of primary low-intensity blast (LIB) mediated pathophysiology upon later neurobehavioral disorders has been controversial. Developing a military preclinical blast model to simulate the pathophysiology of human blast injury is an important first step. In this chapter, we provide an overview of primary blast effects and perspectives of our recent studies demonstrating ultrastructural and biochemical changes, including axonal injury, mitochondrial dysfunction, synaptic dysregulation in the brain, and behavioral disorders resulting from open-field blast exposures up to $46.6 \mathrm{kPa}$ using a murine model. The model is scalable and permits exposure to varying magnitudes of primary blast injuries by placing animals at different distances from the blast center or by changing the amount of $\mathrm{C} 4$ charge. We here review the implications and future applications and directions of using this animal model to uncover the underlying mechanisms related to primary blast injury. Overall, these studies offer the prospect of enhanced understanding of the pathogenesis of primary LIB-induced TBI and insights for prevention, diagnosis and treatment of blast induced TBI, particularly $\mathrm{mTBI}$ / concussion related to current combat exposures.

Military personnel in theater of operations or during combat training are frequently exposed to blast waves produced by explosive weaponry, which can cause varying severity of TBIs. Blast-induced mTBI is the most common form of TBI and is 
regarded as a 'signature wound' or 'invisible injury' of current combat activities. During 2000-2017, the Department of Defense reported that the vast majority (>82\%) of TBIs were classified as mTBI/concussion related to blast injury. Blast-induced mTBIs are usually not detectable using conventional imaging techniques including computerized tomographic scanning. They are characterized by scores of $13-15$ on the Glasgow Coma Scale (GCS). Immediate signs and symptoms are usually transient [1], however some of these individuals are at a risk for developing post-traumatic stress disorder (PTSD), mental or physical abnormalities, and lifelong disabilities [2,3]. Later sequelae of these injuries impose immense burdens on affected patients, their families, and society.

While past blast injury experimental studies have helped to gain insights into TBI with a range of moderate- to high-intensity blast explosions [4-6], our understanding of the pathogenesis of primary LIB-induced brain injury and its relation to later neurological outcomes remains poorly characterized. The pathogenesis of LIB injury from the primary blast shockwaves likely differs from that caused by impact/acceleration injuries. Therefore, an understanding of the neuropathological and cellular outcomes and associated behavioral consequences after blast exposures including primary LIB is needed. Additionally, despite intensified research efforts and fortified support, methods that would provide early and reliable insights into the progress of TBI / blast-induced brain damage, or a magic cocktail that would prevent delayed brain damage and / or replace or regenerate damaged cells after injury, have yet to be developed [7]. Our knowledge of the pathobiology of TBI / blast injury (including mechanisms of neuronal cell death, neurodegeneration and inflammation in the brain) is gradually increasing, but 
we are still far from having effective neuroprotective therapies that would help the large number of people who suffer from debilitating effects of TBI.

Experimental animal models have been widely used to address the fundamental questions related to the primary blast injury. Primary LIB injury models ideally should adhere to blast physics encountered in relevant combat scenarios. Detonation of high energy explosives entails almost instantaneous chemical decomposition with abrupt generation of kinetic, light, heat energy, sound, and generation of a supersonic shockwave front and overpressure followed by a blast wave [8]. The primary blast wave is characterized by velocity in excess of the speed of sound in air, peak overpressure, duration, and impulse (integration of overpressure with respect to time). The Friedlander curve describes propagation of an ideal blast wave through time and space. However, this idealized waveform representation is altered by environmental interactions for an openfield blast exposure with a characteristic 'ground bounce' when the explosive and target are above ground [8]. Our recent studies have provided a highly reproducible, open-field primary LIB injury mouse model which can be further scaled up for larger animals [912]. The Missouri Blast model employs an open-field detonation of a calibrated 350 grams of high-energy $\mathrm{C} 4$ explosive to generate low-intensity primary blast waves (a static peak overpressure of $46.6 \mathrm{kPa}$ and a maximal impulse of $8.7 \mathrm{PSI} \times \mathrm{ms}$ ) manifesting a Friedlander waveform coupled with an enhanced impulse due to the 'ground bounce'. Using this primary LIB injury mouse model, we have investigated potential links between blast physics and biological outcomes.

A prior physical model suggested that exposure(s) to primary blast waves may lead to nanoscale brain injury [13]. This injury paradigm suggested further study of the 
link between the biomedical outcomes and physical mechanisms causing mTBI. Our prior blast physics modeling predicted that the shockwave front traveling through liquid matters in the brain excites a phonon continuum decaying into specific acoustic waves with intensity exceeding the brain tissue's strength [14]. This physical model predicted that energy deposited behind the primary blast wave causes nanoscale tissue damages occurring within microseconds ( $\mu \mathrm{sec}$ ), well before the head acceleration. The model predicted periodic pressure waves at $\sim 200$ nanometer $(\mathrm{nm})$ intervals with rupture peaks at $\sim 4 \mathrm{~nm}$, where peaks of the energy waves are the greatest. Accordingly, primary LIB injury causes nanoscale subcellular damage, biochemical and molecular changes. The initial cellular effects predicted by these model calculations are much smaller and beyond the resolution offered by conventional imaging techniques and light microscopy. In accordance with consideration of blast physics predicting ultrastructural injuries at nanoscale levels, we investigated fine structural neuropathological changes after primary LIB injury using TEM and measured associated behavioral impairments. High speed videography confirmed that the open-field blast waves pass freely through the mice with no apparent head acceleration / rotation or bodily movement under such blast settings [9]. As a result, the following biological consequences were attributable to the primary LIB wave effects. We observed the absence of macroscopic damage/necrosis and no apparent evident astrogliosis at blast exposure levels up to $46.6 \mathrm{kPa}$.

Neuropathologically, myelinated axonal injury using silver staining was identified in blast exposed mice at 7 days post injury (DPI) $(\mathrm{P}<0.0001)$, but not at $30 \mathrm{DPI}(\mathrm{P}>0.05)$, suggesting some degrees of spontaneous recovery of myelinated axons. Importantly, as the blast wave passed through the brain from rostral to caudal, the level of silver staining 
intensities decreased, suggesting progressive reduction of the blast energy unloading occurred with greater damage to frontal brain areas. Using TEM, we observed and quantified myelin sheath defects at 7 DPI $(\mathrm{P}<0.001)$, but not 30 DPI $(\mathrm{P}>0.05)$, consistent with the silver staining findings. However, in contrast to the silver staining and myelination findings in axons, we observed and quantified persistent mitochondrial abnormalities both at 7 and 30 DPI $(\mathrm{P}<0.0001)$. We observed extensive split layers, dense degeneration, ballooning, and disruption of the myelin in corpus callosum. We also noted swollen clear / dense and degenerated mitochondria in cortex, hippocampus, and striatum after LIB exposure. In addition, we further identified multifocal neuronal damages and synaptic dysregulation after LIB injury. Specifically, we found dysmorphic dark neuronal perikaryon and "cytoplasmic aeration" of dendritic processes, as well as increased microtubular fragmentation of the myelinated axons along with biochemically measured elevated tau / phosphorylated tau / A $\beta$ levels. The number of cortical excitatory synapses decreased with a compensatory increase of the post-synaptic density (PSD) thickness both at 7 and 30 DPI, while the amount of hippocampal CA1 synapses increased with the reduced PSD thickness. Additionally, we observed a significant increase in protein levels of PSD95 and synaptophysin mainly at 7 DPI indicating potential synaptic reorganization. Our study further demonstrated transient neurobehavioral dysfunctions including: decreased locomotor activity, increased anxiety-like behaviors, compromised nesting behavior, and mild spatial learning and memory deficits after primary LIB injury. As we observed such morphological changes after the blast injury, next, we investigated the in-depth underlying mechanisms related to axonal injury and mitochondrial dysfunction assisted by quantitative proteomics approach and biochemical 
studies. First, we used tandem mass tagged (TMT) quantitative proteomics and bioinformatics analysis to seek insights into the molecular mechanisms underlying ultrastructural pathology. We found striking dynamic changes in both global and phosphorylated proteins at various time points after blast. Disruption of key canonical pathways included evidence of mitochondrial dysfunction, oxidative stress, axonal/cytoskeletal/synaptic dysregulation, and neurodegeneration. Bioinformatic analysis identified blast induced trends in networks related to cellular growth / development / movement / assembly and cell-to-cell signaling interactions. With observations of proteomic changes, we found LIB-induced oxidative stress associated with mitochondrial dysfunction mainly at 7 and 30 DPI. These dysfunctions included impaired fission-fusion dynamics, diminished mitophagy, decreased oxidative phosphorylation, and compensated respiration-relevant enzyme activities. Second, we also identified alterations of axonal and synaptic proteins in related pathways, including but not being limited to substantia nigra degeneration, cortical cytoskeleton organization and synaptic vesicle exocytosis, suggesting a potential axonal damage caused by blastinduced mTBI. We also found blast induced elevated phospho-tau, a pathological feature found in brains of $\mathrm{AD}$, and altered a number of neurophysiological processes, supporting the notion that blast-induced mTBI as a risk factor contributes to AD pathogenesis. Overall, LC/MS-based profiling has presented candidate target / pathways that could be explored for future therapeutic development.

As about $15 \%$ of mTBI cases chronically associate with PTSD and other cognitive dysfunctions, it is often difficult to differentiate between the effects of blast mTBI and PTSD, which may also be the results of psychological stress / emotional 
trauma [15-17]. In order to address this issue in our current animal model, future directions will include animal re-exposure to the testing ground to determine whether the observed behavioral changes might be re-introduced by the blast context in absence of actual blast exposures. Our current studies have uncovered unique ultrastructural brain abnormalities at nanoscale levels and associated neurobehavioral impairments due to primary blast injury. These findings address the critical gaps in knowledge about mTBI and provide insights into its pathogenesis of mTBI.

Our novel findings suggest the progression of additional molecular changes leading to secondary injuries, including mitochondrial dysfunction and disruption of axonal transport. Recent evidence suggests that mitochondria play a critical role in TBI, as impairments of mitochondrial bioenergetics may be linked to neuronal excitotoxicity, disruption of $\mathrm{Ca}^{2+}$ homeostasis, production of reactive oxygen species (ROS), neuroinflammation, and ATP depletion [18]. Further, the mitochondrial changes may lead to release of pro-apoptotic proteins causing potential neuronal damage. Defects in mitochondrial bioenergetics and associated metabolic function are also known to be linked with alterations of the maintenance of mitochondrial integrity, including mitochondrial clearance/dynamics. These effects can in turn, impact axonal transport and autophagy [19]. Although mitochondrial dysfunction and bioenergetic failure have been implicated in TBI and in certain neurodegenerative diseases, it remains unclear whether such mitochondrial dysfunction is a cause or an effect of the underlying pathology. And whether or not mitochondrial dysfunction represents a viable therapeutic target particularly in primary blast injury to prevent or ameliorate its long term effects [20, 21]. Future investigations will require a focus upon direct links between primary LIB-induced 
changes in mitochondrial structures and functions and resulting cellular damages or neurochemical imbalance, including respiration, DNA/mRNA levels, and key proteins influencing energy production that may ultimately affect long-term brain functions.

In summary, preclinical animal models of TBI have provided substantial information about primary blast injury, but gaps remain in translating the pathophysiology of animal injury to human injury [22]. Ideally, autopsy studies of the human brain exposed to explosive devices are important and should be used to elucidate late injury effects. However, changing neurological and behavioral abnormalities observed in patients with TBI or blast injury may vary depending upon subtle of cellular, sub-cellular and molecular pathological processes. To study such changes, it remains important to use animal models that best simulate actual conditions of blast exposure, though differences in properties of soft tissues of the head or the major anatomical distinctions between human and animals remain as a limitation to be taken into account $[23,24]$; sensitive and respectful use of non-human primates suggests way of overcoming this limitation. Accurate and reproducible data are critical in translating and scaling animal findings into human injuries. Biological models using well defined blast conditions, detailed characterization of the physics of exposure, and model standardization offer better alignment between the actual blast exposures and biological consequences seen in real-life scenarios involving human subjects. As recently reported, we have implemented monitoring systems that ensure consistency and quality control of blast exposure in our open-field model. Our findings show that a primary LIB injury appears to contribute "invisible injuries", solely detectable at ultrastructural levels and associated with well-defined neurobehavioral dysfunctions. The Missouri Blast model, 
provides a means to define underlying mechanisms of primary blast injury, the effects of repetitive blast exposures, sex differences, and body positions relative to blast source and intensity. Such quantitative observations will provide insights for preventing blast injuries and treatment blast-induced brain trauma. While further investigation is required, the resulting neuropathological and behavioral changes seen in this primary blast model appear to provide a link between experimental injury and human $\mathrm{mTBI} /$ concussion effects. Further, we are currently considering use of telemetric sensor probes to explore blast intensity and intracranial pressure effects.

The past decade of blast injury research yielded significant and novel findings regarding the blast physics and biological effects. Our recent observations revealed that primary LIB injury comprises an 'invisible injury' characterized by the nanoscale ultrastructural injuries, mitochondrial and axonal biochemical alterations, and accompanied by neurobehavioral dysfunction up to 30 days after exposure. Using the Missouri Blast model, we hope to further link blast physics to underlying injury mechanisms occurring as animals related to various blast conditions including repetitive injury as well as in late non acute stages after blast injury. Further translational studies will advance in development of targeted treatment and prevention to blast-associated delayed cognitive dysfunctions manifested in chronic neurodegenerative disorders. 


\subsection{REFERENCES}

[1] S.T. DeKosky, M.D. Ikonomovic, S. Gandy, Traumatic Brain Injury -- Football, Warfare, and Long-Term Effects., New England Journal of Medicine 363(14) (2010) 1293-1296.

[2] R.G. DePalma, D.G. Burris, H.R. Champion, M.J. Hodgson, Blast injuries, New England Journal of Medicine 352(13) (2005) 1335-42.

[3] G.S. Griesbach, B.E. Masel, R.E. Helvie, M.J. Ashley, The Impact of Traumatic Brain Injury on Later Life: Effects on Normal Aging and Neurodegenerative Diseases, Journal of Neurotrauma 35(1) (2018) 17-24.

[4] I. Cernak, A.C. Merkle, V.E. Koliatsos, J.M. Bilik, Q.T. Luong, T.M. Mahota, L. Xu, N. Slack, D. Windle, F.A. Ahmed, The pathobiology of blast injuries and blast-induced neurotrauma as identified using a new experimental model of injury in mice, Neurobiology of Disease 41(2) (2011) 538-551.

[5] M.D. Budde, A. Shah, M. McCrea, W.E. Cullinan, F. Pintar, B.D. Stemper, Primary blast traumatic brain injury in the rat: relating diffusion tensor imaging and behavior, Frontiers in Neurology 4 (2013) 154.

[6] H. Wang, Y.P. Zhang, J. Cai, L.B. Shields, C.A. Tuchek, R. Shi, J. Li, C.B. Shields, X.-M. Xu, A compact blast-induced traumatic brain injury model in mice, Journal of Neuropathology \& Experimental Neurology 75(2) (2016) 183-196.

[7] I. Cernak, D.G. Stein, G.A. Elder, S. Ahlers, K. Curley, R.G. DePalma, J. Duda, M. Ikonomovic, G.L. Iverson, F. Kobeissy, V.E. Koliatsos, M.J. Leggieri, A.M. Pacifico, D.H. Smith, R. Swanson, F.J. Thompson, F.C. Tortella, Preclinical modelling of 
militarily relevant traumatic brain injuries: Challenges and recommendations for future directions, Brain Injury 31(9) (2017) 1168-1176.

[8] M.D. Goel, V.A. Matsagar, A.K. Gupta, S. Marburg, An Abridged Review of Blast Wave Parameters, Defence Science Journal 62(5) (2012) 300-306.

[9] H. Song, L.M. Konan, J. Cui, C.E. Johnson, M. Langenderfer, D. Grant, T. Ndam, A. Simonyi, T. White, U. Demirci, D.R. Mott, D. Schwer, G.K. Hubler, I. Cernak, R.G. DePalma, Z. Gu, Ultrastructural brain abnormalities and associated behavioral changes in mice after low-intensity blast exposure, Behav Brain Res 347 (2018) 148-157.

[10] M. Chen, H. Song, J. Cui, C.E. Johnson, G.K. Hubler, R.G. DePalma, Z. Gu, W. Xia, Proteomic Profiling of Mouse Brains Exposed to Blast-Induced Mild Traumatic Brain Injury Reveals Changes in Axonal Proteins and Phosphorylated Tau, Journal of Alzheimer's Disease (Preprint) (2018) 1-23.

[11] H. Song, L.M. Konan, J. Cui, C.E. Johnson, G.K. Hubler, R.G. DePalma, Z. Gu, Nanometer ultrastructural brain damage following low intensity primary blast wave exposure, Neural Regen Res 13(9) (2018) 1516-1519.

[12] H. Song, J. Cui, A. Simonyi, C.E. Johnson, G.K. Hubler, R.G. DePalma, Z. Gu, Linking blast physics to biological outcomes in mild traumatic brain injury: Narrative review and preliminary report of an open-field blast model, Behav Brain Res 340 (2018) $147-158$.

[13] Y. Kucherov, G.K. Hubler, R.G. DePalma, Blast induced mild traumatic brain injury/concussion: A physical analysis, Journal of Applied Physics 112(10) (2012) 104701. 
[14] Y. Kucherov, G. Hubler, J. Michopoulos, B. Johnson, Acoustic waves excited by phonon decay govern the fracture of brittle materials, Journal of Applied Physics 111(2) (2012) 023514.

[15] C.L. Mac Donald, A.M. Johnson, D. Cooper, E.C. Nelson, N.J. Werner, J.S.

Shimony, A.Z. Snyder, M.E. Raichle, J.R. Witherow, R. Fang, Detection of blast-related traumatic brain injury in US military personnel, New England Journal of Medicine 364(22) (2011) 2091-2100.

[16] B.E. Wisco, B.P. Marx, D.W. Holowka, J.J. Vasterling, S.C. Han, M.S. Chen, J.L. Gradus, M.K. Nock, R.C. Rosen, T.M. Keane, Traumatic brain injury, PTSD, and current suicidal ideation among Iraq and Afghanistan US veterans, Journal of Traumatic Stress 27(2) (2014) 244-248.

[17] C.L. Mac Donald, J. Barber, M. Jordan, A.M. Johnson, S. Dikmen, J.R. Fann, N. Temkin, Early Clinical Predictors of 5-Year Outcome After Concussive Blast Traumatic Brain Injury, JAMA Neurology 74(7) (2017) 821-829.

[18] J.B. Hiebert, Q. Shen, A.R. Thimmesch, J.D. Pierce, Traumatic brain injury and mitochondrial dysfunction, The American Journal of the Medical Sciences 350(2) (2015) $132-8$

[19] T. Misgeld, T.L. Schwarz, Mitostasis in Neurons: Maintaining Mitochondria in an Extended Cellular Architecture, Neuron 96(3) (2017) 651-666.

[20] E. Lezi, R.H. Swerdlow, Mitochondria in neurodegeneration, Advances in Experimental Medicine and Biology 942 (2012) 269-86.

[21] L.T. Watts, Stimulating mitochondria to protect the brain following traumatic brain injury, Neural Regeneration Research 11(9) (2016) 1403-1404. 
[22] I. Cernak, D.G. Stein, G.A. Elder, S. Ahlers, K. Curley, R.G. DePalma, J. Duda, M. Ikonomovic, G.L. Iverson, F. Kobeissy, Preclinical modelling of militarily relevant traumatic brain injuries: Challenges and recommendations for future directions, Brain Injury 31(9) (2017) 1168-1176.

[23] A. Jean, M.K. Nyein, J.Q. Zheng, D.F. Moore, J.D. Joannopoulos, R. Radovitzky, An animal-to-human scaling law for blast-induced traumatic brain injury risk assessment, Proceedings of the National Academy of Sciences 111(43) (2014) 15310-15315.

[24] Y. Xiong, A. Mahmood, M. Chopp, Animal models of traumatic brain injury, Nature Review Neuroscience 14(2) (2013) 128-42. 


\section{VITA}

Hailong Song was born on April 1st, 1990, in Tianjin, China. After graduating from Tianjin Experimental High School in 2008, Mr. Hailong Song was admitted by Capital Medical University (CMU), where he achieved a degree of Bachelor of Medicine (BM) in department of Basic Medical Sciences in June 2013. In 2014, Hailong Song began his Ph.D. graduate studies and worked for his dissertation under the mentorship of Dr. Zezong Gu at the Department of Pathology and Anatomical Sciences in the University of Missouri-Columbia. This thesis is in compliance with his completion of a Doctor of Philosophy (Ph.D.) in Interdisciplinary Neuroscience Program. After his Ph.D. study, he will continue pursuing his passionate career as a neuroscientist. 